\title{
Functional Properties of Hybrid Resonances in Plasmonic Nanocrystals
}

\author{
by
}

Daniel Prezgot

A thesis submitted to the Faculty of Graduate and Postdoctoral Affairs in partial fulfillment of the requirements for the degree of

Doctor of Philosophy

in

Chemistry

Carleton University

Ottawa, Ontario

(C) 2020, Daniel Prezgot 


\section{Abstract}

Metal nanocrystals support unique light-matter interactions through a phenomenon known as the localized surface plasmon resonance (LSPR). These resonances show a great deal of sensitivity to their local environment, and by altering this environment it is possible to produce new, "hybridized" plasmon modes. The far-field spectral qualities, and the near-field electromagnetic properties can by heavily manipulated through the generation of hybridized plasmon modes. This work explores the properties and functions of hybridized plasmon modes in a variety of systems. Finite-difference time-domain modelling use used to correlate the experimental spectral response is with the calculated near-field spatial distribution of hybridized modes in a number of systems involving silver nanocubes (AgNC). These systems demonstrate it is possible to carefully monitor the environment of a nanocrystal, induce an unusually sharp spectral extinction in dielectric-coated AgNCs, and manipulate the spatial distribution of plasmon modes in colloidal composite $\mathrm{Ag} @ \mathrm{Cu}_{2} \mathrm{O}$ core-shell nanocrystals. These properties are applied to functional materials, including photothermal and colour patterning of a plasmonic system driven by the embedment of an AgNC into a polymer matrix. The spectral signature of hybridized plasmon modes is used to both characterize and manipulate the degree of photothermal heating in this thermoplasmonic patterning system. Hybridized gap-plasmons are used to generate wide range of colours, with controllable palettes using AgNC-over-Au and AgNC-over-Ag nanoparticle-over-mirror (NPoM) films. Lastly the potential for alternative plasmonic materials for ultraviolet (UV) plasmonics was explored. The potential for In and Al nanocrystals for UV surface-enhanced Raman spectroscopy was investigated. All these systems demonstrate the potential and advantage of using hybridized plasmon modes to manipulate the far-field and near-field optical response of functional plasmonic materials. 


\section{Preface}

This preface provides full bibliographical details for each article included in this thesis, as well as whether the article is reproduced in whole or in part. Use of copyrighted material is likewise acknowledged here. When citing material from this thesis, please cite the article relevant to the chapter, if the chapter is based on a publication.

Pursuant to the Integrated Thesis policy of Carleton University, the "supervisor" (Anatoli lanoul) and the student (Daniel Prezgot) confirm that the student was fully involved in setting up and conducting the research, obtaining data and analyzing results, as well as preparing and writing the material presented in the co-authored article(s) integrated in the thesis. Additionally, the supervisor confirms the information provided by the student in this preface.

\section{Chapter 1}

Figure 1.1 was adapted from Daniel Prezgot, @ 2014

Figures 1.3 a,b were reproduced under the open access Creative Commons license 4.0.

Figure 1.7 was reproduced with permission from Springer Nature, 2015

Figure 1.8 was reproduced with permission from the American Chemical Society, 2014

Figure 1.9 was reproduced with permission from John Wiley and Sons, 2009

Figure 1.10 was reproduced with permission from American Chemical Society, 2011

Figure 1.11 was adapted from Daniel Prezgot, @ 2014

\section{Chapter 2}

This chapter contains three parts. In all cases Daniel Prezgot was primarily responsible for numerical calculations, and in some cases contributed to the experimental work. The experimental work was primarily conducted by the other authors. The sections in this thesis have been edited to focus on the numerical modelling work performed by Daniel Prezgot. Specific contributions to the sub-chapters are as follows:

A) Bottomley, A.; Prezgot, D.; Coyle, J. P.; lanoul, A. Dynamics of Nanocubes Embedding into Polymer Films Investigated via Spatially Resolved Plasmon Modes. Nanoscale 2016, 8 (21), 11168-11176.

This article is partially reproduced with permission.

Daniel Prezgot performed numerical modelling calculations and collaborated on the interpretation of results. Experimental work was primarily performed by Adam Bottomley and Jason P. Coyle. Writing of the original article collaborative between Adam Bottomley, Daniel Prezgot, and Anatoli lanoul.

The article has been trimmed and edited from its original format to focus on work performed by Daniel Prezgot. Other sections of the original work remain in order to provide context. Most of the content included in this thesis was written by Daniel Prezgot 
B) Prezgot, D.; Bottomley, A.; Coyle, J. P.; lanoul, A. Unusually Sharp Localized Surface Plasmon Resonance in Supported Silver Nanocrystals with a Thin Dielectric Coating. J. Phys. Chem. Lett. 2017, 8 (22), 5555-5558.

This article is wholly reproduced with permission, with some editing for format and clarity.

The student performed numerical modelling calculations, surface-enhanced Raman spectroscopy measurements and the interpretation of results. Fabrication and characterization of the samples was primarily performed by Adam Bottomley and Jason P. Coyle. The original article was written collaboratively by all co-authors, primarily by Anatoli lanoul.

C) Gale-Mouldey, A.; Jorgenson, E.; Coyle J. P.; Prezgot, D.; lanoul, A.; Hybridized plasmon resonances in core/half-shell cuprous oxide nanoparticles., J. Mater Chem. C., 2020, Advance Article

This section is partially reproduced from a manuscript which has been submitted for publication.

Daniel Prezgot performed numerical modelling calculations. Synthesis and characterization of the particles was performed by Alexandra Gale-Mouldey Emma Jorgenson and Jason P. Coyle. Writing of the original manuscript was collaborative between all co-authors, written primarily by Alexandra Gale-Mouldey, and Anatoli lanoul.

The manuscript has been edited from its original format to focus on the modelling work performed by Daniel Prezgot, though some of the experimental results are provided for context. Most of the content included in this thesis was written by Daniel Prezgot.

\section{Chapter 3}

This chapter reproduced from a manuscript which has been submitted for publication:

Prezgot, D.; Bottomley, A.; Jorgenson, E.; lanoul, A. Thermoplasmonic patterning of silver nanocrystal/polymer composite thin films, Submitted for Publication, 2019

Daniel Prezgot performed the majority of the experimental work, calculations and interpretation of results. The project began collaboratively between Daniel Prezgot and Adam Bottomley, Emma Jorgenson produced the AgNC monolayers used in the work. Writing of the original article collaborative between Daniel Prezgot, Adam Bottomley, and Anatoli lanoul, primarily written by Daniel Prezgot.

\section{Chapter 4}

This chapter represents a manuscript which is in the process of being prepared for submission.

Prezgot, D.; Tatarchuk, S.W.; Ianoul, A. Plasmonic Colour Generation in Silver Nanocrystal-overMirror Films by Thermal Embedment into a Polymer Spacer, Manuscript in Preparation

Daniel Prezgot was responsible for the conception of the project, planning of the experimental work, performing the experimental work relating Ag films, and interpretation of results. Stephen Tatarchuk performed the experimental work relating to Au films under the direction of Daniel Prezgot. Numerical calculations were performed by Stephen Tatarchuk and Daniel Prezgot. MATLAB code for data processing was originally written by Stephen Tatarchuk. Writing of the original article was collaborative between all authors, the content included in this thesis was written by Daniel Prezgot. 


\section{Chapter 5}

This chapter represents work which, chronologically, was performed first, the experimental work took place before that of the other chapters. The results were not considered suitable for publication; however, the work was still considered relative to this thesis.

Daniel Prezgot was responsible for the experimental work, analysis, and interpretation of the results. Jason P. Coyle assisted with the synthesis of aluminium nanocrystals. The content included in this thesis was written by Daniel Prezgot. 


\section{Acknowledgements}

Without the generous contribution and support of many individuals, the completion of this work would not have been possible.

First and foremost, I would like to thank Dr. Anatoli lanoul, whose mentorship was invaluable. He kept me along for many years despite the stress and frustration I caused him, continuously encouraged to become a better scientist, to avoid settling for mediocrity (as tempted as I can be to do so), and for believing in me even through times when I did not believe in myself. A kinder and more generous mentor I could not imagine, he has sent me around the world and still encourages me to expand my horizons.

I would also like to thank the revolving door of students and researchers I have worked alongside with in the lanoul group: Adam Bottomley, Devin O'Neill, Jason Coyle, Michael Bushell, Emma Jorgenson, Alexandra Gale-Mouldey, Stephen Tatarchuk and Devron Colley and the other various undergrads who have cycled through the group who either directly or indirectly contributed to my work, and also provided welcome distractions and support along the way. I would also like to thank other students of the Chemistry department, including (but not limited to) Grace Daly and Gilian Thomas, for keeping me sane(-ish) for their constant support and encouragement, and the impromptu trips to Mike's Place. I would also like to thank the rest of the Chemistry Department, for being such an upstanding community, and Chantelle Gravelle for ensuring that I was able to graduate.

I would thank various people for their technical support, such as Dr. Jianqun Wang for his excellent electron microscopy images, Jim Logan for helping us keep our instruments alive, Rob Vandusen, Angela Williams and Peter Gordon with their assistance with thin film fabrication.

And I would also like to thank my parents, Marek and Zofia Prezgot, for their support, encouragement, and for enabling me to dip from the bank of Mom and Dad when I would mismanage my finances. 


\section{Table of Contents}

Abstract.............................................................................................................................. ii

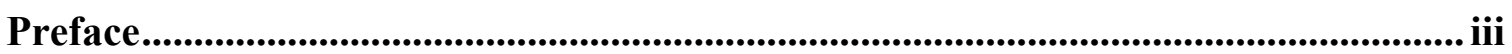

Acknowledgements ........................................................................................................... vi

Table of Contents ..................................................................................................... vii

List of Figures.......................................................................................................................... xii

List of Appendices.......................................................................................................... xiv

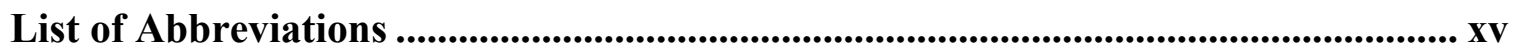

Chapter 1: Introduction ............................................................................................................ 1

$1.1 \quad$ Fundamentals of Plasmonics …………………………………………………...2

1.1.1 Materials for Plasmonics ...............................................................................

$1.2 \quad$ Modelling of Plasmonic Systems ............................................................................

1.2.1 Theoretical basis of modelling methods.............................................................

1.2.2 Finite-difference time-domain calculations ...................................................... 6

$1.3 \quad$ Tuning and Anisotropy in Plasmonic Systems .......................................................... 8

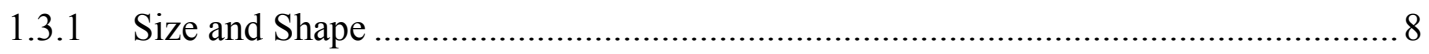

1.3.2 Local environment ............................................................................................

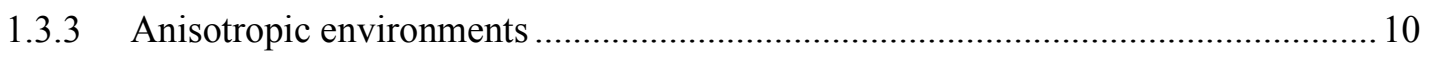

1.3.4 Plasmon Coupling ........................................................................................... 11

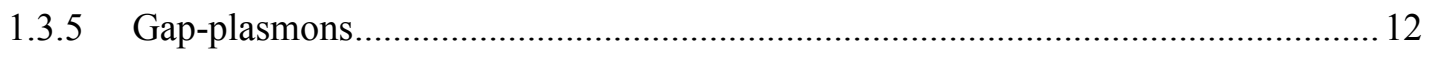

$1.4 \quad$ Applications of Hybrid Plasmon Modes .................................................................. 13

1.4.1 Surface-Enhanced Raman Spectroscopy ……………………………………….... 13

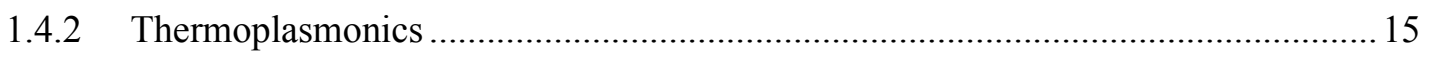

1.4.3 Plasmon Colour Generation .............................................................................. 16

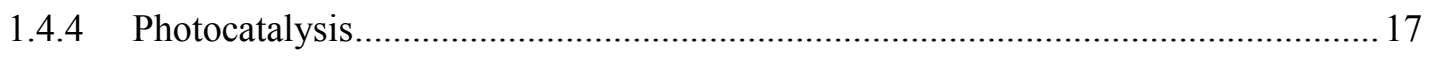




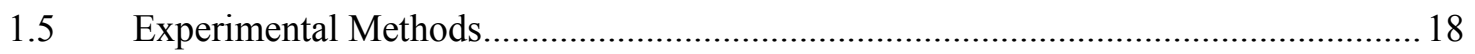

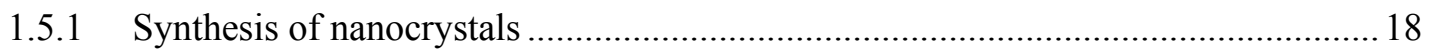

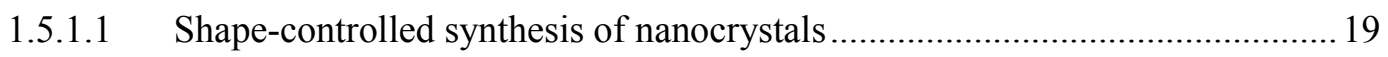

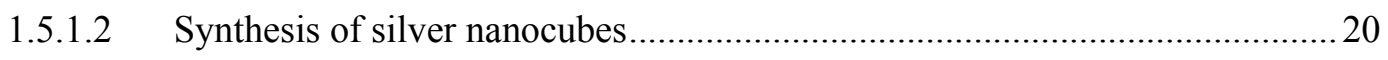

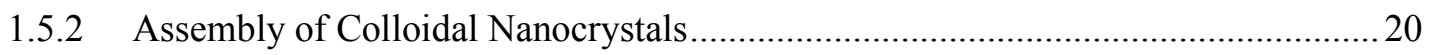

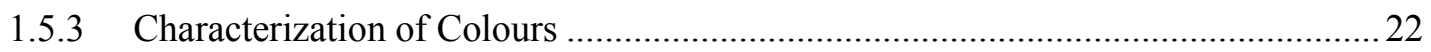

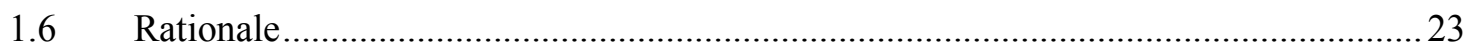

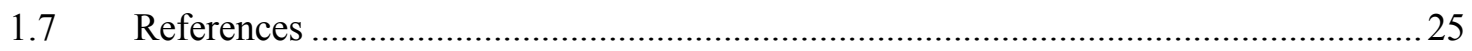

\section{Chapter 2: Numerical Modelling of Hybrid Plasmonic Modes in Anisotropic}

Systems......................................................................................................................... 33

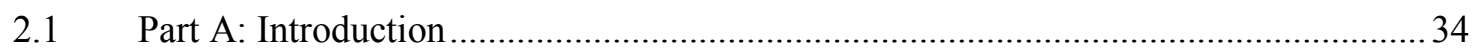

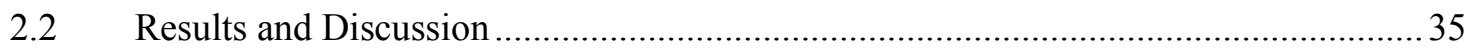

2.2.1 Spectral Characteristics of Embedding Nanocubes into polystyrene........................35

2.2.2 Modelling extinction of partially embedded cubes .............................................. 39

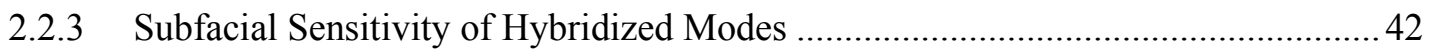

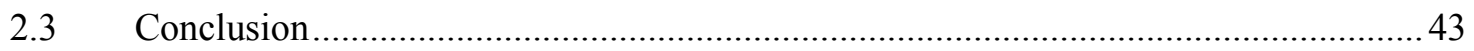

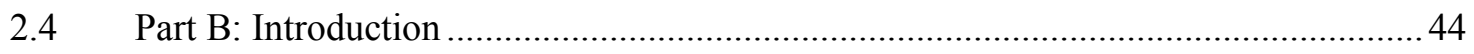

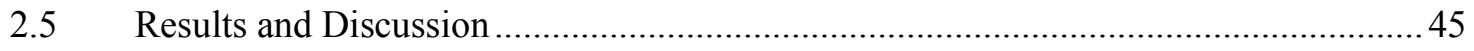

2.5.1 Observation of sharp localized surface plasmon resonances ................................. 45

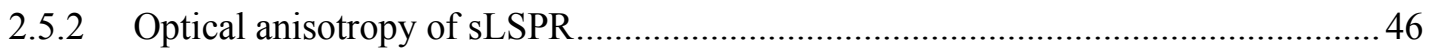

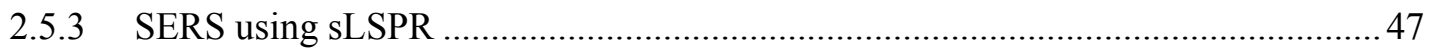

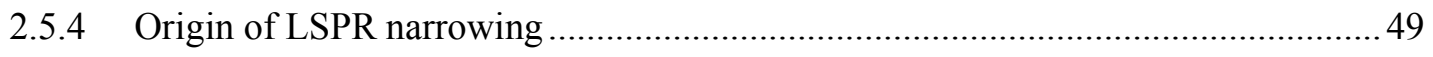

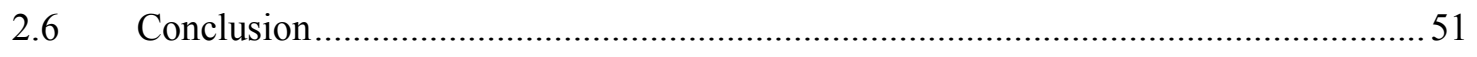

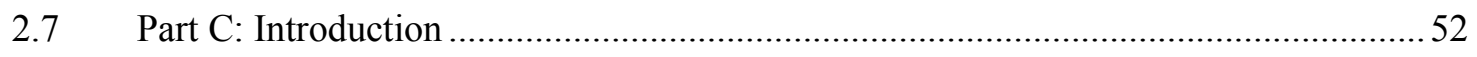

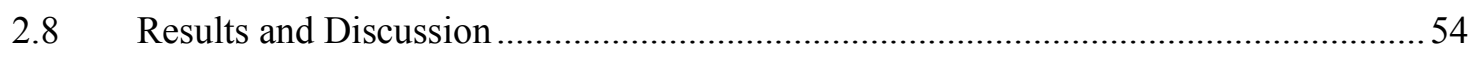

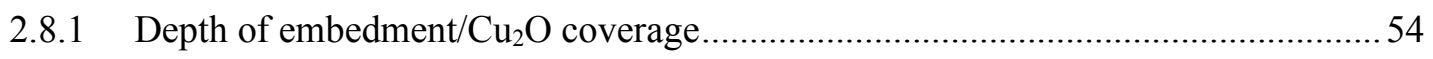




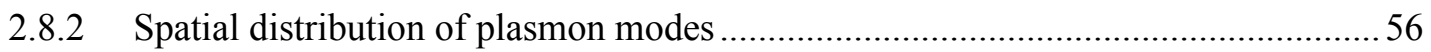

2.8.3 Optimization of geometric parameters and description of peak shifts ....................58

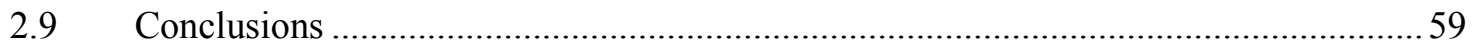

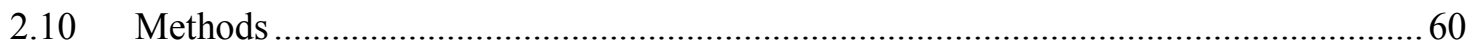

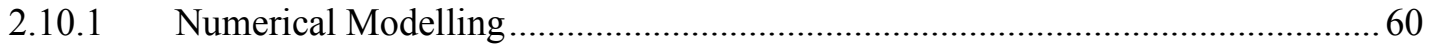

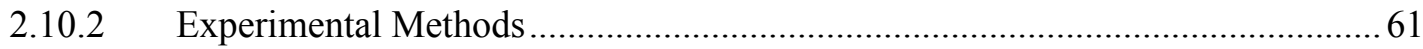

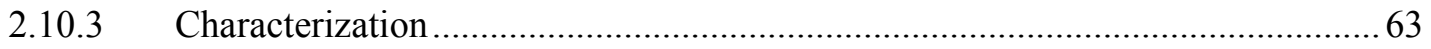

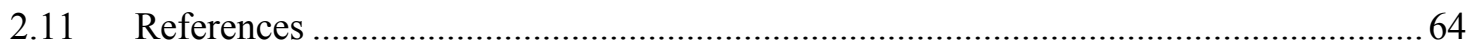

\section{Chapter 3: Thermoplasmonic patterning of silver nanocrystal/polymer composite} thin films ................................................................................................................6 69

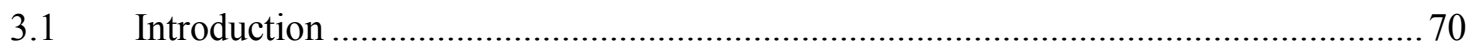

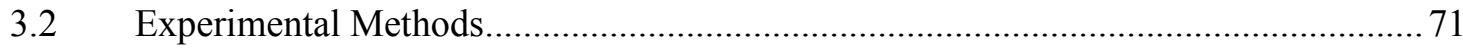

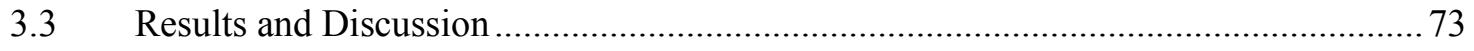

3.3.1 Thermoplasmonic patterning of silver nanocrystal-on-polymer films .................... 73

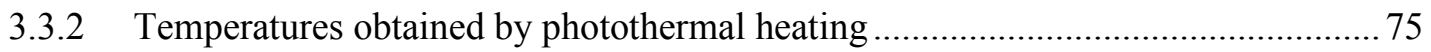

3.3.3 Experimental verification of Temperature ............................................................ 77

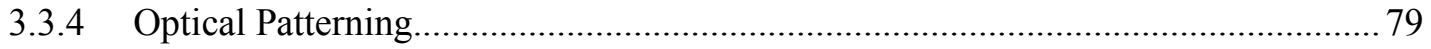

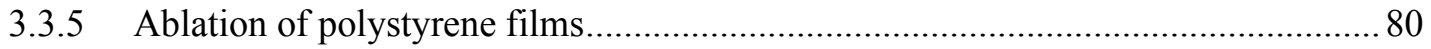

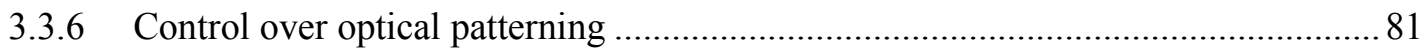

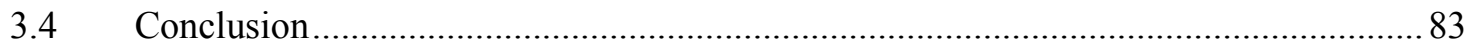

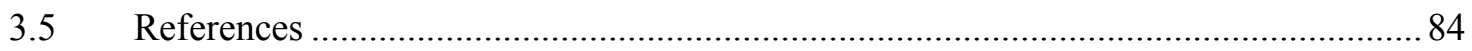

\section{Chapter 4: Plasmonic Colour Generation in Silver Nanocrystal-over-Mirror Films} by Thermal Embedment into a Polymer Spacer..................................................... 87

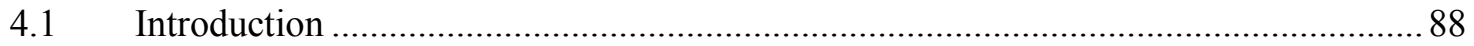

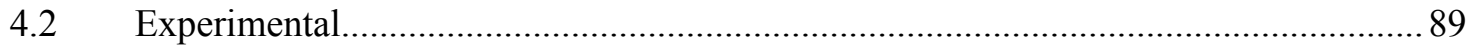

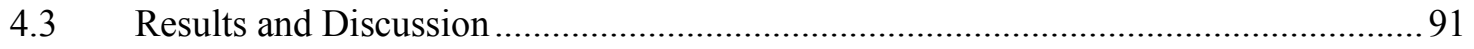


4.3.1 Design of thermally-tunable NPoM composites ................................................ 91

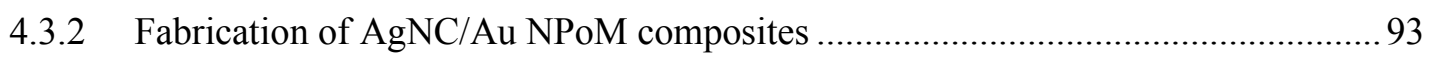

4.3.3 Plasmonic colour generation in AgNC / Au NPoM films ..................................... 93

4.3.4 Plasmonic colour generation in AgNC / Ag NPoM films .................................... 95

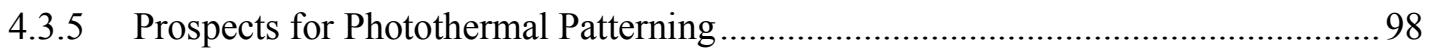

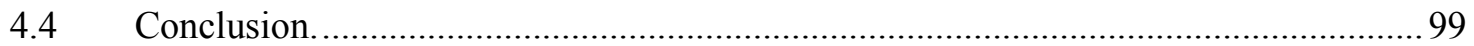

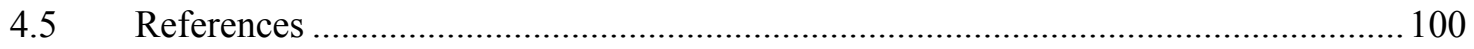

\section{Chapter 5: UV plasmonic materials: Investigating Indium and Aluminium} Nanocrystals for Deep-UV Surface-Enhanced Raman Spectroscopy ....................... 103

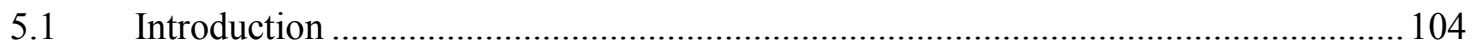

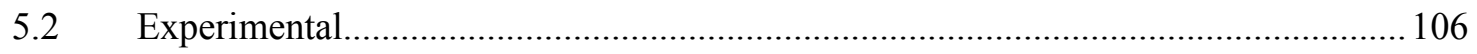

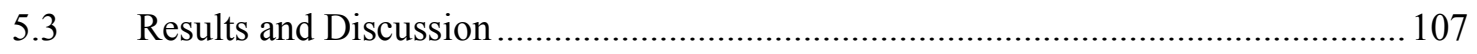

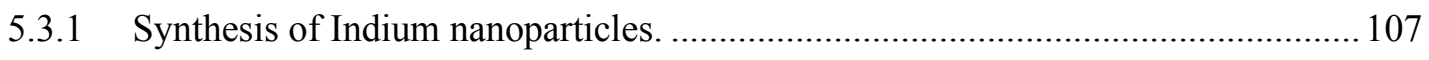

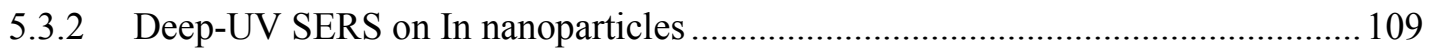

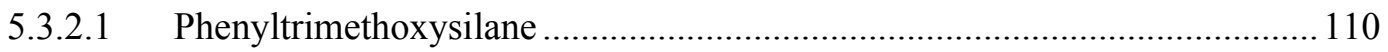

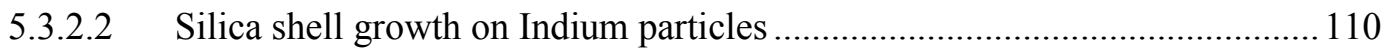

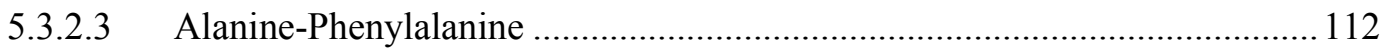

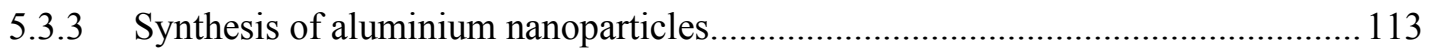

5.3.4 UV Raman spectroscopy over aluminium nanocrystals..................................... 114

5.3.5 Numerical modelling of indium and aluminium nanoparticles............................ 115

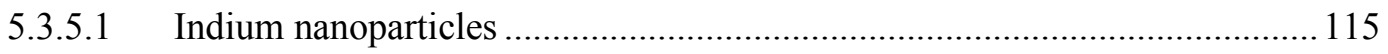

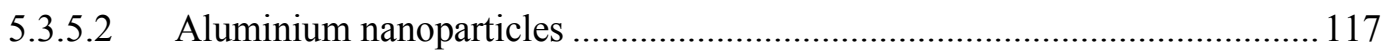

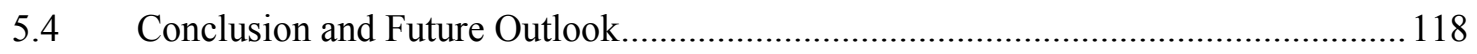

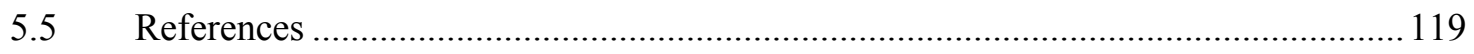

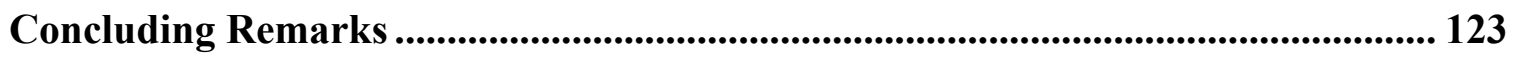


Appendices

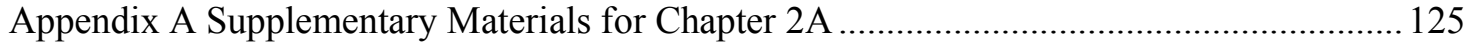

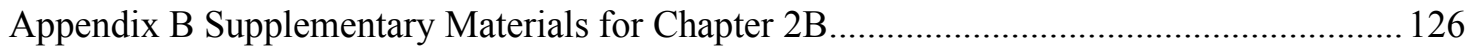

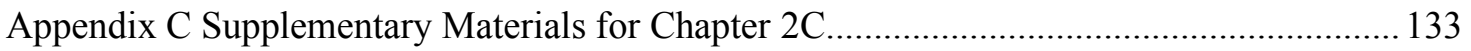

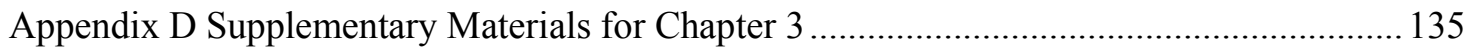

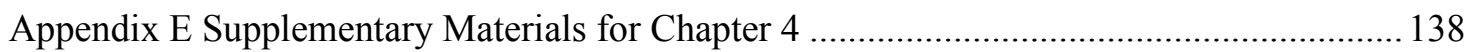

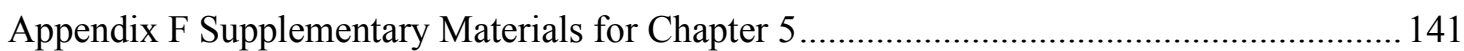

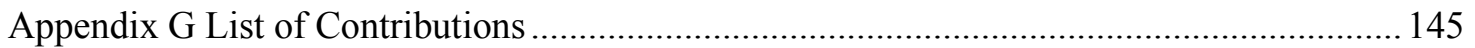




\section{List of Figures}

Figure 1.1. Schematic representation of LSPR ....................................................... 2

Figure 1.2. Dielectric functions of relevant metals................................................... 4

Figure 1.3. Schematic diagram of EM wave and FDTD Yee Cell .................................. 7

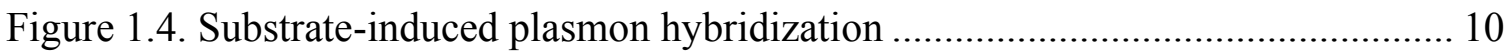

Figure 1.5. Plasmon hybridization in a nanoparticle dimer....................................... 12

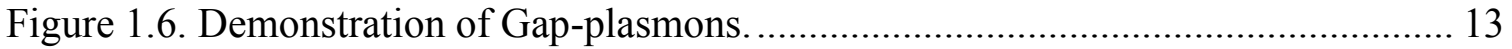

Figure 1.7. Shematic of the lifetime of a plasmon resonance ...................................... 15

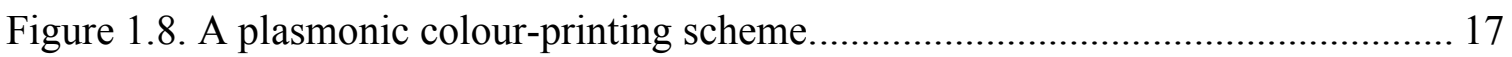

Figure 1.9. LaMer diagram of the growth process of colloidal crystals ......................... 18

Figure 1.10. Shape-controlled synthesis of silver nanostructures................................ 20

Figure 1.11. Langmuir Blodgett Technique................................................................ 21

Figure 1.12. Chromaticity and CIE 1931 Colour Space............................................. 22

Figure 2.1. UV-visible extinction of AgNC on Polystyrene........................................ 36

Figure 2.2 Spectral evolution of the embedment process. ........................................... 38

Figure 2.3 FDTD calculated extinction spectra of a silver nanocube in polystyrene film for different embedding depths and angles ............................................. 40

Figure 2.4 Comparison of peak sensitivity on different $n$ substrates........................... 43

Figure 2.5. Extinction spectra of a silver nanocube monolayer as a function of overlayer

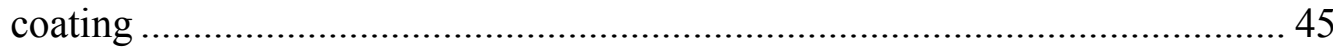

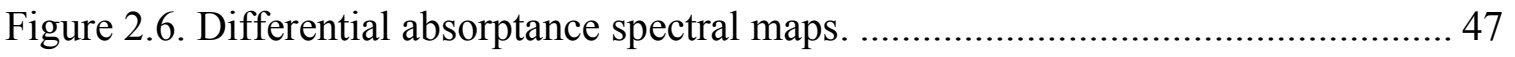

Figure 2.7. SERS excitation profiles for 4-mercaptobenzoic acid............................... 48

Figure 2.8. Calculated density, $\rho$, and electric field intensity, $|\mathrm{E}|^{2}$ for $\mathrm{Al}_{2} \mathrm{O}_{3}$ coated silver

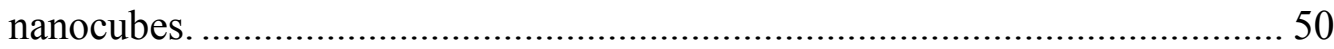

Figure 2.9. Schematic of the core/half-shell synthesis methodology........................... 53

Figure 2.10. TEM images of $\mathrm{Ag} @ \mathrm{Cu}_{2} \mathrm{O}$ half shells, as well as experimental and modelled

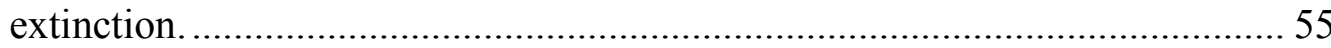

Figure 2.11. 3D view of the charge density, $\rho$, and electric field enhancement, $\left|\mathrm{E} / \mathrm{E}_{0}\right|^{2}$, of plasmonic modes in a $\mathrm{Ag} @ \mathrm{Cu}_{2} \mathrm{O}$ half-shell............................................... 56

Figure 2.12. $\mathrm{Cu}_{2} \mathrm{O}$ half-shell growth of controlled sizes. ....................................... 57 
Figure 2.13. Modelled extinction and electric field of $\mathrm{Cu}_{2} \mathrm{O}$ truncated pyramid half-shells in solution and on polystyrene as they are first synthesized........................ 58

Figure 3.1. Sample characterization for thermoplasmonic patterning, minimum power thresholds for patterning, and photograph of the patterning ........................ 73

Figure 3.2. Summary of three observed states in thermoplasmonic patterning............... 75

Figure 3.3 Stokes/anti-Stokes surface-enhanced Raman spectroscopy of Rh6G on AgNC monolayers with temperature calibration............................................... 78

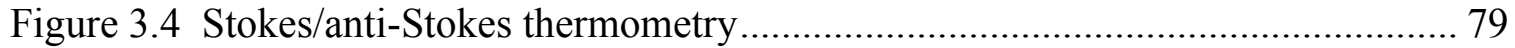

Figure 3.5. Transmittance spectra of a section of the patterned film............................. 80

Figure 3.6. The threshold intensity as a function of absorbance, showing control over the

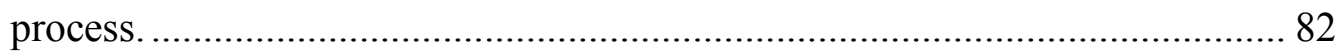

Figure 4.1. Schematic of the NPoM system and model of the plasmon-derived colour

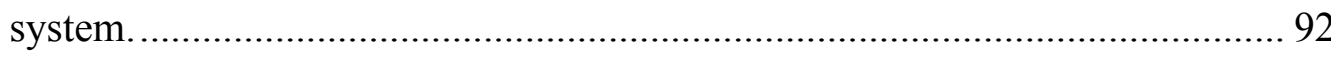

Figure 4.2. Schematic of NPoM fabrication, spectral, and topographic results of

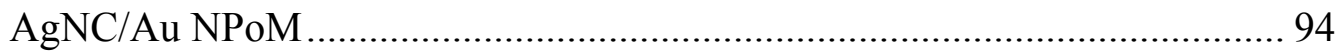

Figure 4.3 Reflectance spectral colormap and results in CIE colourspace.................... 95

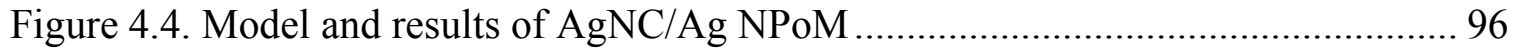

Figure 4.5. AFM topography measurements of the Ag / AgNC sample. ........................ 97

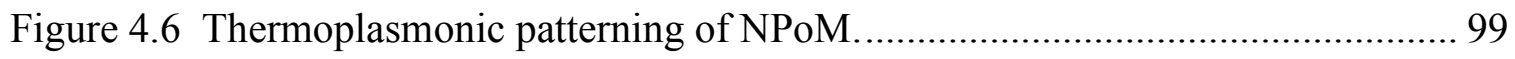

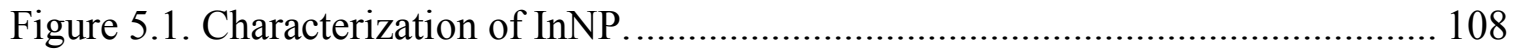

Figure 5.2. Reference UV-Raman spectra of PhTMS and Ala-Phe. ........................... 109

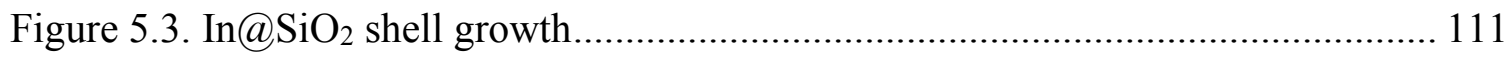

Figure 5.4. Reference UV-Raman spectra of PhTMs and Ala-Phe .............................. 113

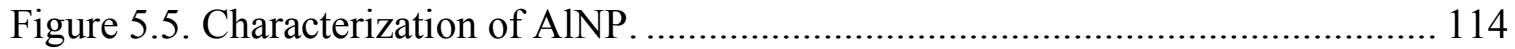

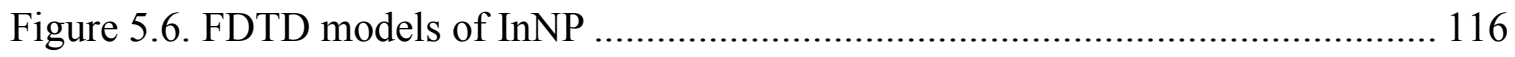

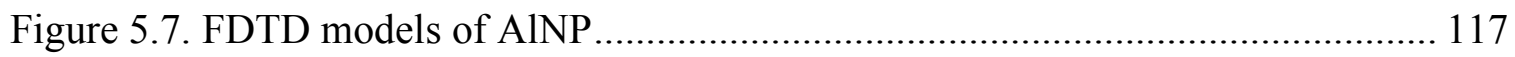




\section{List of Appendices}

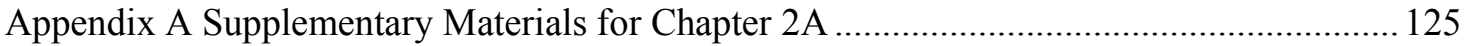

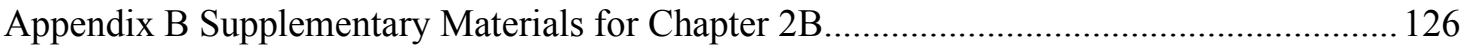

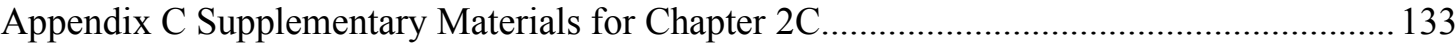

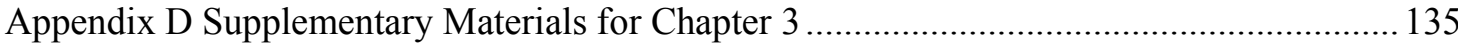

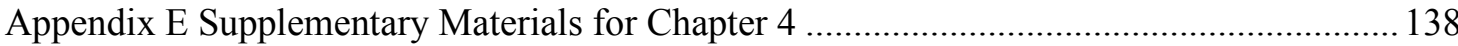

Appendix F Supplementary Materials for Chapter 5 ...................................................... 141

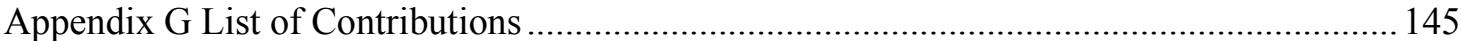




\title{
List of Abbreviations
}

\author{
Properties \\ DUV - Deep-ultraviolet \\ EM - Electromagnetic \\ LSPR - Localised Surface Plasmon Resonance \\ NIR - Near-infrared \\ $\varepsilon-$ Permittivity \\ $E,|E|,\left|E / E_{0}\right|-$ Electric field \\ $\lambda$-Wavelength \\ $\omega$ - Frequency \\ Materials and Chemicals \\ AgNP - Silver Nanoparticle \\ AgNC - Silver Nanocube \\ AINP - Aluminium Nanoparticle \\ InNP - Indium Nanoparticle \\ NPoM - Nanoparticle-over-mirror
}

\footnotetext{
Ala-Phe - Alanine-Phenylalanine

EG - Ethylene Glycol

PhTMS - Phenyltrimethoxysilane

PS - Polystyrene

PVP - Polyvinyl Pyrrolidone

Techniques and Characterization

AFM - Atomic Force Microscopy

FDTD - Finite-Difference Time-Domain

LB / LS - Langmuir-Blodgett / Langmuir-Schaeffer

SERS - Surface Enhanced Raman Spectroscopy

TEM - Transmission electron microscopy

UV-Vis - Ultraviolet - Visible
} 


\section{Functional Properties of Hybrid Resonances in Plasmonic Nanocrystals}

\section{Chapter 1: Introduction}

This work discusses the functional properties and applications of hybrid plasmonic modes in metal nanocrystals. Plasmonics is a field of study which explores the unique light-matter interactions in metallic nanostructures. These interactions stem from a property known as the surface-plasmon resonance. These surface plasmon resonances can strongly interact with their environment and with each-other, producing "hybrid" resonances with finely-tunable responses which can be exploited for a number of applications. This work explores the interactivity of surface plasmons and their functional properties, exploring how they can be utilized for a variety of purposes. The main hypothesis of the work is that hybridized modes can be used to expand the functionality of plasmonic materials in comparison to non-hybridized systems. 


\subsection{Fundamentals of Plasmonics}

The surface plasmon arises from the collective oscillation of electrons in the conduction band of a metal or other conductive material. This oscillation is initiated by displacement of the surface electrons by an external driving force, typically the electric field component of incident electromagnetic (EM) radiation. The movement of electrons generates electron-hole pairs resulting in Coulombic attraction to the nuclei of the metal creating a restoring force acting against the electric field.

In a bulk material or thin film, surface plasmons propagate as longitudinal waves on its surface which cannot couple directly to transverse EM radiation. High refractive index prisms can be used to match the momentum of the incident photons with the surface plasmon wave, allowing for the coupling between a surface plasmon and incident radiation producing a surface plasmon polariton (SPP). ${ }^{1}$

When the dimensions of the material are further confined in two or three dimensions, the surface plasmons can no longer propagate, resulting in the localized surface plasmon resonance (LSPR). This resonance is directly excitable by incident light and occurs in metal particles with dimensions equal to or smaller half its wavelength. In a LSPR the incident electric field displaces the surface-electrons in the metal sphere which subsequently experience a restoring force from the positive charges left behind. This in effect polarizes the metal particle, resulting in an enhanced electric field at the surface of the particle due to the build-up in charge density (Figure 1.1). When the exciting field oscillates at the same frequency as the frequency dictated by the restoring force, the excitation of the surface plasmon resonance occurs.
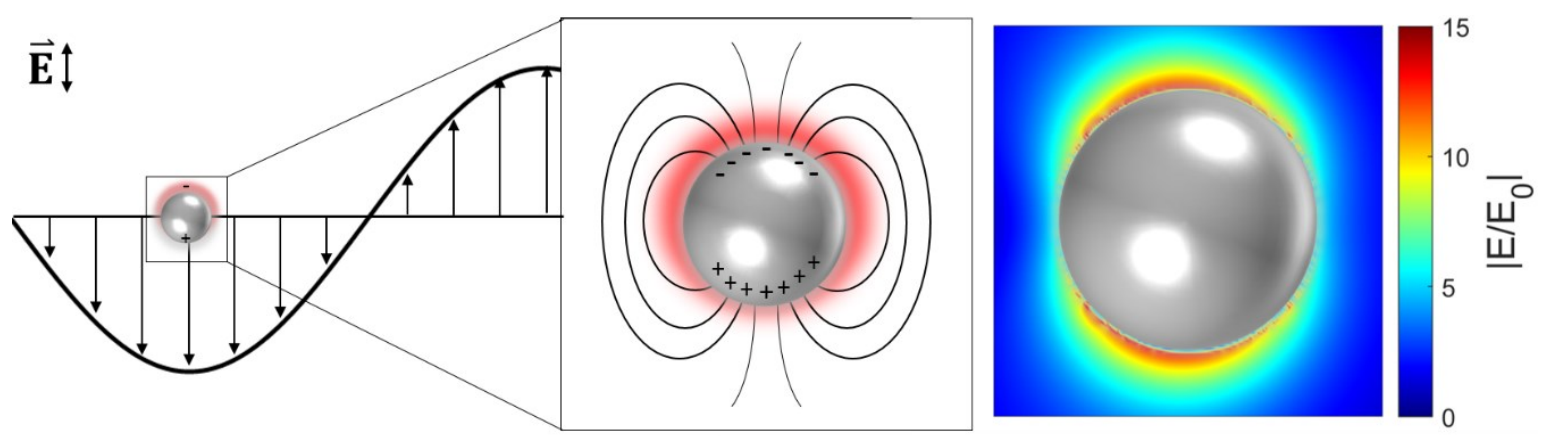

Figure 1.1. Schematic representation of the localized surface plasmon resonance (LSPR). An incident EM wave displaces the electron cloud, resulting in an oscillation around the particle and an enhanced electric field at the surface relative to the incident field. 
Gustav Mie's solutions to Maxwell's equations to describe the scattering of EM radiation by a sphere can be used to describe the extinction cross section $\left(C_{e x t}\right)$ of a metal sphere: ${ }^{2}$

$$
C_{e x t}=\frac{24 \pi^{2} r^{3} \epsilon_{m}^{\frac{3}{2}}}{\lambda}\left[\frac{\varepsilon_{i}}{\left(\varepsilon_{r}+2 \varepsilon_{m}\right)^{2}+\varepsilon_{i}{ }^{2}}\right]
$$

Where $r$ is the radius of the particle, $\lambda$ is the wavelength of incident EM radiation, $\varepsilon_{m}$ is the real dielectric function of the surrounding material, and $\varepsilon_{r}$ and $\varepsilon_{i}$ are the real and imaginary components of the complex dielectric function for the metal respectively. The extinction cross section is maximized when $\varepsilon_{r}=-2 \varepsilon_{m}$ when $\varepsilon_{i}$ is small, setting a resonance condition. This demonstrates a number of factors of fundamental importance to the position of the LSPR wavelength $\left(\lambda_{\mathrm{max}}\right)$ : it is dependent on the size of the particle $(r)$, the dielectric properties of the medium $\left(\varepsilon_{m}\right)$, and the dielectric properties of the plasmonic material itself $\left(\varepsilon_{r}\right.$ and $\left.\varepsilon_{i}\right)$.

\subsubsection{Materials for Plasmonics}

The resonance condition set by equation 1.1 demonstrates the importance in choice of material in plasmonic systems. In order for a material to support an LSPR or SPP the real part of the complex dielectric function, $\varepsilon_{r}$ must be large and negative, while it is desirable for the imaginary component, $\varepsilon_{r}$, to be small. The real component describes the polarizability of the material while the imaginary component describes absorption and losses in the material. These are both functions of frequency or wavelength, so the dielectric properties of the material indicate not only the ability to support a surface plasmon resonance but at what part of the EM spectrum they can be observed. The complex dielectric function for metals relevant to this work are displayed in Figure 1.2.3.4

The most common materials used in plasmonics are $\mathrm{Ag}$ and $\mathrm{Au}$, the choice of which is exemplified by their dielectric functions. The metals display an increasingly negative $\varepsilon_{r}$, as well as a minimal $\varepsilon_{i}$ above 300 and $500 \mathrm{~nm}$ for $\mathrm{Ag}$ and $\mathrm{Au}$ respectively. This yields high quality plasmons in the visible and NIR regions for both metals, though Au is limited to $>500 \mathrm{~nm}$ due to the high imaginary component at lower wavelengths caused by interband transitions in Au's d-band. ${ }^{5}$ Conversely In and Al have large imaginary components in the visible region but unlike $\mathrm{Ag}$ and $\mathrm{Au}$ they become minimal $<300 \mathrm{~nm}$, making them uniquely suitable for plasmonics in the UV region. 

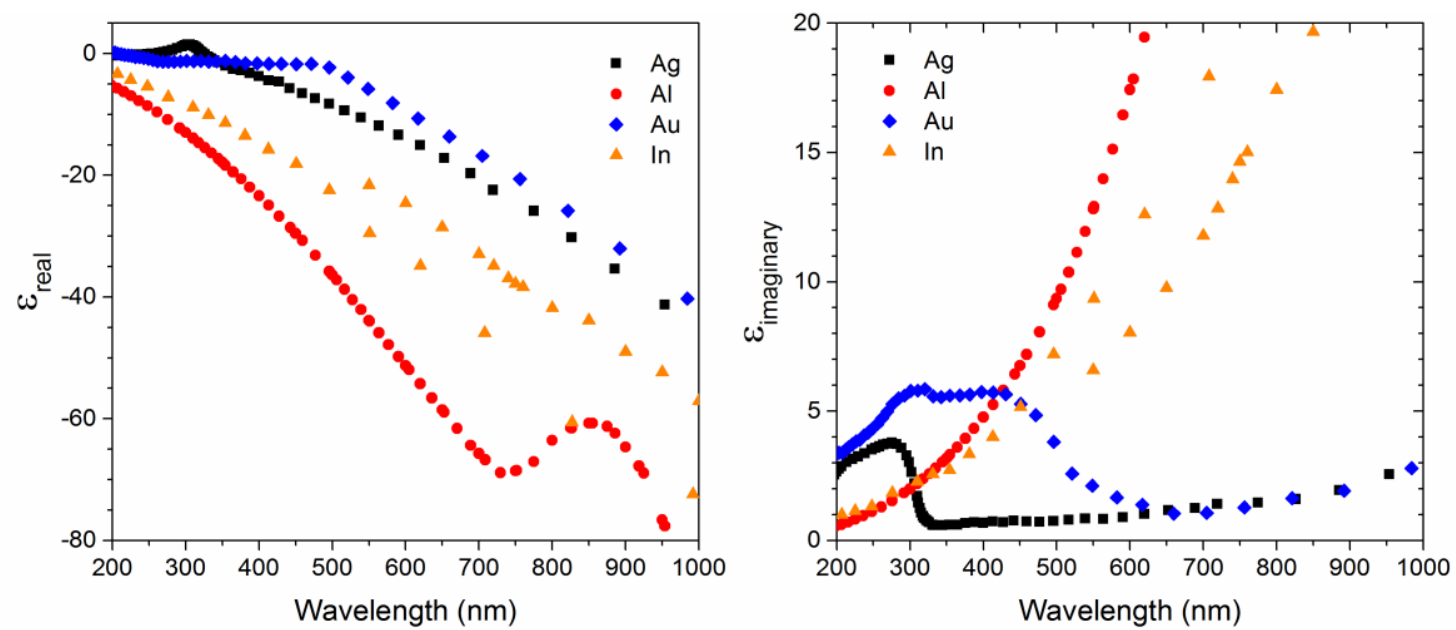

Figure 1.2. The real and imaginary components of the complex dielectric functions of relevant metals. 3,4 Strong plasmon resonances occur when the $\varepsilon_{\text {real }}$ is negative and $\varepsilon_{\text {imaginary }}<<\varepsilon_{\text {real. }}$.

This dependency on the dielectric functions of the material allows for the establishment of a quality factor, which loosely predicts the ability of a material to support a surface plasmon resonance: 6

$$
Q=\frac{-\varepsilon_{r}}{\varepsilon_{i}}
$$

While both $\mathrm{Ag}$ and $\mathrm{Au}$ demonstrate visible plasmon resonances in the visible region, $\mathrm{Ag}$ displays notably higher quality plasmons. The maximum Q of $\mathrm{Ag}$ is $97.43 @ 1083 \mathrm{~nm}$, and 33.99 @ $885 \mathrm{~nm}$. The higher $\varepsilon_{i}$ of $\mathrm{Au}$ in the visible region leads to broader resonances and a lower extinction efficiency with respect to Ag. While some other common metals display high quality surface plasmons, they make poor choices for applicable plasmonic materials. For example, $\mathrm{Na}$ displays a quality factor on par with Au at similar frequencies its significant reactivity hampers any practical use of the metal. Other common plasmonic materials include $\mathrm{Cu}, \mathrm{Pd}, \mathrm{Pt}$, and $\mathrm{Al},{ }^{6}$ all of which however have lower quality factors than $\mathrm{Ag}$ and $\mathrm{Au}$. Despite the superior quality and electromagnetic enhancement in Ag plasmonics, Au remains perhaps the most commonly used plasmonic material since $\mathrm{Ag}$ is susceptible to oxidation under ambient conditions. ${ }^{7} \mathrm{Au}$ is highly stable and biocompatible, making it ideal for biological or biomedical applications ${ }^{8}$ however it is also an expensive metal making alternatives to Au plasmonics attractive. ${ }^{5}$ 


\subsection{Modelling of Plasmonic Systems}

The Mie scattering equation (1.1) provides an exact solution for small nanopsheres in a homogeneous dielectric medium. However, functional plasmonic systems often contain multiple components in a variety of geometries which cannot be so easily described and more rigorous numerical calculations are required to model them. ${ }^{9}$ The ability to theoretically describe and model plasmonic structures is important for both the interpretation of experimental results as well as for the prediction of plasmonic behaviour. Modelling is an invaluable tool in examining properties of plasmonic nanostructures which are difficult to probe directly, such as electric field enhancement, leading to a significant degree of experimental-theoretical interaction in the field. The design of experimental systems or plasmonic architectures can also be accomplished by modelling, allowing an experimenter to determine optimum parameters such as the size and geometry of plasmonic system before fabricating it. In order to properly apply models, it is important to understand the theoretical basis, methods and limitations of them.

\subsubsection{Theoretical basis of modelling methods}

Plasmonic systems can be described in terms of classical electrodynamics, where the system can be described in terms of the propagation EM waves through different components and materials. The evolution of EM fields is determined by the Maxwell equations, which for a system containing no naturally magnetic components take on the form: ${ }^{10}$

$$
\begin{aligned}
& \frac{\partial \vec{E}}{\partial t}=\frac{1}{\varepsilon_{0}} \nabla \times \vec{H} \\
& \frac{\partial \vec{H}}{\partial t}=-\frac{1}{\mu_{0}} \nabla \times \vec{E}
\end{aligned}
$$

Where the electric and magnetic fields, $\vec{E}$ and $\vec{H}$ respectively, while $\varepsilon_{0}$ and $\mu_{0}$ are the permittivity and permeability of free space respectively, while $\varepsilon_{r}$ is the relative permittivity and is a function of frequency, $\omega$.

The challenge comes with solving these equations over an arbitrary space where the material and thus $\varepsilon_{r}(\omega)$ vary depending on spatial location. Typical techniques to solve an electrodynamic system thus involve discretizing the space and solving Maxwell's equations in these 
subsets of the space. This becomes numerically intensive to solve over large spaces and in three dimensions, but several computational techniques have been established to efficiently do so. One of the most used techniques is the finite-difference time-domain (FDTD) method, ${ }^{11}$ though other methods in the frequency domain such as the discrete dipole approximation (DDA) ${ }^{12}$ and finite element method (FEM) ${ }^{13}$ are also commonly used.

\subsubsection{Finite-difference time-domain calculations}

This work makes extensive use of the FDTD method. This is a robust method and wellestablished for modelling plasmonic systems..$^{9,14}$ There exist a variety of public domain and commercial FDTD solvers. This work uses the Lumerical FDTD solver as it contains convenient GUls, a number of useful features relative to plasmonics, and strong parallelization allowing efficient solving of large systems on multicore processors.

FDTD solvers work by discretizing the simulation space into a 2D or 3D mesh in cartesian coordinates, and then solving Maxwell's equations in each grid within the time domain. In actuality they use the following form of the equations: ${ }^{10}$

$$
\begin{aligned}
\frac{\partial \vec{D}}{\partial t} & =\nabla \times \vec{H} \\
\vec{D}(\omega) & =\varepsilon_{0} \varepsilon_{r}(\omega) \vec{E}(\omega) \\
\frac{\partial \vec{H}}{\partial t} & =-\frac{1}{\mu_{0}} \nabla \times \vec{E}
\end{aligned}
$$

where $\vec{D}$ is the displacement field. Note that $\vec{D}$ is written in the frequency domain, though must actually be written to the time domain for implementation in FDTD. ${ }^{10}$ Equations 1.4 is written as such however to demonstrate that each equation becomes interdependent and cyclic in this form, $(\vec{D}$ solves for $\vec{E}, \vec{E}$ solves for $\vec{H}, \vec{H}$ solves for $\vec{D}$ ) and the use of $\vec{D}$ allows for the implementation of the complex dielectric function $\varepsilon_{r}(\omega)$. The cyclic nature of these equations allows for the establishment of the Yee cell, ${ }^{15}$ in which solutions of the equations are propagated in time and space. In this scheme, the vectors of $E$ and $H$ are separated into six components $\left(\vec{E}_{x}, \vec{E}_{y}, \vec{E}_{z}\right.$, and $\vec{H}_{x}, \vec{H}_{y}, \vec{H}_{z}$ ), where the $\vec{E}$ and $\vec{H}$ components are discretized into separate grids which are offset 
by a half-step in time and space (Figure 1.3b). Propagation of the fields thus occurs by a 'leapfrog' method where $\vec{E}(t)$ solves for $\vec{H}(t)$ a half-step forward, and $\vec{H}(t)$ solves for $\vec{E}(t)$ to complete a full step. The number of cells determine the computational cost of the model.

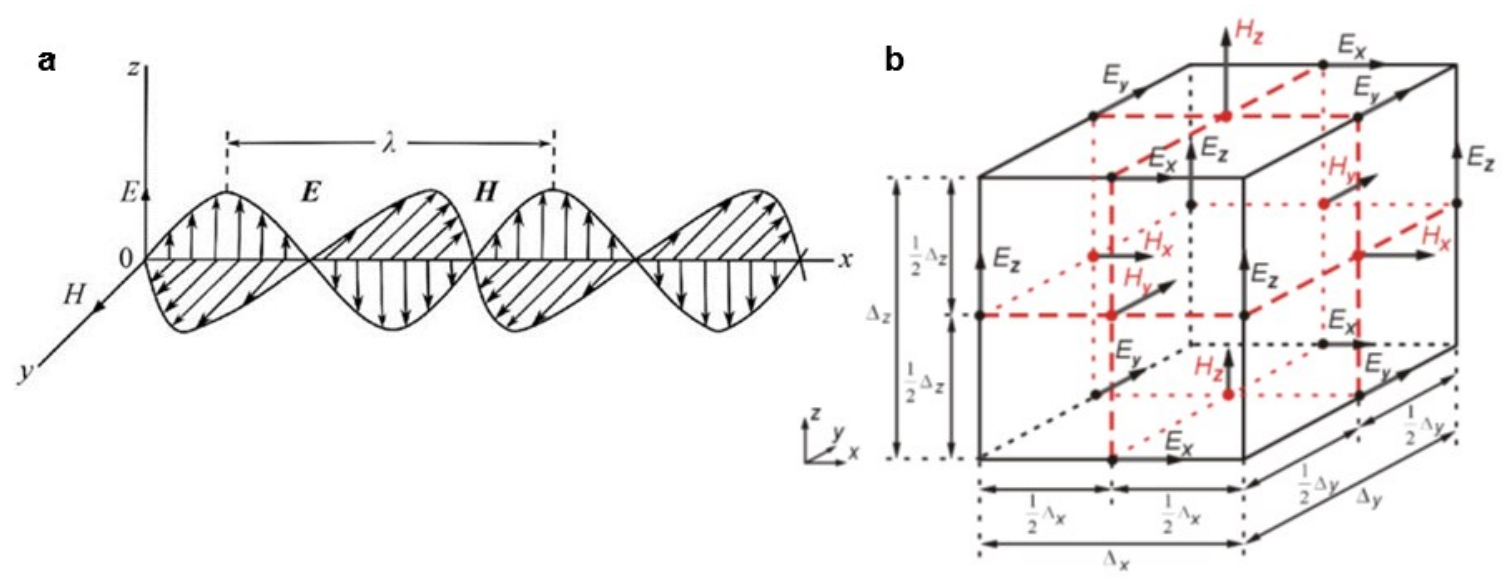

Figure 1.3. a) Schematic diagram of an electromagnetic wave showing the electric $(E)$ and magnetic $(H)$ components and $\mathbf{b}$ ) a diagram of the Yee cell, where $E(t)$ and $H(t)$ are offset in half-steps in order to propagate fields. Reproduced under open access CC license 4.0. ${ }^{16}$

The dielectric constant of most media varies by frequency, yet the computation is done in the time-domain, it is important to be able to simulate frequency-dependent materials, especially for plasmonic materials. The most common approach is to fit empirical dielectric constant data to an analytical form. This can then be sampled across the time domain. This poses a limitation to the method however, as in some cases the analytical models cannot be perfectly fitted to the known dielectric constants, introducing an inherent degree in error when describing the materials. ${ }^{9}$ The technique also cannot account for localized effects, such as changes in effective $\varepsilon_{r}$ at interfaces or in thin films; only the bulk permittivity is used.

The discretization of the simulation space in a cartesian grid also forms other limitations for describing plasmonic systems. For one it poses limitations in accurately describing the geometry of nanostructures, particularly those which are round or have rounded edges and corners. A very fine mesh must be used for the best description, but this is computationally costly This can be partially alleviated by using non-uniform meshes, ${ }^{17}$ and conformal mesh algorithms. ${ }^{18}$ The computational space becomes excessively large when trying to compute far-field effects and thus these cannot be probed directly. Since the size of the simulation space is limited appropriate 
boundaries must be set so that fields leaving the simulation space "disappear". Periodic boundaries or absorbing boundaries such as the perfectly matched layer (PML) are typically used for this. ${ }^{19}$

These limitations must be kept in mind when designing and performing FDTD simulations to avoid errors. To ensure accuracy of the simulation, they are often tested for "convergence," where the complexity of the model is gradually increased until it converges to a reproducible result, and/or is consistent with experimental observations. Despite the limitations, the direct numerical calculation of electric fields in optical system remains a useful tool for investigating and predicting the properties of plasmonic nanostructures.

\subsection{Tuning and Anisotropy in Plasmonic Systems}

The optical response of plasmonic nanostructures are very sensitive to numerous factors include the size, shape, and local environment. Significant control over plasmon frequencies, and the far-field and near-field properties of plasmonic nanoarchitectures is obtainable through their design and the manipulation of their local environment.

\subsubsection{Size and Shape}

Gustav Mie's solution for the sphere of an arbitrary size in equation (1.1) demonstrates the importance of the sphere's radius on the position and extinction cross section of a sphere. As a nanoparticle's size increases, the LSPR resonance will red-shift and broaden. ${ }^{20}$ As the particle's size increases the electron cloud experiences increasing depolarization, damping the electron oscillation to lower energies. ${ }^{21}$ In small particles, $(<30 \mathrm{~nm})$ only a single dipolar resonance is observable, a dipolar mode which oscillates parallel to the electric field. As the size of the particle increases, higher order modes, such as the quadrupolar mode where half the electron cloud moves parallel to the electric field and half moves anti-parallel, become important to the extinction profile of the particle. ${ }^{22}$ In some cases higher order modes may not display a net dipole moment and thus are not directly excited by incident light. ${ }^{23}$ These modes are referred to as dark modes and are still of importance as their charge distribution can heavily influence the near-field properties of nanostructures, ${ }^{24,25}$ and can be accessed by anisotropic environments or coupling to bright modes. ${ }^{26,27}$ 
The make-up of the extinction profile is also determined by size. The dipolar resonance of small particles is dominated by absorbance; they scatter very little light. However, as the particle size increases, the dipolar plasmon begins to scatter more light relative to its absorption, until scattering becomes the dominant contributor to extinction..$^{28,29}$

Changes to the plasmonic profile can also be influenced by the shape of the nanostructure. For example, in the case of an elongated particle such as a nanorod, the plasmonic signature is defined by a higher energy transverse plasmon which oscillates along the width of the structure, and a lower energy longitudinal plasmon which propagates along its length. ${ }^{30}$ Nanostructures with sharp edges or corners such as cubes, octahedrons and other polyhedrons also significantly alter the plasmonic profile compared to a spherical particle of similar size.${ }^{20}$ Charge accumulation at the corners and edges alter the spatial distribution and position of plasmonic modes; the dipolar mode in shaped particles will be redshifted compared to a spherical particle of the same diameter. The appearance of higher order modes is also magnified in shaped particles. For example, silver nanocubes begin supporting a high order mode at edge lengths of only $18 \mathrm{~nm} .{ }^{31}$

\subsubsection{Local environment}

The resonance condition set in the Mie scattering equation (1.1) establishes the importance of the dielectric constant of the medium, $\varepsilon_{m}$, in the maximal peak position of the LSPR. Using the resonance condition, $\varepsilon_{r}=-2 \varepsilon_{m}$, the max LSPR wavelength can be expressed as: ${ }^{32}$

$$
\lambda_{\max }=\lambda_{p} \sqrt{2 \varepsilon_{m}+1} \cong \lambda_{p} \sqrt{2} n_{m}
$$

where $\lambda_{p}$ is the plasma frequency of the bulk metal and $n_{\mathrm{m}}$ is the refractive index of the medium. The plasmon peak position $\lambda_{\max }$ is linearly dependent on the refractive index of the surrounding medium of the particle over small ranges of $n_{m}$. This relation has been experimentally verified, ${ }^{33}$ and forms the basis for localized surface plasmon resonance sensors. ${ }^{32}$ The LSPR and its sensitivity decay rapidly with distance from the nanoparticle surface. Shifts in the LSPR can be caused not only by a change in the bulk dielectric properties of the medium, but also in changes close to the surface of the particle. Alterations at the particle surface, such as the adsorption of molecules, ${ }^{34}$ or the formation of a self-assembled monolayer, ${ }^{35}$ produce sufficient change in the local environment to shift the plasmon resonance. 


\subsubsection{Anisotropic environments}

The localized sensitivity of plasmonic modes can produce peculiar effects if the particle is placed in an anisotropic environment. The breaking of symmetry in the environment of a plasmonic nanostructure, such as by the placement of a nanoparticle on a solid substrate, can produce unique interactions which redefine the plasmonic signature. The placement of a dielectric support is an important example, where the support enables plasmon modes to interact with their image charges in the dielectric, thereby forming new plasmon modes and redistributing the plasmon around the particle. ${ }^{36}$

This interaction can be explained by the plasmon hybridization model. ${ }^{37}$ Hybridization describes the interaction of plasmon modes in a "hybridization" scheme, analogous to molecular orbital theory, making for a convenient model for chemists. In this model two plasmon modes interact to form a "bonding" (symmetric) and "anti-bonding" (anti-symmetric) mode through constructive or destructive interference of the plasmonic modes. These resonances can also be calculated with electromagnetic theory, and ab initio techniques. ${ }^{37}$ The plasmon hybridization model allows for a convenient picture to display the energetics of plasmon mode-mixing The case for a plasmonic nanocube over a dielectric substrate is illustrated in Figure 1.4.
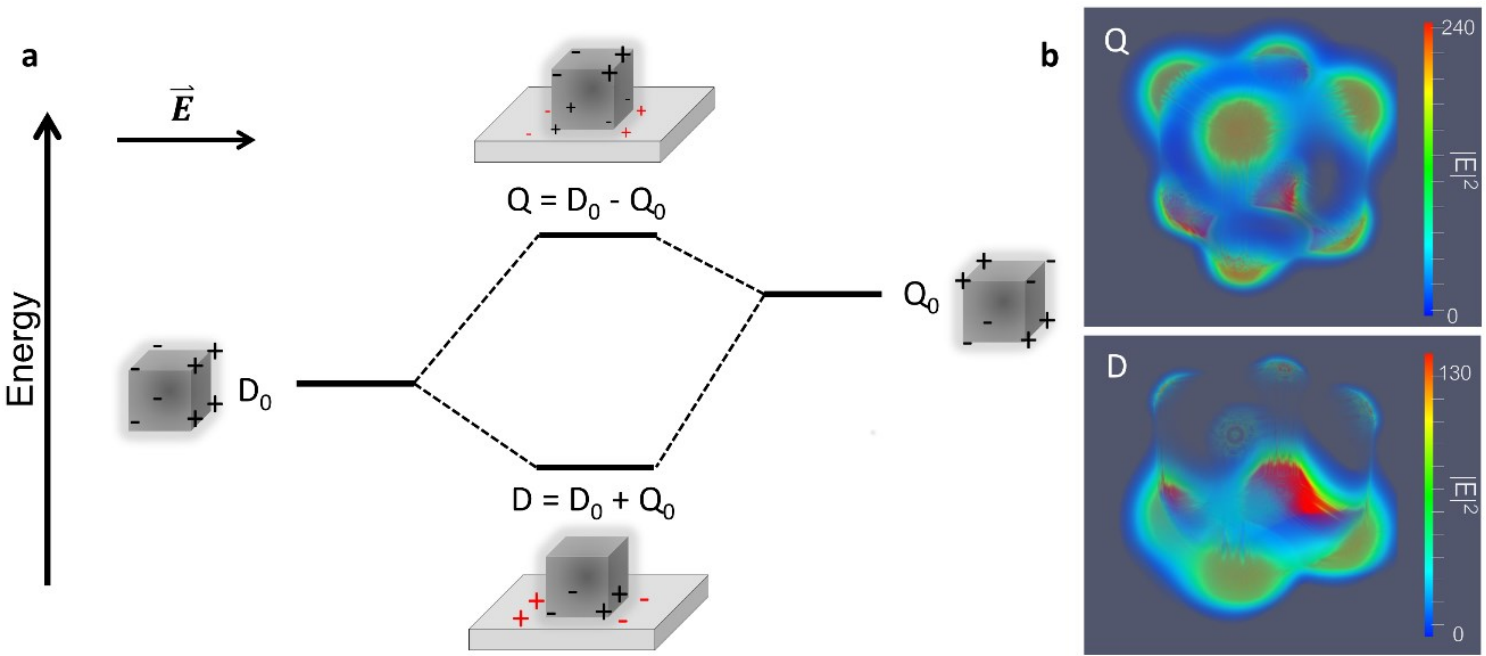

Figure 1.4. a) schematic representation of the substrate-induced plasmon hybridization scheme. The $D_{0}$ and normally dark Qo modes interact via image charges in the substrate to produce a "bonding" hybridized D and an "anti-bonding" $Q$ mode, which are separated in energy. The mixing is uneven; the $D$ and $Q$ modes retain dipolar and quadrupolar charge distributions respectively. b) 3D electric field $\left(|E|^{2}\right)$ data of a silver nanocube over a substrate from FDTD calculations, showing the modes are spatially separated as well; the D mode exists primarily in the high-index environment, and the $Q$ is strongest in the low-index environment. 
When brought in the vicinity or contact with a dielectric substrate, the dipolar $\left(D_{0}\right)$ and quadrupolar $\left(Q_{0}\right)$ modes interact via their image charges induced in the substrate. The $Q_{0}$ mode is typically dark in small cubes, it has no net dipole moment or a weak dipole moment in larger cubes. ${ }^{38}$ As these modes mix they produce a low energy hybrid, "bonding" dipolar mode (D), and a high energy "anti-bonding" quadrupolar mode $(Q)$, relative to the modes' initial energy. While both are a mixture of the $D_{0}$ and $Q_{0}$ modes, the hybrid $D$ mode is mostly dipolar in character as the nomenclature suggests, and likewise for the hybrid $Q$ mode..$^{39}$

An important consequence is not only are these modes now spectrally separated, but also spatially separated; the dipolar mode exists primarily in the substrate or higher index medium, whereas the quadrupolar mode is strongest in the low index medium. Silver nanocubes in particular display strong hybridization effects due to their propensity for supporting higher order modes which are nearly-degenerate,$^{39}$ and their geometry allows for high-area contact with planar substrates. ${ }^{40}$

\subsubsection{Plasmon coupling}

When two plasmonic nanoparticles are brought into the vicinity of each other a different form of mode-mixing occurs. Drastic colour changes have long been associated with the aggregation of colloidal nanoparticles, ${ }^{41}$ a property which forms the basis of colorimetric sensors, ${ }^{42}$ and plasmonic nanorulers. ${ }^{43}$ This interaction can be simply described as the coupling of two nearby oscillating dipoles to form a new resonant frequency. Depending on the relative polarization of the electric field the dipoles may couple in a transverse or longitudinal mode relative to the dimer axis, with the longitudinal interaction being the strongest.

Plasmon hybridization can again be used to describe the interaction, where the interaction of dipolar $\left(D_{0}\right)$, or higher order modes, can interact symmetrically or antisymmetrically to form "bonding" $\left(\mathrm{D}^{+}\right)$and "antibonding" ( $\left.\mathrm{D}^{-}\right)$modes respectively (Figure 1.5a). ${ }^{44}$ The anti-bonding mode has no net dipole moment and is a dark plasmon mode; it is not observable in far-field spectral measurements. ${ }^{25}$ Meanwhile the symmetric bonding mode produces a bright plasmonic mode with an observable spectral redshift relative to the original modes. The degree of splitting is relative to the interparticle distance, as it narrows the energy difference increases asymmetrically; the bonding mode shifts downwards in energy much faster than the anti-bonding mode increases in energy. 

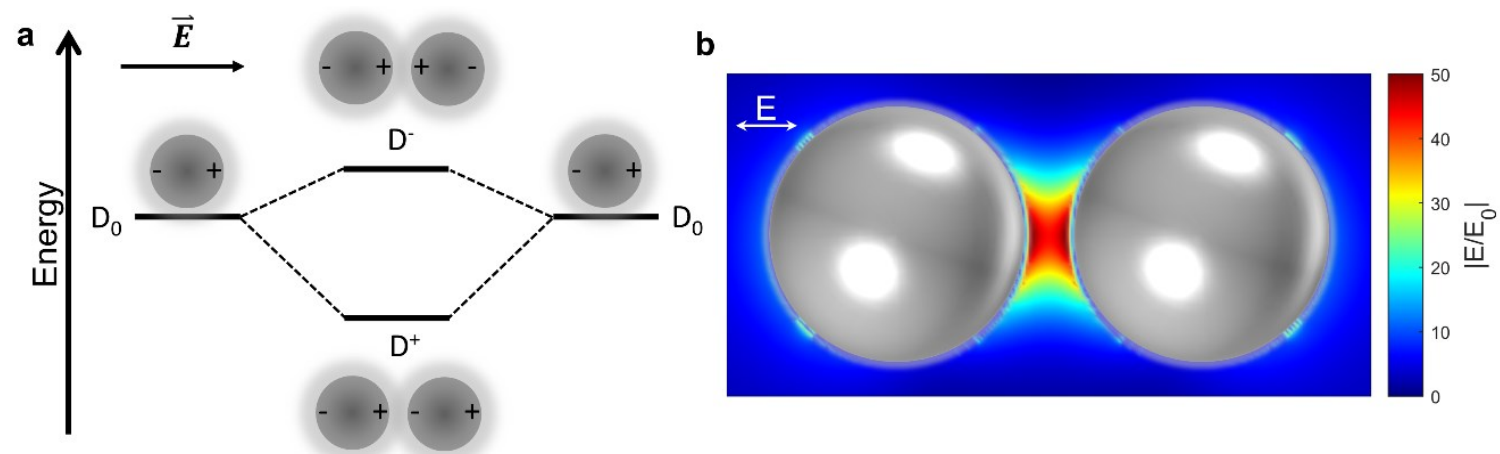

Figure 1.5. a) Plasmon hybridization model for a nanoparticle dimer. The particles' original dipolar modes $\left(D_{0}\right)$ interact to form a dark anti-bonding mode $\left(D^{-}\right)$, and bright bonding mode $\left(D^{+}\right)$, the latter produces an observable red-shift in the visible spectrum highly sensitive to inter-particle distance. b) The electric field in a nanoparticle dimer of the $D^{+}$mode is greatly intensified in the gap.

The coupling of plasmons between nanoparticles introduces a strong electric field enhancement in the junction between particles (Figure 1.5b). The field intensity tends to be $1-3$ orders of magnitude higher than that at the surface of individual particles. ${ }^{45}$ This near-field response becomes of crucial importance in applications such as surface-enhanced Raman spectroscopy (SERS). ${ }^{46}$ The spectral position and the electric field intensity coupled plasmon resonance is highly dependent on the inter-particle distance. ${ }^{47}$ The electric field intensity increases with decreasing gap size, approaching a maximum before conduction either via quantum tunnelling (at distances $<1$ $\mathrm{nm})$ or direct contact is made. ${ }^{48}$

\subsubsection{Gap-plasmons}

Plasmonic particles interact strongly with thin metal films leading to a coupling band between the film and the particle. The effect is very similar to inter-particle coupling and can also be described by the plasmon hybridization model, ${ }^{49}$ resulting in similar distance-dependent redshifts of the plasmon mode as it approaches the metal film. It can also be described in terms of a Fabry-Perot like cavity resonance; an optical resonator in a reflective cavity. ${ }^{50}$ In either description the position of the gap-plasmon is highly dependent on the particle-film distance and the dielectric medium between the two. ${ }^{51}$ The thickness of the metal film also influences the resonance; hybridization with SPR modes can enhance the response. ${ }^{22}$ Like inter-particle coupling, gap plasmons support highly enhanced electric-fields in the junction (Figure 1.6). 

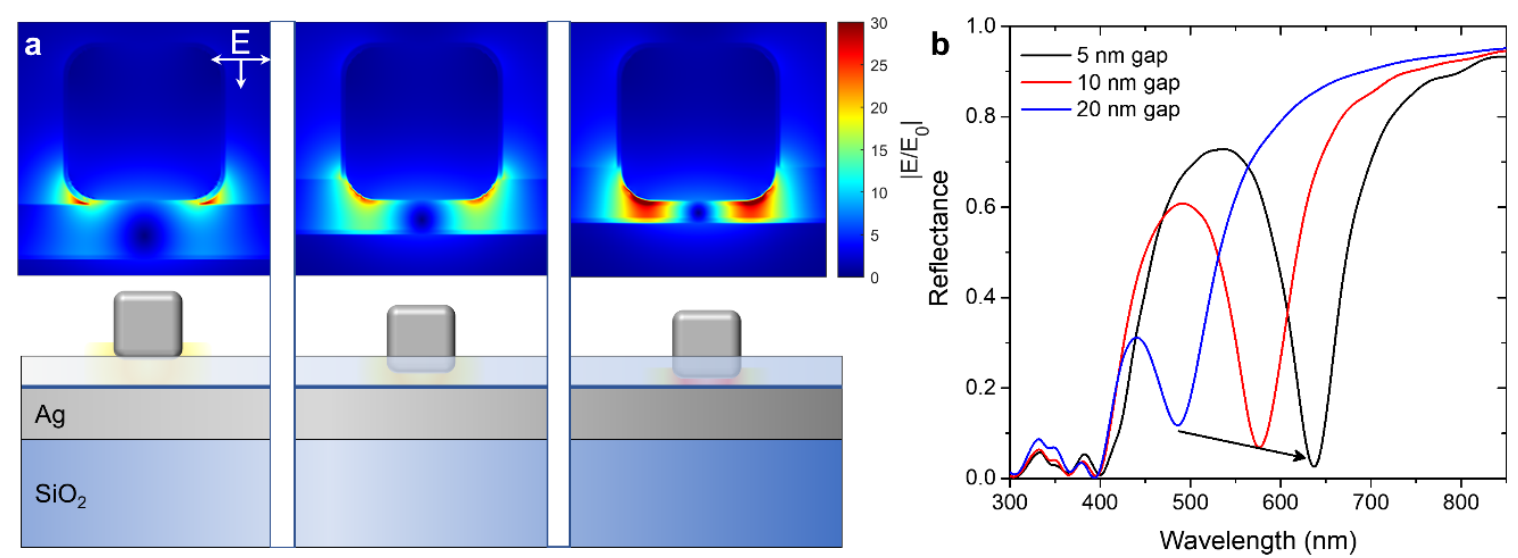

Figure 1.6. a) Electric field intensities of $60 \mathrm{~nm}$ Ag nanocubes over an $\mathrm{Ag}$ film separated by a dielectric spacer. The electric field intensity increases significantly when the particle is brought within $5 \mathrm{~nm}$ of the film. b) associated reflectance calculations showing a significant shift in the gap plasmon.

Gap plasmons are also highly absorptive, capable of making near-perfect absorbers,,$^{53}$ and they are also relatively angle and polarization insensitive. ${ }^{54}$ The high sensitivity of gap plasmons makes them attractive for a number of applications, such as in nanorulers, ${ }^{55}$ plasmon colour generation, ${ }^{56}$ and novel nano-optomechanical devices. ${ }^{57}$ The electric field enhancement in the cavity can be extreme,$^{58}$ which can be used for applications such as single-molecule detection by SERS. ${ }^{59}$

\subsection{Applications of Hybrid Plasmon Modes}

Several applications benefit from utilizing one or more types of the hybrid plasmonic modes described in section 1.3. The tunability of hybrid modes, as well as their unique far-field or nearfield optical properties can all be applied to techniques and functional materials in plasmonics. This section outlines some of the applications for plasmonics in the ultraviolet-visible spectrum that take advantage of or are otherwise influenced by hybrid plasmonic modes.

\subsubsection{Surface-Enhanced Raman Spectroscopy}

Surface-enhanced Raman spectroscopy (SERS) is a technique that falls under a family of field-enhanced phenomena, including surface-enhanced second harmonic generation, ${ }^{60}$ infrared absorption, ${ }^{61}$ and fluorescence. ${ }^{62}$ All of these techniques are reliant on the near-field optical enhancement near the surface of plasmonic nanostructures. The observation of SERS was first reported in the 1970's of Raman signals $10^{5}-10^{6}$ times higher than expected for molecules 
adsorbed onto roughened silver electrodes. ${ }^{63,64}$ It was some time before the initially unclear origins of the effect were attributed to the enhanced electric field at the surface of the electrodes. ${ }^{65}$ In the 1990's single-molecule detection with SERS was reported, ${ }^{66,67}$ and since then the technique has blossomed into a robust analytical technique used in areas such as biomedical, ${ }^{68}$ environmental, ${ }^{69}$ or forensic applications. ${ }^{70}$

Primary driver of the SERS phenomenon is enhanced electric field at surface of plasmonic nanostructures. Raman scattering is the inelastic scattering of an incident electromagnetic wave, at frequency $\omega_{0}$, by a polarizable molecular vibration occurring at $\omega_{\text {vib. }}$. The resulting scattered light occurs at $\left(\omega_{0}-\omega_{\text {vib }}\right)$ or $\left(\omega_{0}+\omega_{\text {vib }}\right)$ which correspond to Stokes and anti-Stakes Raman scattering respectively, the latter being energetically uphill and orders of magnitude less probable. ${ }^{71}$ The probability of Raman scattering is proportional to the square of the electric field intensity which the molecule experiences. ${ }^{72}$ Since a molecule is arbitrarily small in comparison to a metal nanoparticle, it benefits from the ability to experience the enhanced local electric field near the nanoparticle surface. The Raman scattered light may likewise be further enhanced by the nanoparticle by being elastically scattered into the far-field. The SERS enhancement $\left(G_{S E R S}\right)$ is thus dependent on the local field enhancement of both the incoming $E_{\text {in }}\left(\omega_{0}\right)$, and outgoing $E_{\text {out }}\left(\omega_{0}-\omega_{\text {vib }}\right)$ electric field. At visible frequencies, $\omega_{0} » \omega_{\text {vib }}$, so $|E|^{4}$ approximation is widely used to estimate SERS enhancement: ${ }^{73}$

$$
G_{S E R S}=\left|E_{\text {in }}\left(\omega_{0}\right)\right|^{2}\left|E_{\text {out }}\left(\omega_{0}-\omega_{\text {vib }}\right)\right|^{2} I_{0} \approx|E|^{4} I_{0}
$$

The intensity of the Raman signal thus benefits greatly by the enhanced electric fields at the surface nanoparticles, especially in hybridized structures which boast exception electric field enhancement such as nanoparticle clusters, and nanoparticle-over-metal films. ${ }^{74}$ The junctions of high electric field enhancement are often referred to as SERS "hot spots." ${ }^{75}$ The local $|E|^{4}$ enhancement in hotspots can easily exceed $10^{6}-10^{8}$, which is sufficient to enable single-molecule detection. ${ }^{76}$ Due to the large spatial invariance of local electric field strength, it is common for the majority of the SERS signal to be produced from a minority of the analyte in these hot spots. ${ }^{77}$

The electric field enhancement is not always the sole contributor to SERS enhancement. The chemical enhancement mechanism, in which charge transfer to the molecular species results 
in up to $10^{2}$ enhancement is sometimes observed. ${ }^{78}$ The Raman cross-section of an analyte is also naturally increased when the excitation frequency is close to that of an electronic transition in the molecule, known as the resonance-Raman effect. ${ }^{79}$ Vibrational modes which undergo a change in bond length during the excitation show an increase in polarizability and Raman cross section.

A common metric that is referred to in the evaluation of a SERS platform is the enhancement factor (EF). There exist a multitude of methods used experimentally to calculate the SERS EF and their implementation often inconsistent. ${ }^{80}$ Nonetheless the general definition of the enhancement factor is:

$$
\text { SERS EF }=\frac{I_{S E R S} / N_{S E R S}}{I_{\text {Raman }} / N_{\text {Raman }}}
$$

Where $I_{S E R S}$ and $I_{\text {Raman }}$ are the SERS and Raman intensities, and $N_{S E R S}$ and $N_{\text {Raman }}$ are the number of molecules measured in acquiring the SERS and Raman signals respectively. The quantification of $N$ tends to be the most challenging aspect of quantifying enhancement factors, it is common to overestimate $N_{S E R S}$, for example by assuming a closest packed monolayer at the nanostructure surface, so as not to overstate the enhancement factor. ${ }^{80}$

\subsubsection{Thermoplasmonics}

The stimulation of a plasmon resonance results in the excitation of electrons in the conduction band, which subsequently are subjected to electron-electron and electron-phonon collisions within the metal lattice. The energy absorbed by the nanoparticle is therefore thermally dissipated (Figure 1.7).
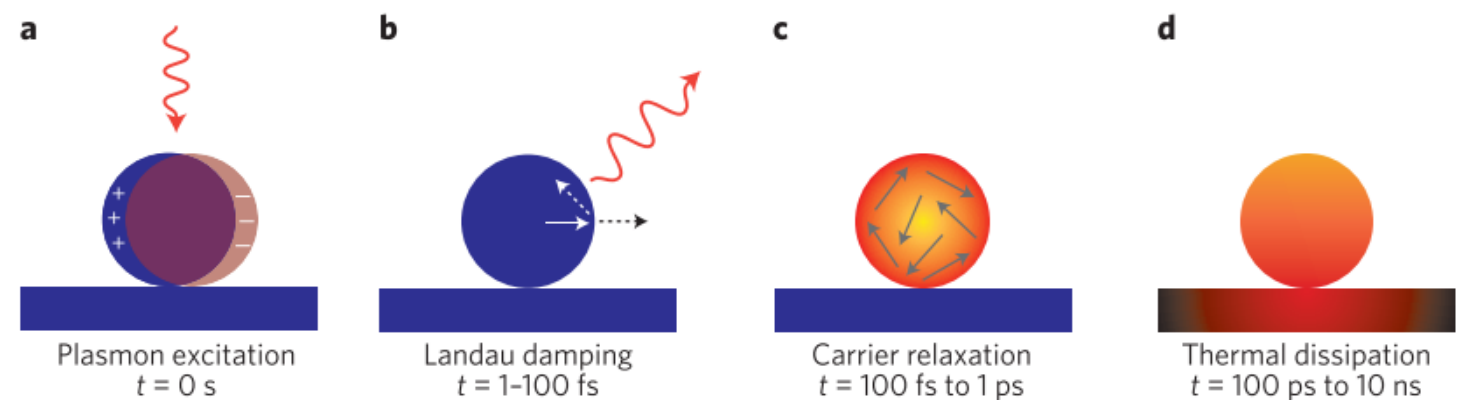

Figure 1.7. Schematic representation of the lifetime of a plasmon resonance. Upon the plasmon's excitation (a) the excited carriers undergo Landau damping (b) forming a thermal population of hot carriers (c) which subsequently relax and produce heat (d) through collisions in the lattice. Reproduced with permission, ${ }^{81} \odot$ Nature publishing group, 2015 
Plasmonic nanostructures thus produce heat on a rapid timescale, thermalizing on the order of picoseconds. ${ }^{82}$ The high thermal conductivity of the nanoparticles enables them to quickly dissipate this heat to their surroundings; they reach steady-state temperatures with their environment within nanoseconds. ${ }^{83,84}$ This is known as the thermoplasmonic effect and allows the nanoparticles to be used to localize and deliver heat to sub-diffraction limit dimensions. ${ }^{85}$ This phenomenon has been applied to areas such as photothermal therapeutics, ${ }^{86,87}$ photocatalysis, ${ }^{88,89}$ and solar thermal energy harvesting. ${ }^{90,91}$

The temperature increase of a plasmonic nanoparticle is dependent on the power absorbed by the nanoparticle $\left(P_{a b s}\right)$, and can be described by: ${ }^{92}$

$$
\Delta T_{n p}=\frac{P_{a b s}}{4 \pi r \kappa}=\frac{\sigma_{a b s} I}{4 \pi r \kappa}
$$

where $r$ is the radius of the nanoparticle and $\kappa$ is the thermal conductivity of the medium. $P_{a b s}$ can be defined by the irradiance of incident radiation, $l$, and its absorption cross section, $\sigma_{a b s}$. Thus, the degree of photothermal heating a nanoparticle experiences is directly proportional to its $\sigma_{a b s}$ at the excitation frequency. The exceptionally high $\sigma_{a b s}$ of plasmonic materials is in part what makes them so efficient at this process; the absorption cross section of a plasmonic nanoparticle can be several times larger than their physical cross section. ${ }^{93}$ It should be noted the absorption does not necessarily need to be plasmon-derived, for example interband transitions in gold nanoparticles can be excited to produce heat. ${ }^{85}$

Hybrid plasmonic modes can be used to fine tune the photothermal response of the plasmonic nanoparticles due to the dependency on $\sigma_{\text {abs }}$ on the temperature change. ${ }^{82}$ Plasmonic modes will have different relative contribution of scattering $\left(\sigma_{\text {scat }}\right)$ and absorbance to their extinction cross sections ( $\sigma_{\text {ext }}=\sigma_{a b s}+\sigma_{\text {scat }}$ ), depending on their size, shape and nature of the plasmonic mode. ${ }^{20}$ The thermoplasmonic effect can also be used to re-shape nanoparticles, significantly altering their plasmonic signature. ${ }^{85,94}$

\subsubsection{Plasmon Colour Generation}

The very earliest examples of plasmonics, such as the Lycurgus cup, ${ }^{95}$ medieval stained glass, ${ }^{96}$ or even Faraday's initial synthesis of colloidal gold, ${ }^{97}$ were admired for the fascinating colours they produced. While the origins were not understood at the time, plasmon-derived colours 
demonstrating properties such as dichroism and iridescence were valued for their beauty. The modern understanding of plasmonics has enabled the ability to finely tune the far-field optical response of plasmonic nanostructures, and produce wide palettes of plasmon-derived colours on demand ${ }^{56}$ Colours derived from nanostructures provide an alternative to dye-based pigments with potential for greater longevity, environmental sustainability and extreme resolution; plasmonic colours have been printed down to 100000 dots per inch (dpi), below the diffraction limit. $94,98,99$
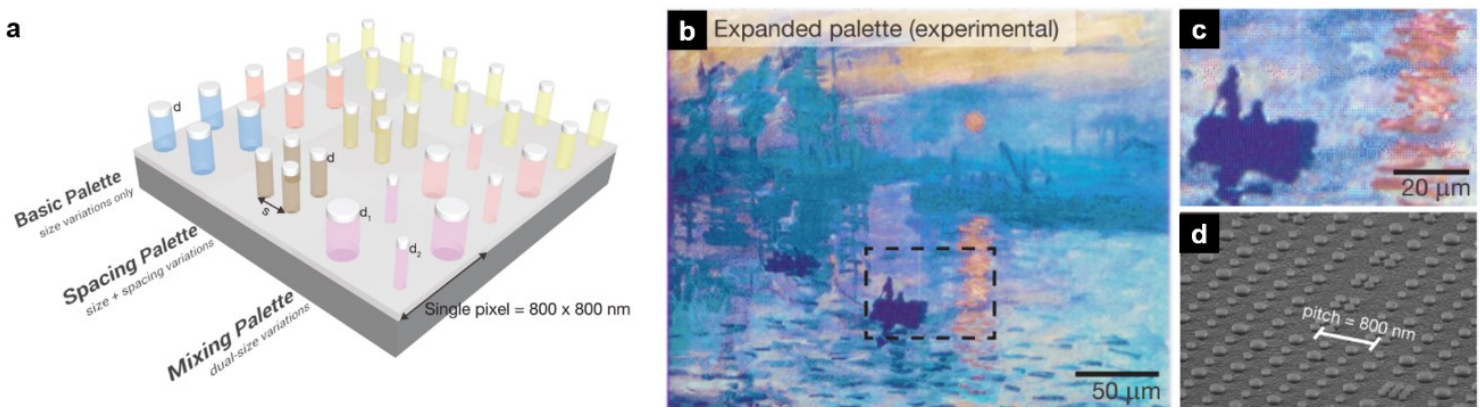

Figure 1.8. A plasmonic colour-printing scheme, a) the structure consists of Al nanodiscs placed above an Al film, by varying the size and spacing of the discs a wide palette of plasmonic pixels is produced $\mathbf{b}$ ) an image produced using the plasmonic structure, c) shown at higher magnification and d) under scanning electron microscopy. Reproduced with permission, ${ }^{100} \odot$ American Chemical Society, 2014.

Tuning of plasmonic colours is typically accomplished through means such as re-shaping the nanostructures,,$^{94}$ manipulation of their dielectric environment, ${ }^{101}$ or the use of hybrid plasmon modes. ${ }^{102}$ Using hybrid plasmon modes, either by coupling resonances from a disc and hole, ${ }^{102}$ or by using plasmon gap-plasmons, ${ }^{103}$ remain amongst the most promising methods for the facile generation of plasmon colours. The produced colours are generated by controlling the particle-film spacing with a dielectric spacer, and can be tuned across the entire visible spectrum due to the high sensitivity of these modes. ${ }^{104}$

\subsubsection{Photocatalysis}

Plasmons can be used to drive chemical reactions in multiple ways. Thermoplasmonics can be used to drive thermally activated chemical reactions and create local solvothermal effects. ${ }^{88}$ Electrons excited by plasmons can also directly be transferred to adsorbed surface molecules to drive chemical reactions. ${ }^{105}$ This pathway in particular has seen significant recent interest and arises from the ability the excited carriers generated upon the excitation of a plasmon, as previously shown in Figure 1.7, to transfer to molecular orbitals of molecules or valence and conduction bands of semiconductor photocatalysts in contact with the plasmonic structure. ${ }^{106}$ 
Plasmon driven photocatalysis is inherently linked to the near-field and far-field optical properties of the plasmonic nanostructures driving them. ${ }^{107}$ For example it has been demonstrated the number of "hot-electrons" which can be used to drive chemical reactions is proportional to the electric field enhancement of the system. ${ }^{108,109}$ Plasmonic photocatalysts also typically are employed as bi-functional system, often coupled with a semiconductor such as $\mathrm{TiO}_{2} .{ }^{110}$ As such manipulation of the plasmonic properties using their local environment can be beneficial to the catalytic efficiency of these devices such as by using anisotropic effects to tune the near-field distribution of the plasmon resonance.

\subsection{Experimental Methods}

\subsubsection{Synthesis of nanocrystals}

The synthesis of colloidal nanocrystals is typically initiated by the reduction of a metal ions $\left(\mathrm{M}^{\mathrm{x}}\right)$ by a reducing agent, or the thermal decomposition of organometallic compounds to continually produce zero-valent $\left(\mathrm{M}^{0}\right)$ metal to the reaction. As $\mathrm{M}^{0}$ is produced the solution is quickly saturated, owing to the low solubility of metals in common solvents, and once the concentration of $M^{0}$ reaches a critical concentration $\left(\mathrm{C}_{\text {crit }}\right)$, sufficient free energy exists to spontaneously form solid nuclei in the liquid environment (Figure 1.9). ${ }^{111}$

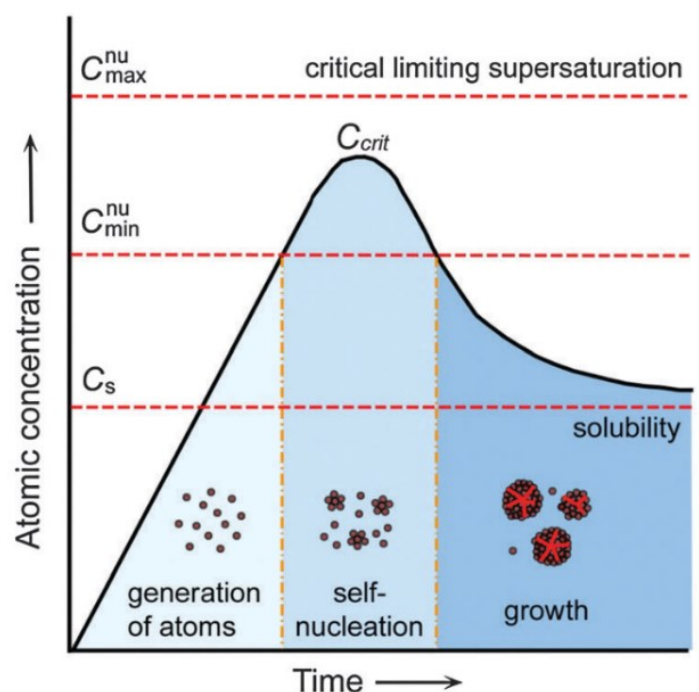

Figure 1.9. LaMer diagram of the growth process of colloidal crystals. After reduction of the precursor to $\mathrm{M}^{0}$ the solution becomes super-saturated with $\mathrm{M}^{0}$ initiating self-nucleation of the particles at a critical concentration ( $C_{\text {crit) }}$. Subsequent growth and agglomeration of the particles. Adapted, ${ }^{112} \odot$ American Chemical Society 1950, and reproduced with permission, ${ }^{113} @$ John Wiley and Sons 2009 
The formation of nuclei is followed by growth of the particle as the metal is reduced or accumulates on its surface. As the metal supply is consumed self-nucleation ceases and growth of existing nuclei becomes the sole fate of $\mathrm{M}^{0}$ atoms. When the $\mathrm{M}^{0}$ is reduced to the saturation concentration $\left(\mathrm{C}_{\mathrm{s}}\right)$ further growth of the nanocrystals occurs via aggregative growth of the existing particles, and Ostwald ripening. ${ }^{114}$

The synthesis of colloidal nanocrystals is done in the presence of some sort of surfactant or capping agent in order to stabilize them and prevent their agglomeration. These are typically adsorbed to the nanoparticle surface through electrostatic or Van der Waals interactions. ${ }^{113}$

\subsubsection{Shape-controlled synthesis of nanocrystals}

In addition to stabilizing the particle, the capping agent can serve an additional function: to direct the shape of the resultant nanoparticle. Since the properties of plasmonic systems are so highly sensitive to the shape and size over the particles used, control over these parameters in the synthesis of colloidal nanoparticles is valuable. Shape-controlled synthesis of nanocrystals is well developed for several metals such as $\mathrm{Pd},{ }^{115} \mathrm{Pt},{ }^{116} \mathrm{Au},{ }^{113}$ and $\mathrm{Ag} .{ }^{117}$ Shape-control is primarily achieved by choosing a capping agent that selectively binds to and passivates growth along a specific crystal facet. For example, silver nanocubes are produced by the selective passivation of the $\{100\}$ facets, allowing growth to occur primarily along the $\{111\}$ and $\{110\}$ directions. The result is a crystal with a high surface area of faces in the $\{100\}$ planes. Selection of the initial seed morphology is also crucial to the growth of the nanocrystal; silver nanocubes can only be grown from single crystal seeds. Defects in the crystal will result in alternate morphologies such as right bipyramids and nanowires, even with the same capping agent (Figure 1.10). ${ }^{118}$ Selection of the seed is either controlled kinetically as single crystals form faster than twinned crystals, ${ }^{119}$ though twinned seeds are preferred at slower reaction rates, ${ }^{120}$ or by oxidative etching where an etchant will react with high energy defect sites on the surface of twinned seeds. ${ }^{121}$ 


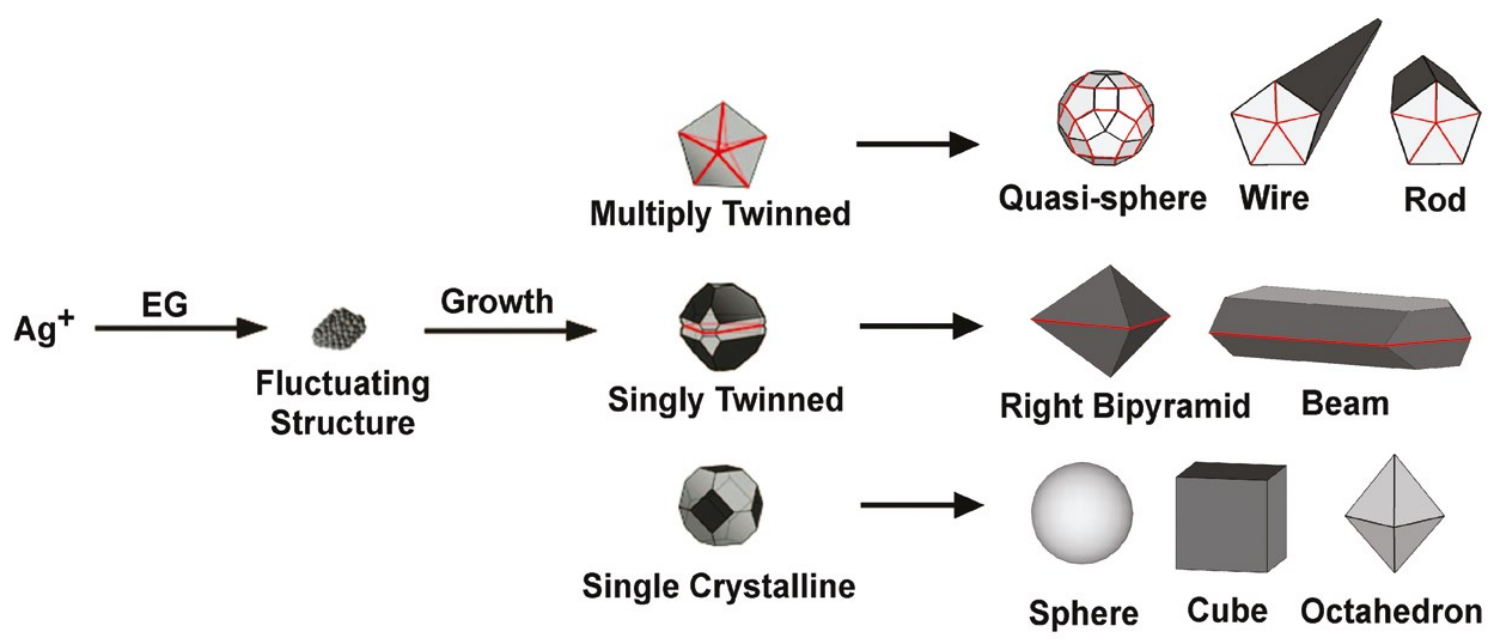

Figure 1.10. Shape-controlled synthesis of silver nanostructures. Ag nuclei will grow into either single crystals, or crystals containing single or multiple twin defects. Passivating agents can then be used to select the final morphology of the particle. Reproduced with permission, ${ }^{118}$ @ American Chemical Society, 2011

\subsubsection{Synthesis of silver nanocubes}

Silver nanocubes are used extensively in this work due to their strong hybridized plasmons. ${ }^{39}$ The synthesis of silver nanocubes is facile and reproducible using the polyol method. In this technique ethylene glycol (EG) is used as both the solvent and reducing agent, in the presence of polyvinylpyrrolidone (PVP). ${ }^{122,123}$ Heating of the ethylene glycol in the presence of $\mathrm{O}_{2}$ generates glycolaldehyde (GA), which is a stronger reducing agent than EG and will be the actual primary reductant in the reaction. ${ }^{124}$ As previously stated the cubic shape is selected from single seeds the preferential binding of PVP the $\{100\}$ facet, ${ }^{125}$ thermodynamically and kinetically limiting growth in this direction. ${ }^{126}$ Selection of single seeds can be done kinetically by using $\mathrm{Na}_{2} \mathrm{~S}$ to significantly improve the reduction rate of $\mathrm{Ag}^{+} .{ }^{119}$ Alternatively the presence of $\mathrm{Cl}^{-}$and $\mathrm{O}_{2}$, or other halides will oxidatively etch the seeds, slowing the reaction rate but increasing the preference for single crystals. ${ }^{121}$ Often a combination of these approaches are used, particularly in the synthesis of larger nanocubes. ${ }^{127}$

\subsubsection{Assembly of Colloidal Nanocrystals}

While initially produced as a colloidal suspension in solution phase, the properties and applicability of nanocrystals can be greatly enhanced by controlling their assembly in $1 \mathrm{D},{ }^{128} 2 \mathrm{D},{ }^{129}$ or 3D structures. ${ }^{130}$ The assembly of nanostructures is often performed through self-assembly: the spontaneous reorganization of the nanocrystals driven by the minimization of free energy in the 
system. ${ }^{131}$ This process can be influenced to produce a desired conformation of particles, such as by altering the capping agents, ${ }^{129}$, solvent-originated forces, ${ }^{132}$ or using a liquid/liquid or liquid/air interface. ${ }^{133}$

This work makes use of the Langmuir-Blodgett and Lanmguir-Schaeffer techniques to control the assembly of nanocrystals at an air-water interface..$^{134}$ In this technique the particles are deposited at the air-water interface to form a 2D Langmuir-film which can be controlled by a moveable barrier. This enables reproducible control over the density of the nanoparticle film. LanmguirBlodgett monolayers are controlled by an isothermal compression, such as in Figure 1.11.
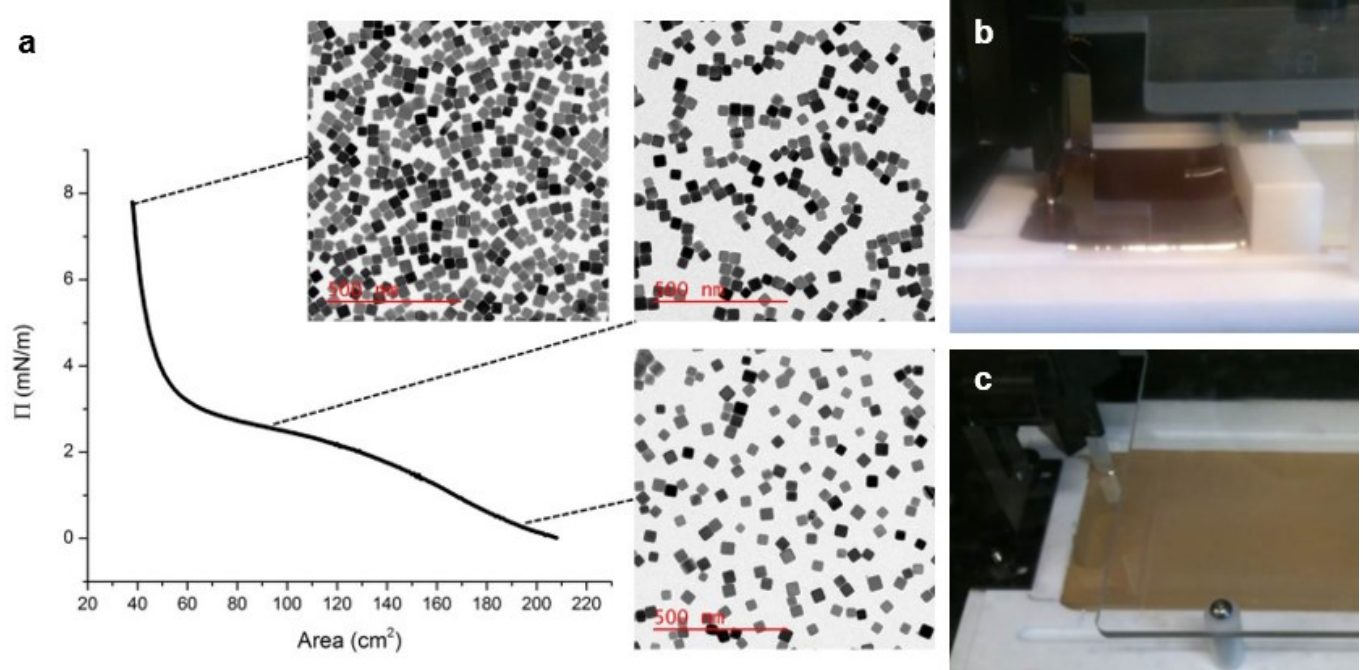

Figure 1.11. a) A Langmuir isotherm with TEM images of deposited nanocrystal assemblies at the gas phase $(\sim 0 \mathrm{mN} / \mathrm{m})$, liquid phase $(\sim 3 \mathrm{mN} / \mathrm{m})$ and solid phase $(\sim 8 \mathrm{mN} / \mathrm{m})$. Accompanying photographs of the layer at the b) solid and c) liquid phases.

Langmuir films are often characterized in terms of analogue phase. The gas-phase of the isotherm is characterized by a near-zero surface pressure and little sensitivity to compression and high mobility of components in the film. The liquid phase is indicated by a sloping isotherm, indicating compressibility of the layer though the mobility of the components is constrained. Lastly, the solid phase is characterized by incompressibility, reaching a near vertical slope in the pressure/area isotherm indicating maximum packing of the components. The Langmuir films can be transferred to a solid support using the Langmuir-Blodgett method, vertical dipping of the sample perpendicular to the plane of the monolayer, or the Langmuir-Schaeffer method, transferring the sample to parallel solid-support. The use of Langmuir films allows for careful control of the plasmonic properties of the assembly. 


\subsubsection{Characterization of colour}

For plasmonic materials with colour-based applications reliably being able to compare and quantify the colours produced is essential. Evaluating the spread, or gamut, of colours that can be produced is often done using the CIE (Commission international de l'eclairage) colourspace. The CIE 1931 colour space is a long-established international standard for colorimetry. Figure 1.12a displays the CIE colour space, as well as the sRGB colour space used in most displays and standard printing. Spectral data such as reflection, transmission or scattering measurements, can be transformed into the CIE colour space using tristimulus functions, $x, y, z$, which model the spectral sensitivity of the three types of human cone cells which perceive red, green and blue light respectively (Figure $1.12 b) .{ }^{135}$
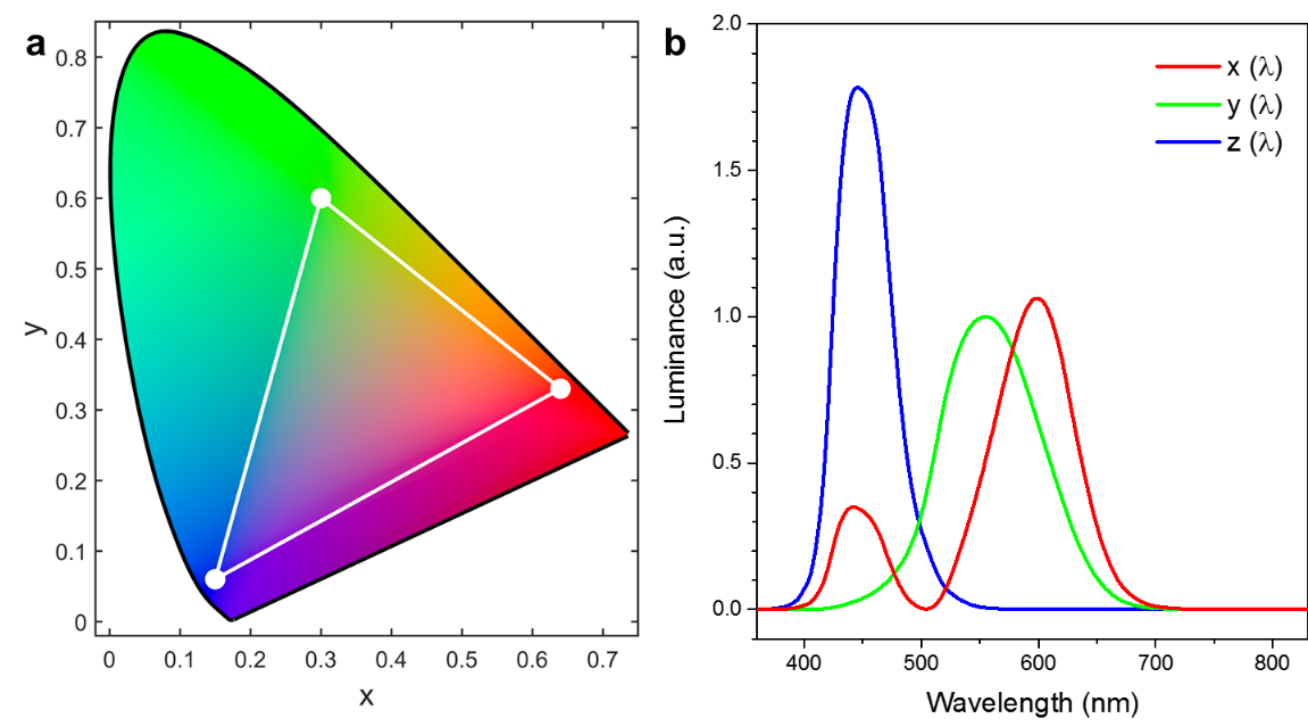

Figure 1.12. a) plot of the CIE 1931 colour space with, with the SRGB colour space shown within. Colours outside the triangle cannot be rendered properly on your display! b) The tristimulus colour matching functions, which model the sensitivity of the human eye to red, green and blue light.

The CIE colour space consists of three coordinates, $\mathrm{X}, \mathrm{Y}, \mathrm{Z}$, which correspond to the red, blue and green tristimulus response respectively. However, the values are typically normalized and displayed in 2 dimensions for ease of viewing, making the third coordinate redundant. ${ }^{136}$ As a result information on brightness is lost when using the $2 \mathrm{D}$ colour space. Spectral reflectance or transmittance data, $I(\lambda)$ can be converted to $\mathrm{CIE} X Y Z$ values using the colour matching functions: 


$$
\begin{aligned}
& X=\frac{1}{N} \int_{\lambda} S(\lambda) I(\lambda) \bar{x}(\lambda) d \lambda \\
& Y=\frac{1}{N} \int_{\lambda} S(\lambda) I(\lambda) \bar{y}(\lambda) d \lambda \\
& Z=\frac{1}{N} \int_{\lambda} S(\lambda) I(\lambda) \bar{z}(\lambda) d \lambda \\
& N=\int_{\lambda} I(\lambda) \bar{y}(\lambda) d \lambda
\end{aligned}
$$

Where $\bar{x}(\lambda), \bar{y}(\lambda)$, and $\bar{z}(\lambda)$ are the tristimulus functions, and $S(\lambda)$ is the illuminant function. The illuminant describes the light source of the viewed colours and is required with transmittive or reflective data as it provides a subjective bias to the observed colour. A number of standard illuminant functions exist to describe common light sources, but the most common used is the "D65" illuminant which simulates solar radiation with a colour temperature of $6500 \mathrm{~K}$ meant to mimic daylight conditions in the northern hemisphere accounting for atmospheric scattering.

\subsection{Rationale}

The properties of localised surface plasmon resonances in metal nanocrystals can be finely tuned by exploiting their high sensitivity to their vicinity. Hybridized plasmon modes allow for fine control of the far-field optical response and their near-field electromagnetic properties their spatial distribution. The following work studies the properties of and explores the use of hybrid plasmonic modes in a number of systems.

Chapter 2 uses theoretical modelling to elucidate the far-field and near-field optical properties of hybrid plasmon modes in three distinct anisotropic systems involving silver nanocubes. The modelling is used to show how the spatial distribution of the electric field near hybridized plasmon modes affects the far-field optical response which is observed experimentally. These include:

A) A nano-ruler, where the spatially separated modes are used to track the movement of a nanocube in an anisotropic environment with nanometer-precision; 
B) An unusually sharp plasmonic response observed in a system, where thin dielectric coatings are used to shift the environment from an asymmetric one to a symmetric one, though the slight anisotropy produces a constructive interference.

C) A set of anisotropic colloidal core@half-shell particles, where the spatially separated plasmon modes can potentially be leveraged in sensing and photocatalytic applications

Chapter 3 expands upon the technique explored in Chapter 2A), where photothermal effects are used to locally apply heat and move nanocubes in their anisotropic environment. Hybridized modes allow for the characterization of the response, benefit the sensitivity of the LSPRshifts, and can enable routes to further improve the technique. Chapter 4 explores one of these such improvements by using hybridized gap-plasmons to produce wide LSPR shifts to create a palette of plasmon-derived colours.

Finally, Chapter 5 explores the use of alternative plasmonic materials to access the UV region of the electromagnetic spectrum. The use of the nanocrystals synthesized here in deep-UV SERS is investigated. SERS is a technique which inherently relies on the strong electric fields produced in "hot spots" in hybridized plasmonic structures. It should be noted that, chronologically, this work was performed before the work presented in other chapters, and a discussion of how recent advances in the field and utilizing hybrid-plasmon modes could further improve the technique is included.

Overall this work aims to demonstrate how the manipulation of the far-field and near-field optical properties of plasmonic nanostructures can be used to enhance their functional properties. 


\subsection{References}

(1) Barnes, W. L.; Dereux, A.; Ebbesen, T. W. Surface Plasmon Subwavelength Optics. Nature 2003, 424 (6950), 824-830.

(2) Mulvaney, P. Surface Plasmon Spectroscopy of Nanosized Metal Particles. Langmuir 1996, 12 (3), 788-800.

(3) Palik, E. D. Handbook of Optical Constants of Solids; 1985; Vol. 1.

(4) Johnson, P. B.; Christy, R. W. Optical Constants of the Noble Metals. Phys. Rev. B 1972 , 6 (12), 4370-4379.

(5) West, P. R.; Ishii, S.; Naik, G. V.; Emani, N. K.; Shalaev, V. M.; Boltasseva, A. Searching for Better Plasmonic Materials. Laser Photonics Rev. 2010, 4 (6), 795-808.

(6) Blaber, M. G.; Arnold, M. D.; Ford, M. J. A Review of the Optical Properties of Alloys and Intermetallics for Plasmonics. J. Phys. Condens. Matter 2010, 22 (14), 143201.

(7) Graedel, T. E. Corrosion Mechanisms for Silver Exposed to the Atmosphere. J. Electrochem. Soc. 1992, 139 (7), 1963.

(8) Khlebtsov, N. G.; Dykman, L. a. Optical Properties and Biomedical Applications of Plasmonic Nanoparticles. J. Quant. Spectrosc. Radiat. Transf. 2010, 111 (1), 1-35.

(9) Gray, S. K. Theory and Modeling of Plasmonic Structures. J. Phys. Chem. C 2013, 117 (5), 1983-1994.

(10) Sullivan, D. M. Electromagnetic Simulation Using the FDTD Method, 2nd ed.; IEEE Press, 2013.

(11) Zhao, J.; Pinchuk, A. O.; McMahon, J. M.; Li, S.; Ausman, L. K.; Atkinson, A. L.; Schatz, G. C. Methods for Describing the Electromagnetic Properties of Silver and Gold Nanoparticles. Acc. Chem. Res. 2008, 41 (12), 1710-1720.

(12) Draine, B. T.; Flatau, P. J. Discrete-Dipole Approximation For Scattering Calculations. J. Opt. Soc. Am. A 1994, 11 (4), 1491.

(13) Khoury, C. G.; Norton, S. J.; Vo-Dinh, T. Investigating the Plasmonics of a Dipole-Excited Silver Nanoshell: Mie Theory versus Finite Element Method. Nanotechnology 2010, 21 (31).

(14) Montgomery, J. M.; Lee, T. W.; Gray, S. K. Theory and Modeling of Light Interactions with Metallic Nanostructures. J. Phys. Condens. Matter 2008, 20 (32).

(15) Kane Yee. Numerical Solution of Initial Boundary Value Problems Involving Maxwell's Equations in Isotropic Media. IEEE Trans. Antennas Propag. 1966, 14 (3), 302-307.

(16) Choroszucho, A. Analysis of the Influence of the Complex Structure of Clay Hollow Bricks on the Values of Electric Field Intensity by Using the FDTD Method. Arch. Electr. Eng. 2016, 65 (4), 745-759.

(17) Chevalier, M. W.; Luebbers, R. J.; Cable, V. P. FDTD Local Grid with Material Traverse. IEEE Trans. Antennas Propag. 1997, 45 (3), 411-421.

(18) Yu, W.; Mittra, R. A Conformal Finite Difference Time Domain Technique for Modeling Curved Dielectric Surfaces. IEEE Microw. Wirel. Components Lett. 2001, 11 (1), 25-27.

(19) Gedney, S. D. Introduction to the Finite-Difference Time-Domain (FDTD) Method for Electromagnetics; Morgan \& Claypool, 2011.

(20) Noguez, C. Surface Plasmons on Metal Nanoparticles: The Influence of Shape and Physical Environment. J. Phys. Chem. C 2007, 111 (10), 3806-3819.

Meier, M.; Wokaun, A. Enhanced Fields on Large Metal Particles: Dynamic 
Depolarization. Opt. Lett. 1983, 8 (11), 581-583.

(22) Kelly, K.; Coronado, E. The Optical Properties of Metal Nanoparticles: The Influence of Size, Shape, and Dielectric Environment. J. Phys. Chem. B 2003, 107, 668-677.

(23) Gómez, D. E.; Teo, Z. Q.; Altissimo, M.; Davis, T. J.; Earl, S.; Roberts, a. The Dark Side of Plasmonics. Nano Lett. 2013, 13 (8), 3722-3728.

(24) Herzog, J. B.; Knight, M. W.; Li, Y.; Evans, K. M.; Halas, N. J.; Natelson, D. Dark Plasmons in Hot Spot Generation and Polarization in Interelectrode Nanoscale Junctions. Nano Lett. 2013, 13 (3), 1359-1364.

(25) Barrow, S. J.; Rossouw, D.; Funston, A. M.; Botton, G. A.; Mulvaney, P. Mapping Bright and Dark Modes in Gold Nanoparticle Chains Using Electron Energy Loss Spectroscopy. Nano Lett. 2014, 14 (7), 3799-3808.

(26) Schertz, F.; Schmelzeisen, M.; Mohammadi, R.; Kreiter, M.; Elmers, H.-J.; Schönhense, G. Near Field of Strongly Coupled Plasmons: Uncovering Dark Modes. Nano Lett. 2012, 12 (4), 1885-1890.

(27) Zheng, Z.; Huang, B.; Qin, X.; Zhang, X.; Dai, Y.; Whangbo, M.-H. Facile in Situ Synthesis of Visible-Light Plasmonic Photocatalysts M@TiO2 (M = Au, Pt, Ag) and Evaluation of Their Photocatalytic Oxidation of Benzene to Phenol. J. Mater. Chem. 2011, 21 (25), 9079.

(28) Jain, P. K.; Lee, K. S.; El-Sayed, I. H.; El-Sayed, M. A. Calculated Absorption and Scattering Properties of Gold Nanoparticles of Different Size, Shape, and Composition: Applications in Biological Imaging and Biomedicine. J. Phys. Chem. B 2006, 110 (14), 7238-7248.

(29) Evanoff, D. D.; Chumanov, G. Size-Controlled Synthesis of Nanoparticles. 2. Measurement of Extinction, Scattering, and Absorption Cross Sections. J. Phys. Chem. B 2004, 108 (37), 13957-13962.

(30) Wang, H.; Brandl, D. W.; Le, F.; Nordlander, P.; Halas, N. J. Nanorice: A Hybrid Plasmonic Nanostructure. Nano Lett. 2006, 6 (4), 827-832.

(31) Wang, Y.; Zheng, Y.; Huang, C. Z.; Xia, Y. Synthesis of Ag Nanocubes 18-32 Nm in Edge Length: The Effects of Polyol on Reduction Kinetics, Size Control, and Reproducibility. J. Am. Chem. Soc. 2013, 135 (5), 1941-1951.

(32) Mayer, K. M.; Hafner, J. H. Localized Surface Plasmon Resonance Sensors. Chem. Rev. 2011, 111 (6), 3828-3857.

(33) Ahamad, N.; Bottomley, A.; lanoul, A. Optimizing Refractive Index Sensitivity of Supported Silver Nanocube Monolayers. J. Phys. Chem. C 2012, 116 (1), 185-192.

Yonzon, C. R.; Jeoung, E.; Zou, S.; Schatz, G. C.; Mrksich, M.; Van Duyne, R. P. A Comparative Analysis of Localized and Propagating Surface Plasmon Resonance Sensors: The Binding of Concanavalin A to a Monosaccharide Functionalized SelfAssembled Monolayer. J. Am. Chem. Soc. 2004, 126 (39), 12669-12676.

(35) Haes, A. J.; Zou, S.; Schatz, G. C.; Van Duyne, R. P. A Nanoscale Optical Biosensor: The Long Range Distance Dependence of the Localized Surface Plasmon Resonance of Noble Metal Nanoparticles. J. Phys. Chem. B 2004, 108 (1), 109-116.

(36) Knight, M. W.; Wu, Y.; Lassiter, J. B.; Nordlander, P.; Halas, N. J. Substrates Matter: Influence of an Adjacent Dielectric on an Individual Plasmonic Nanoparticle. Nano Lett. 2009, 9 (5), 2188-2192.

(37) Prodan, E.; Radloff, C.; Halas, N. J.; Nordlander, P. A Hybridization Model for the Plasmon Response of Complex Nanostructures. Science (80-. ). 2003, 302 (5644), 419422. 
(38) Ringe, E.; McMahon, J. M.; Sohn, K.; Cobley, C.; Xia, Y.; Huang, J.; Schatz, G. C.; Marks, L. D.; Van Duyne, R. P. Unraveling the Effects of Size, Composition, and Substrate on the Localized Surface Plasmon Resonance Frequencies of Gold and Silver Nanocubes: A Systematic Single-Particle Approach. J. Phys. Chem. C 2010, 114 (29), 12511-12516.

(39) Zhang, S.; Bao, K.; Halas, N. J.; Xu, H.; Nordlander, P. Substrate-Induced Fano Resonances of a Plasmonic Nanocube: A Route to Increased-Sensitivity Localized Surface Plasmon Resonance Sensors Revealed. Nano Lett. 2011, 11 (4), 1657-1663.

(40) Albella, P.; Garcia-Cueto, B.; González, F.; Moreno, F.; Wu, P. C.; Kim, T. H.; Brown, A.; Yang, Y.; Everitt, H. O.; Videen, G. Shape Matters: Plasmonic Nanoparticle Shape Enhances Interaction with Dielectric Substrate. Nano Lett. 2011, 11 (9), 3531-3537.

(41) Halas, N. J.; Lal, S.; Chang, W.; Link, S.; Nordlander, P. Plasmons in Strongly Coupled Metallic Nanostructures. Chem. Rev. 2011, 111 (6), 3913-3961.

(42) Wang, Z.; Ma, L. Gold Nanoparticle Probes. Coord. Chem. Rev. 2009, 253 (11-12), 1607-1618.

(43) Sönnichsen, C.; Reinhard, B. M.; Liphardt, J.; Alivisatos, a P. A Molecular Ruler Based on Plasmon Coupling of Single Gold and Silver Nanoparticles. Nat. Biotechnol. 2005, 23 (6), 741-745.

(44) Nordlander, P.; Oubre, C.; Prodan, E.; Li, K.; Stockman, M. I. Plasmon Hybridization in Nanoparticle Dimers. Nano Lett. 2004, 4 (5), 899-903.

(45) Sweatlock, L.; Maier, S.; Atwater, H.; Penninkhof, J.; Polman, a. Highly Confined Electromagnetic Fields in Arrays of Strongly Coupled Ag Nanoparticles. Phys. Rev. B 2005, 71 (23), 235408.

(46) Xu, H.; Aizpurua, J.; Kall, M.; Apell, P. Electromagnetic Contributions to Single-Molecule Sensitivity in Surface-Enhanced Raman Scattering. Phys. Rev. E 2000, 62 (3 Pt B), 43184324.

(47) Zuloaga, J.; Prodan, E.; Nordlander, P. Quantum Description of the Plasmon Resonances of a Nanoparticle Dimer. Nano Lett. 2009, 9 (2), 887-891.

(48) Esteban, R.; Borisov, A. G.; Nordlander, P.; Aizpurua, J. Bridging Quantum and Classical Plasmonics with a Quantum-Corrected Model. Nat. Commun. 2012, 3 (May), 825.

(49) Nordlander, P.; Prodan, E. Plasmon Hybridization in Nanoparticles near Metallic Surfaces. Nano Lett. 2004, 4 (11), 2209-2213.

(50) Søndergaard, T.; Bozhevolnyi, S. Slow-Plasmon Resonant Nanostructures: Scattering and Field Enhancements. Phys. Rev. B - Condens. Matter Mater. Phys. 2007, 75 (7), 1-4.

(51) Baumberg, J. J.; Aizpurua, J.; Mikkelsen, M. H.; Smith, D. R. Extreme Nanophotonics from Ultrathin Metallic Gaps. Nat. Mater. 2019.

(52) Ding, F.; Yang, Y.; Deshpande, R. A.; Bozhevolnyi, S. I. A Review of Gap-Surface Plasmon Metasurfaces: Fundamentals and Applications. Nanophotonics 2018, 7 (6), 1129-1156.

(53) Akselrod, G. M.; Huang, J.; Hoang, T. B.; Bowen, P. T.; Su, L.; Smith, D. R.; Mikkelsen, M. $\mathrm{H}$. Large-Area Metasurface Perfect Absorbers from Visible to Near-Infrared. Adv. Mater. 2015, 27 (48), 8028-8034.

(54) Moreau, A.; Ciracì, C.; Mock, J. J.; Hill, R. T.; Wang, Q.; Wiley, B. J.; Chilkoti, A.; Smith, D. R. Controlled-Reflectance Surfaces with Film-Coupled Colloidal Nanoantennas. Nature 2012, 492 (7427), 86-89.

(55) Hill, R. T.; Kozek, K. M.; Hucknall, A.; Smith, D. R.; Chilkoti, A. Nanoparticle-Film Plasmon Ruler Interrogated with Transmission Visible Spectroscopy. ACS Photonics 2014, 1 (10), 974-984. 
(56) Kristensen, A.; Yang, J. K. W.; Bozhevolnyi, S. I.; Link, S.; Nordlander, P.; Halas, N. J.; Mortensen, N. A. Plasmonic Colour Generation. Nat. Rev. Mater. 2016, 2 (1), 16088.

(57) Cormier, S.; Ding, T.; Turek, V.; Baumberg, J. J. Actuating Single Nano-Oscillators with Light. Adv. Opt. Mater. 2018, 6 (6), 1-5.

(58) Huang, Y.; Ma, L.; Li, J.; Zhang, Z. Nanoparticle-on-Mirror Cavity Modes for Huge and/or Tunable Plasmonic Field Enhancement. Nanotechnology 2017, 28 (10).

(59) Li, L.; Hutter, T.; Steiner, U.; Mahajan, S. Single Molecule SERS and Detection of Biomolecules with a Single Gold Nanoparticle on a Mirror Junction. Analyst 2013, 138 (16), 4574-4578.

(60) Chen, C. K.; De Castro, A. R. B.; Shen, Y. R. Surface-Enhanced Second-Harmonic Generation. Phys. Rev. Lett. 1981, 46 (2), 145-148.

(61) Hartstein, A.; Kirtley, J.; Tsang, J. Enhancement of the Infrared Absorption from Molecular Monolayers with Thin Metal Overlayers. Phys. Rev. Lett. 1980, 45 (3), 201-204.

(62) Guerrero, A. R.; Aroca, R. F. Surface-Enhanced Fluorescence with Shell-Isolated Nanoparticles (SHINEF). Angew. Chemie Int. Ed. 2011, 50 (3), 665-668.

(63) Fleischmann, M.; Hendra, P.; McQuillan, A. Raman Spectra of Pyridine Adsorbed at a Silver Electrode. Chem. Phys. Lett. 1974, 26 (2), 2-5.

(64) Jeanmaire, D.; Duyne, R. Van. Surface Raman Spectroelectrochemistry: Part I. Heterocyclic, Aromatic, and Aliphatic Amines Adsorbed on the Anodized Silver Electrode. J. Electroanal. Chem. 1977, 84, 1-20.

(65) Moskovits, M. Surface-Enhanced Spectroscopy. Rev. Mod. Phys. 1985, 57 (3), 783-826.

(66) Nie, S. Emory, S. Probing Single Molecules and Single Nanoparticles by SurfaceEnhanced Raman Scattering. Science (80-. ). 1997, 275 (5303), 1102-1106.

(67) Kneipp, K.; Wang, Y.; Kneipp, H.; Perelman, L. T.; Itzkan, I.; Dasari, R. R.; Feld, M. S. Single Molecule Detection Using Surface-Enhanced Raman Scattering ( SERS ). Phys. Rev. Lett. 1997, 78 (9), 1667-1670.

(68) Luo, S. C.; Sivashanmugan, K.; Liao, J. Der; Yao, C. K.; Peng, H. C. Nanofabricated SERS-Active Substrates for Single-Molecule to Virus Detection in Vitro: A Review. Biosens. Bioelectron. 2014, 61, 232-240.

(69) Xu, M. L.; Gao, Y.; Han, X. X.; Zhao, B. Detection of Pesticide Residues in Food Using Surface-Enhanced Raman Spectroscopy: A Review. J. Agric. Food Chem. 2017, 65 (32), $6719-6726$.

(70) Fikiet, M. A.; Khandasammy, S. R.; Mistek, E.; Ahmed, Y.; Halámková, L.; Bueno, J.; Lednev, I. K. Surface Enhanced Raman Spectroscopy: A Review of Recent Applications in Forensic Science. Spectrochim. Acta - Part A Mol. Biomol. Spectrosc. 2018, 197, 255260.

(71) Malyj, M.; Griffiths, J. E. Stokes/Anti-Stokes Raman Vibrational Temperatures: Reference Materials, Standard Lamps, and Spectrophotometric Calibrations. Appl. Spectrosc. 1983, 37 (4), 315-333.

(72) McCreery, R. L. Raman Spectroscopy for Chemical Analysis; John Wiley \& Sons, Inc.: Hoboken, NJ, USA, 2000.

(73) Guillot, N.; De, M. L.; de la Chapelle, M. L. The Electromagnetic Effect in Surface Enhanced Raman Scattering: Enhancement Optimization Using Precisely Controlled Nanostructures. J. Quant. Spectrosc. Radiat. Transf. 2012, 113 (18), 2321-2333.

(74) Nam, J. M.; Oh, J. W.; Lee, H.; Suh, Y. D. Plasmonic Nanogap-Enhanced Raman Scattering with Nanoparticles. Acc. Chem. Res. 2016, 49 (12), 2746-2755. 
(75) Markel, V.; Shalaev, V.; Zhang, P.; Huynh, W.; Tay, L.; Haslett, T.; Moskovits, M. NearField Optical Spectroscopy of Individual Surface-Plasmon Modes in Colloid Clusters. Phys. Rev. B 1999, 59 (16), 10903-10909.

(76) Moskovits, M. Persistent Misconceptions Regarding SERS. Phys. Chem. Chem. Phys. 2013, 15 (15), 5301-5311.

(77) Blackie, E. J.; Le Ru, E. C.; Etchegoin, P. G. Single-Molecule Surface-Enhanced Raman Spectroscopy of Nonresonant Molecules. J. Am. Chem. Soc. 2009, 131 (40), 1446614472.

(78) Lombardi, J. R.; Birke, R. L. A Unified View of Surface-Enhanced Raman Scattering. Acc. Chem. Res. 2009, 42 (6), 734-742.

(79) Efremov, E. V.; Ariese, F.; Gooijer, C. Achievements in Resonance Raman Spectroscopy. Review of a Technique with a Distinct Analytical Chemistry Potential. Anal. Chim. Acta 2008, 606 (2), 119-134.

(80) Le Ru, E. C.; Blackie, E.; Meyer, M.; Etchegoin, P. G.; Ru, E. C. Le. Surface Enhanced Raman Scattering Enhancement Factors: A Comprehensive Study. J. Phys. Chem. C 2007, 111 (37), 13794-13803.

(81) Brongersma, M. L.; Halas, N. J.; Nordlander, P. Plasmon-Induced Hot Carrier Science and Technology. Nat. Nanotechnol. 2015, 10 (1), 25-34.

(82) Baffou, G.; Quidant, R. Thermo-Plasmonics: Using Metallic Nanostructures as NanoSources of Heat. Laser Photonics Rev. 2013, 7 (2), 171-187.

(83) Govorov, A. O.; Richardson, H. H. Generating Heat with Metal Nanoparticles. Nano Today 2007, 2 (1), 30-38.

(84) Baffou, G.; Rigneault, H. Femtosecond-Pulsed Optical Heating of Gold Nanoparticles. Phys. Rev. B - Condens. Matter Mater. Phys. 2011, 84 (3), 1-13.

(85) Magnozzi, M.; Proietti Zaccaria, R.; Catone, D.; O’Keeffe, P.; Paladini, A.; Toschi, F.; Alabastri, A.; Canepa, M.; Bisio, F. Interband Transitions Are More Efficient Than Plasmonic Excitation in the Ultrafast Melting of Electromagnetically Coupled Au Nanoparticles. J. Phys. Chem. C 2019, 123 (27), 16943-16950.

(86) Huang, X. H.; El-Sayed, I. H.; Qian, W.; El-Sayed, M. a. Cancer Cell Imaging and Photothermal Therapy in the Near-Infrared Region by Using Gold Nanorods. J. Am. Chem. Soc. 2006, 128 (6), 2115-2120.

(87) Stern, J. M.; Stanfield, J.; Kabbani, W.; Hsieh, J. T.; Cadeddu, J. A. Selective Prostate Cancer Thermal Ablation with Laser Activated Gold Nanoshells. J. Urol. 2008, 179 (2), 748-753.

(88) Robert, H. M. L.; Kundrat, F.; Bermúdez-Ureña, E.; Rigneault, H.; Monneret, S.; Quidant, R.; Polleux, J.; Baffou, G. Light-Assisted Solvothermal Chemistry Using Plasmonic Nanoparticles. ACS Omega 2016, 1, 2-8.

(89) Boyd, D. A.; Greengard, L.; Brongersma, M.; El-Naggar, M. Y.; Goodwin, D. G. PlasmonAssisted Chemical Vapor Deposition. Nano Lett. 2006, 6 (11), 2592-2597.

(90) Neumann, O.; Urban, A. S.; Day, J.; Lal, S.; Nordlander, P.; Halas, N. J. Solar Vapor Generation Enabled by Nanoparticles. ACS Nano 2013, 7 (1), 42-49.

(91) Lee, B. J.; Park, K.; Walsh, T.; Xu, L. Radiative Heat Transfer Analysis in Plasmonic Nanofluids for Direct Solar Thermal Absorption. J. Sol. Energy Eng. 2012, 134 (2), 021009.

(92) Baffou, G.; Quidant, R.; García De Abajo, F. J. Nanoscale Control of Optical Heating in Complex Plasmonic Systems. ACS Nano 2010, 4 (2), 709-716. 
(93) Bohren, C. F. How Can a Particle Absorb More than the Light Incident on It?'. Am. J. Phys. 1983, 51 (4), 327-327.

(94) Zhu, X.; Vannahme, C.; Højlund-Nielsen, E.; Mortensen, N. A.; Kristensen, A. Plasmonic Colour Laser Printing. Nat. Nanotechnol. 2016, 11 (4), 325-329.

(95) Freestone, I.; Meeks, N.; Sax, M.; Higgitt, C. The Lycurgus Cup - A Roman Nanotechnology. Gold Bull. 2007, 40 (4), 270-277.

(96) Colomban, P. The Use of Metal Nanoparticles to Produce Yellow, Red and Iridescent Colour, from Bronze Age to Present Times in Lustre Pottery and Glass: Solid State Chemistry, Spectroscopy and Nanostructure. J. Nano Res. 2009, 8, 109-132.

(97) Michael Faraday; Faraday, M. X. The Bakerian Lecture. -Experimental Relations of Gold (and Other Metals) to Light. Philos. Trans. R. Soc. London 1857, 147 (0), 145-181.

(98) Kumar, K.; Duan, H.; Hegde, R. S.; Koh, S. C. W.; Wei, J. N.; Yang, J. K. W. Printing Colour at the Optical Diffraction Limit. Nat. Nanotechnol. 2012, 7 (9), 557-561.

(99) Roberts, A. S.; Pors, A.; Albrektsen, O.; Bozhevolnyi, S. I. Subwavelength Plasmonic Color Printing Protected for Ambient Use. Nano Lett. 2014, 14 (2), 783-787.

(100) Tan, S. J.; Zhang, L.; Zhu, D.; Goh, X. M.; Wang, Y. M.; Kumar, K.; Qiu, C.; Yang, J. K. W. Plasmonic Color Palettes for Photorealistic Printing with Aluminum Nanostructures. Nano Lett. 2014, 14 (7), 4023-4029.

(101) Franklin, D.; Chen, Y.; Vazquez-Guardado, A.; Modak, S.; Boroumand, J.; Xu, D.; Wu, S. T.; Chanda, D. Polarization-Independent Actively Tunable Colour Generation on Imprinted Plasmonic Surfaces. Nat. Commun. 2015, 6, 1-8.

(102) Clausen, J. S.; Højlund-Nielsen, E.; Christiansen, A. B.; Yazdi, S.; Grajower, M.; Taha, H.; Levy, U.; Kristensen, A.; Mortensen, N. A. Plasmonic Metasurfaces for Coloration of Plastic Consumer Products. Nano Lett. 2014, 14 (8), 4499-4504.

(103) Miyata, M.; Hatada, H.; Takahara, J. Full-Color Subwavelength Printing with GapPlasmonic Optical Antennas. Nano Lett. 2016, 16 (5), 3166-3172.

(104) Rezaei, S. D.; Hong Ng, R. J.; Dong, Z.; Ho, J.; Koay, E. H. H.; Ramakrishna, S.; Yang, J. K. W. Wide-Gamut Plasmonic Color Palettes with Constant Subwavelength Resolution. ACS Nano 2019, 13 (3), 3580-3588.

(105) Mukherjee, S.; Libisch, F.; Large, N.; Neumann, O.; Brown, L. V.; Cheng, J.; Lassiter, J. B.; Carter, E. A.; Nordlander, P.; Halas, N. J. Hot Electrons Do the Impossible: PlasmonInduced Dissociation of H 2 on Au. Nano Lett. 2013, 13 (1), 240-247.

(106) Narang, P.; Sundararaman, R.; Atwater, H. A. Plasmonic Hot Carrier Dynamics in SolidState and Chemical Systems for Energy Conversion. Nanophotonics 2016, 5 (1), 96-111.

(107) Hartland, G. V.; Besteiro, L. V.; Johns, P.; Govorov, A. O. What's so Hot about Electrons in Metal Nanoparticles? ACS Energy Lett. 2017, 2 (7), 1641-1653.

(108) Kong, X. T.; Wang, Z.; Govorov, A. O. Plasmonic Nanostars with Hot Spots for Efficient Generation of Hot Electrons under Solar Illumination. Adv. Opt. Mater. 2017, 5 (15), 1-10.

(109) Zheng, B. Y.; Zhao, H.; Manjavacas, A.; McClain, M.; Nordlander, P.; Halas, N. J. Distinguishing between Plasmon-Induced and Photoexcited Carriers in a Device Geometry. Nat. Commun. 2015, 6, 1-7.

(110) Panayotov, D. A.; Frenkel, A. I.; Morris, J. R. Catalysis and Photocatalysis by Nanoscale Au/TiO2: Perspectives for Renewable Energy. ACS Energy Lett. 2017, 2 (5), 1223-1231.

(111) Sun, Y. Controlled Synthesis of Colloidal Silver Nanoparticles in Organic Solutions: Empirical Rules for Nucleation Engineering. Chem. Soc. Rev. 2013, 42 (7), 2497-2511.

(112) LaMer, V. K.; Dinegar, R. H. Theory, Production and Mechanism of Formation of 
Monodispersed Hydrosols. J. Am. Chem. Soc. 1950, 72 (8), 4847-4854.

(113) Xia, Y.; Xiong, Y.; Lim, B.; Skrabalak, S. E. Shape-Controlled Synthesis of Metal Nanocrystals: Simple Chemistry Meets Complex Physics? Angew. Chemie Int. Ed. 2009, 48 (1), 60-103.

(114) Wang, F.; Richards, V. N.; Shields, S. P.; Buhro, W. E. Kinetics and Mechanisms of Aggregative Nanocrystal Growth. Chem. Mater. 2014, 26 (1), 5-21.

(115) Xiong, Y.; Xia, Y. Shape-Controlled Synthesis of Metal Nanostructures: The Case of Palladium. Adv. Mater. 2007, 19 (20), 3385-3391.

(116) Chen, J.; Lim, B.; Lee, E. P.; Xia, Y. Shape-Controlled Synthesis of Platinum Nanocrystals for Catalytic and Electrocatalytic Applications. Nano Today 2009, 4 (1), 81-95.

(117) Xia, X.; Zeng, J.; Zhang, Q.; Moran, C. H.; Xia, Y. Recent Developments in ShapeControlled Synthesis of Silver Nanocrystals. J. Phys. Chem. C 2012, 116 (41), 2164721656 .

(118) Rycenga, M.; Cobley, C. M.; Zeng, J.; Li, W.; Moran, C. H.; Zhang, Q.; Qin, D.; Xia, Y. Controlling the Synthesis and Assembly of Silver Nanostructures for Plasmonic Applications. Chem. Rev. 2011, 111 (6), 3669-3712.

(119) Siekkinen, A. R.; McLellan, J. M.; Chen, J.; Xia, Y. Rapid Synthesis of Small Silver Nanocubes by Mediating Polyol Reduction with a Trace Amount of Sodium Sulfide or Sodium Hydrosulfide. Chem. Phys. Lett. 2006, 432 (4-6), 491-496.

(120) Xiong, Y.; Washio, I.; Chen, J.; Cai, H.; Li, Z.-Y.; Xia, Y. Poly(Vinyl Pyrrolidone): A Dual Functional Reductant and Stabilizer for the Facile Synthesis of Noble Metal Nanoplates in Aqueous Solutions. Langmuir 2006, 22 (20), 8563-8570.

(121) Wiley, B.; Herricks, T.; Sun, Y.; Xia, Y. Polyol Synthesis of Silver Nanoparticles: Use of Chloride and Oxygen to Promote the Formation of Single-Crystal, Truncated Cubes and Tetrahedrons. Nano Lett. 2004, 4 (9), 1733-1739.

(122) Sun, Y.; Xia, Y. Shape-Controlled Synthesis of Gold and Silver Nanoparticles. Science (80-. ). 2002, 298 (5601), 2176-2179.

(123) Skrabalak, S. E.; Au, L.; Li, X.; Xia, Y. Facile Synthesis of Ag Nanocubes and Au Nanocages. Nat. Protoc. 2007, 2 (9), 2182-2190.

(124) Skrabalak, S. E.; Wiley, B. J.; Kim, M.; Formo, E. V; Xia, Y. On the Polyol Synthesis of Silver Nanostructures: Glycolaldehyde as a Reducing Agent. Nano Lett. 2008, 8 (7), 2077-2081.

(125) Zhou, Y.; Saidi, W. A.; Fichthorn, K. A. Comparison of the Binding of Polyvinylpyrrolidone and Polyethylene Oxide to Ag Surfaces: Elements of a Successful Structure-Directing Agent. J. Phys. Chem. C 2013, 117 (21), 11444-11448.

(126) Qi, X.; Balankura, T.; Zhou, Y.; Fichthorn, K. A. How Structure-Directing Agents Control Nanocrystal Shape: Polyvinylpyrrolidone-Mediated Growth of Ag Nanocubes. Nano Lett. 2015, 15 (11), 7711-7717.

(127) Zhang, Q.; Li, W.; Moran, C.; Zeng, J.; Chen, J.; Wen, L.-P.; Xia, Y. Seed-Mediated Synthesis of Ag Nanocubes with Controllable Edge Lengths in the Range of 30-200 Nm and Comparison of Their Optical Properties. J. Am. Chem. Soc. 2010, 132 (32), 1137211378.

(128) Xia, H.; Su, G.; Wang, D. Size-Dependent Electrostatic Chain Growth of PH-Sensitive Hairy Nanoparticles. Angew. Chemie - Int. Ed. 2013, 52 (13), 3726-3730.

(129) Toma, M.; Toma, K.; Michioka, K.; Ikezoe, Y.; Obara, D.; Okamoto, K.; Tamada, K. Collective Plasmon Modes Excited on a Silver Nanoparticle 2D Crystalline Sheet. Phys. Chem. Chem. Phys. 2011, 13 (16), 7459-7466. 
(130) Henzie, J.; Grünwald, M.; Widmer-Cooper, A.; Geissler, P. L.; Yang, P. Self-Assembly of Uniform Polyhedral Silver Nanocrystals into Densest Packings and Exotic Superlattices. Nat. Mater. 2011, 11 (2), 131-137.

(131) Gong, J.; Li, G.; Tang, Z. Self-Assembly of Noble Metal Nanocrystals: Fabrication, Optical Property, and Application. Nano Today 2012, 7 (6), 564-585.

(132) Choueiri, R. M.; Klinkova, A.; Thérien-Aubin, H.; Rubinstein, M.; Kumacheva, E. Structural Transitions in Nanoparticle Assemblies Governed by Competing Nanoscale Forces. J. Am. Chem. Soc. 2013, 135 (28), 10262-10265.

(133) Edel, J. B. J.; Kornyshev, A. A. a; Urbakh, M. Self-Assembly of Nanoparticle Arrays for Use as Mirrors, Sensors, and Antennas. ACS Nano 2013, 7 (11), 9526-9532.

(134) Acharya, S.; Hill, J. P.; Ariga, K. Soft Langmuir-Blodgett Technique for Hard Nanomaterials. Adv. Mater. 2009, 21 (29), 2959-2981.

(135) Shaw, M.; Fairchild, M. Evaluating the 1931 CIE Color-Matching Functions. Color Res. Appl. 2002, 27 (5), 316-329.

(136) Fairman, H. S.; Brill, M. H.; Hemmendinger, H. How the CIE 1931 Color-Matching Functions Were Derived from Wright-Guild Data. Color Res. Appl. 1997, 22 (1), 11-23. 


\section{Chapter 2: Numerical Modelling of Hybrid Plasmonic Modes in Anisotropic Systems}

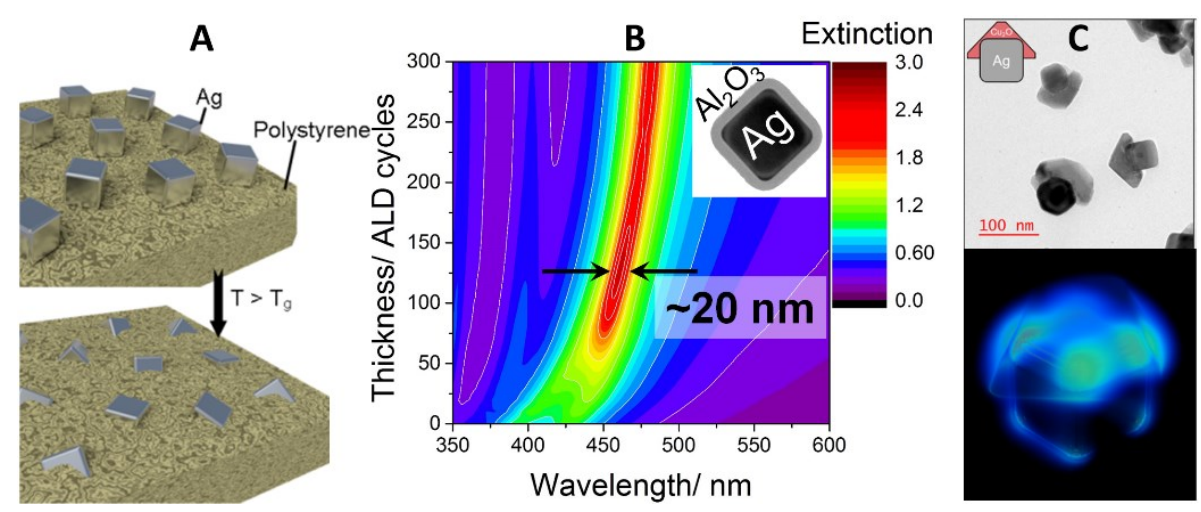

\section{Abstract}

Hybridized plasmonic modes of silver nanocubes are a unique phenomenon which produce spectrally and spatially separated dipolar (D) and quadrupolar (Q) modes. The local dielectric environment can be used to heavily influence the position and spatial distribution of these modes and this can be done so selectively. The following work presents 3 separate instances where the local dielectric environment around the nanocube is altered and the hybrid plasmon modes produce distinct effects with unique functions. In the first section the nanocube is embedded into its dielectric support, and the unique spectral and spatial characteristics of the plasmon resonance can precisely monitor the distance the nanocube has travelled. In the second portion the over-coating of the nanocubes with a thin dielectric film produces a uniquely sharp localized surface plasmon resonance. In the third example, hybrid core@half-shell asymmetric particles are produced with unique optical properties. In all three cases numerical modelling by the finite-difference timedomain technique was an invaluable tool in predicting the spectral characteristics of the system and determining the spatial distribution of the modes.

Bottomley, A.; Prezgot, D.; Coyle, J. P.; lanoul, A. Dynamics of Nanocubes Embedding into Polymer Films Investigated via Spatially Resolved Plasmon Modes. Nanoscale 2016, 8 (21), 11168-11176.

Prezgot, D.; Bottomley, A.; Coyle, J. P.; lanoul, A. Unusually Sharp Localized Surface Plasmon Resonance in Supported Silver Nanocrystals with a Thin Dielectric Coating. J. Phys. Chem. Lett. 2017, 8 (22), 5555-5558.

Gale-Mouldey, A.; Jorgenson, E.; Coyle J. P.; Prezgot, D.; lanoul, A.; Hybridized plasmon resonances in core/half-shell cuprous oxide nanoparticles., J. Mater Chem. C., 2020, Advance Article 


\section{Dynamics of nanocubes embedding into polymer films investigated via spatially resolved plasmon modes}

\subsection{Part A: Introduction}

The use of noble metal nanoparticles is extensive due to their ability to support localized surface plasmons yielding intense optical properties and extremely high sensitivities to material, geometry, and local environment. ${ }^{1}$ This enables them to be effective as nanorulers for subdiffraction measurements that can be taken using simple far-field observations achieving spatial resolutions on the nanometer and in some cases angstrom scale. ${ }^{2-6}$ The high sensitivity to changes in local environment surrounding plasmonic nanoparticles can be used to investigate phenomena such the glass transition of various polymer films allowing direct investigations into the thermodynamics of polymer interfaces. ${ }^{7-9}$ These types of investigations are of importance as polymers are a common choice for solid supported nanomaterials.

Precise control over the relative orientation and distances of nanocrystals is critical in nanoscale engineering and design when the geometry of individual components in various nanoarchitectures defines their useful properties. In this respect, the ability to continuously monitor nanoscale assembly processes over large distances with high precision remains desirable yet challenging. To address these needs a wide variety of nanorulers utilizing various interactions and properties of plasmonic nanomaterials have been explored. This includes rulers based on coupling interactions between adjacent particles, ${ }^{4,10-12}$ Fano interferences as a result of the interaction, ${ }^{10,13}$ and particle coupling with metal surfaces. ${ }^{12,14,26}$ Another common approach to plasmonic based nanorulers utilizes the relationship between a plasmon and an emitter of some kind, including distance dependent surface enhanced Raman spectroscopy, ${ }^{14}$ nanometal surface energy transfer interactions, ${ }^{3,15}$ and plasmophore surface enhanced fluorescence. ${ }^{16}$ The resolution, range, sensitivity, and ease of fabrication of nanorulers varies considerably, in some cases yielding angstrom resolution for interparticle/substrate coupling bands, while other systems can attain up to $100 \mathrm{~nm}$ in dynamic range such as the plasmophore based systems. A common element of the nanorulers described above is the interaction of a plasmonic particle with a specific nearby object, often this is another metal particle, surface, or an emitter such as a fluorophore. Alternatively, some 
nanorulers are simply based on the change in refractive index of their surrounding environment. These simple nanorulers have been shown to accurately determine the thickness of dielectric spacers up to $30 \mathrm{~nm} \cdot{ }^{17}$

Of particular interest, due to their ability to support strong hybridized modes, ${ }^{18}$ silver nanocubes are an excellent candidate for use as a nanoruler and optical reporter in composite materials, as individual nanocubes are highly sensitive to anisotropy in their local envrionment. ${ }^{19}$ Thus, it is possible to gain significant control over the optical properties of silver nanocubes, while also gaining insight into the position, orientation, and degree of anisotropy in their local refractive index at any time. The spatially separated modes that occur when silver nanocubes are placed in an anisotropic environment allow for their spectral response to be tuned independently for a small range in the visible spectrum. This level of control and information feedback about the conditions surrounding the nanocrystals makes this platform an effective tool for the production of composite materials with specific properties.

The present work investigates of hybrid plasmonic modes generated by anisotropy of the surrounding environment can yield significant control over the spectral signature of silver nanocubes. In addition to spectral control the response of these hybrid modes to their changing environment allows them to be used as a set of three complementary nanorulers giving a combined dynamic range of $\sim 60 \mathrm{~nm}$. The use of finite-difference time-domain modelling was used to examine the spectral evolution of the system and examine the response provided by the complex spatial reorientation of nanocrystals and their hybrid modes.

\subsection{Results and Discussion}

\subsubsection{Spectral Characteristics of Embedding Nanocubes into polystyrene.}

Supported silver nanocubes show very characteristic extinction spectra that depend on the size of the cubes and the dielectric properties of the support. ${ }^{20,21}$ There are two hybrid peaks present in the spectra assigned as a dipolar and quadrupolar surface plasmon resonances.

The experimentally produced nanocubes in this work had a length of $62 \pm 11 \mathrm{~nm}$ (Figure A.1). The size distribution is not particularly narrow, however the size dependence for the position of the quadrupolar resonance is relatively weak. ${ }^{22}$ The corresponding UV-vis extinction spectrum 
shows characteristic sharp signatures with relatively narrow half widths when on a substrate. The dipole (D) resonance for the nanocubes while in solution is at $481 \mathrm{~nm}$ with the measured full width at half maximum (FWHM) around $50 \mathrm{~nm}$ (Figure 2.1A). Other higher order resonances present in the solution spectrum are at 393 and $346 \mathrm{~nm}$. The calculated E-field distribution from finitedifference time-domain (FDTD) modelling corresponding to these modes is shown as insets in Figure $2.1 \mathrm{~A}$ and appears to be symmetrical.
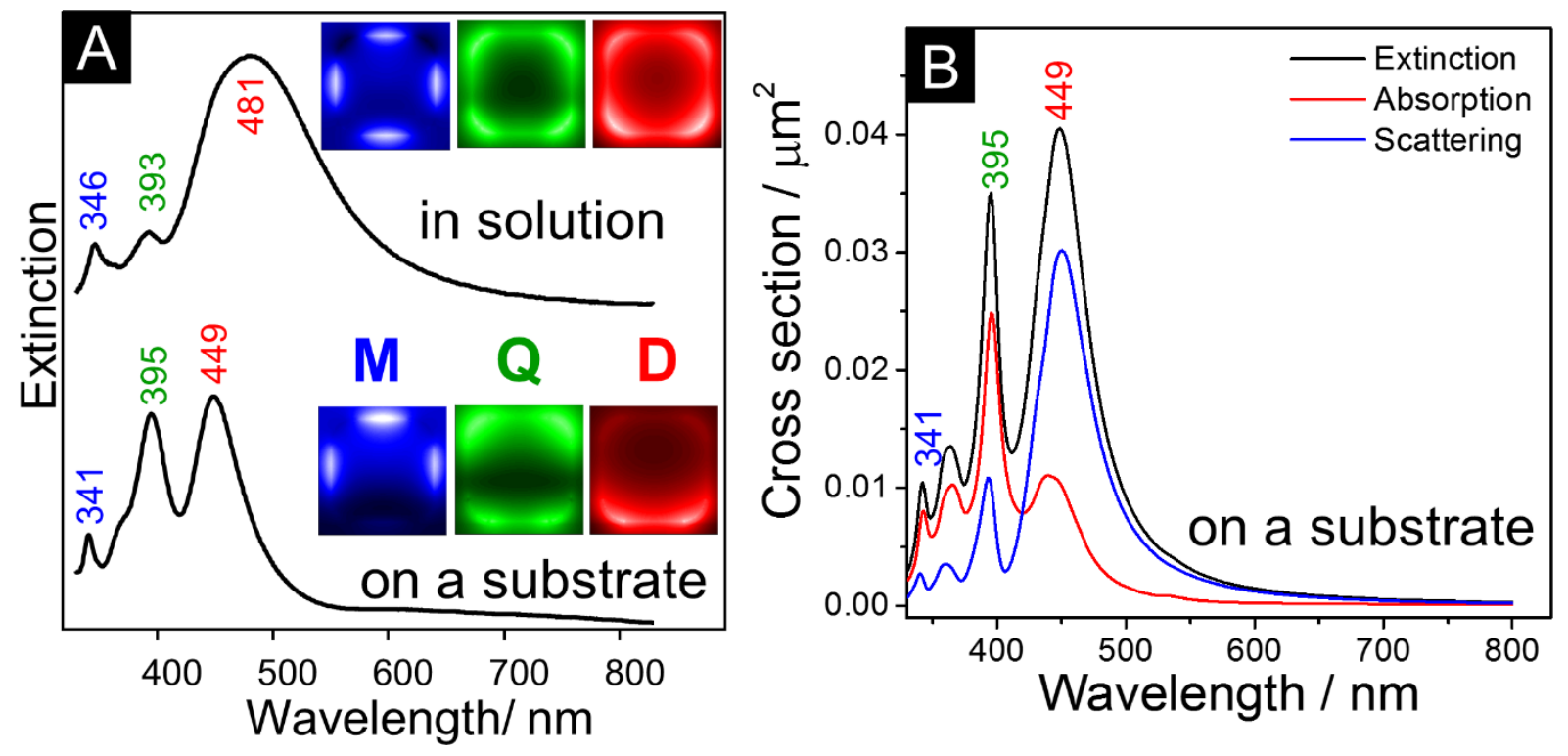

Figure 2.1. A) UV-visible extinction spectra of a nanocube sample in ethanol (top) and on a polystyrene substrate in air (bottom). E-field distributions for the dipolar (D), quadrupolar (Q), and multipolar (M) resonances are shown for both environments. B) FDTD model of the extinction, absorbance and scattering cross-sections of a nanocube on polystyrene. The peak positions are in agreement with the experiment.

When supported by a thin polystyrene (PS) film the cubes exhibit an even narrower hybrid dipolar resonance (D) at $449 \mathrm{~nm}(\mathrm{FWHM} \sim 40 \mathrm{~nm})$, a hybrid quadrupolar resonance (Q) at $395 \mathrm{~nm}$ (FWHM $30 \mathrm{~nm}$ ), and a multipolar resonance (M) at $341 \mathrm{~nm}(14 \mathrm{~nm})$ (Figure 2.1A). Associated Efields are shown in Figure 2.1A extinction, scattering and absorbance cross sections from FDTD are shown in Figure 2.1B. The model consisted of a $62 \mathrm{~nm}$ nanocube with a corner roundness of $12 \mathrm{~nm}$, and a $2 \mathrm{~nm}$ layer of PVP. The modelled peak position is in excellent agreement with the ensemble measurement, with narrower peaks (FWHM $\sim 30 \mathrm{~nm}, 20 \mathrm{~nm}$ for $D$ and $Q$ modes respectively). Peaks are expected to be wider for the ensemble measurement due to the polydispersity in size of the experimentally produced sample. 
The E-field distribution maps (Figure 2.1A inset) show that the E-field associated with the hybrid dipolar resonance is primarily located at the bottom part of the cube, quadrupolar at the top, and multipolar resonances have their significant component associated with the sides of the cube. Assignment and spatial distribution of the resonances have been studied theoretically and experimentally, for example with the help of electron energy loss spectroscopy23 and cathodoluminescence.24 The spatially resolved nature of plasmonic modes as well as small FWHMs enables precise monitoring of the dynamics and changing local environment around the nanocubes as they embed into their supporting polymer.

At room temperature PS exists in the glassy state and can therefore act as a dielectric substrate smooth enough to support nanocube monolayers. Bulk PS experiences a glass transition at $\sim 100{ }^{\circ} \mathrm{C} .25$ While the transition temperature depends factors such as the chain length and tacticity, a significant depression in the $\mathrm{Tg}$ to $\sim 75^{\circ} \mathrm{C}$ occurs for ultra-thin films. 26 Extinction spectra of the nanocube monolayers are allowed to evolve over time when the system is kept above the supporting PS glass transition temperature at $112 \pm 1^{\circ} \mathrm{C}$ (Figure 2.2). The observed changes are not a result from nanocube transformation, such as rounding or oxidation,22 but from their response to being embedded into the PS film. Exclusion of thermally induced damage was addressed by comparing the PS supported sample to a glass supported sample. The glass supported cubes, heated alongside the PS supported cubes showed no changes in their extinction spectrum at the end of the experiment. Therefore, the observed spectroscopic evolution is associated with the nanocrystals embedding into the polystyrene film exclusively.

The spectroscopic changes that occur with time can be separated into for stages shown in Figure 2.2. The first stage is characterized by a very strong red-shift of the $D$ resonance from the original $449 \mathrm{~nm}$ to $480 \mathrm{~nm}$. Both the position and intensity of the $\mathrm{Q}$ resonance remain almost invariant. In the M resonance region, a peak around $350 \mathrm{~nm}$ is developed in addition to the original peak at $341 \mathrm{~nm}$. Topology measurements by atomic force microscopy show the average height of the nanocubes within the monolayer decreases from $75 \mathrm{~nm}$ to $55 \mathrm{~nm}$ while retaining their orientation and spatial arrangement. 
During the second stage both $D$ and $Q$ resonances slightly red-shift. At the same time, the $M$ resonance shows a two state-like transition between the peaks at $\sim 341 \mathrm{~nm}$ and $\sim 350 \mathrm{~nm}$. The average height of the exposed nanocubes decreases to $\sim 45 \mathrm{~nm}$, occurring between 2 and 7 minutes of heating.
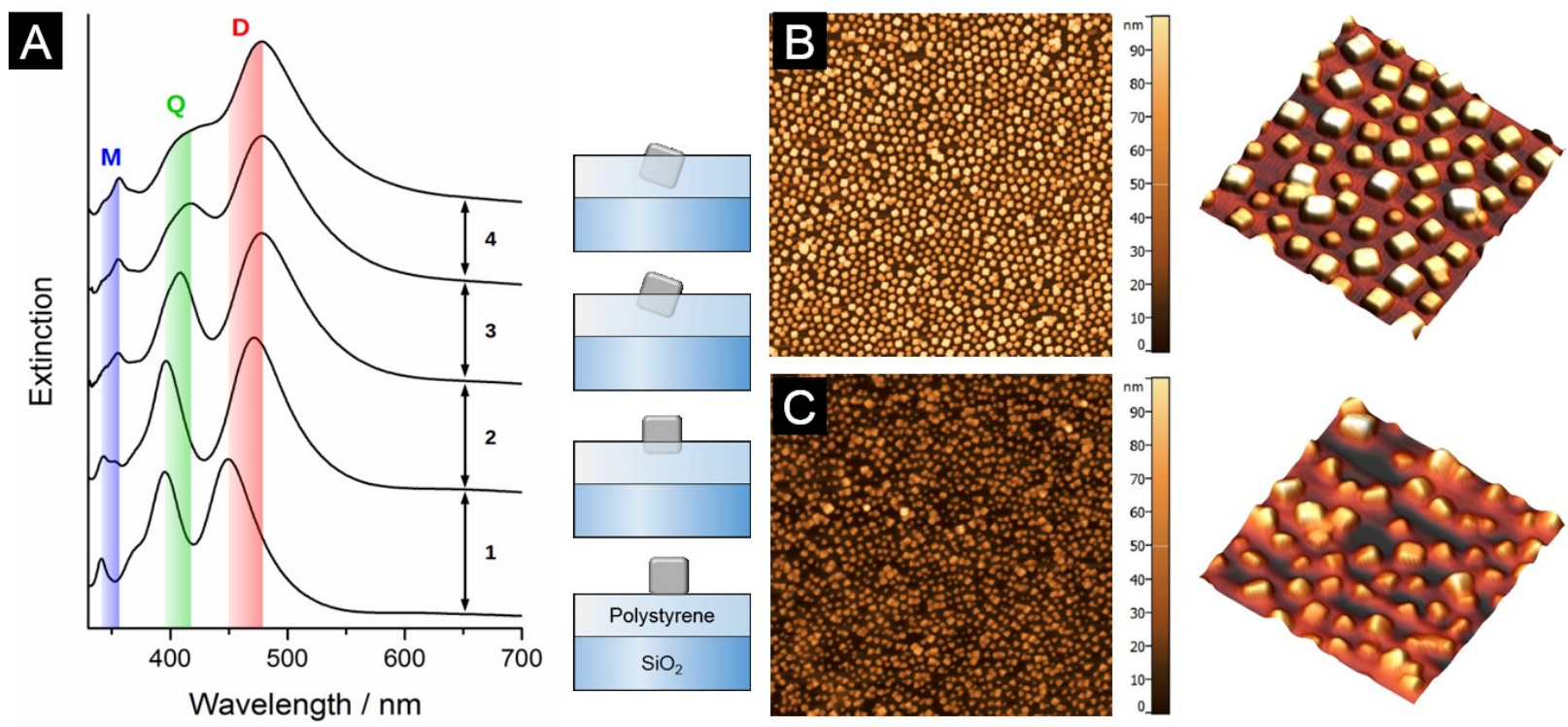

Figure 2.2 A) Spectral evolution of the embedment process occurs at 4 distinct stages: 1) An initial shift of the $D$ mode and a transition of the $M$ mode followed by 2) a shift in both the $D$ and $Q$ mode 3) shift of only the $Q$ mode 4) merging of the $D$ and $Q$ modes. B,C) AFM measurements of the nanocubes before and after the embedment process.

During the third stage, the $D$ resonance reaches a max at $485 \mathrm{~nm}, \mathrm{Q}$ shows the greatest redshift to $\sim 414 \mathrm{~nm}$, the resonance at 340 disappears, leaving only a $350 \mathrm{~nm}$ multipolar resonance in the region. layer. The average height decreases below $30 \mathrm{~nm}$, which is roughly half of the nanocube size. By this point nearly all of the cubes have experienced some type of tilting motion

Finally, during the fourth stage the $\mathrm{D}$ and $\mathrm{M}$ resonances remain unchanged. At the same time, the $Q$ resonance becomes obscured by the overlap with the $D$ mode. The final stage in this experiment corresponds to almost complete submergence of the particles. The average height decreases to less than $25 \mathrm{~nm}$ with the majority of cubes having only a single corner exposed (Figure $2.2 \mathrm{C})$.

The spectral changes can be reasonably well explained by the process of incorporation of nanocubes into the PS film upon heating observed by AFM (Figure 2.2B). As a result of incorporation, the environment surrounding the nanocubes becomes more isotropic and the 
spectrum moves towards that of nanocubes in a high refractive index solution as hybridization of the plasmonic modes is reduced.

\subsubsection{Modelling extinction of partially embedded cubes}

To investigate the process in more detail the finite-difference time-domain (FDTD) method was used to model the extinction spectra of a nanocube embedded iteratively in $5 \mathrm{~nm}$ steps into a polystyrene film (Figure 2.3). The nanocube's orientation as well as the light source's angle of incidence were kept normal to the substrate. As the nanocube is lowered into the substrate a rapid shift in the $D$ mode is initially observed while the $Q$ remains relatively insensitive; the $D$ mode shifts $\sim 60 \mathrm{~nm}$ while the $\mathrm{Q}$ only shifts $\sim 5 \mathrm{~nm}$ at $30 \mathrm{~nm}$ of immersion. As the cube is further immersed in the substrate the sensitivity of the $D$ mode gradually diminishes as the $Q$ mode's sensitivity increases until it reaches a maximum at $55 \mathrm{~nm}$ of immersion. This results in a red-shift of the $\mathrm{Q}$ mode by $\sim 50 \mathrm{~nm}$ between $30 \mathrm{~nm}$ and $60 \mathrm{~nm}$ of immersion, while the $D$ mode only shifts $15 \mathrm{~nm}$ (Figure 2.3).

This model reliably follows the trends observed experimentally. ${ }^{27}$ When comparing sensitivities derived from the simulations to experimentally observed values we find the $D$ mode is shown to have a maximum sensitivity of $4.0 \mathrm{~nm} / \mathrm{nm}$ as opposed to $2.1 \mathrm{~nm} / \mathrm{nm}$ while the $Q$ reaches a maximum of $1.8 \mathrm{~nm} / \mathrm{nm}$ as opposed to $1.2 \mathrm{~nm} / \mathrm{nm}$. The increased sensitivity observed in the model is a consequence it describing an idealized scenario involving only a single nanocube. The experimental measurements are performed on an ensemble of nanocubes which introduce a degree of polydispersity in terms of size, depth of immersion, and orientation, all of which contribute to band-broadening and consequently a reduction in the sensitivity when compared to the single particle model. 

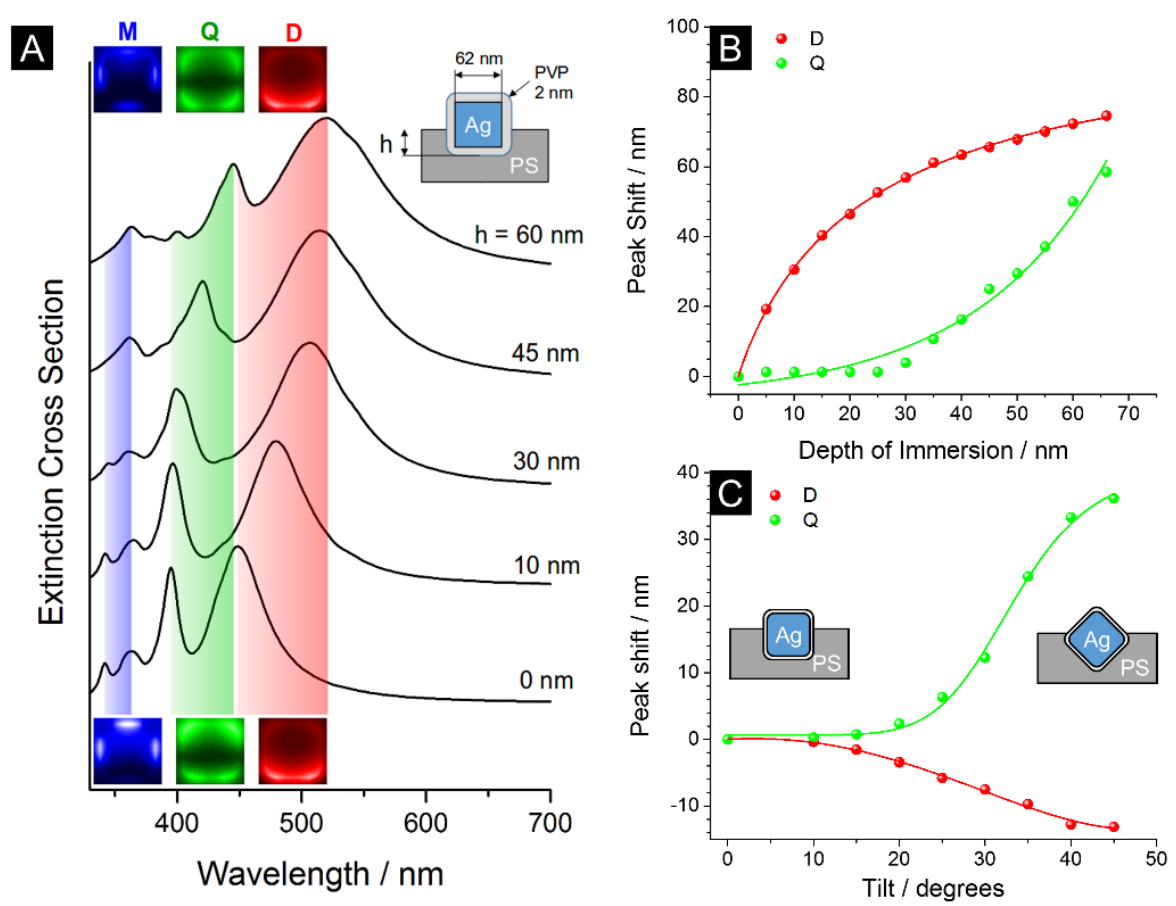

Figure 2.3 A) FDTD calculated extinction spectra of a silver nanocube in polystyrene film for different embedding depths. E-Field distributions (imbedded) are given for the initial and final states of the simulation. The red, green, and blue bands indicate the overall shift for $D, Q$, and $M$ modes respectively. B) Peak shifts from FDTD modelling displaying peak shift versus depth of immersion for a normal oriented $62 \mathrm{~nm}$ cube imbedding into polystyrene. C) Relative peak shift against angle of tilt about a single axis for a $62 \mathrm{~nm}$ cube held at a constant depth of $40 \mathrm{~nm}$ in polystyrene. Very little change is observed at a small angles $\left(<15^{\circ}\right)$, but increasing the angle of tilt results in a small blue shift of the $D$ mode and a rapid red shift of the $Q$ mode.

While a general agreement between the model and experimental results was obtained, it should be noted that the model begins to diverge from the experimental results after the cube is immersed at a depth greater than half its edge length. While almost no further peak shift of the $D$ mode was observed after the cubes are half immersed experimentally (Figure 2.2), the model predicts a further peak shift of $20 \mathrm{~nm}$ between during the second half of the experiment (Figure 2.3B). The $Q$ mode also shifts relatively faster experimentally than predicted by the model; the predicted point of maximum sensitivity of the $Q$ mode is after the nanocubes have been immersed $55 \mathrm{~nm}$, while we observe this point at $36 \mathrm{~nm}$ of immersion in the experiment. A shift to lower energy for the $\mathrm{M}$ mode is predicted by the model occurring after the cube has been half- immersed, while the actual transition observed experimentally occurs at a much earlier stage. This discrepancy may be due to the fact that the error tends to be significant near the interband transition at $3.8 \mathrm{eV}(326$ $\mathrm{nm}$ ) in the imaginary component of the dielectric function by which silver is described in the model. ${ }^{28}$ Due to the uncertainty in the origin of this discrepancy it is difficult to discuss the modelled results 
of the $\mathrm{M}$ mode in detail. The divergence between the model and experimental results primarily correspond with our third stage of experimental observations where tilting of the nanocubes becomes significant. The model described thus far has consisted of a nanocube orientated normal to the substrate. It therefore becomes necessary to examine the effect of nanocube orientation on the optical properties of the particle in order to consolidate the predicted and experimental results. FDTD simulations were utilized to independently examine the effect of nanocube orientation on their extinction spectra using an unpolarized light source orientated perpendicular to the substrate, which we define as the $z$ axis. A series of simulations were performed on cube immersed $40 \mathrm{~nm}$ into polystyrene which was rotated between $0^{\circ}$ and $45^{\circ}$ about a single axis through its center. Experimentally the cubes are free to rotate along three degrees of freedom in terms of $\mathrm{x}, \mathrm{y}$ and $\mathrm{z}$ axes. Individual rotation about the $\mathrm{x}$ and $\mathrm{y}$ axes are degenerate in terms of spectral response while rotation about the $z$ axis is unobservable as all orientations are present at the initial and final states of the experiment. We observe cubes having rotated about both $\mathrm{x}$ and $\mathrm{y}$ axes at a variety of angles after 30 minutes of heating (Figure 2.2C), with the majority having rotated to large angles $\left(>25^{\circ}\right)$ on at least one of the two axes. Figure 2.3C describes the peak shift of the $D$ and $Q$ modes as the cube is rotated about a single axis. A rotation of less than $15^{\circ}$ results in very little spectral response, but further rotation results in a blue shift of the $D$ mode by $13 \mathrm{~nm}$ and a red shift of the $Q$ mode up to $36 \mathrm{~nm}$ at $45^{\circ}$. By contrasting these spectral shifts against those associated with the normal-oriented cube model we illustrate that the experimental shifts in the final stages of the experiment arise from the combination of the nanocube immersion and change in orientation which occur simultaneously. While a small $(\sim 15 \mathrm{~nm})$ red shift of the $D$ mode is predicted for the last $20 \mathrm{~nm}$ of immersion of a normal-oriented nanocube (Figure 2.3A), the change in orientation of the nanocube results in a comparable blue shift. The competition between these phenomena leads to the observation of the apparent saturation of the shift of the D mode. Meanwhile, the red shift of the $Q$ mode associated with the change in orientation of the nanocube accumulates with the red shift associated with further immersion of the nanocube. The combined effect leads to a more rapid shift the $Q$ mode than would be observed if the nanocube did not change orientation. 


\subsubsection{Subfacial Sensitivity of Hybridized Modes}

As long as there is refractive index contrast present in a system spatially resolved plasmon modes can be used to track the changes in their surroundings. The initial optical signature and spectral evolution of the system as particles embed themselves into the polymer layer are dependent on the refractive index of the surrounding environment above and below the nanocrystal. As the system evolves, it moves from a strongly hybridized regime resulting from high anisotropy to a more isotropic system that results in less hybridization as the cubes embed themselves into the polymer layer. However, if the polymer layer is chosen such that it is thin enough that cubes can nearly reach the bottom of it, and the film is situated on a substrate of relatively high refractive index it becomes possible to push the system back in the direction of hybridization by inducing anisotropy within the material. This scenario was experimentally explored by using a thin polystyrene film $(\sim 50 \mathrm{~nm})$ on top of a high refractive index substrate of titanium oxide (Figure A.2). As a result of the increased refractive index of the substrate, the D mode does not saturate before the cubes stop embedding. As the relative refractive indices of both the substrate and superstrate are higher than the initial setup at time zero, all modes are red shifted but maintain a degree of hybridization. This system adds an extra dimension to the nanoruler, extending its dynamic range. Similar improvements can be made by choosing a higher refractive index polymer, again increasing the sensitivity and dynamic range of the system. High- $n$ polymers such as polyphosphazenes and polyphosphonates provide an attractive alternative to polystyrene due to their high refractive index, up to 1.75 compared to polystyrene's $n$ of 1.60 at $550 \mathrm{~nm}$ as well as a similar $\mathrm{T}_{\mathrm{g}}$ and processability. ${ }^{29}$ FDTD modelling was employed to compare the refractive index sensitivity of the nanoruler on a polymer with $n=1.75$ against the polystyrene model (Figure 2.4). An improvement in the maximum sensitivity of both the $D$ and $Q$ mode was observed. The $D$ mode's initial sensitivity increases from 4.0 to $5.0 \mathrm{~nm} / \mathrm{nm}$, while the $Q$ mode's maximum sensitivity increases from 1.8 to $2.2 \mathrm{~nm} / \mathrm{nm}$ at $50 \mathrm{~nm}$ of immersion. With alternative material choices of both the substrate and polymer film; provided the process of incorporation remains energetically favorable, it becomes possible to further improve the sensitivity and dynamic range for this type of nanoruler. 

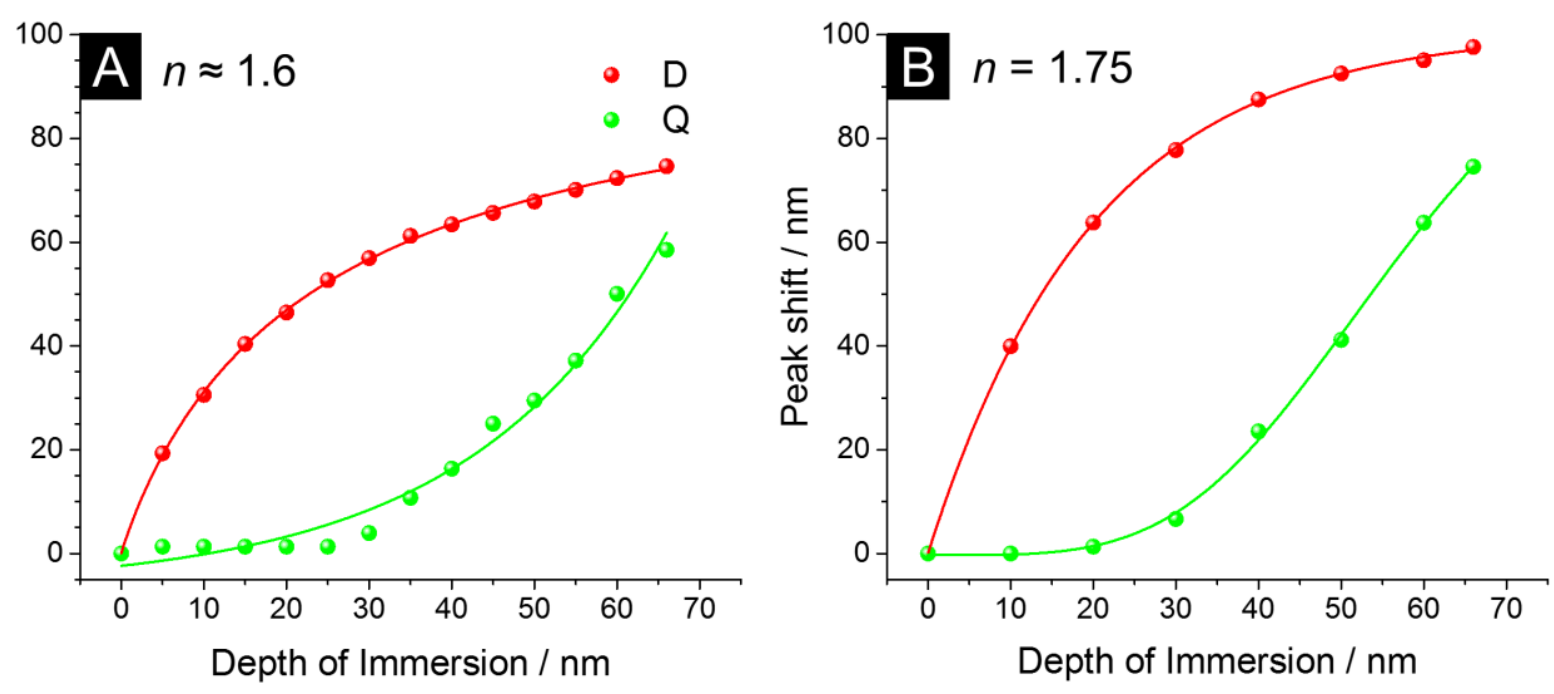

Figure 2.4 Comparison of peak sensitivity in models of nanocube embedment using A) Polystyrene, by $n(\lambda)$ where on average $n \approx 1.6$, and $\mathbf{B}$ ) a representation of a high- $n$ polymer, with $n=1.75$. The high- $n$ produces more sensitive peak shifts.

\subsection{Conclusion}

A nanocomposite comprised of silver nanocube monolayers on polystyrene thin films was fabricated as a platform for the precise control and observation of nanoparticles embedding into a polymer. We have also determined that once a system is carefully calibrated it becomes possible to know the average distance and orientation of the nanocrystals embedded into the polymer using simple far field optical measurements alone. This type of anisotropic nanoruler is based on plasmon hybridization induced by proximity to a dielectric substrate and utilizes the combined refractive index sensitivity of the spatially resolved plasmon modes. The use of FDTD modelling was able to correlate the sensitivity of the plasmon modes with their spatial distribution, predict the upper limit of the sensitivity of the system, and allowed for independent investigation of how tilting of the nanocubes effects the spectral response of the system. These determinations were important to the final characterization of the system and helped demonstrate how hybrid plasmon modes can be advantageous in use as a nanoruler. 


\section{Unusually sharp localized surface plasmon resonances in supported silver nanocrystals with a thin dielectric coating}

\subsection{Part B: Introduction}

High quality of optical resonances in plasmonic nanoparticles is essential for efficient functional use of light energy. By adjusting the local environment of the nanoparticle, the resonances can be tailored. ${ }^{30,31}$ More precise control is observed for supported nanoparticles where environment becomes anisotropic, ${ }^{18,32}$ and several plasmon modes emerge as degeneracy is removed. The effect is particularly strong in cases when the contact area between the plasmonic nanoparticle and the substrate is large compared to the total nanoparticle surface area. ${ }^{18}$ For example, the extinction spectra of silver nanocubes undergo dramatic alteration when supported by a dielectric substrate: instead of a single dipolar localized surface plasmon resonance (LSPR) for suspended particles, two separate peaks appear as a result of the dipolar (D) and quadrupolar (Q) modes mixing. 18,20,33 The new hybrid resonances correspond to charge oscillations close to and away from the substrate and can be tuned independently. This substrate-induced anisotropy can be beneficial for many plasmon-based applications, including near-field (SERS) ${ }^{34}$ or far-field (light extinction) spectroscopy, ${ }^{35}$ or site selective chemical etching. ${ }^{36}$

The frequency and strength of the resonances are sensitive to the nanocube geometry (corner, edge sharpness), separation from the support, and inter-particle interactions. ${ }^{33}$ Therefore, despite the unusual and useful properties of supported silver nanocubes, their functional use remains challenging due to changes induced even by minor variations in their physical state. Nanocube rounding induced by sulfidation for example, alters the plasmon response significantly introducing a significant error in the measurements. ${ }^{37} \mathrm{~A}$ way to overcome the issue is to add a protective layer encapsulating the nanocubes into a solid matrix thus preventing chemical and physical alterations.

In the present work an unusually sharp and strong resonance in the extinction spectrum of supported silver nanocubes overcoated with a nanometer thick dielectric coating is observed. Properties of the resonance are probed and possible origin and applications are discussed. 


\subsection{Results and Discussion}

\subsubsection{Observation of sharp localized surface plasmon resonances}

Extinction spectra of supported silver nanocubes undergo dramatic transformations when overcoated with a thin dielectric layer (Figure 2.5). The two resonances observed for uncoated sample at 393 and $427 \mathrm{~nm}$ are hybrid $Q$ and $D$ respectively (Figure $2.5 \mathrm{a}$ ). With a stepwise increase of the overlayer thickness the resonances red shift, the $Q$ mode shifts further due it being located distal of the substrate. The resonances overlap when the alumina film thickness reaches $\sim 17 \mathrm{~nm}$ (120 ALD cycles, Figure 2.5d). At this point, a very strong and sharp resonance is observed at 459 $\mathrm{nm}$. With continued coating, the single peak modestly red shifts reaching saturation at $484 \mathrm{~nm}$ for $56 \mathrm{~nm}$ of alumina (400 cycles, Figure $2.5 \mathrm{~h}$ ).

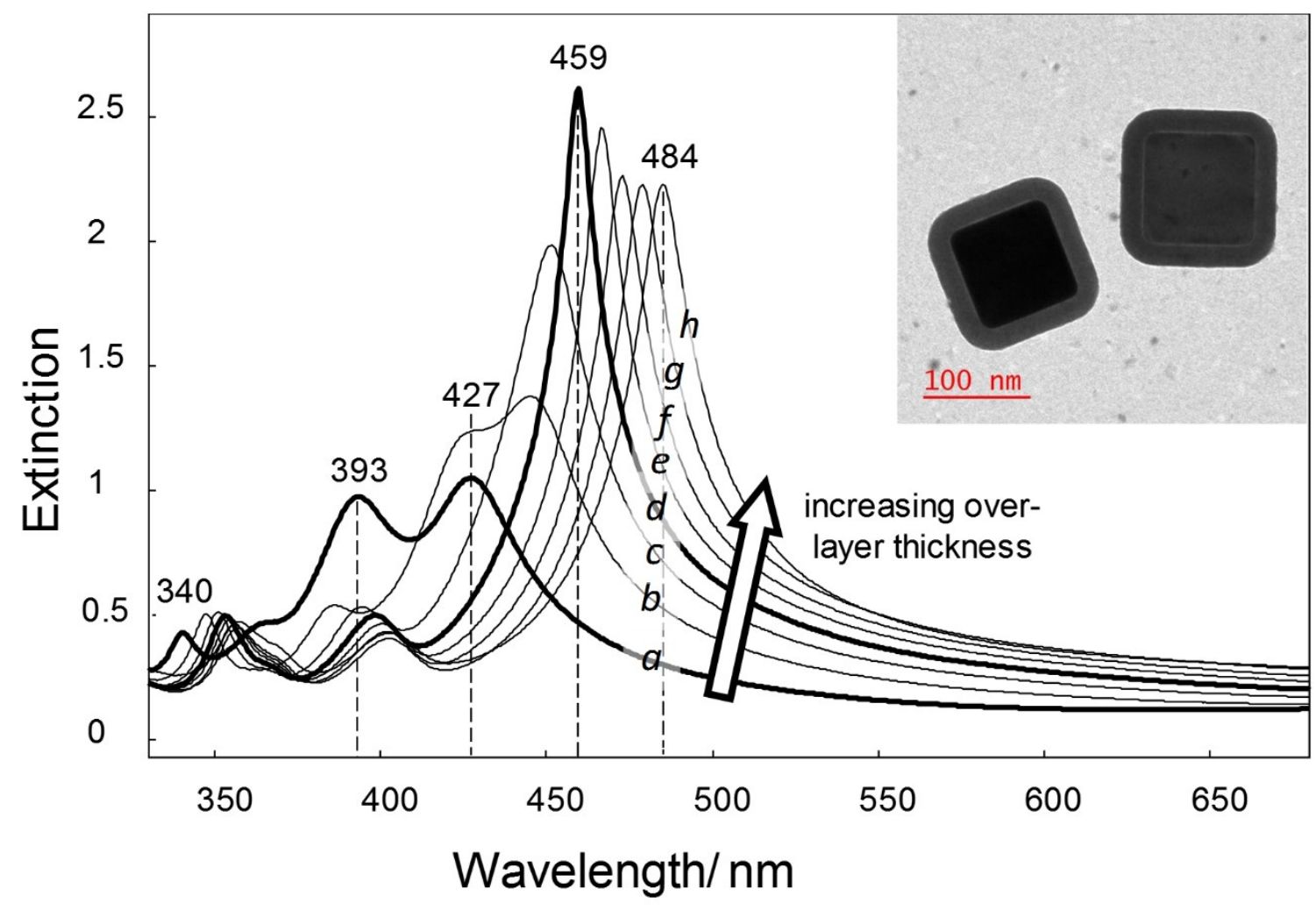

Figure 2.5. Extinction spectra of a silver nanocube monolayer as a function of overlayer coating. Silver nanocubes of $\sim 80 \mathrm{~nm}$ edge length (inset shows TEM image of silver nanocubes with $\mathrm{Al}_{2} \mathrm{O}_{3}$ shell), on a glass substrate are over-coated with a layer or $\mathrm{Al}_{2} \mathrm{O}_{3}$. Spectra were measured every 10 ALD cycles $(\sim 1.4 \mathrm{~nm})$ between 0 and 400 cycles (0 to $56 \mathrm{~nm}$ ). Spectra presented are for 0 (a, thick line), $40(5.6 \mathrm{~nm}, \mathrm{~b}), 80(11.2$ $\mathrm{nm}, \mathrm{c}), 120$ (16.8 nm, d, thick line), 160 (22.4 nm, e), $200(28 \mathrm{~nm}, \mathrm{f}), 260$ (36.4 nm, g), and 400 (56 nm, h) cycles. 
The red-shifting of the $D$ and $Q$ resonances is expected. The refractive index $(n)$ of alumina deposited via ALD is 1.7 at $500 \mathrm{~nm},{ }^{38}$ and greater than that of silica $(n=1.52)$. Therefore, with increasing thickness the overlayer effective refractive index eventually becomes equal to that of the underlayer, removing local refractive index anisotropy and conditions required for hybridization between the dipolar and quadrupolar modes. Consequent increase of the overlayer thickness leads to contrast inversion; however, the spectral effect is not as significant since LSPR sensitivity drops very rapidly with distance from the nanoparticle surface (Figure $2.5-\mathrm{h}$ ).

Of particular interest is a very narrow linewidth and increased strength of the resonance for the sample with $\sim 17 \mathrm{~nm}$ alumina overlayer (Figure $2.5 \mathrm{~d}$ ). Integrated extinction spectral intensity between $300-650 \mathrm{~nm}$ doubled when compared to uncoated sample. The maximum peak intensity exceeds 2.5 extinction units, or light transmittance of less than $0.3 \%$ (Figure B.4). The peak's full width at half maximum (FWHM) is around $20 \mathrm{~nm}$, or $130 \mathrm{meV}$ in this spectral range. This is considerably narrower than homogeneously broadened dipolar resonances of comparable individual nanoparticles ${ }^{39-41}$ whereas, for ensemble-averaged measurements, this represents a significantly sharp-LSPR (sLSPR). The unusual resonance narrowing promoted by the overlayer deposition contrasts with unchanged profiles of multipole resonances between 330 and $375 \mathrm{~nm} .^{23}$

\subsubsection{Optical anisotropy of SLSPR}

Another valuable property of supported silver nanocubes is their near and far field optical anisotropy. Due to local anisotropy of the effective refractive index around the nanocubes their plasmonic response is sensitive to the direction of nanocube illumination. ${ }^{35}$ While transmission of light is direction- independent (Figure B.1), reflection is very sensitive to illumination geometry (Figure B.2). A narrow dip observed for both $s$ and $p$ polarized light for front illumination geometry at $\sim 400 \mathrm{~nm}$ is not present for the rear illumination geometry. This translates into the difference in absorptance spectra (figure B.3): a very strong peak around $400 \mathrm{~nm}$ is present for front but not for rear illumination. The $20 \%$ differential absorptance (front minus rear) at a maximum clearly demonstrates the anisotropy Figure 2.6A. With deposition of a dielectric film, the anisotropy decreases and when the sLSPR is observed the differential absorptance signal drops to less than 4\% (Figure 2.6B). We can therefore conclude that the sLSPR appears under optically isotropic 
local conditions. This is confirmed by experiments with various support and overlayer materials used, including alumina, titania, and zirconia. In all cases, sharp and strong resonances could be observed under optically isotropic conditions.
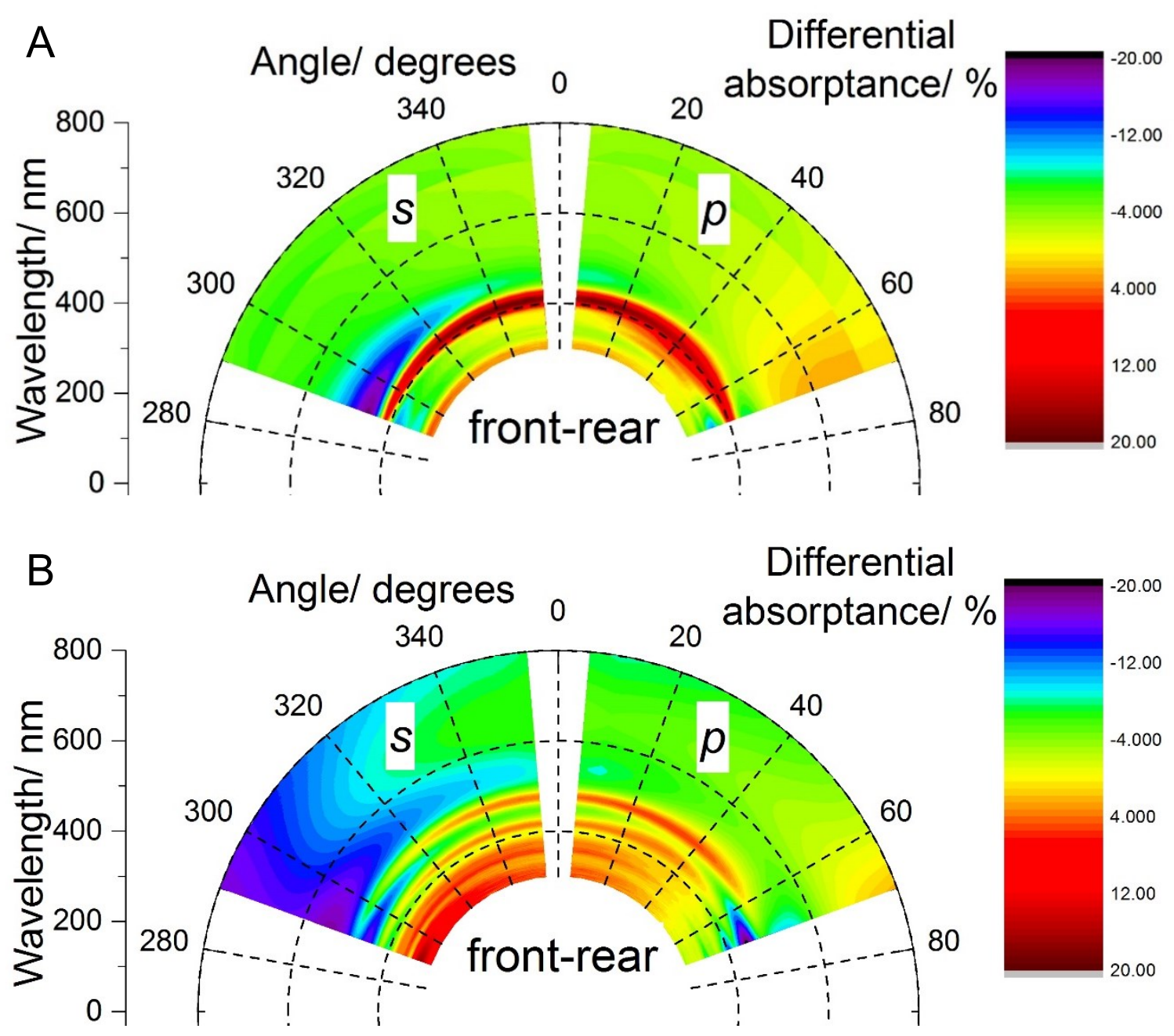

Figure 2.6. Differential absorptance (absorptance for front minus absorptance for rear illumination geometry) spectral maps for $(A)$ uncoated silver nanocubes on a glass substrate and $(B)$ silver nanocubes on a glass substrate overcoated with a thin film of alumina and exhibiting sharp LSPR. The spectral maps are obtained for $s$ (left) and $p$ (right) polarized light for angles of incidence between 5 and 70 degrees in 5 degree increments. Wavelength is plotted radially.

\subsubsection{SERS using SLSPR}

The plasmonic nature of the SLSPR was probed by measuring SERS excitation profiles. Two Raman labels were used: intrinsic polyvinylpyrrolidone (PVP) added as a protecting agent during the nanocube synthesis, and 4-mercaptobenzoic acid (MBA). The dielectric coating was deposited over the SERS labels until the SLSPR appeared. These SERS labels have no electronic transitions in the visible part of the spectrum and therefore their SERS excitation profiles should be 
defined by the wavelength dependence of the local electric field. MBA was deposited by immersing the glass supported silver nanocubes into MBA solution for 1 hour. This ligand exchange reaction results in full exchange of PVP with MBA, as confirmed by SERS. However, it also induces partial nanocube rounding, manifested in the extinction spectra as reduced peak hybridization (Figure B.7). This leads to generation of a less pronounced SLSPR, with significantly weaker and broader resonance, but the same resonance frequency (Figure B.7). The untreated with MBA counterpart sample showed very strong and narrow resonance (Figure B.7). The two samples were further studied to compare the effect of the sLSPR on SERS excitation profiles. SERS peaks at $\sim 1590$ and $\sim 1760 \mathrm{~cm}^{-1}$ (Figure B.8) were used to construct the profiles for MBA and PVP respectively.

The SERS excitation profiles (Figure 2.7, red dots) show a general trend of increasing towards longer wavelength with respect to the extinction spectra. This is likely due to a small number of nanocube clusters (dimers, trimers) that are inevitably present in the system and have red shifted coupled plasmon mode ${ }^{9}$ that provides most of the SERS signal, yet are mute in UV-vis extinction spectrum. For the PVP sample, there is a local maximum in the SERS excitation profile around the SLSPR peak signalling enhanced local E-field around the cubes as opposed to the MBA sample. The data indicate that for samples with pronounced sLSPR not only far field interaction of the nanocubes with light are enhanced, as demonstrated by the light extinction data, but also the near field.
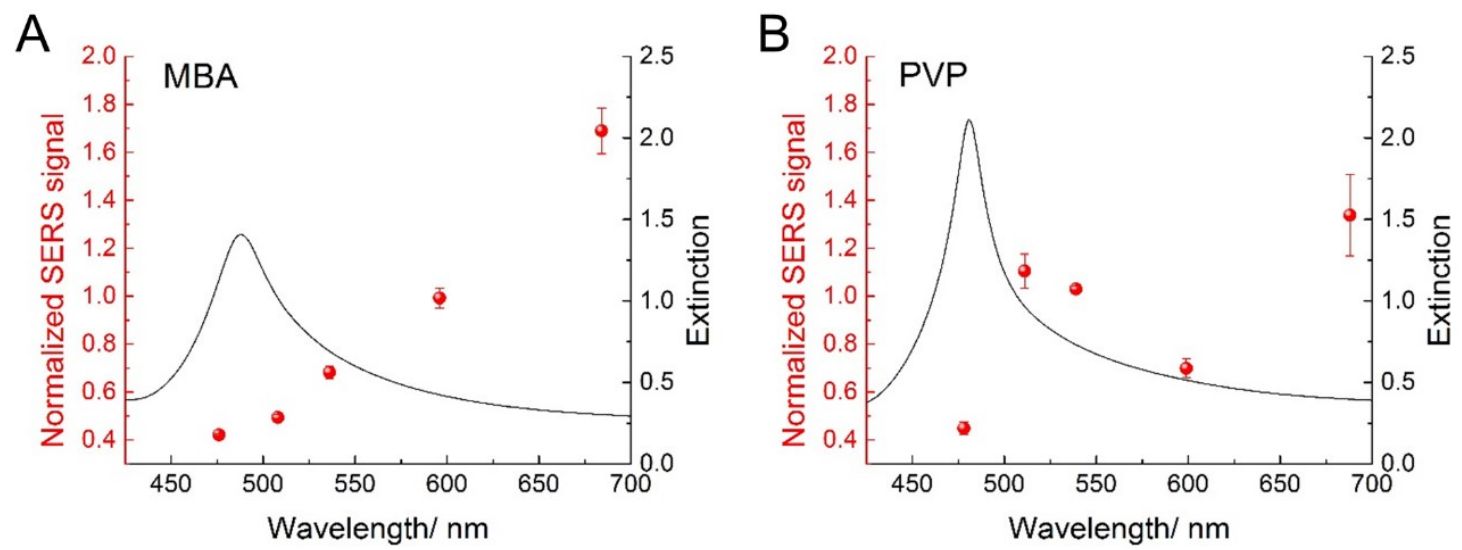

Figure 2.7. SERS excitation profiles for 4-mercaptobenzoic acid (MBA, left) and polyvinylpyrrolidone (PVP, right) overlaying extinction spectra (black curves), taken at 5 different excitation wavelengths, $458 \mathrm{~nm}, 514$ $\mathrm{nm}, 568 \mathrm{~nm}$, and $647 \mathrm{~nm}$. SERS intensity is normalized to an external reference and plotted at the Stokesshifted wavelength. 


\subsubsection{Origin of LSPR narrowing}

Very narrow LSPRs for ensembles of nanoparticles have previously been observed in several experimental studies and could be attributed to collective plasmon coupling, ${ }^{42,43}$ coherent interparticle plasmon coupling ${ }^{44}$ or coherent mode coupling. ${ }^{18}$

Collective plasmon coupling is observed for ordered arrays of plasmonic nanoparticles. If for such a system, a diffraction mode propagating in plane of the nanoparticle array has a wavelength coinciding with the nanoparticle LSPR collective coupled resonances will be observed. The spectral width of such resonances can be as narrow as several nanometers. ${ }^{42,43}$ However, long range order in the system is required, which is not the case here.

Coherent interparticle plasmon coupling results in a formation of a very sharp and strong resonance. ${ }^{44}$ It was observed for random nanoparticle arrays where the particles are spaced close enough to provide near-field interactions. This is not the case of our system, as the nanoparticle spacing is large since the interparticle coupling mode is not present in the extinction spectrum.

We therefore conclude that the most likely origin of the very narrow resonance observed in this work is coherent mode coupling. More specifically, interference between the dipolar and quadrupolar modes in each nanocube, similar to Fano interference. ${ }^{18}$ Without the overlaying dielectric film, the resonance frequencies of the $Q$ and the $D$ modes differ, and the modes are spatially separated on opposite ends of the nanocube (Figure B.9, A). As the effect was observed for nanocube sizes between 60 and $80 \mathrm{~nm}$, about $\lambda / 6$, phase delay between excitation of the two modes becomes non-negligible, as was shown previously for some other nano-architectures. ${ }^{45-48}$ The Fano-like origin of the interference between the two modes is supported by a characteristic dip in the extinction spectrum..$^{18}$ The dipolar and quadrupolar nature of the two resonances is also quite distinct (Figure 2.8A). 
A
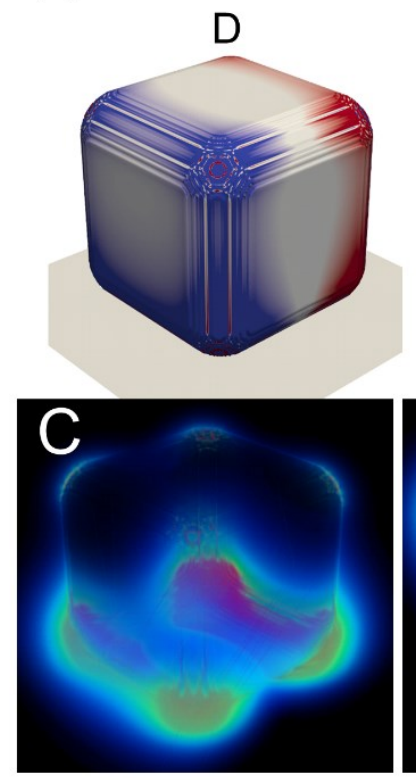

$0 \mathrm{~nm} \mathrm{Al} \mathrm{O}_{3}$
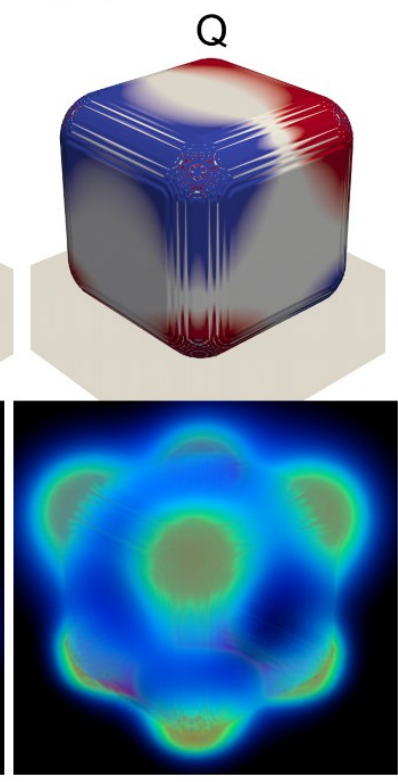

B
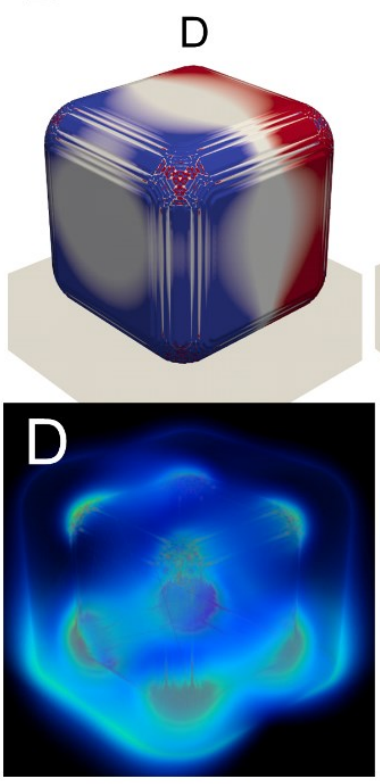

$12.5 \mathrm{~nm} \mathrm{Al}_{2} \mathrm{O}_{3}$
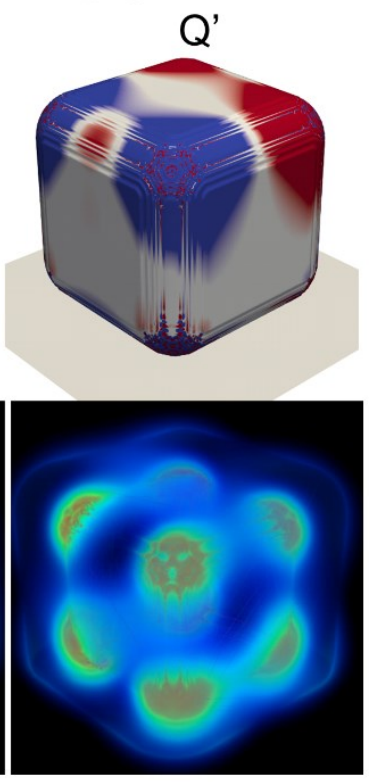

Figure 2.8. Calculated density, $\rho$, and electric field intensity, $|\mathrm{E}|^{2}$ for $\left.A, C\right) 0 \mathrm{~nm} \mathrm{Al} 2 \mathrm{O}_{3}$ and $\left.\mathrm{B}, \mathrm{D}\right) 12.5 \mathrm{~nm} \mathrm{Al}_{2} \mathrm{O}_{3}$ respectively. Originally the $D$ mode lies within the substrate and the $Q$ mode outside, but as $A_{2} \mathrm{O}_{3}$ is overcoated the $D$ and $Q$ modes become isotropic, and the $Q$ ' begins to take on a stronger $D$ character.

With increasing overlayer thickness both spectral and spatial separation between the two modes decrease (Figure B.9, b-f]. As the substrate and superstrate resemble a more homogeneous dielectric environment $Q$ and the $D$ modes overlap spectrally (Figures B.9, c-e). In the model the FDTD full coincidence of the peaks are not observed due to limitations in predicting surface effects on the magnitude of the refractive index which effectively varies with thickness. ${ }^{38}$ Nevertheless the model demonstrates that the modes spatially become much closer (Figure 2.8). As a result, the phase delay effect is removed, and the interference becomes constructive. At the same time, the $Q$ mode character changes from mostly quadrupolar to essentially dipolar (Figure 2.8). Such constructive interference is the most likely reason for the narrowness of the resonance in the extinction spectrum, and its overall strength, similar to electromagnetically induced absorption. ${ }^{45}$ It is quite possible that reduced reflection, ${ }^{49}$ also plays a role. This follows from the data presented in B.1 - B.6 for reference, where a narrow resonance is present in the reflectance rather than the absorbance spectra. It is important to note that rounding of the nanocubes affects both spatial and spectral characteristics of $Q$ and $D$, leading to decrease of constructive interference (Figure 2.7). As is concluded form the SERS measurements, the new narrow peak 
provides stronger E-field around the nanoparticle. Since the system becomes more isotropic, direction of light does not play a role in the observation of the SLSPR.

\subsection{Conclusion}

An unusually sharp localized surface plasmon resonance for an ensemble of supported silver nanocubes is observed. The resonance most likely results from a coherent constructive interference between the dipolar and quadrupolar modes. The phenomenon appears to be universal and was observed for various supporting and overlaying materials. As the resonance is buried deep inside the dielectric, applications such as photoinduced catalysis in solids, optical filters or photovoltaics are most likely to benefit from the phenomenon. 


\section{Hybridized plasmon resonances in core/half-shell cuprous oxide/silver nanoparticles: towards multi- functional plasmonic nanoparticles}

\subsection{Part C: Introduction}

Core/shell nanoparticles with chemical and/or morphological anisotropy find many uses due to their attractive optical, physical and catalytic properties stemming from the interaction of the core and shell. Applications that have benefited from these properties include self-motile microparticles,${ }^{50}$ biological sensors, ${ }^{51}$ optical sensors, ${ }^{52}$ and building blocks for complex structures. ${ }^{53}$ Metal/semiconductor core/shell particles show particular promise for photocatalytic applications, ${ }^{54}$ where the plasmonic particle can act as a photosensitizer and provide charge carriers for chemical reactions at the semiconductor surface.$^{55}$ One such system is the use of a silver core with a cuprous oxide shell $\left(\mathrm{Ag} @ \mathrm{Cu}_{2} \mathrm{O}\right) .{ }^{56,57}$ Additional opportunities appear when the shell of the nanomaterial does not fully cover the core. In this case, Janus-like particles or core/half-shell nanoparticles can allow for directed assembly, ${ }^{58}$ or regiospecific surface chemistry. ${ }^{59}$

An anisotropic shell around a nanoparticle can also be used to control the plasmonic response. An asymmetric dielectric environment around metal particles, such as a silver nanocube, produce spectrally and spatially separated hybridized plasmon modes. ${ }^{18}$ This phenomenon has only been reported for supported nanoparticles, however hybridization and spatio-spectral control of the plasmon modes for nanoparticles should be achievable in any partial dielectric environment, and the utilization of such modes has many potential applications. For example, manipulation of the spatial arrangement of plasmon modes can be of benefit to site-specific photocatalysis. ${ }^{36}$

There are a few existing instances of this inorganic core/half-shell morphology, of which gold cores with polymer, palladium, or silica shells have been synthesized. ${ }^{58,60-63}$ These were made using either ligand competition, ${ }^{59,60}$ water-in-oil microemulsions forming a sacrificial silica mask, ${ }^{61}$ spin casting of a nanoparticle/polymer mixture forming a partial mask, ${ }^{62}$ or a solid interface to hinder growth, ${ }^{63}$ these methods are typically restricted in the material of core and shell that can be used and have a limited ability to alter the amount of core coverage. ${ }^{58-63}$ 
To address these challenges, this work proposes a reliable method for the formation of core/half-shell particles. Silver nanocube $(\mathrm{AgNC}) / \mathrm{Cu}_{2} \mathrm{O}\left(\mathrm{AgNC} @ \mathrm{Cu}_{2} \mathrm{O}\right)$ core-shell nanoparticles are synthesized according to Figure 2.9. The AgNCs are first deposited onto the surface of a polystyrene (PS) film using Langmuir-Schaeffer method and are then gradually embedded into the PS film via the heating process described in chapter 0 until the desired depth is reached. This is followed by the liquid phase growth of the $\mathrm{Cu}_{2} \mathrm{O}$ half shells by the formation of $\mathrm{Cu}(\mathrm{OH})_{2}$ from a $\mathrm{Cu}(\mathrm{II})$ salt, followed by reduction to $\mathrm{Cu}(\mathrm{I})_{2} \mathrm{O}$ by hydrazine. ${ }^{64}$ The $\mathrm{Cu}_{2} \mathrm{O}$ only grows on the exposed part of the nanocube, and the core/half-shell AgNC@ $@ \mathrm{Cu}_{2} \mathrm{O}$ particles are re-dispersed in solution by dissolution of the PS film in toluene.
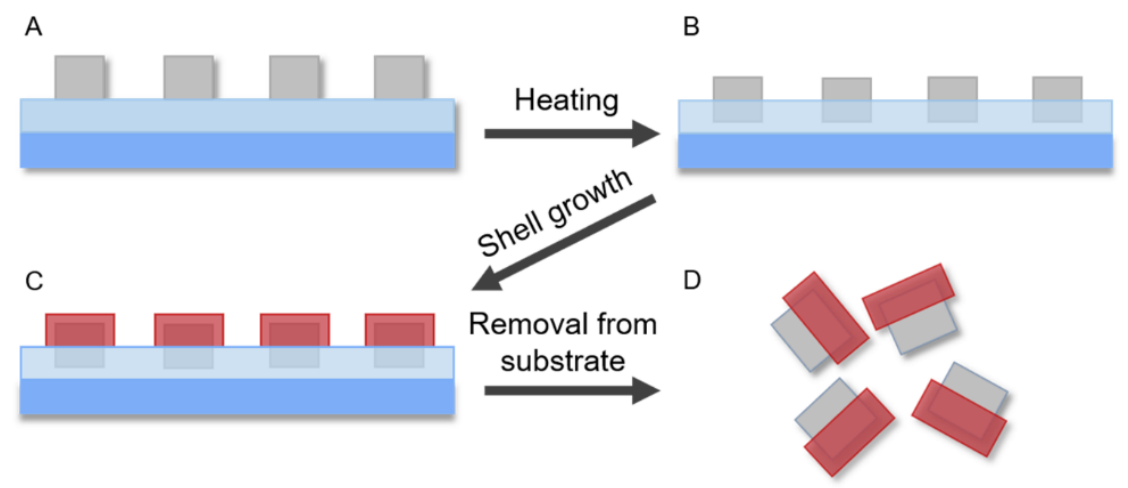

Figure 2.9. Schematic of the core/half-shell synthesis methodology. (A) Silver nanocube (AgNC) monolayer on a polystyrene (PS) thin film supported by glass. (B) AgNCs partially embedded into PS through heating. (C) $\mathrm{Cu}_{2} \mathrm{O}$ half-shells grown on the exposed portion of the AgNCs. (D) $\mathrm{AgNC} / \mathrm{Cu}_{2} \mathrm{O}$ core/half-shell nanoparticles removed from substrate by dissolution of PS with toluene.

The growth of $\mathrm{Cu}_{2} \mathrm{O}$ on $\mathrm{Ag}$ is epitaxial, growing from the $\{111\}$ or $\{100\}$ facets of the AgNC. ${ }^{65,66}$ Truncated silver nanocubes have a face-centered cubic crystal lattice with the $\{100\}$, $\{110\}$, and $\{111\}$ facets exposed at the faces, edges, and corners of the nanocube respectively. ${ }^{67}$ Cuprous oxide has a cuprite structure: body-centered cubic packing of oxygen atoms with copper atoms occupying one-half of the tetrahedral sites. ${ }^{68,69}$ Silver and cuprous oxide have the lattice parameters of $4.090 \AA$ and $4.269 \AA$, respectively, resulting in a lattice mismatch of $4.4 \% .^{70}$ This is sufficient to enable the $\mathrm{Cu}_{2} \mathrm{O}$ to form via heterogeneous nucleation followed by epitaxial growth from the Ag surface. ${ }^{71}$ This process is in contrast to the hollow-shell-refilled (HSR) mechanism wherein $\mathrm{Cu}_{2} \mathrm{O}$ seeds are first formed in solution and then aggregate to form cage-like partial shells that are connected to the core via $\mathrm{Cu}_{2} \mathrm{O}$ bridges. The core is then able to guide growth and the shell is filled. ${ }^{66}$ This typically produces octahedral shells, preferentially growing off $\mathrm{Ag}\{111\} .{ }^{65,66,71}$ 
There are many parameters that will affect the morphology of $\mathrm{Cu}_{2} \mathrm{O}$ nanoparticles such as reagent concentrations, their order of addition, and environmental conditions such as $\mathrm{pH}$. $\mathrm{Cu}_{2} \mathrm{O}$ nanocrystals have been synthesized with cubic, truncated cubic, cuboctahedral, truncated octahedral, octahedral, and short hexapod morphologies. ${ }^{72}$ This work explores a number of these parameters and their effect on controlling the morphology of the produced $\mathrm{Ag} @ \mathrm{Cu}_{2} \mathrm{O}$ half-shell composite particles. The experimental methods to produce these particles are beyond the scope of this thesis, but some of the produced morphologies and their optical properties will be showcased. The growth-processes of these particles was investigated using optical signature produced evolution of the hybrid plasmonic modes. Numerical modelling was used to explore the origins of the modes, such as in determining their order and spatial distribution, as well as to predict the spectral characteristics of ideal core/half-shell $\mathrm{Ag} @ \mathrm{Cu}_{2} \mathrm{O}$ particles.

\subsection{Results and Discussion}

\subsubsection{Depth of embedment/ $\mathrm{Cu}_{2} \mathrm{O}$ coverage}

Utilisation of the thermal embedment strategy for partially coating the nanocubes allows one to choose the fraction of AgNC exposed for growth of shell. This can potentially tune the placement of the shell component relative to the core, allowing one to tune the exposed surface area of the core component. Figure 2.10A,B displays transmission electron micrographs and extinction spectra of AgNC@ $@ \mathrm{Cu}_{2} \mathrm{O}$ shells grown with different amounts of the cube exposed. The progression of embedment impacts the area of shell coverage, resulting in coverage of the faces or the corners. Corner-centered growth is particularly observed for particles that were embedded greater than half-way the height of the cube. In the thermal embedment process the nanocubes tilt as the embed $>50 \%$ into the substrate. ${ }^{27}$ At shallow embedment depths, the shell grows as a truncated pyramid, with the bottom of and top faces of the pyramid aligned parallel to the top face of the nanocube. The pyramid faces are observed to be rotated $45^{\circ}$ relative to the cube faces.

From the extinction spectra of the $\mathrm{AgNC} / \mathrm{Cu}_{2} \mathrm{O}$ nanoparticles in toluene, the plasmonic signature, in particular the peak splitting between the modes labelled as Q ( 460 nm) and D ( 680 $\mathrm{nm}$ ) was not found to deviate significantly with respect to variation in embedment depth (and 
therefore surface coverage of the $\mathrm{AgNC}$ with $\mathrm{Cu}_{2} \mathrm{O}$ ). The $\mathrm{Q}$ mode changes in intensity relative to the D-mode however, increasing in intensity as the nanoparticle is further embedded (Figure 2.10C), and is thus less exposed to the $\mathrm{Cu}_{2} \mathrm{O}$.

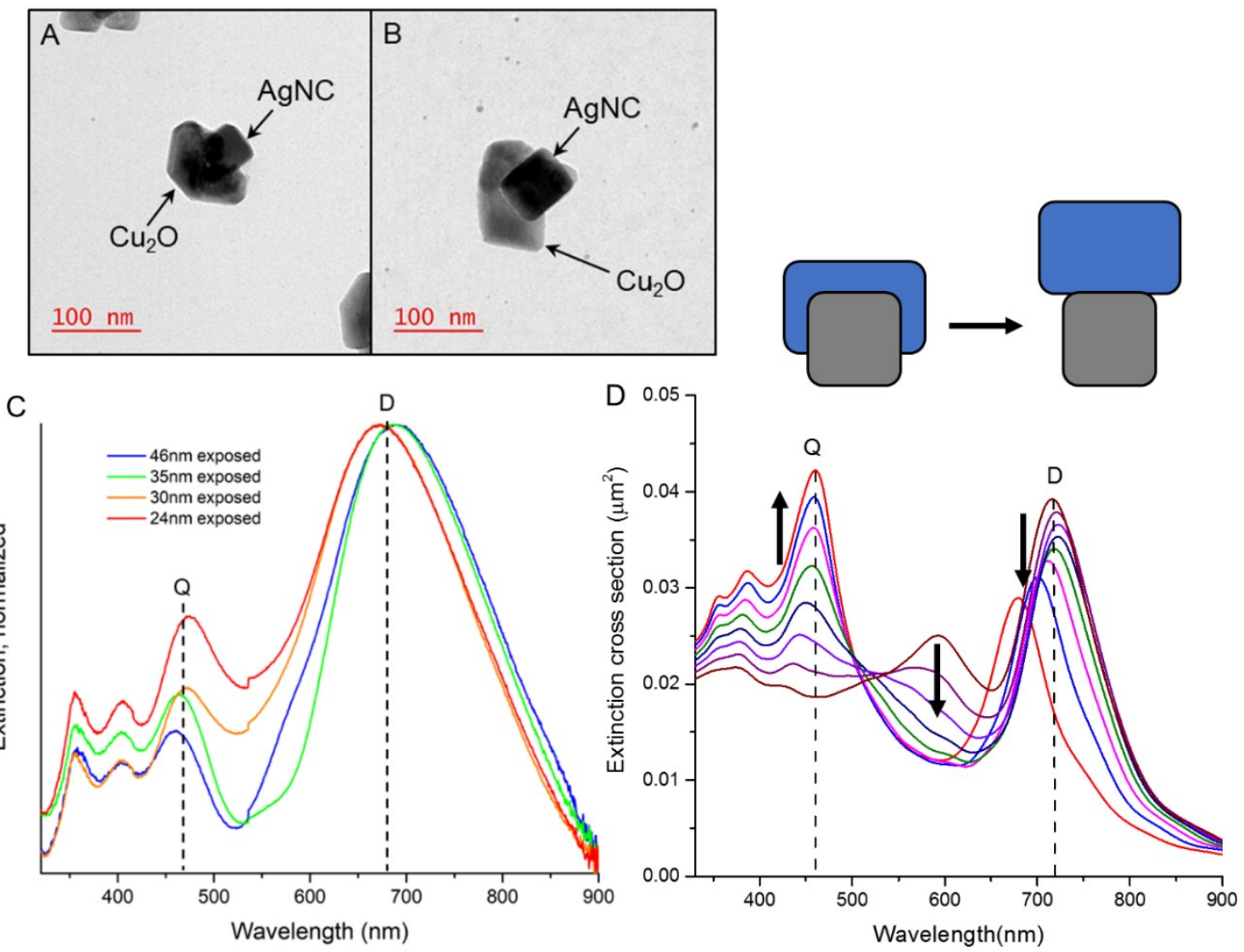

Figure 2.10. TEM images of $\mathrm{Ag} @ \mathrm{Cu}_{2} \mathrm{O}$ grown when the $\mathrm{AgNC}$ was: $\mathrm{A}$ ) less than half-embedded and $\mathrm{B}$ ) more than half-embedded. B) Evolution of the colloidal extinction spectra at different embedment heights C) FDTD model of an in-solution nanocube with varying heights of a cubic $\mathrm{Cu}_{2} \mathrm{O}$ shell.

FDTD modelling was used to examine the relationship of the height of the shell on the particle. The model consisted of a $20 \mathrm{~nm}$ thick rectangular with varying relative heights on the AgNC in $5 \mathrm{~nm}$ steps. A rectangular shell, rather than a pyramidic shell, was since a geometry mismatch in the system skewed results at high "embedment depths"; the corners of the nanocubes would not be covered by the $\mathrm{Cu}_{2} \mathrm{O}$ shell at some shell heights (Figure C.1). The modelled extinction spectra initially show a similar position and insensitivity of the $Q$ and $D$ positions at $\sim 450 \mathrm{~nm}$ and $\sim 700 \mathrm{~nm}$, respectively, as in the experimental spectra. The model also displays a decrease in intensity of the $\mathrm{Q}$ mode as the coverage of $\mathrm{Cu}_{2} \mathrm{O}$ increases in agreement with the experimental result. The 
simulated extinction profile looks very different at high coverages of $\mathrm{Cu}_{2} \mathrm{O}$ however. The $\mathrm{Q}$ mode all but vanishes, and, curiously, a new mode around $600 \mathrm{~nm}$ appears also appears in the model.

\subsubsection{Spatial distribution of plasmon modes}

To better understand the origin of the plasmonic modes in the $\mathrm{Cu}_{2} \mathrm{O}$, and explain the discrepancies in the model, the spatial distribution of the plasmon modes was investigated. Figure 2.10 shows the charge density, $\rho$, and electric field enhancement, $|\mathrm{E}|^{2}$ of the observed peaks for a $20 \mathrm{~nm}$ shell embedded covering $66 \%$ the height of the cube.
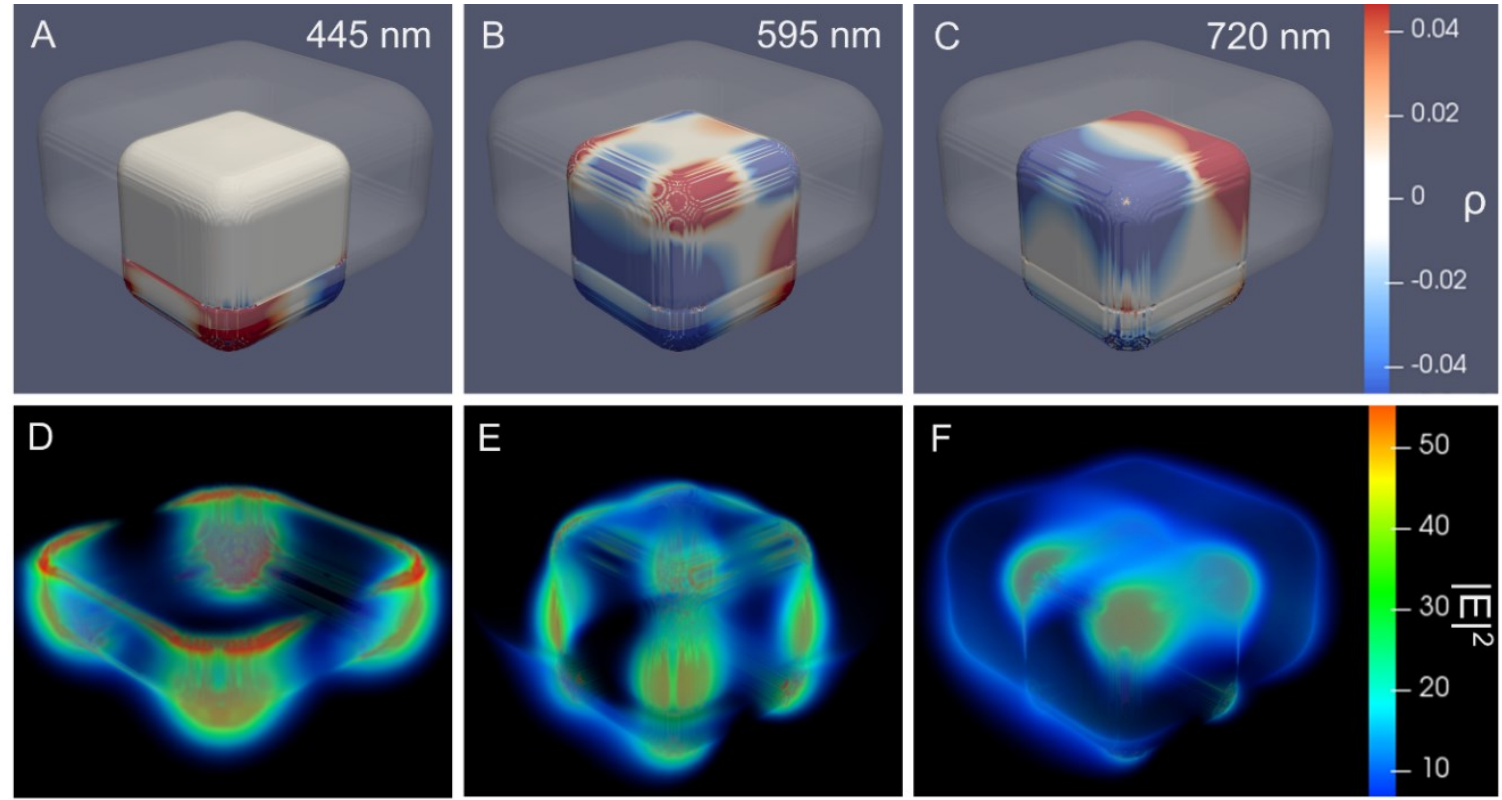

Figure 2.11. 3D view of the charge density, $\rho$, and electric field enhancement, $\left|\mathrm{E} / \mathrm{E}_{0}\right|^{2}$, of plasmonic modes at a wavelength of $A, D)$ the "Q" mode at $445 \mathrm{~nm}, \mathrm{~B}, \mathrm{E})$ the multipolar $\left(\mathrm{M}^{*}\right)$ mode at $595 \mathrm{~nm}$, and $\left.C, F\right)$ the "D" mode $720 \mathrm{~nm}$ of a nanocube in which the $\mathrm{Cu}_{2} \mathrm{O}$ shell covers $2 / 3$ of the surface.

The dipolar resonance (D) Figure $2.11 \mathrm{C}$ is located within the higher index medium of the $\mathrm{Cu}_{2} \mathrm{O}$ as expected. ${ }^{23}$ However the other two modes are anomalous in regards to typical descriptions of silver nanocubes. The mode at $445 \mathrm{~nm}$, which is expected to be the quadrupolar $(\mathrm{Q})$ resonance and is located in the lower index medium as the $Q$ mode is expected to be yet seems to actually be dipolar in nature. Peculiarly, the mode is located almost exclusively outside of the $\mathrm{Cu}_{2} \mathrm{O}$ shell, with a near zero $\rho$ and negligible $\left|\mathrm{E} / \mathrm{E}_{0}\right|$ located within the $\mathrm{Cu}_{2} \mathrm{O}$. This phenomenon is likely due to the high imaginary component in the dielectric function of $\mathrm{Cu}_{2} \mathrm{O}$ in this region of the spectrum; this 
mode is located energetically above the band gap of $\mathrm{Cu}_{2} \mathrm{O}$ which is spectrally located $\sim 560$ $\mathrm{nm}{ }^{70,73,74}$ This would lead to dielectric losses in the $\mathrm{Cu}_{2} \mathrm{O}$ phase of the system.

The anomalous new mode at $~ 590$ appears to be a hybrid hexapolar mode with a near uniform spatial distribution inside and outside the $\mathrm{Cu}_{2} \mathrm{O}$ shell, concentrated at the edges of the nanocube. This is unusual as higher-order modes tend to occur at higher energies relative to loworder modes, ${ }^{1}$ yet this mode occurs at a lower energy than the $Q$ mode. While this new mode is not observed in Figure 2.10, in follow up experiments it was observed at high coverages of $\mathrm{Cu}_{2} \mathrm{O}$. Figure 2.12 shows the spectra of colloidal $\mathrm{Ag} @ \mathrm{Cu}_{2} \mathrm{O}$ half shells made at $\sim 50 \%$ embedment with varying concentration of the precursors $\mathrm{Cu}(\mathrm{NO})_{3}, \mathrm{NaOH}$, and lactic acid. From a $-\mathrm{g}$ these precursors increase in total reaction concentration, maintaining the same relative amounts to each other. At high coverages a new mode $\left({ }^{*}\right)$ can be faintly observed $\sim 580 \mathrm{~nm}$, confirming the theoretical prediction of its existence.
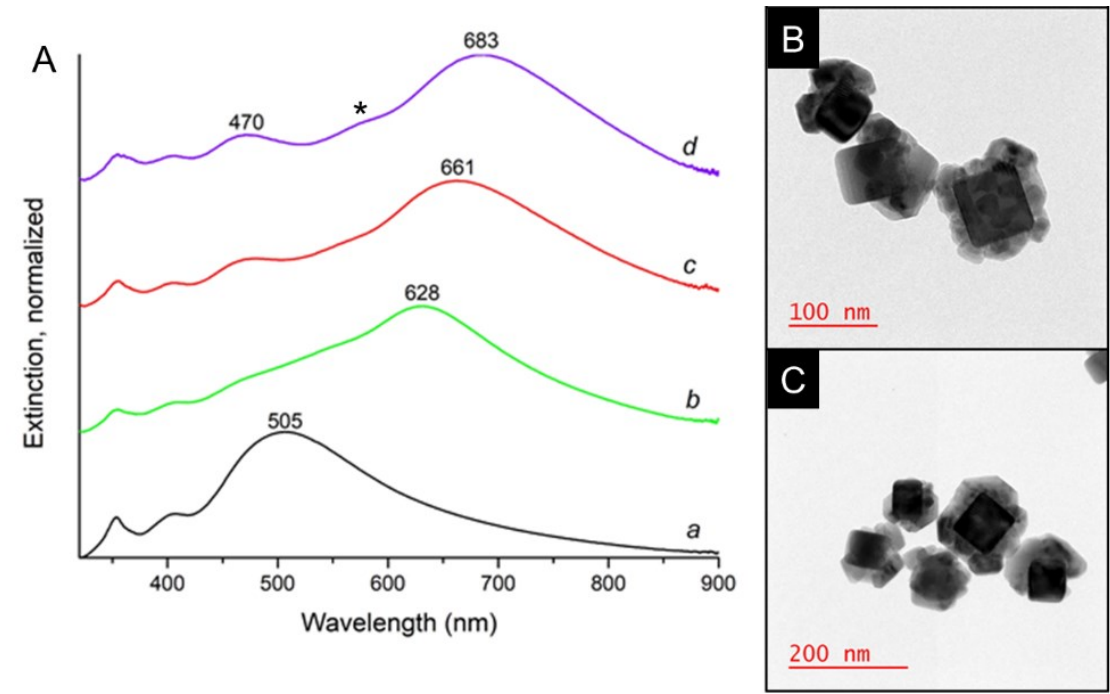

Figure 2.12. $\mathrm{Cu}_{2} \mathrm{O}$ half-shell growth of controlled sizes with increasing strength of reagents from starting from 0 (a) $-42.5 \mathrm{mM} \mathrm{Cu}\left(\mathrm{NO}_{3}\right)$ showing the A) evolution of the UV-vis extinction profile with increasing reagent concentration, highlighting observation of the new $\left(^{*}\right)$ multipolar mode. TEM images are provided for lines B) (b) and C) (d).

The UV-Vis extinction spectra contain qualitatively the same peaks as the modelled extinction displayed in Figure 2.10D, though the overall spectral profile is not resembled. This is likely explained by the polydispersity in the sample's size, geometry and position of the $\mathrm{Cu}_{2} \mathrm{O}$ shells. Observing the TEM images in Figure 2.12 B,C the coverage of the $\mathrm{Cu}_{2} \mathrm{O}$ shells is far from uniform, 
and does not often extend past $50 \%$ of the edge length of the cube. The higher order mode is only observed at high ( $>40 \%$ ) coverages of $\mathrm{Cu}_{2} \mathrm{O}$ in the mode, assuming a uniform shell.

\subsubsection{Optimization of geometric parameters and description of peak shifts}

To better model the experimentally produced system, the geometric parameters of the model were adjusted to better resemble the produced nanostructures (Figure 2.13). In this model a truncated half-pyramid was used with rounded corners. While quantitatively (peak positions, intensities) are somewhat different than the experimental results (Figure 2.12), qualitatively the number of peaks, their behaviour with increasing shell thickness, more closely resemble the experiment than the previous model. It should be noted under some conditions, $\mathrm{Ag} @ \mathrm{Cu}_{2} \mathrm{O}$ halfshells with a conformal cubic geometry were produced (Figure C.2).
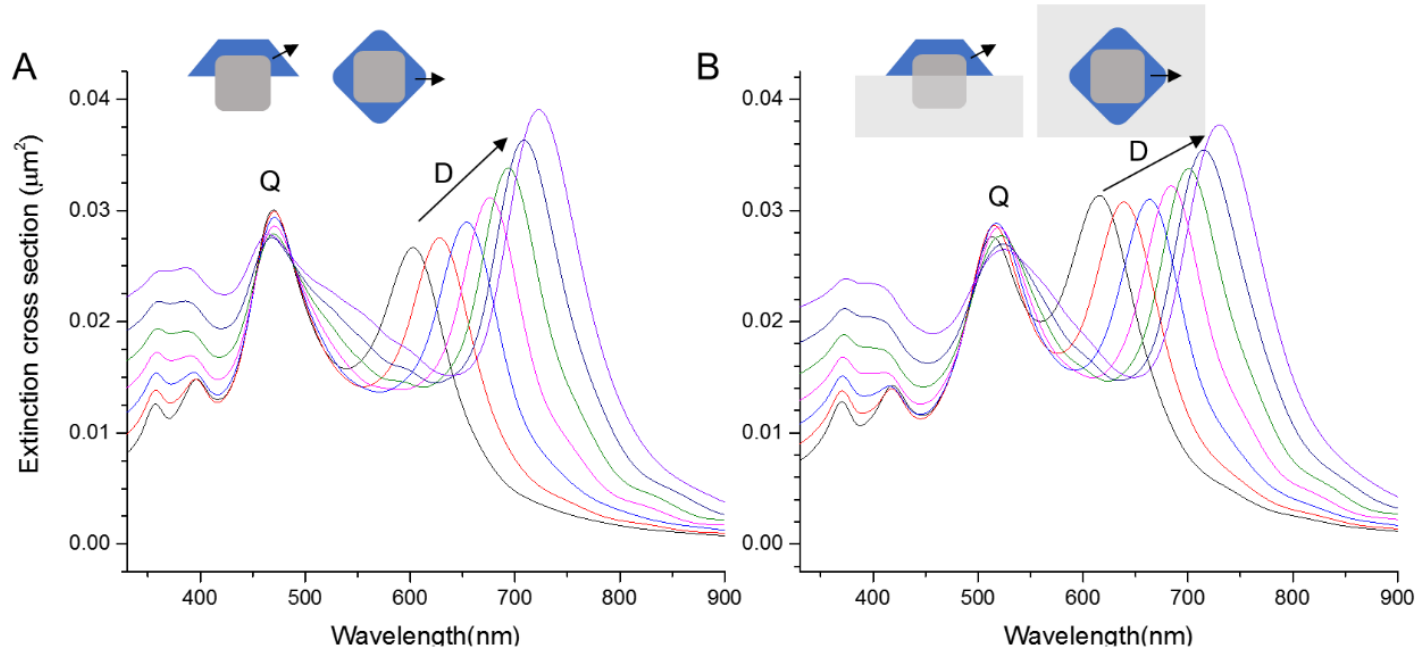

C
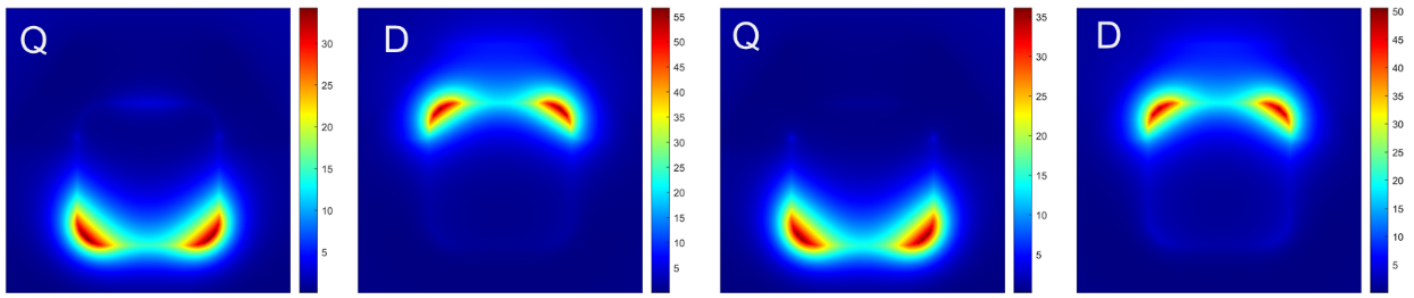

Figure 2.13. Modelled extinction of growing $\mathrm{Cu}_{2} \mathrm{O}$ truncated pyramid half-shells $\mathrm{A}$ ) in solution and $\mathrm{B}$ ) on polystyrene as they are first synthesized. C) associated $|E|^{2}$ with intermediate sized shells (cube face to pyramid edge $=30 \mathrm{~nm}$ ), displaying the dipolar $(\mathrm{D})$ and quadrupolar $(\mathrm{Q})$ modes.

The silver nanocubes initially embedded in polystyrene display strong mode splitting, with the dipolar (D) mode at $\sim 500 \mathrm{~nm}$ located strongly within the substrate, and the quadrupolar (Q) mode at $\sim 400 \mathrm{~nm}$ above it. As the nanocube is over-coated in $\mathrm{Cu}_{2} \mathrm{O}$, the environment first becomes more isotropic for thin $\mathrm{Cu} 2 \mathrm{O}$ thicknesses before the contrast is inverted for thicker $\mathrm{Cu}_{2} \mathrm{O}$ films. Once 
the system becomes inverted system, the $\mathrm{D}$-mode is centered in the $\mathrm{Cu}_{2} \mathrm{O}$ and is strongly redshifting with increasing shell thickness (Figure 2.13B). The $Q$ mode at $\sim 500 \mathrm{~nm}$ is spatially located primarily in the PS film and is shell thickness independent. In solution the environment is less isotropic, and the dipolar resonance shifts with a greater sensitivity with respect to increasing $\mathrm{Cu}_{2} \mathrm{O}$ thickness (Figure 2.13C). The $Q$ peak however is almost shell thickness independent and is blueshifted to $\sim 475 \mathrm{~nm}$ as compared to in PS embedded state. The blue shift is explained by the spatial location of the $Q$ peak at the facet of the cube exposed to the solvent (toluene), which has lower refractive index than PS. Overall results of the modelling demonstrate good agreement with the experiment (Figure 2.12A,d). 3D electric field distribution and charge separation for each mode demonstrate that these modes are indeed quadrupolar and dipolar in nature (Figure C.3).

\subsection{Conclusions}

The plasmonic response of $\mathrm{Ag} @ \mathrm{Cu}_{2} \mathrm{O}$ half-shells is described by FDTD modelling. The models confirm the dipolar and quadrupolar nature of the modes observed experimentally. The modelling also suggested the presence of an unusual, low energy, high order hybrid plasmonic mode which to my knowledge has not been reported before in the literature, and experimental evidence supported the existence of the mode. While the experimentally produced half-shells showed large polydispersity in geometry and size, the models were adequate in determining the origin of the observed hybrid plasmonic modes. The work presents methodology that allows for the spatial separation of plasmonic modes in a core shell geometry which may be tailored to satisfy a multitude of uses including LSPR sensing, SERS, and photocatalysis. 


\subsection{Methods}

\subsubsection{Numerical Modelling}

Numerical Modelling. Finite difference time domain modelling was performed using Lumerical Solutions Inc. (FDTD Solutions v8.15-v8.17. An $800 \mathrm{~nm}$ cubic simulation space was used with a perfect matched layer (PML) boundary (12 layers). A total-field scattered-field (TFSF) light source was used, injecting light from above, perpendicular to the substrate (if present), absorption and scattering cross sections were measured from power transmission monitors in the scattered field and total field areas respectively. Extinction was calculated as the sum of absorption and scattering cross sections. Frequency-domain field profile monitors were used to calculate $|E|$. A non-uniform mesh was used, with a mesh override region containing a $0.5 \mathrm{~nm}$ mesh size extended at least 10 $\mathrm{nm}$ past the nanostructures. All dielectric-dielectric and metal-dielectric boundaries were treated with Lumerical's Conformal Mesh Technology (CMT), a proprietary extension of the Yu-Mittra method. ${ }^{75}$ The materials were described by an analytical model fitted to tabulated permittivity data of silver, polystyrene, $\mathrm{PVP}, \mathrm{Cu}_{2} \mathrm{O}$, and $\mathrm{Al}_{2} \mathrm{O}_{3} \cdot{ }^{28,73,76,77}$

The simulation objects consisted of a $60 \mathrm{~nm}$ silver nanocube with a corner radius of $12 \mathrm{~nm}$ and a $2 \mathrm{~nm}$ capping layer of PVP (unless an oxide shell was present in the model).

Models of nanocube embedment. Supported by a $150 \mathrm{~nm}$ film of PS supported by an arbitrarily large $\mathrm{SiO}_{2}$ substrate. The nanocube partially embedded into the substrate and rotated in one or two axes.

Models of unusually sharp resonances. Models of cubes overcoated with a dielectric consisted of a larger round cube with $0-25 \mathrm{~nm}$ thickness of $\mathrm{Al}_{2} \mathrm{O}_{3}$. Shells of an arbitrary refractive index $(n)$ between $1.5-2.0$, were tested, as well as alternative dielectrics, $\mathrm{TiO}_{2}$ and $\mathrm{ZrO}_{2}$.

Modelling of $\mathrm{Cu}_{2} \mathrm{O}$ coated cubes For $\mathrm{Cu}_{2} \mathrm{O}$ coated cubes, the morphology of the half shell was either a rounded cube with a thickness between $0-20 \mathrm{~nm}$ or truncated, round-edged square pyramid with a center-corner radius of $50-90 \mathrm{~nm}$. The anisotropically-coated cube was either in a background index of $n=1.36$ to represent a solution, or partially-embedded into polystyrene with a background index of $n=1$. 
Convergence testing. All models were tested for convergence by varying the distance to the PML, where $800 \mathrm{~nm}$ was found to be sufficiently far way to not observe reflections. Convergence was also tested through refinement of the mesh size, the size of the mesh override region, and the mesh accuracy as well as the conformal-mesh technology type (CMT type 1 was used). Geometric parameters of the silver nanocubes were modified to be most consistent with experimental observations; nanocubes with a corner roundness of $20 \%$ their edge length were found to be most consistent with similar sized cubes produced experimentally. Material fit parameters (number of coefficients and range) were tested, with parameters chosen which either minimised error or were most consistent with observed experimental results.

Three-dimensional visualizations of $|E|^{2}$ and charge density $(\rho)$ were obtained using Paraview (Kitware Inc.). Charge density was calculated from the divergence current.

\subsubsection{Experimental Methods}

Chemicals. Silver nitrate (AgNO3, 99\%+), polyvinylpyrrolidone (PVP, Mw $\sim 55000$ ), polystyrene (PS, Mw 192 000), anhydrous 99.8\% ethylene glycol (EG), sodium sulfide, ), 4-Mercaptobenzoicacid (MBA), Tetrakis(dimethylamido)zirconium(IV) (TDMAZr, Zr(NMe $\left.)_{4}\right)$, titanium(IV) isopropoxide (TTIP, Ti(iPrO)4), Trimethylaluminum (TMA, $\mathrm{AlMe}_{3}$,) toluene, and chloroform were purchased from Sigma-Aldrich and used as obtained. Copper nitrate (99\%). Sodium chloride (99\%+), DL-lactic acid (LA, 85-90\%), and sodium hydroxide (99\%) were obtained from Bioshop hydrazine (25\%) was obtained from Fluka. Ethanol (95\%) was obtained from Commercial Alcohols and used without further purification. 1,2-dioleoyl-sn-glycero-3-phosphocholine (DOPC) was purchased from Avanti Polar Lipids as a powder and used as obtained.

Preparation of Nanocubes. Silver nanocubes were synthesized according to the procedure described in the literature. ${ }^{78}$ To obtain larger cubes, $25 \mu \mathrm{L}$ of $0.48 \mathrm{M}$ sodium chloride in EG was added to the reaction flask prior to the injection of silver nitrate. When the characteristic peak found at $\sim 350 \mathrm{~nm}$ was developed an additional $1.5 \mathrm{~mL}$ of $282 \mathrm{mM}$ silver nitrate was added at a rate of $0.75 \mathrm{~mL} / \mathrm{min}$. The reaction was thermally quenched when the desired spectrum was observed. 
Preparation of Polystyrene Thin Films. Polystyrene was dissolved in toluene to produce $1 \%$ or $0.5 \%$ w/w solutions which were then filtered through a $0.220 \mu \mathrm{m}$ PTFE filter. Thin films were produced via static spin-coating on a Laurell WS-400-6NPP/LITE spin-coater. Films were allowed to anneal at $130^{\circ} \mathrm{C}$ overnight.

Preparation of AgNC Monolayers. Nanocube substrates were prepared by forming Langmuir films on a NIMA 311D trough filled with Milli-Q water $(18.2 \mathrm{M} \Omega \cdot \mathrm{cm})$. An appropriate amount of AgNC and DOPC was dispersed in chloroform and deposited onto the surface of the water using a microsyringe. Each monolayer was left for $20 \mathrm{~min}$ to allow complete chloroform evaporation. The surface pressure of the monolayer was measured with a paper Wilhelmy plate. Before transferring the monolayer onto solid supports several isotherm cycles were performed to anneal the films. Monolayers were transferred onto clean PS coated substrates at pressures corresponding to the gas phase of the isotherm via the Langmuir-Schaeffer deposition method.

Heating of Supported Monolayers of PS Films. A small steel block connected to a thermocouple was placed on top of a hotplate and used to heat substrates above $110{ }^{\circ} \mathrm{C}$ for specific intervals. Substrates were periodic imaged via AFM and UV-Visible spectroscopy.

Atomic Layer Deposition of $\mathrm{Al}_{2} \mathrm{O}_{3}, \mathrm{ZrO}_{2}, \mathrm{TiO}_{2}$. Thin film deposition on silver nanocube monolayers were conducted in a home built ALD tool. Briefly, the tool consisted of two heated precursor lines connected to a tube furnace. The $\mathrm{N}_{2}$ MFCs for each precursor line were set to $100 \mathrm{sccm}$, which set the tool pressure to 1 Torr. $\mathrm{AlMe}_{3}, \mathrm{Ti}(\mathrm{iPrO})_{4}$, and $\mathrm{Zr}\left(\mathrm{NMe}_{2}\right)_{4}$ were used as the metal precursors for thin films of $\mathrm{Al}_{2} \mathrm{O}_{3}, \mathrm{TiO}_{2}$, and $\mathrm{ZrO}_{2}$, respectively. $\mathrm{H}_{2} \mathrm{O}$ was used as the oxygen precursor. TTIP and TDMAZr were heated to $75{ }^{\circ} \mathrm{C}$ during the process and TMA was kept at room temperature. The tube furnace was heated to $100{ }^{\circ} \mathrm{C}$ for the deposition of $\mathrm{Al}_{2} \mathrm{O}_{3}$ and to $200{ }^{\circ} \mathrm{C}$ for the deposition of $\mathrm{TiO}_{2}$ and $\mathrm{ZrO}_{2}$. TMA and $\mathrm{H}_{2} \mathrm{O}$ were delivered with a $0.1 \mathrm{~s}$ pulse while $1 \mathrm{~s}$ pulses were used for TTIP and TDMAZr. Purge times were $20 \mathrm{~s}$ after each precursor.

The nanocube monolayers were prepared immediately prior to thin film deposition or stored under $\mathrm{N}_{2}$ in a desiccator until use. Nanocube monolayers treated with a SERS label (4-MBA) were prepared immediately prior to ALD. The pre-treatment for the monolayer samples was heating the samples at $100{ }^{\circ} \mathrm{C}$ for 30 min under a flow of $\mathrm{N}_{2}$. Growth rates for each metal oxide were measured 
by TEM. As synthesized nanocubes were drop-casted onto TEM grids and analysed after deposition of a uniform shell. Growth rates for $\mathrm{Al}_{2} \mathrm{O}_{3}, \mathrm{TiO}_{2}$, and $\mathrm{ZrO}_{2}$ were $1.4,0.3$, and $1.1 \AA$ /cycle, respectively.

Growth of $\mathrm{Cu}_{2} \mathrm{O}$ half-shells. For the growth of $\mathrm{Cu}_{2} \mathrm{O}$ half-shells on AgNCs, a sample slide of $1 \mathrm{~cm} \times 1 \mathrm{~cm}$ dimensions was attached to the end face of a glass rod, which was submerged and spun vigorously in $250 \mathrm{~mL}$ of $\mathrm{N}_{2}$ purged deionized water. Under constant flow on nitrogen, varied amounts of $5 \mathrm{M} \mathrm{NaOH}, 4 \mathrm{M}$ lactic acid, $0.5 \mathrm{M} \mathrm{Cu}\left(\mathrm{NO}_{3}\right)_{2}$, and hydrazine were added with differing order of addition. The reaction was left for either 50 or 55 minutes, dependent on the method of injection used. Once the reaction was complete, the sample slide was rinsed with $\mathrm{dH}_{2} \mathrm{O}$, then ethanol and dried under nitrogen. The coated nanocubes were removed from the substrate by sonicating the glass slide in a minimal amount of toluene to dissolve the polymer film.

\subsubsection{Characterization}

UV-Visible Measurements. Extinction spectra were obtained using a Cary 7000 equipped with a universal measurement analyser. Spectra were processed and analysed using OriginLab data analysis software.

Angle and polarization dependent transmittance and reflectance spectra were measured in $5^{\circ}$ increments for $s$ and $p$ polarized light using Cary 7000 UMS spectrophotometer. Absorptance spectra were calculated assuming no loss of light in the glass substrates.

Topographical Measurements. The topography of the NC monolayers transferred onto solid substrates was obtained using an Ntegra (NT-MDT, Russia) atomic force microscope in semicontact mode in air at $21^{\circ} \mathrm{C}$ with $512 \times 512$ or $1024 \times 1024$ points per image. A $100 \times 100 \mu m^{2}$ scanner (Ntegra) and cantilevers with rotated monolithic silicon tips (135 $\mu \mathrm{m}$ long, 0.3-6 N/m spring constant NSG03, resonance frequency $90 \mathrm{kHz}$, NT-MDT) were used for all topographic measurements. The typical scan rate was $0.5 \mathrm{~Hz}$. AFM images were further processed by Nova image processing software. 
Raman Spectroscopy. For SERS the silver nanocube monolayer was incubated in a $5 \mathrm{mM}$ solution of MBA in anhydrous ethanol for 1 hour. The slide was then rinsed with anhydrous ethanol and annealed at $100{ }^{\circ} \mathrm{C}$ for 30 min under a flow of $\mathrm{N}_{2}$ prior to characterization.

Raman measurements were performed using an $\mathrm{Ar} / \mathrm{Kr}$ ion laser (Coherent) at 458, 488, 514, 568, and $647 \mathrm{~nm}$ and passed through an inverted microscope (Olympus IX-71) with a 20x objective for excitation. The SERS signal was collected through the same objective in a backscattering geometry. Raman spectra were collected using a single grating monochromator (Jobin Yvon, 640 $\mathrm{mm}$ focal length, 1200 lines $/ \mathrm{mm}$ ) with a liquid $\mathrm{N}_{2}$ cooled CCD camera (Princeton Instruments)

\subsection{References}

(1) Noguez, C. Surface Plasmons on Metal Nanoparticles: The Influence of Shape and Physical Environment. J. Phys. Chem. C 2007, 111 (10), 3806-3819.

(2) Gallinet, B.; Siegfried, T.; Sigg, H.; Nordlander, P.; Martin, O. J. F. Plasmonic Radiance: Probing Structure at the Ångström Scale with Visible Light. Nano Lett. 2013, 13 (2), 497503.

(3) Yun, C. S.; Javier, A.; Jennings, T.; Fisher, M.; Hira, S.; Peterson, S.; Hopkins, B.; Reich, N. O.; Strouse, G. F. Nanometal Surface Energy Transfer in Optical Rulers, Breaking the FRET Barrier. J. Am. Chem. Soc. 2005, 127 (9), 3115-3119.

(4) Shen, S.; Meng, L.; Zhang, Y.; Han, J.; Ma, Z.; Hu, S.; He, Y.; Li, J.; Ren, B.; Shih, T. M.; Wang, Z.; Yang, Z.; Tian, Z. Plasmon-Enhanced Second-Harmonic Generation Nanorulers with Ultrahigh Sensitivities. Nano Lett. 2015, 15 (10), 6716-6721.

(5) Moreau, A.; Ciracì, C.; Mock, J. J.; Hill, R. T.; Wang, Q.; Wiley, B. J.; Chilkoti, A.; Smith, D. R. Controlled-Reflectance Surfaces with Film-Coupled Colloidal Nanoantennas. Nature 2012, 492 (7427), 86-89.

(6) Hill, R. T.; Kozek, K. M.; Hucknall, A.; Smith, D. R.; Chilkoti, A. Nanoparticle-Film Plasmon Ruler Interrogated with Transmission Visible Spectroscopy. ACS Photonics 2014, 1 (10), 974-984.

(7) Kovacs, G. J.; Vincett, P. S. Formation and Thermodynamic Stability of a Novel Class of Useful Materials: Close-Packed Monolayers of Submicron Monodisperse Spheres Just below a Polymer Surface. J. Colloid Interface Sci. 1982, 90 (2), 335-351.

(8) Deshmukh, R. D.; Composto, R. J. Direct Observation of Nanoparticle Embedding into the Surface of a Polymer Melt. Langmuir 2007, 23 (26), 13169-13173.

(9) Erichsen, J.; Kanzow, J.; Schürmann, U.; Dolgner, K.; Günther-Schade, K.; Strunskus, T.; Zaporojtchenko, V.; Faupel, F. Investigation of the Surface Glass Transition Temperature by Embedding of Noble Metal Nanoclusters into Monodisperse Polystyrenes. Macromolecules 2004, 37 (5), 1831-1838.

(10) Butet, J.; Martin, O. J. F. Nonlinear Plasmonic Nanorulers. ACS Nano 2014, 8 (5), 49314939.

(11) Gallinet, B.; Siegfried, T.; Sigg, H.; Nordlander, P.; Martin, O. J. F. Plasmonic Radiance: Probing Structure at the Ångström Scale with Visible Light. Nano Lett. 2013, 13 (2), 497- 
503.

(12) Jain, P. K.; Huang, W.; El-Sayed, M. A. On the Universal Scaling Behavior of the Distance Decay of Plasmon Coupling in Metal Nanoparticle Pairs: A Plasmon Ruler Equation. Nano Lett. 2007, 7 (7), 2080-2088.

(13) Liu, N.; Hentschel, M.; Weiss, T.; Alivisatos, a P.; Giessen, H. Three-Dimensional Plasmon Rulers. Science (80-. ). 2011, 332 (6036), 1407-1410.

(14) Singh, A. K.; Khan, S. A.; Fan, Z.; Demeritte, T.; Senapati, D.; Kanchanapally, R.; Ray, P. C. Development of a Long-Range Surface-Enhanced Raman Spectroscopy Ruler. J. Am. Chem. Soc. 2012, 134 (20), 8662-8669.

(15) Breshike, C. J.; Riskowski, R. A.; Strouse, G. F. Leaving Förster Resonance Energy Transfer behind: Nanometal Surface Energy Transfer Predicts the Size-Enhanced Energy Coupling between a Metal Nanoparticle and an Emitting Dipole. J. Phys. Chem. C 2013, 117 (45), 23942-23949.

(16) Bourret, G. R.; Ozel, T.; Blaber, M.; Shade, C. M.; Schatz, G. C.; Mirkin, C. A. LongRange Plasmophore Rulers. Nano Lett. 2013, 13 (5), 2270-2275.

(17) Li, L.; Hutter, T.; Li, W.; Mahajan, S. Single Nanoparticle-Based Heteronanojunction as a Plasmon Ruler for Measuring Dielectric Thin Films. J. Phys. Chem. Lett. 2015, 6 (12), 2282-2286.

(18) Zhang, S.; Bao, K.; Halas, N. J.; Xu, H.; Nordlander, P. Substrate-Induced Fano Resonances of a Plasmonic Nanocube: A Route to Increased-Sensitivity Localized Surface Plasmon Resonance Sensors Revealed. Nano Lett. 2011, 11 (4), 1657-1663.

(19) Ahamad, N.; Bottomley, A.; lanoul, A. Optimizing Refractive Index Sensitivity of Supported Silver Nanocube Monolayers. J. Phys. Chem. C 2012, 116 (1), 185-192.

(20) Sherry, L. J.; Chang, S.-H.; Schatz, G. C.; Van Duyne, R. P.; Wiley, B. J.; Xia, Y. Localized Surface Plasmon Resonance Spectroscopy of Single Silver Nanocubes. Nano Lett. 2005, 5 (10), 2034-2038.

(21) Zhang, Q.; Li, W.; Moran, C.; Zeng, J.; Chen, J.; Wen, L.-P.; Xia, Y. Seed-Mediated Synthesis of Ag Nanocubes with Controllable Edge Lengths in the Range of 30-200 Nm and Comparison of Their Optical Properties. J. Am. Chem. Soc. 2010, 132 (32), 1137211378.

(22) Ringe, E.; McMahon, J. M.; Sohn, K.; Cobley, C.; Xia, Y.; Huang, J.; Schatz, G. C.; Marks, L. D.; Van Duyne, R. P. Unraveling the Effects of Size, Composition, and Substrate on the Localized Surface Plasmon Resonance Frequencies of Gold and Silver Nanocubes: A Systematic Single-Particle Approach. J. Phys. Chem. C 2010, 114 (29), 12511-12516.

(23) Nicoletti, O.; de la Peña, F.; Leary, R. K.; Holland, D. J.; Ducati, C.; Midgley, P. a. ThreeDimensional Imaging of Localized Surface Plasmon Resonances of Metal Nanoparticles. Nature 2013, 502 (7469), 80-84.

(24) Edwards, P. R.; Sleith, D.; Wark, A. W.; Martin, R. W. Mapping Localized Surface Plasmons within Silver Nanocubes Using Cathodoluminescence Hyperspectral Imaging. J. Phys. Chem. C 2011, 115 (29), 14031-14035.

(25) Rieger, J. The Glass Transition Temperature of Polystyrene. J. Therm. Anal. 1996, 46 (34), 965-972.

(26) Vignaud, G.; Chebil, M. S.; Bal, J. K.; Delorme, N.; Beuvier, T.; Grohens, Y.; Gibaud, A. Densification and Depression in Glass Transition Temperature in Polystyrene Thin Films. Langmuir 2014, 30 (39), 11599-11608.

(27) Bottomley, A.; Prezgot, D.; Coyle, J. P.; lanoul, A. Dynamics of Nanocubes Embedding into Polymer Films Investigated via Spatially Resolved Plasmon Modes. Nanoscale 2016, 8 (21), 11168-11176. 
(28) Yang, H. U.; D’Archangel, J.; Sundheimer, M. L.; Tucker, E.; Boreman, G. D.; Raschke, M. B. Optical Dielectric Function of Silver. Phys. Rev. B 2015, 91 (23), 235137.

(29) Higashihara, T.; Ueda, M. Recent Progress in High Refractive Index Polymers. Macromolecules 2015, 48 (7), 1915-1929.

(30) Kelly, K.; Coronado, E. The Optical Properties of Metal Nanoparticles: The Influence of Size, Shape, and Dielectric Environment. J. Phys. Chem. B 2003, 107, 668-677.

(31) Mulvaney, P. Surface Plasmon Spectroscopy of Nanosized Metal Particles. Langmuir 1996, 12 (3), 788-800.

(32) Knight, M. W.; Wu, Y.; Lassiter, J. B.; Nordlander, P.; Halas, N. J. Substrates Matter: Influence of an Adjacent Dielectric on an Individual Plasmonic Nanoparticle. Nano Lett. 2009, 9 (5), 2188-2192.

(33) Bottomley, A.; Prezgot, D.; Staff, A.; lanoul, A. Fine Tuning of Plasmonic Properties of Monolayers of Weakly Interacting Silver Nanocubes on Thin Silicon Films. Nanoscale 2012, 4 (20), 6374-6382.

(34) Prezgot, D.; lanoul, A. Probing the Anisotropy of SERS Enhancement with Spatially Separated Plasmonic Modes in Strongly Coupled Silver Nanocubes on a Dielectric Substrate. J. Phys. Chem. C 2015, 119 (6), 3293-3301.

(35) Bottomley, A.; lanoul, A. Reflection and Absorption Spectra of Silver Nanocubes on a Dielectric Substrate: Anisotropy, Angle, and Polarization Dependencies. J. Phys. Chem. C 2014, 118 (47), 27509-27515.

(36) Saito, K.; Tanabe, I.; Tatsuma, T. Site-Selective Plasmonic Etching of Silver Nanocubes. J. Phys. Chem. Lett. 2016, 7 (21), 4363-4368.

(37) Fang, C.; Lee, Y. H.; Shao, L.; Jiang, R.; Wang, J.; Xu, Q. H. Correlating the Plasmonic and Structural Evolutions during the Sulfidation of Silver Nanocubes. ACS Nano 2013, 7 (10), 9354-9365.

(38) Kumar, P.; Wiedmann, M. K.; Winter, C. H.; Avrutsky, I. Optical Properties of Al2O3thin Films Grown by Atomic Layer Deposition. Appl. Opt. 2009, 48 (28), 5407-5412.

(39) Munechika, K.; Smith, J. M.; Chen, Y.; Ginger, D. S. Plasmon Line Widths of Single Silver Nanoprisms as a Function of Particle Size and Plasmon Peak Position. J. Phys. Chem. C 2007, 111 (51), 18906-18911.

(40) Hu, M.; Petrova, H.; Sekkinen, A. R.; Chen, J.; McLellan, J. M.; Li, Z. Y.; Marquez, M.; Li, X.; Xia, Y.; Hartland, G. V. Optical Properties of Au-Ag Nanoboxes Studied by Single Nanoparticle Spectroscopy. J. Phys. Chem. B 2006, 110 (40), 19923-19928.

(41) Sönnichsen, C.; Franzl, T.; Wilk, T.; Von Plessen, G.; Feldmann, J. Plasmon Resonances in Large Noble-Metal Clusters. New J. Phys. 2002, 4.

(42) Kravets, V. G.; Schedin, F.; Grigorenko, A. N. Extremely Narrow Plasmon Resonances Based on Diffraction Coupling of Localized Plasmons in Arrays of Metallic Nanoparticles. Phys. Rev. Lett. 2008, 101 (8), 1-4.

(43) Auguié, B.; Barnes, W. L. Collective Resonances in Gold Nanoparticle Arrays. Phys. Rev. Lett. 2008, 101 (14), 1-4.

(44) Malynych, S.; Chumanov, G. Light-Induced Coherent Interactions between Silver Nanoparticles in Two-Dimensional Arrays. J. Am. Chem. Soc. 2003, 125 (10), 2896-2898.

(45) Taubert, R.; Hentschel, M.; Kästel, J.; Giessen, H. Classical Analog of Electromagnetically Induced Absorption in Plasmonics. Nano Lett. 2012, 12 (3), 1367-1371.

(46) Zeuner, F.; Muldarisnur, M.; Hildebrandt, A.; Förstner, J.; Zentgraf, T. Coupling Mediated Coherent Control of Localized Surface Plasmon Polaritons. Nano Lett. 2015, 15 (6), 
4189-4193.

(47) Yang, Z. J. Coherent Energy Transfers between Orthogonal Radiant and Weakly Radiant Plasmonic Nanorod Resonators. J. Phys. Chem. C 2015, 119 (46), 26079-26085.

(48) Hein, S. M.; Giessen, H. Retardation-Induced Phase Singularities in Coupled Plasmonic Oscillators. Phys. Rev. B - Condens. Matter Mater. Phys. 2015, 91 (20), 1-6.

(49) Svedendahl, M.; Johansson, P.; Käll, M. Complete Light Annihilation in an Ultrathin Layer of Gold Nanoparticles. Nano Lett. 2013, 13 (7), 3053-3058.

(50) Howse, J. R.; Jones, R. A. L.; Ryan, A. J.; Gough, T.; Vafabakhsh, R.; Golestanian, R. Self-Motile Colloidal Particles: From Directed Propulsion to Random Walk. Phys. Rev. Lett. 2007, 99, 048102.

(51) Yoshida, M.; Roh, K. H.; Lahann, J. Short-Term Biocompatibility of Biphasic Nanocolloids with Potential Use as Anisotropic Imaging Probes. Biomaterials 2007, 28 (15), 2446-2456.

(52) McConnell, M. D.; Kraeutler, M. J.; Yang, S.; Composto, R. J. Patchy and Multiregion Janus Particles with Tunable Optical Properties. Nano Lett. 2010, 10 (2), 603-609.

(53) Glotzer, S. C.; Solomon, M. J. Anisotropy of Building Blocks and Their Assembly into Complex Structures. Nat. Mater. 2007, 6 (8), 557-562.

(54) Panayotov, D. A.; Frenkel, A. I.; Morris, J. R. Catalysis and Photocatalysis by Nanoscale Au/TiO2: Perspectives for Renewable Energy. ACS Energy Lett. 2017, 2 (5), 1223-1231.

(55) Wu, N. Plasmonic Metal-Semiconductor Photocatalysts and Photoelectrochemical Cells: A Review. Nanoscale 2018, 10 (6), 2679-2696.

(56) Li, J.; Cushing, S. K.; Bright, J.; Meng, F.; Senty, T. R.; Zheng, P.; Bristow, A. D.; Wu, N. Ag@Cu2O Core-Shell Nanoparticles as Visible-Light Plasmonic Photocatalysts. ACS Catal. 2013, 3 (1), 47-51.

(57) Li, J.; Cushing, S. K.; Zheng, P.; Meng, F.; Chu, D.; Wu, N. Plasmon-Induced Photonic and Energy-Transfer Enhancement of Solar Water Splitting by a Hematite Nanorod Array. Nat. Commun. 2013, 4, 1-8.

(58) Chen, T.; Yang, M.; Wang, X.; Li, H. T.; Chen, H. Controlled Assembly of Eccentrically Encapsulated Gold Nanoparticles. J. Am. Chem. Soc. 2008, 130 (36), 11858-11859.

(59) Jia, H.; Du, A.; Zhang, H.; Yang, J.; Jiang, R.; Wang, J.; Zhang, C. Y. Site-Selective Growth of Crystalline Ceria with Oxygen Vacancies on Gold Nanocrystals for NearInfrared Nitrogen Photofixation. J. Am. Chem. Soc. 2019, 141 (13), 5083-5086.

(60) Chen, T.; Chen, G.; Xing, S.; Wu, T.; Chen, H. Scalable Routes to Janus Au-SiO2 and Ternary Ag-Au-SiO2. Chem. Mater. 2010, 22, 3826-3828.

(61) Crane, C. C.; Tao, J.; Wang, F.; Zhu, Y.; Chen, J. Mask-Assisted Seeded Growth of Segmented Metallic Heteronanostructures. J. Phys. Chem. C 2014, 118, 28134-28142.

(62) Pothorszky, S.; Zámbó, D.; Deák, A. Structural and Optical Properties of Gold/Silica "Mushroom" Particles Prepared by Interfacial Templating. Part. Part. Syst. Charact. 2017, 34, 1600291.

(63) Miyanohata, R.; Matsushita, T.; Tsuruoka, T.; Nawafune, H.; Akamatsu, K. A Facile Template Synthesis of Asymmetric Gold Silica Heteronanoparticles. J. Colloid Interface Sci. 2014, 416, 147-150.

(64) Jimenez-Cadena, G.; Comini, E.; Ferroni, M.; Sberveglieri, G. Synthesis of Cu2O BiPyramids by Reduction of $\mathrm{Cu}(\mathrm{OH}) 2$ in Solution. Mater. Lett. 2010, 64 (3), 469-471.

(65) Jing, H.; Large, N.; Zhang, Q.; Wang, H. Epitaxial Growth of Cu2O on Ag Allows for Fine Control over Particle Geometries and Optical Properties of Ag-Cu2O Core-Shell Nanoparticles. J. Phys. Chem. C 2014, 118 (34), 19948-19963. 
(66) Kuo, C.-H.; Hua, T.-E.; Huang, M. H. Au Nanocrystal-Directed Growth of Au-Cu2O Core-Shell Heterostructures with Precise Morphological Control. J. Am. Chem. Soc. 2009, 131 (49), 17871-17878.

(67) Sun, Y.; Xia, Y. Shape-Controlled Synthesis of Gold and Silver Nanoparticles. Science (80-. ). 2002, 298 (5601), 2176-2179.

(68) Kuo, C.; Huang, M. H. Morphologically Controlled Synthesis of $\mathrm{Cu}_{2} \mathrm{O}$ Nanocrystals and Their Properties. Nano Today 2010, 5 (2), 106-116.

(69) Wang, Z.; Wang, H.; Wang, L.; Pan, L. Controlled Synthesis of $\mathrm{Cu}_{2} \mathrm{O}$ Cubic and Octahedral Nano-and Microcrystals. Cryst. Res. Technol. 2009, 44 (6), 624-628.

(70) Sciacca, B.; Mann, S. A.; Tichelaar, F. D.; Zandbergen, H. W.; Van Huis, M. A.; Garnett, E. C. Solution-Phase Epitaxial Growth of Quasi-Monocrystalline Cuprous Oxide on Metal Nanowires. Nano Lett. 2014, 14 (10), 5891-5898.

(71) Kong, L.; Chen, W.; Ma, D.; Yang, Y.; Liu, S.; Huang, S. Size Control of Au@Cu 20 Octahedra for Excellent Photocatalytic Performance. J. Mater. Chem. 2012, 22 (2), 719724.

(72) Ho, J.; Huang, M. H. Synthesis of Submicrometer-Sized Cu 2 O Crystals with Morphological Evolution from Cubic to Hexapod Structures and Their Comparative Photocatalytic Activity. J. Phys. Chem. 2009, 113, 14159-14164.

(73) Palik, E. D. Handbook of Optical Constants of Solids; 1985; Vol. 1.

(74) Kim, H.; Lee, M. Y.; Kim, S.-H.; Bae, S. I.; Ko, K. Y.; Kim, H.; Kwon, K.-W.; Hwang, J.-H.; Lee, D.-J. Highly-Conformal p-Type Copper(I) Oxide (Cu2O) Thin Films by Atomic Layer Deposition Using a Fluorine-Free Amino-Alkoxide Precursor. Appl. Surf. Sci. 2015, 349, 673-682.

(75) Yu, W.; Mittra, R. A Conformal Finite Difference Time Domain Technique for Modeling Curved Dielectric Surfaces. IEEE Microw. Wirel. Components Lett. 2001, 11 (1), 25-27.

(76) Sultanova, N.; Kasarova, S.; Nikolov, I. Dispersion Properties of Optical Polymers. Acta Phys. Pol. A 2009, 116 (4), 585-587.

(77) König, T. A. F.; Ledin, P. A.; Kerszulis, J.; Mahmoud, M. A.; El-Sayed, M. A.; Reynolds, J. R.; Tsukruk, V. V. Electrically Tunable Plasmonic Behavior of Nanocube-Polymer Nanomaterials Induced by a Redox-Active Electrochromic Polymer. ACS Nano 2014, 8 (6), 6182-6192.

(78) Skrabalak, S. E.; Au, L.; Li, X.; Xia, Y. Facile Synthesis of Ag Nanocubes and Au Nanocages. Nat. Protoc. 2007, 2 (9), 2182-2190. 


\section{Chapter 3: Thermoplasmonic patterning of silver nanocrystal/polymer composite thin films}
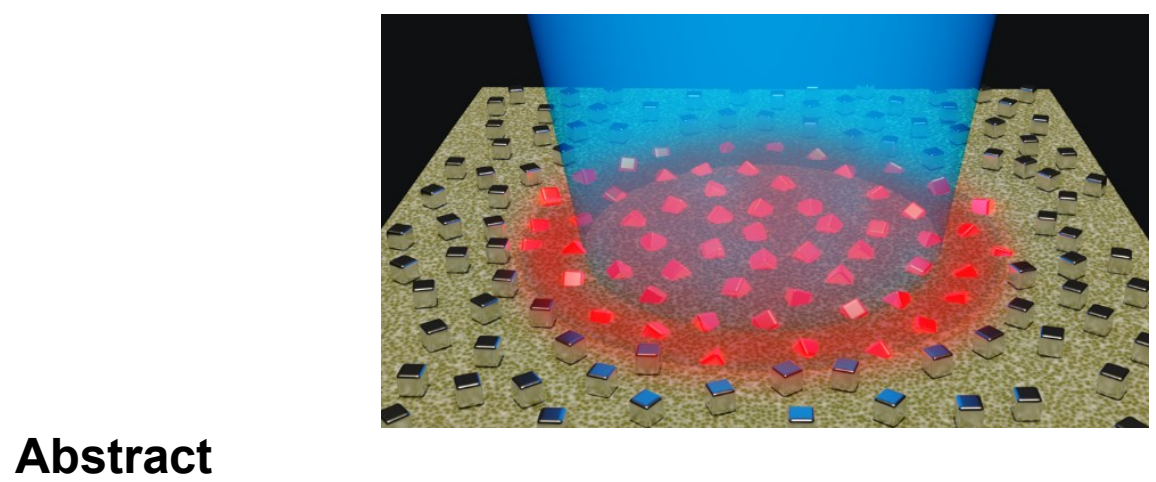

The lithographic patterning of polymer films is ubiquitous in manufacturing processes. In this work we utilize the inherently high photothermal conversion efficiency of plasmonic nanoparticles to locally pattern polystyrene films. Silver nanocubes were deposited onto polystyrene thin films and were used as a heat source for photothermal lithographic patterning using a continuous-wave, visible laser. Distinct states defined by their own far-field optical properties and micro-scale topography are observed. Using low intensities $\left(>0.086 \mathrm{~mW} / \mu \mathrm{m}^{2} @\right.$ $458 \mathrm{~nm}$ ) the nanocubes can be embedded into the polymer at a nanometer scale displacement and produced a notable shift in the distinct localized surface plasmon resonance (LSPR) of silver nanocubes, enabling two-color optical patterning of the film. At higher laser intensities $>0.434$ $\mathrm{mW} / \mu \mathrm{m}^{2}$ ) sufficient temperatures were reached for the complete photothermal ablation of the polymer film. Temperatures in the film easily surpass the onset of photothermal decomposition (> $270^{\circ} \mathrm{C}$ ), and the decomposition process is thermal in nature. Temperature estimates also highlight the role of the collective contribution of nanoparticle heating in the process. The process can be controlled by tuning the power absorbed by the nanocubes in accordance with their plasmonic absorption spectrum. The approach here outlines a general method of 2D optical patterning utilizing thermoplasmonics which can be further expanded to other materials, applications and potentially to sub-diffraction length scales.

Prezgot, D.; Bottomley, A.; Jorgenson, E.; lanoul, A. Thermoplasmonic patterning of silver nanocrystal/polymer composite thin films, Manuscript in preparation 


\subsection{Introduction}

Noble metal nanostructures enable the manipulation of electromagnetic radiation down to nanoscale dimensions due to their ability to scatter and absorb light through the localised surface plasmon resonance (LSPR). The light absorbed by metal nanoparticles is rapidly converted to heat as the excited hot-electrons relax through electron-phonon interactions on the order of picosends. ${ }^{1}$ The high thermal conductivity of the nanoparticles enables them to quickly dissipate this heat to their surroundings; they reach steady-state temperatures with their environment within nanoseconds. ${ }^{2,3}$ This is known as the thermoplasmonic effect which enables the rapid pinpoint delivery of heat and has seen use in areas such as photothermal therapeutics, ${ }^{4,5}$ photo-catalysis, ${ }^{6,7}$ and solar thermal energy harvesting. ${ }^{8,9}$ Similarly, the use of metal nanoparticles as thermoplasmonic heat sources has been applied to dynamic optical colour patterning. ${ }^{10,11}$ This can be achieved by altering the shape, ${ }^{10}$ or local environment of the particles in order to alter their LSPR. ${ }^{12}$ Plasmonic-heat sources can also be utilized in micro/nanofabrication for mask-less directwrite optical lithography, bypassing the need for the creation of complex masks, or expensive electron or ion-beam lithography to achieve high resolution patterning. Plasmonic heat sources generate highly-localized heating which can be applied from sub-diffraction limited scales to microscale or larger patterning. ${ }^{13-15}$

Our recent progress in understanding properties of supported plasmonic nanocrystals allows us to better manipulate their LSPR signatures control them using the thermoplasmonic effect. ${ }^{16,17}$ Here we use the LSPR of silver nanocubes to collect light and locally deliver heat to a polymer film, changing the physical properties of the polymer to produce microscale optical patterning. The large surface contact afforded by the geometry of silver nanocubes leads to distinct and sensitive spatially separated hybridized plasmon modes, ${ }^{18}$ in addition to more efficient heat transfer to the substrate. Metal nanoparticles on the polymer surface can be selectively embedded into the polymer upon photothermal heating, ${ }^{13}$ thereby altering their local environment and LSPR signature. Here we demonstrate that by controlling the amount of power absorbed by the silver nanocubes, the resultant changes in the polymer film can be finely tuned. Effects such as significant LSPR shifts, microscopic topographical changes in the polymer, and even photothermal ablation 
of the polymer film are observed at modest intensities using a continuous-wave $(\mathrm{CW})$ visible light laser.

\subsection{Experimental Methods}

Materials: Silver nitrate $\left(\mathrm{AgNO}_{3}, 99+\%\right)$, polyvinylpyrrolidone (PVP, $\mathrm{MW} \sim 55,000$ ), anhydrous ethylene glycol (EG, 99.8\%), sodium sulfide $\left(\mathrm{Na}_{2} \mathrm{~S}\right)$, and sodium chloride $(\mathrm{NaCl})$ were purchased from Sigma-Aldrich and used as obtained. Polystyrene beads (PS, MW 250000 ) were obtained from Acros Organics.

Synthesis of silver nanocubes: Silver nanocubes were synthesized in a one-step polyol technique. $15 \mathrm{~mL}$ of EG was preheated to $150^{\circ} \mathrm{C}$ in a $25 \mathrm{~mL}$ round-bottom flask. $2.5 \mathrm{~mL}$ of $0.8 \mathrm{mg} / \mathrm{mL}$ PVP, $200 \mu \mathrm{L}$ of $3 \mathrm{mM} \mathrm{Na} 2 \mathrm{~S}, 25 \mu \mathrm{L}$ of $0.45 \mathrm{M} \mathrm{NaCl}$ was subsequently injected into the flask. $1.5 \mathrm{~mL}$ of $282 \mathrm{mM} \mathrm{AgNO}_{3}$ was added to the flask at a flow rate of $0.5 \mathrm{~mL} / \mathrm{min}$ via a syringe pump. The reaction was monitored by UV-Vis spectroscopy of aliquots. After $\sim 1 \mathrm{~h}$ the sample showed signs of growth of the nanocrystals (indicated by a red-shifting of the plasmon) and was thermally quenched upon reaching the desired spectral signature.

Preparation of substrates: A polystyrene solution ( $1 \% \mathrm{w} / \mathrm{w}$ in toluene) were prepared and then passed through a $0.22 \mu \mathrm{m}$ PTFE filter using a glass syringe. Thin films were produced via static spin-coating onto glass slides at $\sim 4000 \mathrm{rpm}$ on a custom-built spin coater. Films were allowed to dry for $1 \mathrm{~h}$ then annealed for up to $12 \mathrm{~h}$ at $120^{\circ} \mathrm{C}$.

Silver nanocube monolayers were deposited onto the polystyrene films using the LangmuirSchaeffer technique using a NIMA 311D Langmuir trough filled with milli $Q$ water (1.8 $\mathrm{M} \Omega$ ). AgNCs were dispersed in $300 \mu \mathrm{L}$ of chloroform and deposited onto the water surface using a micro-syringe. Monolayers were transferred onto PS thin films in the gas phase of the isotherm at a surface pressure of $0.5 \mathrm{mN} / \mathrm{m}$.

Laser Patterning: An Ar/Kr ion laser (Coherent 170 series) was passed through either an achromatic doublet, or a 0.7 NA objective focused onto the substrate which was mounted on a six-axis stage. Excitation was performed at 458, 476, 514, 568 and $657 \mathrm{~nm}$. Power was measured by a power meter placed immediately before the focusing lens. The waist radius of the beam was determined using the knife-edge technique with a cleaved silicon wafer. To acquire UV-Vis spectra of the 
photothermally embedded area the beam was defocused, and the sample was manually raster scanned to produce a $2 \times 2 \mathrm{~mm}$ patterned area.

Characterization: Transmission and reflection spectra of the substrates before patterning were obtained using a Cary 7000 equipped with a universal measurement analyzer. Transmission and reflection spectra of raster scanned areas were obtained on an Ocean Optics USB-2000 optic fiber spectrometer with a xenon pulsed lamp.

The topographic measurements of the substrates before and after patterning were obtained using an Ntegra (NT-MDT) atomic force microscope in semi-contact mode. Gold-reflexed cantilevers with rotated monolithic silicon tips $(135 \mu \mathrm{m}$ long, $0.3-6 \mathrm{~N} / \mathrm{m}$ spring constant, resonance frequency $\sim 90$ kHz, NSG03, NT-MDT). Scans were performed at rates between $0.4 \mathrm{~Hz}$ to $0.7 \mathrm{~Hz}$ depending on the size and resolution of the image. AFM images were processed by Nova image processing software (NT-MDT).

Raman Spectroscopy: Surface enhanced Raman spectra were collected using a single grating monochromator (Jobin Yvon, focal length 640nm, 1600 lines $/ \mathrm{mm}$ ) with a liquid N2 cooled CCD camera (Princeton Instruments). Excitation was done using an $\mathrm{Ar} / \mathrm{Kr}$ ion laser (Coherent) at 488 $\mathrm{nm}$, focused through a $60 \mathrm{X}$ objective $(0.7 \mathrm{NA})$, and passed through 2 notch filters (Thorlabs). Cyclohexane was used for Raman shift calibration. Samples were submerged in $10^{-5} \mathrm{M}$ aqueous Rhodamine 6G (Rh6G) for $\sim 1 \mathrm{~h}$ before measurements.

Numerical Modelling: Finite-difference time-domain (FDTD) calculations were used to estimate the cross-section of silver nanocubes on a polystyrene substrate. Calculations were performed using a commercial solver (Lumerical FDTD Solutions, v8.16). The models consisted of silver nanocubes with an edge length between 45 and $65 \mathrm{~nm}$ with a corner radius of $20 \%$ their edge length and a 2 $\mathrm{nm}$ capping layer of PVP, supported on a polystyrene film. Materials were described by an analytical model fitted to tabulated permittivity data. ${ }^{19-21} \mathrm{~A}$ total-field scattered-field light source was used with perfect matched layer boundary conditions. 


\subsection{Results and Discussion}

\subsubsection{Thermoplasmonic patterning of silver nanocrystal-on-polymer films}

To investigate the efficiency and quantify the tunability of thermoplasmonic effect in this work we utilized a well-studied nanocomposite consisting of a monolayer of $55 \pm 10 \mathrm{~nm}$ silver nanocubes deposited on polystyrene thin films $(200 \pm 6 \mathrm{~nm})$ via Langmuir-Schaeffer method. ${ }^{17}$ The as deposited the nanocubes form a quasi-disordered 2D array with an average interparticle distance of $190 \mathrm{~nm}$ (Figure 3.1a), providing sufficient spacing to prevent interparticle plasmonic coupling so that the particles can be considered optically independent. ${ }^{22-24}$ The effect of neighboring particles is negligible which is corroborated by the absence of a strong coupling band in their extinction spectra (Figure 3.1b). Polystyrene was chosen as the polymer substrate due to its modest glass transition temperature $\left(\mathrm{T}_{\mathrm{g}} \sim 100{ }^{\circ} \mathrm{C}\right)$, and its favorable interaction with the silver nanocubes facilitating fabrication of the substrate and maximizing their embedment upon heating. ${ }^{25}$ Additionally, the polymer has a relatively high refractive index $(n \approx 1.6)$ which results in stronger silver nanocube plasmon mode hybridization and therefore a more pronounced LSPR shift upon embedment.
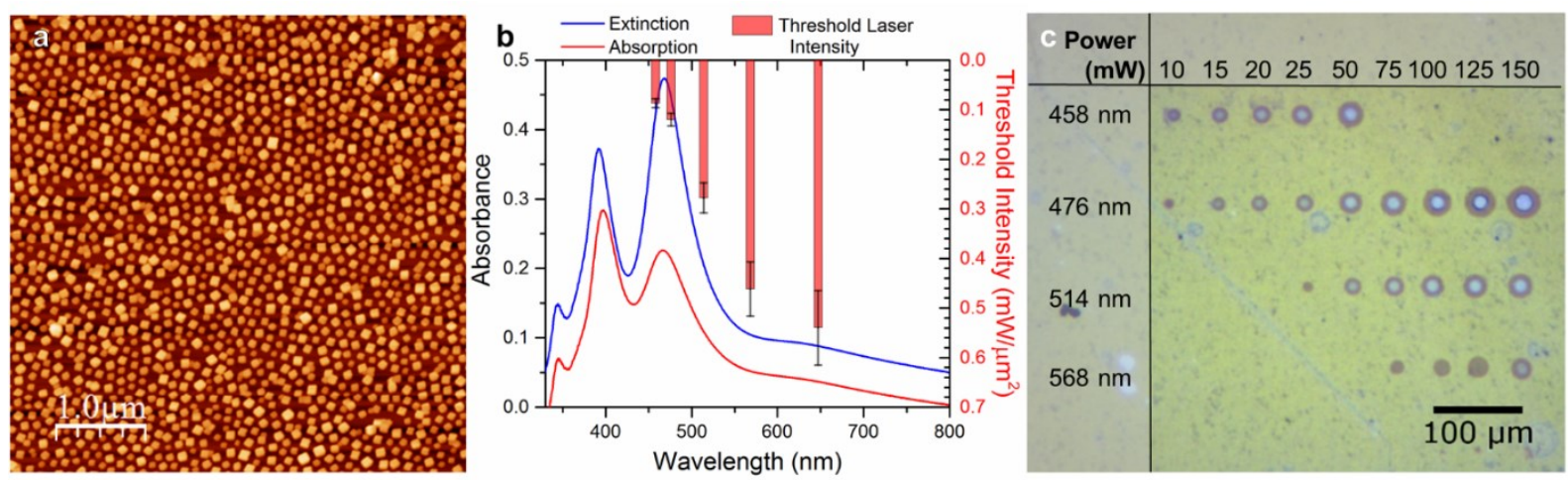

Figure 3.1. a) Atomic force microscopy (AFM) of the initial sample, displaying of $55 \pm 10 \mathrm{~nm}$ cubes with an average interparticle spacing of $\sim 190 \mathrm{~nm}$ b) UV/Vis extinction spectrum (blue) and the calculated absorption spectrum (red) of the target sample. The threshold peak intensity required for embedment (represented as bars) inversely follows the plasmon absorbance of the particles. c) Optical microscopy image of an area of the slide exposed to different powers and wavelengths of laser light for $10 \mathrm{~s}$. The resultant size and topography of the patterned spot can be controlled with these parameters.

To perform patterning of the substrate it was exposed to a continuous-wave laser focused by an achromatic lens at various incident powers at five fixed wavelengths ( $458 \mathrm{~nm}, 476 \mathrm{~nm}, 514$ $\mathrm{nm}, 568 \mathrm{~nm}$ and $647 \mathrm{~nm}$ ). Changes were visibly observed on the polymer surface after brief exposures ( $>1 \mathrm{~s}$ ) provided that the absorbed power exceeded a minimum threshold (Figure 
3.1b,c). The size and visual appearance of the features observed on the substrate were dependent on the exposure time, wavelength, and power used. With short exposure times $(\sim 1 \mathrm{~s})$, the radii of spots in the substrate are measured to be on the order of size as the waist radius of the beam $(\sim 10$ $\mu \mathrm{m})$. When the exposure was increased so was the resultant spot's diameter and a progressive change in morphology. It was found a certain threshold power was necessary to produce a pattern, below which even long exposures (up to $120 \mathrm{~s}$ ) failed to produce a change in the substrate (Figure D.2). This threshold power was found to depend on the incident wavelength of light and scaled in accordance with the absorbance of the LSPR as the heat generated scales with the power absorbed by the nanoparticle (Figure 3.1b). The absorbance $(A)$ is calculated from transmission $(T)$ and reflection $\left.(R), A=-\log _{10}[(1-T)-R)\right]$. Patterning occurred on a sufficiently small timescale that lines could be drawn into the polymer surface by scanning the beam across the substrate. Patterning was systematically performed across the visible spectrum at a range of incident powers with $10 \mathrm{~s}$ exposures. It was observed that the polymer surface generally fell into three reoccurring states after exposure (Figure 3.2):

I) Embedment of the nanocubes into the polymer surface with little change in morphology. (Figure 3.2 a- c) This stage could be rationalized by the local heating of the polymer film above the $T_{g}$ of the polymer film followed by the partial nanocube incorporation.

II) At this stage, the greater population of nanocubes are fully embedded into the supporting matrix and surface deformation of the polymer film is present, though the film is still continuous. (Figure $3.2 \mathrm{~d}$-f)The local temperature of the polymer at this stage exceeds the $T_{g}$.

III) Finally, at laser powers significantly above the threshold, decomposition, ablation and displacement of the polymer film, is observed leaving a hole in the film, along with a region of embedded cubes outside the displaced area. (Figure $3.2 \mathrm{~g}-\mathrm{h}$ ) This occurs when the local temperature exceeds the thermal decomposition temperature of polystyrene. 

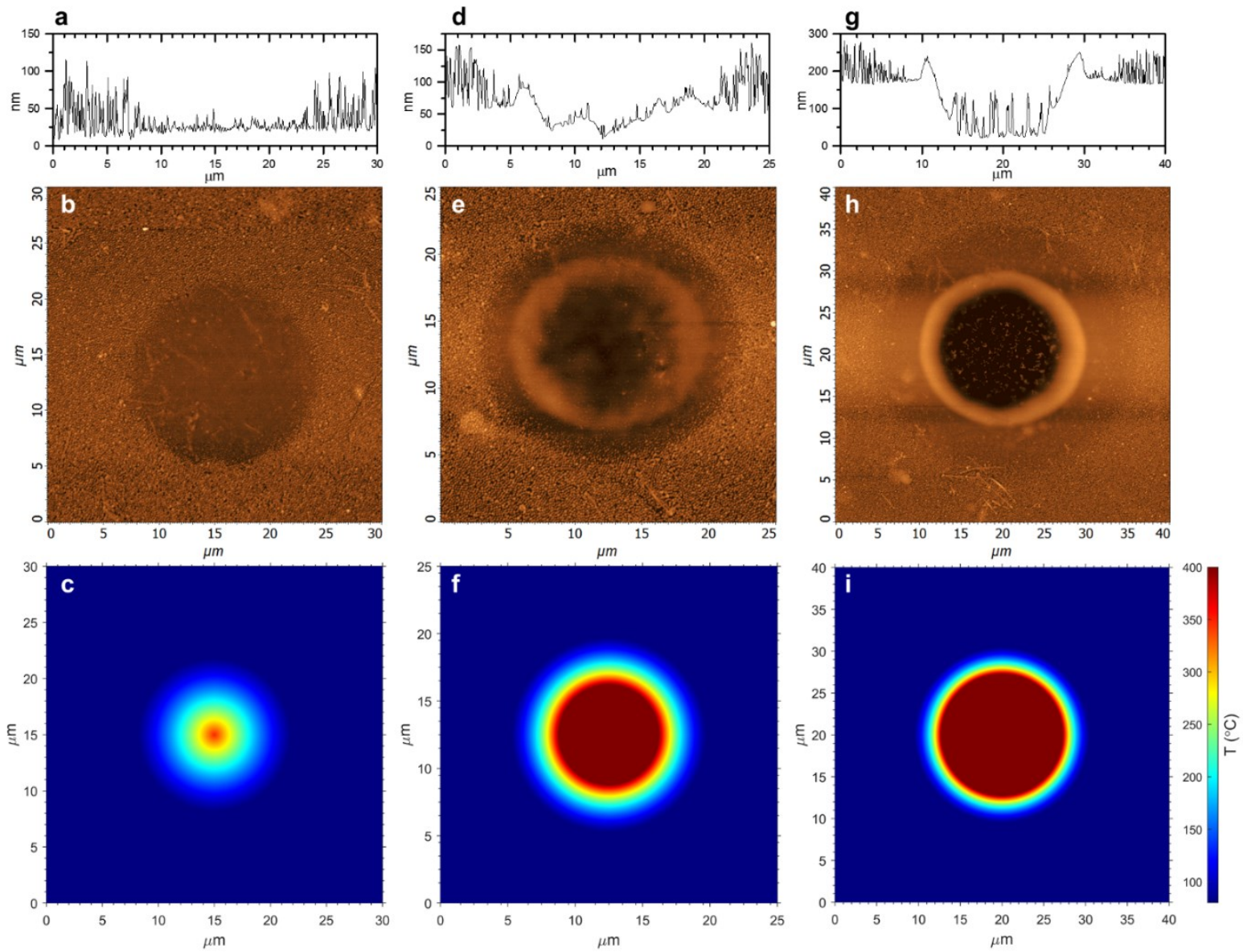

Figure 3.2. AFM images (b,e,h) of patterned areas in state I, II and III respectively, and associated cross sections made horizontally across the center of the image $(a, d, g)$ showing the topography. State I $(a, b, c)$ is defined by the observation of embedded nanocubes, with no deformation of the polymer layer (75 mW, 568 $\mathrm{nm}$ shown). In state II (d,e,f) the nanocrystals are highly embedded with a deformation of the surface polymer (20 mW, $476 \mathrm{~nm}$ shown), and in state III (g,h,i) ablation of the polymer is observed in the center (50 mW, 458 $\mathrm{nm}$ shown). A map of the calculated temperature (c,f,i) accompanies each AFM image, showing the patterned area to be the size of areas predicted to be heated to $T>T_{g}$.

\subsubsection{Temperatures obtained by photothermal heating}

The temperature of an isolated shaped particle $\left(\Delta \mathrm{T}_{\mathrm{np}}\right)$ can be calculated by: ${ }^{26}$

$$
\Delta T_{n p}=\frac{P_{a b s}}{4 \pi \beta r_{e q} \kappa}=\frac{\sigma_{a b s} I}{4 \pi \beta r_{e q} \kappa}
$$

where $P_{a b s}$ is the power absorbed by the nanoparticle, which can be defined by the irradiance of incident radiation $(I)$ and its absorption cross section $\left(\sigma_{a b s}\right)$ which was obtained as a function of wavelength from FDTD modelling. This is the most sensitive parameter of the temperature increase since this value varies largely with particle size; $\sigma_{a b s}$ was calculated as the average for the size distribution of the particles. The denominator describes the thermal capacitance of the nanoparticle, where $K$ is the thermal conductivity of the surroundings. For a nanoparticle at an interface it is 
convenient to define $K=1 / 2\left(\kappa_{1}+K_{2}\right)$, the average thermal conductivity of the media $\kappa_{1}$ and $\kappa_{2}$, which in this case are air and polystyrene ${ }^{27} \beta$ is a shape-correction coefficient and $r_{e q}$ is the radius of a sphere with an equivalent volume to the desired cube size. ${ }^{26}$ Due to the highly isotropic nature of the cubic shape it can be assumed $\beta \approx 1$.

Since the particles are homogeneously distributed throughout the illuminated area, the temperature of a particle at any given radius $(r)$ from the center of the beam is proportional to the irradiance from the Gaussian distribution of the focused beam:

$$
I(r)=\frac{2 P}{\pi \omega_{0}^{2}} e^{-2\left(\frac{r^{2}}{\omega_{0}^{2}}\right)}
$$

Where $P$ and $\omega_{0}$ is the waist radius of the incident beam. The radius was determined to be between 7.3 and $10.5 \mu \mathrm{m}$ depending on wavelength using the knife-edge technique. ${ }^{28}$ Through the combination of (3.1) and (3.2) we estimate the initial surface temperature of the nanocubes upon excitation. It should be noted the modelled temperatures only describe the initial temperature of the particles before any changes to the system. As the nanoparticles embed into the polymer $K_{e n v}$ is effectively altered, likewise accompanied with the alteration of their absorption spectra and hence $\sigma_{a b s .}$ Further changes in the polymer state such as its melting or decomposition would result in more alterations to these terms, making it difficult to predict the temperature throughout the entire embedment process.

When considering only the heating of isolated particles the predicted surface nanoparticle temperatures are considerably lower than the $T_{g}$ of the polystyrene thin film $\left(90-100{ }^{\circ} \mathrm{C}\right)$. For example, using the conditions presented in Figure $3.2 \mathrm{~d}$ surface temperatures of individual nanoparticles are only estimated to reach a maximum of $\sim 29{ }^{\circ} \mathrm{C}$ (Figure D.3). While the $\mathrm{T}_{\mathrm{g}}$ of polystyrene thin films can be depressed down to $75{ }^{\circ} \mathrm{C}$ in the case of ultra-thin films, ${ }^{29}$ this is insufficient to account for the discrepancy between the calculated and the predicted values due to equation (1) fails to account for the collective heating of the nanocubes. ${ }^{30}$

In an array of nanoparticles, collective heating can strongly contribute to the heating of the interparticle medium as the collective heating in a two dimensional system scales with the square 
of the interparticle distance. ${ }^{31}$ To quantify the temperature increase of the nanoparticle array $\left(\Delta T_{s}\right)$ we used the approach developed by Baffou et al. ${ }^{30}$ :

$$
\Delta T_{s}(r)=\int_{0}^{2 \pi} \int_{r}^{\infty} \frac{\sigma_{a b s} I(r) d r d \theta}{4 \pi \kappa A}
$$

where $A$ is the unit cell area, in this case approximated as $d^{2}$ where $d$ is the average interparticle distance. In this case we do not distinguish temperature contribution of individual nanoparticles and their external temperature as the heating is highly delocalized; the temperature of the nanoparticles is not significantly higher than that of the surrounding matrix. Baffou et al., define a confinement parameter $(\zeta)$ which predicts whether the particles are heated in a localized regime with the increased temperatures located around each particle, or a delocalized regime where a smooth temperature distribution is observed throughout the assembly of particles. ${ }^{30}$ The confinement parameter in this case is calculated to be $\zeta=0.015$ which predicts that $\Delta T_{n p}$ contributes only $1.5 \%$ to $\Delta T_{s}$.

The modelled temperatures including the contribution of collective heating are displayed in the bottom panel of Figure 3.2. The model shows agreement with the associated AFM images as the radius at which $\Delta T_{s}<90^{\circ} \mathrm{C}$ is consistent with the embedded area of the spot. The radius is slightly lower in the model as it does not consider conductive heat transfer over the area; it only models the collective heat of generated among adjacent unit cells.

\subsubsection{Experimental verification of Temperature}

To confirm the calculated temperatures, the temperature of the system was measured by Stokes/anti-Stokes Raman spectroscopy. The anti-Stokes signal arises from populated excited states in the probe molecule and is thus highly temperature sensitive. ${ }^{32}$ The ratio between the stokes and anti-stokes intensities can effectively used as a localized nanothermometer in surfaceenhanced Raman spectroscopy measurements: ${ }^{33}$

$$
\frac{I_{A S}}{I_{S}}=\left(\frac{\omega_{l}+\omega_{v}}{\omega_{l}-\omega_{v}}\right)^{4} \exp \left(\frac{\hbar \omega}{k T}\right)
$$

where $I_{A S}$ and $I_{S}$ are the stokes and anti-stokes Raman intensities, and $\omega_{\mathrm{I}}$ and $\omega_{\mathrm{v}}$ are laser and vibrational frequencies respectively. 
Stokes/anti-Stokes spectra were measured at $488 \mathrm{~nm}$ between 2- $50 \mathrm{~mW}$ on sample. Since the intensity of the Raman signal is also power-dependant, the system was initially calibrated by heating the sample up from $0-90{ }^{\circ} \mathrm{C}$. Figure 3.3 displays this temperature calibration, as well as the Stokes and anti-Stokes (aS/S) signals of the $773 \mathrm{~cm}^{-1}$ mode of Rh6G at $25^{\circ} \mathrm{C}$ and $90{ }^{\circ} \mathrm{C}$.

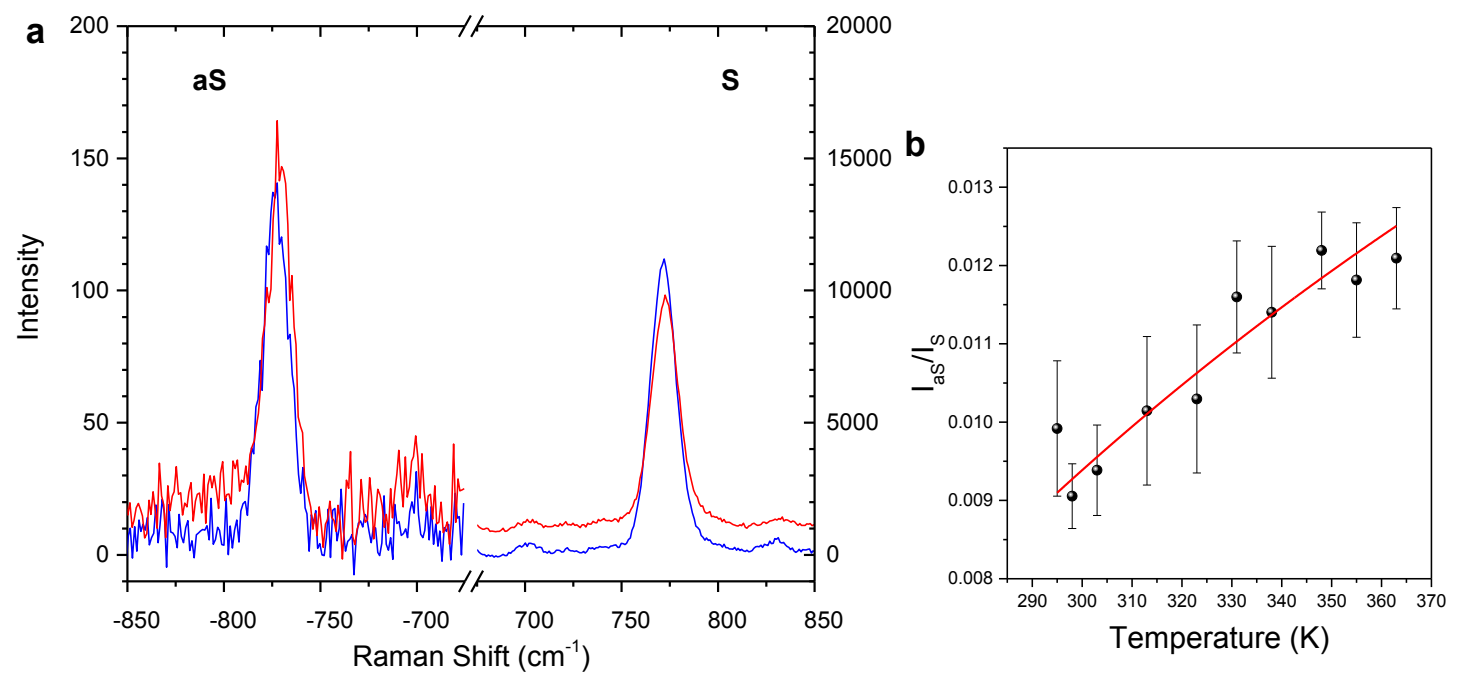

Figure 3.3 Stokes/anti-Stokes surface-enhanced Raman spectroscopy of Rh6G on AgNC monolayers a) the signal at $773 \mathrm{~cm}^{-1}$, note the scale of intensity is $10^{2}$ higher for the Stokes spectrum b) Temperature calibration of the aS/S measurements.

The temperature/intensity for SERS measurements were fitted (Figure 3.3 ) using:

$$
\frac{I_{A S}}{I_{S}}=A_{i}\left(\frac{\omega_{l}+\omega_{v}}{\omega_{l}-\omega_{v}}\right)^{4} \exp \left(\frac{\hbar \omega}{k T}\right)+C
$$

ere $A_{i}$ is the asymmetry factor representing the different SERS enhancement at the stokes and antistokes vibrational frequencies, and $C$ is a correction factor which was required to account for instrument response differences at these frequencies. The calibration was used to estimate the temperature with different incident powers (Figure 3.3). The temperature is measured up until the threshold power for embedment is observed. Since the embedment process significantly alters the LSPR signature of the AgNC's, the SERS enhancement would likely be affected, invalidating the calibration (Figure 3.3b). The temperature at the onset of embedment was $75 \pm 5{ }^{\circ} \mathrm{C}$, which was lower than expected but in the vicinity of the $T_{g}$ of a polystyrene thin film. ${ }^{29}$ This confirms the onset of the embedment process with the $T_{g}$ of polystyrene. It should be noted that SERS effects potentially introduce significant error in Stokes/anti-Stokes Raman thermometry due to the spatial inhomogeneity of the enhancement factor and experienced temperature of the probe molecules. ${ }^{34}$ 

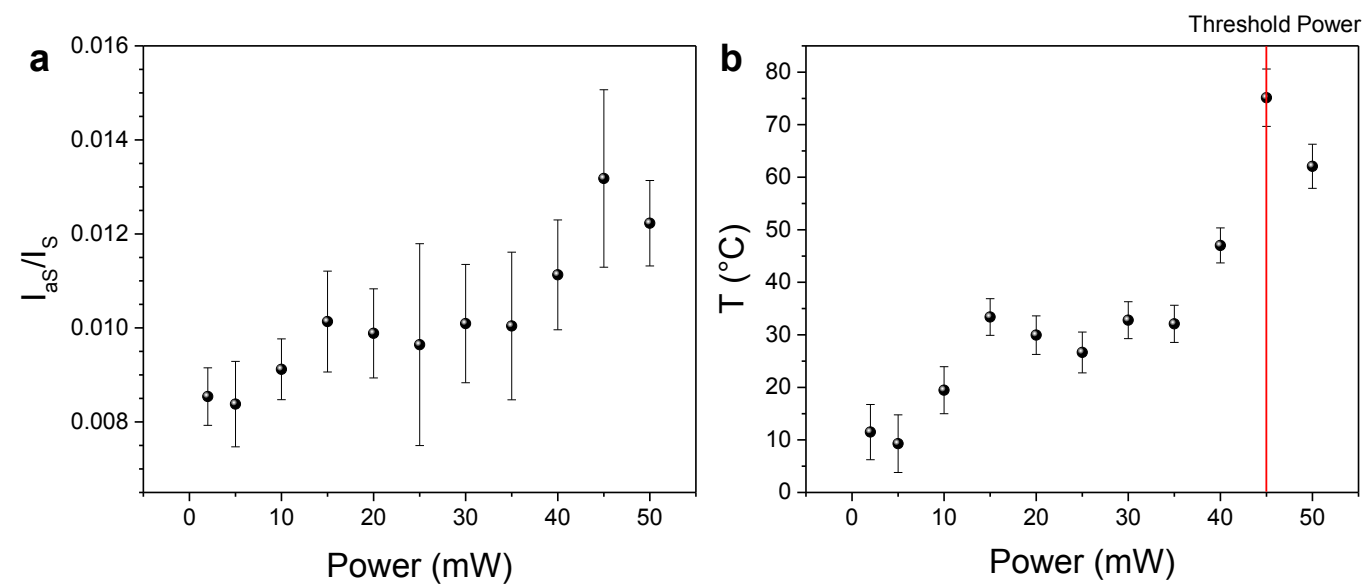

Figure 3.4 a) measured Stokes/anti-Stokes intensities as a function of power and b) determinations of temperature from them, with the threshold power at which embedment is observed is noted.

\subsubsection{Optical Patterning}

Of the three stages identified previously, state I is the most appealing for optical patterning due to the highly sensitive shift of the nanocubes' LSPR allowing for pronounced color changes. The observed topographical changes this state are consistent with heating past the $T_{g}$ of the polystyrene thin film $\left(90-100{ }^{\circ} \mathrm{C}\right) \cdot{ }^{35}$ At this temperature the nanoparticles will spontaneously incorporate themselves into the polymer film driven by a reduction in total surface energy. ${ }^{17} \mathrm{AFM}$ imaging determined that the particles embedded up to $39 \pm 5 \mathrm{~nm}$ into the polymer, or $70 \%$ of their edge length. In addition, optical properties corresponding to the state I transformation undergo significant alterations.

Figure 3.5 shows the transmittance and reflectance spectra of photothermally embedded nanoparticles contrasted $(a, c)$ against those embedded by conventional heating $(b, d)$. A $15 \mathrm{~nm}$ shift in the dipolar plasmon peak (D) is observed and a further shift of the quadrupolar plasmon mode (Q) significantly alters the profile of the transmission spectrum (Figure 3.5). A $45 \mathrm{~nm}$ shift and an increase in maximum reflectance by $15 \%$ in addition to a broad-band increase in reflectance produces a visible change in color and luster in the patterned area. These changes can be made with peak optical intensities as low as $0.087 \mathrm{~mW} / \mu \mathrm{m}^{2}$ at a wavelength closest to the LSPR resonance of the nanoparticles $(458 \mathrm{~nm})$. This state is also observed at longer wavelengths, albeit requiring more power where the absorption cross-section $\sigma_{\text {abs }}$ is minimal. 


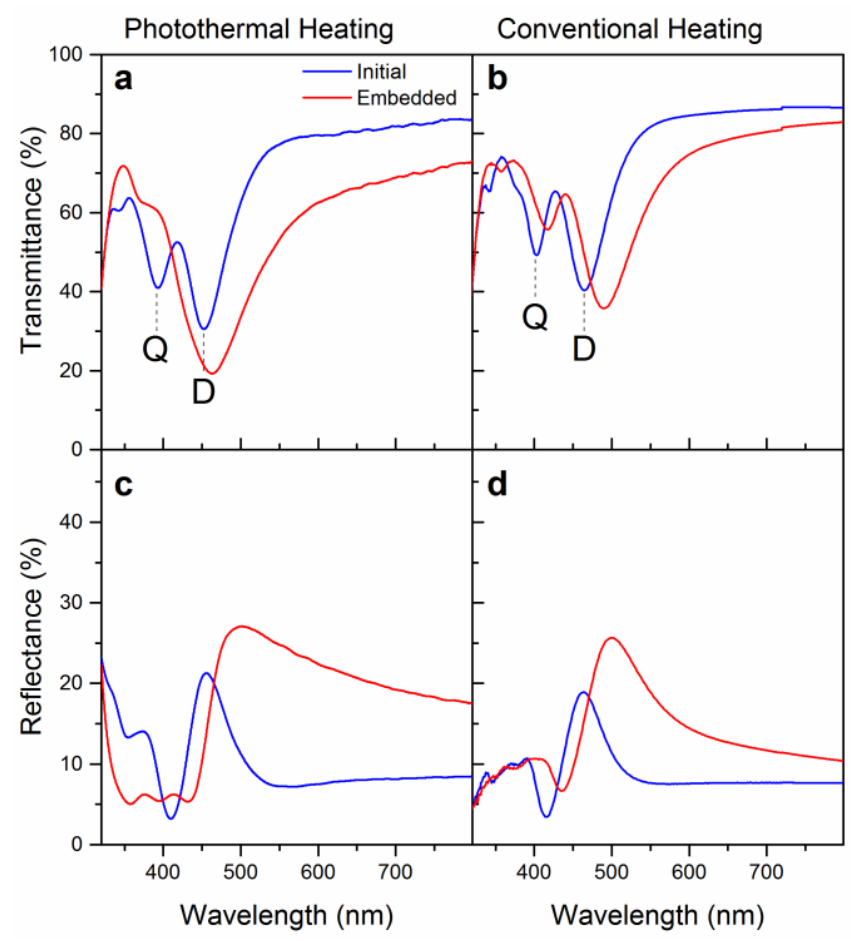

Figure 3.5. a,b) Transmittance spectra of a section of the film which before (blue) and after (red) being embedded photothermally (a), and using conventional heating (b). Transmittance spectra reveal a slight shift in the dipolar plasmon mode and a significant shift in the quadrupolar plasmon mode, more so in the case of photothermal embedment. Associated reflectance spectra, c,d) show a marked increase in reflectance upon embedment.

At this point the results only clearly distinguish two states: an initial state and fully embedded particles. However, it should be noted there exists a continuum of states where the particles are partially embedded and thus the technique could be expanded by utilizing partially embedded particles to create a greater variety of optical responses. The ability to generate or write a palette of plasmon-generated colors has garnered interest with direct applications in security, cryptography and information storage. ${ }^{36}$ Many strategies for plasmonic color generation rely on complex top-down fabrication techniques such as electron-beam lithography. ${ }^{11,37,38}$ However with this strategy using simple bottom-up fabrication new colors can be actively generated with simple continuous-wave laser exposure. The color palette of this technique can potentially be expanded. ${ }^{39}$

\subsubsection{Ablation of polystyrene films}

The second state observed upon the nanocomposite exposure to laser illumination is a transitionary state between states I and III. A deformation of the surface is observed which is the result of photomechanical stress caused by the thermal expansion of the polymer above the $T_{g}$ being bound by the more solid surrounding region. ${ }^{40}$ The predicted temperature is consistent onset 
temperature of the thermal decomposition of polystyrene $\left(>270{ }^{\circ} \mathrm{C}\right)$. In this case near-full embedment of the nanocubes can be observed; the majority of the particles are not discernable in the AFM topology (Figure 3.2e).

In the final and third state complete thermal decomposition of polystyrene in the film was observed, leaving a hole in the substrate. Furthermore, large amounts of the polymer have been displaced from the illuminated area by the convective transfer of heat forming a sizable ridge. Ablation depths vary depending on absorbed power and wavelength, but complete ablation ( 200 $\mathrm{nm}$ depth) was observed after an upper threshold was reached. The calculated temperatures (Figure 3.2i) of $>400{ }^{\circ} \mathrm{C}$ in the ablated areas are consistent with the expected temperatures required to ablate a hole of this depth in polystyrene. ${ }^{40}$ At these temperatures polystyrene undergoes thermal decomposition into volatile components allowing the material to be selectively removed from the film. ${ }^{41}$ Outside of the decomposed area a gradient in the embedded cubes' depth is observed. This area was found to lie outside the waist diameter of the beam where the light intensity and thus degree of photothermal heating is negligible; the embedment of nanocrystals is therefore the result of thermal dissipation from the central region.

\subsubsection{Control over optical patterning}

The resultant state of the polymer film after exposure was dependent on the power absorbed by the nanoparticles (Figure 3.6). Wavelengths which are on resonant with the LSPR of the nanoparticles patterned substrate with ease. Patterning at $458 \mathrm{~nm}$ was achieved with a peak intensity as low as $0.057 \mathrm{~mW} / \mu \mathrm{m}^{2}$ and complete ablation occurs at $0.434 \mathrm{~mW} / \mu \mathrm{m}^{2}$. At longer wavelengths where $\sigma_{a b s}$ is minimal, more power is required to drive the observable changes in polymer state. Obtaining embedded particles (State I) was easily achieved over a wide range of laser intensities while limiting the patterning to this state at resonant wavelengths required fine control. At $647 \mathrm{~nm} \sigma_{\mathrm{abs}}$ is so weak that the patterned area did not fit the profile of the laser beam, but instead produced sporadically embedded particles (Figure D.4). This displays how collective heating is dominant in this system, as the quasi-disordered particles only embed in localized areas of high particle density. 


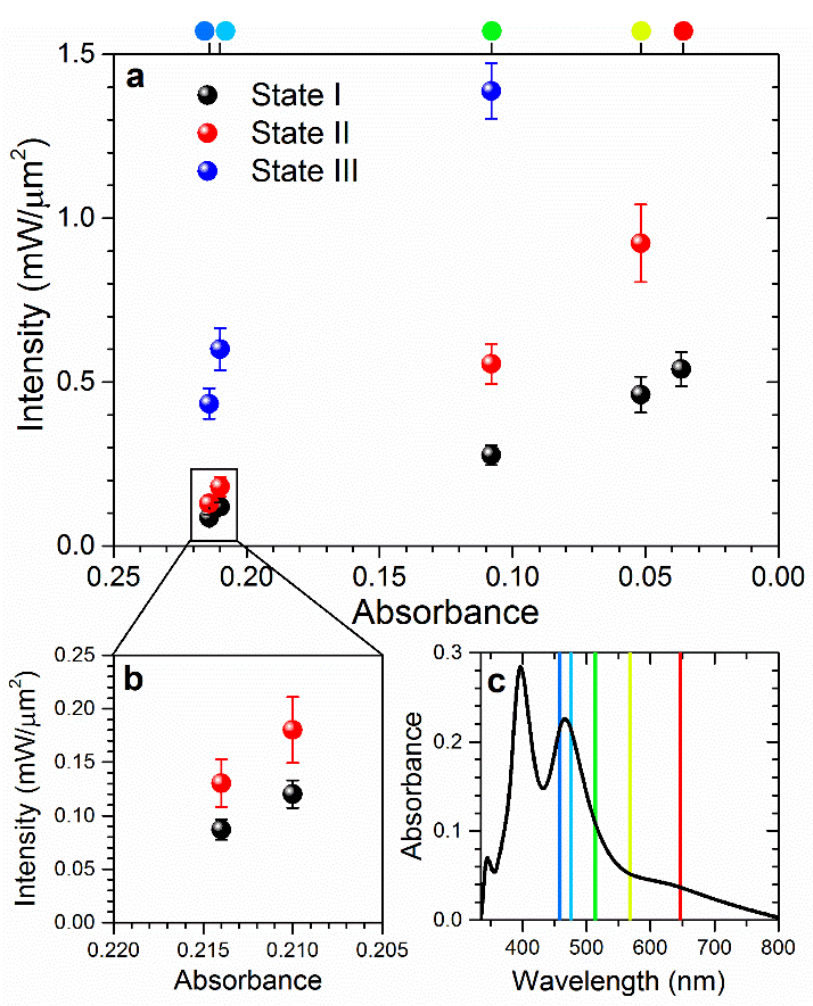

Figure 3.6. a,b) The threshold intensity as a function of absorbance of the silver nanocube films. The absorbance spectrum is shown in c) for reference. Weaker absorbance (at higher wavelengths) increase the threshold power required for patterning, though also slows for easier control over the final state.

Since the observed morphological changes occur on a rapid timescale the laser can be scanned across the surface to produce a desired pattern of any state. This can be done using a focused low-intensity, visible, CW light source; the equipment requirements are minimal. Other optical lithography techniques on transparent polymers typically use deep-UV or pulsed laser sources, or require the polymer to be loaded with a dye..$^{40}$ In the case of dye loaded polymers, the process permanently stains the polymer which could be undesirable. The nanoparticle coating can be removed after patterning with weak nitric acid leaving a transparent patterned film. This technique has also been demonstrated to be purely photothermal as well; no photocatalytic are non-linear optical processes are required to predict the results.

With this technique the precise, nanoscale control the position of the nanocubes in the axial direction is demonstrated, though the radial resolution is kept at the microscale. Plasmonic particles can also be used as localized point-sources of heat and the resolution of the technique can be improved. While in this work the heating is largely delocalized, thermoplasmonic heating can be 
localized to the immediate vicinity or even within the plasmonic nanostructure. ${ }^{42}$ This yields a potential route to sub-diffraction limit patterning by using a template of plasmonic nanostructures.

\subsection{Conclusion}

The use of silver nanocubes as point-heat sources for micro-scale optical patterning and lithography was demonstrated. We observed three distinct states defined by their own far-field optical properties and micro-scale topography. By taking of advantage of the unique spectral response of silver nanocubes a high degree of control over the patterning process can be obtained in comparison to higher order symmetric particles such spheres. ${ }^{13}$ This process was found to be purely photo-thermal; no other processes such as photo-catalysis or non-linear two-photon processes are required to explain the observed patterning. This work also highlights the role of collective heating as opposed to isolating heating and its impact on opto-lithographic applications. This process can be used to produce unique polymer-nanomaterial composites in which the nanomaterials can be embedded into the polymer in a well-controlled manner. Fine tuning of the process allows for control over the local environment of the nanocubes and thus control over the optical properties such as transmission, reflection and color and thus this technique can potentially be applied to plasmonic color generation. The ability for photothermal ablation of the polymer films allows this to be applied to the simple direct-write optical lithography of thin films or 2D materials. This work was performed in a highly delocalized regime allowing for consistent micro-scale patterning, but there exists a potential to make use of localized plasmon heating for expanding this technique towards performing sub-diffraction limit optical patterning and lithography. 


\subsection{References}

(1) Baffou, G.; Quidant, R. Thermo-Plasmonics: Using Metallic Nanostructures as NanoSources of Heat. Laser Photonics Rev. 2013, 7 (2), 171-187.

(2) Govorov, A. O.; Richardson, H. H. Generating Heat with Metal Nanoparticles. Nano Today 2007, 2 (1), 30-38.

(3) Baffou, G.; Rigneault, H. Femtosecond-Pulsed Optical Heating of Gold Nanoparticles. Phys. Rev. B - Condens. Matter Mater. Phys. 2011, 84 (3), 1-13.

(4) Huang, X. H.; El-Sayed, I. H.; Qian, W.; El-Sayed, M. a. Cancer Cell Imaging and Photothermal Therapy in the Near-Infrared Region by Using Gold Nanorods. J. Am. Chem. Soc. 2006, 128 (6), 2115-2120.

(5) Stern, J. M.; Stanfield, J.; Kabbani, W.; Hsieh, J. T.; Cadeddu, J. A. Selective Prostate Cancer Thermal Ablation with Laser Activated Gold Nanoshells. J. Urol. 2008, 179 (2), 748-753.

(6) Robert, H. M. L.; Kundrat, F.; Bermúdez-Ureña, E.; Rigneault, H.; Monneret, S.; Quidant, R.; Polleux, J.; Baffou, G. Light-Assisted Solvothermal Chemistry Using Plasmonic Nanoparticles. ACS Omega 2016, 1, 2-8.

(7) Boyd, D. A.; Greengard, L.; Brongersma, M.; El-Naggar, M. Y.; Goodwin, D. G. PlasmonAssisted Chemical Vapor Deposition. Nano Lett. 2006, 6 (11), 2592-2597.

(8) Neumann, O.; Urban, A. S.; Day, J.; Lal, S.; Nordlander, P.; Halas, N. J. Solar Vapor Generation Enabled by Nanoparticles. ACS Nano 2013, 7 (1), 42-49.

(9) Lee, B. J.; Park, K.; Walsh, T.; Xu, L. Radiative Heat Transfer Analysis in Plasmonic Nanofluids for Direct Solar Thermal Absorption. J. Sol. Energy Eng. 2012, 134 (2), 021009.

(10) Huang, W.; Qian, W.; El-Sayed, M. A. Photothermal Reshaping of Prismatic Au Nanoparticles in Periodic Monolayer Arrays by Femtosecond Laser Pulses. J. Appl. Phys. 2005, 98 (11).

(11) Zhu, X.; Vannahme, C.; Højlund-Nielsen, E.; Mortensen, N. A.; Kristensen, A. Plasmonic Colour Laser Printing. Nat. Nanotechnol. 2016, 11 (4), 325-329.

(12) Tokarev, I.; Minko, S. Tunable Plasmonic Nanostructures from Noble Metal Nanoparticles and Stimuli-Responsive Polymers. Soft Matter 2012, 8 (22), 5980-5987.

(13) Zillohu, A. U.; Abdelaziz, R.; Hedayati, M. K.; Emmler, T.; Homaeigohar, S.; Elbahri, M. Plasmon-Mediated Embedding of Nanoparticles in a Polymer Matrix: Nanocomposites Patterning, Writing, and Defect Healing. J. Phys. Chem. C 2012, 116 (32), 17204-17209.

(14) Lin, L.; Li, J.; Li, W.; Yogeesh, M. N.; Shi, J.; Peng, X.; Liu, Y.; Rajeeva, B. B.; Becker, M. F.; Liu, Y.; Akinwande, D.; Zheng, Y. Optothermoplasmonic Nanolithography for OnDemand Patterning of 2D Materials. Adv. Funct. Mater. 2018, 28 (41), 1803990.

(15) Pan, L.; Park, Y.; Xiong, Y.; Ulin-Avila, E.; Wang, Y.; Zeng, L.; Xiong, S.; Rho, J.; Sun, C.; Bogy, D. B.; Zhang, X. Maskless Plasmonic Lithography at $22 \mathrm{Nm}$ Resolution. Sci. Rep. 2011, 1, 1-6.

(16) Bushell, M.; lanoul, A. Selective Embedment of Silver Nanocrystals into Spatially Segregated Domains in Thin Polymer Films for Controlled Fabrication of Functional Nanocomposites. RSC Adv. 2019, 9 (33), 19131-19141.

(17) Bottomley, A.; Prezgot, D.; Coyle, J. P.; lanoul, A. Dynamics of Nanocubes Embedding into Polymer Films Investigated via Spatially Resolved Plasmon Modes. Nanoscale 2016, 
8 (21), 11168-11176.

(18) Zhang, S.; Bao, K.; Halas, N. J.; Xu, H.; Nordlander, P. Substrate-Induced Fano Resonances of a Plasmonic Nanocube: A Route to Increased-Sensitivity Localized Surface Plasmon Resonance Sensors Revealed. Nano Lett. 2011, 11 (4), 1657-1663.

(19) Lynch, D. W.; Hunter, W. R. Comments on the Optical Constants of Metals and an Introduction to the Data for Several Metals. In Handbook of Optical Constants of Solids; Elsevier, 1997; pp 275-367.

(20) König, T. A. F.; Ledin, P. A.; Kerszulis, J.; Mahmoud, M. A.; El-Sayed, M. A.; Reynolds, J. R.; Tsukruk, V. V. Electrically Tunable Plasmonic Behavior of Nanocube-Polymer Nanomaterials Induced by a Redox-Active Electrochromic Polymer. ACS Nano 2014, 8 (6), 6182-6192.

(21) Sultanova, N.; Kasarova, S.; Nikolov, I. Dispersion Properties of Optical Polymers. Acta Phys. Pol. A 2009, 116 (4), 585-587.

(22) Su, K. H.; Wei, Q. H.; Zhang, X.; Mock, J. J.; Smith, D. R.; Schultz, S. Interparticle Coupling Effects on Plasmon Resonances of Nanogold Particles. Nano Lett. 2003, 3 (8), 1086-1090.

(23) Bouhelier, A.; Bachelot, R.; Im, J. S.; Wiederrecht, G. P.; Lerondel, G.; Kostcheev, S.; Royer, P. Electromagnetic Interactions in Plasmonic Nanoparticle Arrays. J. Phys. Chem. B 2005, 109 (8), 3195-3198.

(24) Zhao, L.; Kelly, K. L.; Schatz, G. C. The Extinction Spectra of Silver Nanoparticle Arrays: Influence of Array Structure on Plasmon Resonance Wavelength and Width. J. Phys. Chem. B 2003, 107 (30), 7343-7350.

(25) Bushell, M.; Bottomley, A.; lanoul, A. Utilization of Hybrid Plasmonic Modes to Investigate Surface Interactions between Nanocubes and Polymer Substrates. Appl. Phys. A Mater. Sci. Process. 2017, 123 (2), 1-7.

(26) Baffou, G.; Quidant, R.; García De Abajo, F. J. Nanoscale Control of Optical Heating in Complex Plasmonic Systems. ACS Nano 2010, 4 (2), 709-716.

(27) Baffou, G.; Quidant, R.; Girard, C. Thermoplasmonics Modeling: A Green's Function Approach. Phys. Rev. B - Condens. Matter Mater. Phys. 2010, 82 (16), 1-11.

(28) Suzaki, Y.; Tachibana, A. Measurement of the Mm Sized Radius of Gaussian Laser Beam Using the Scanning Knife-Edge. Appl. Opt. 1975, 14 (12), 2809.

(29) Vignaud, G.; Chebil, M. S.; Bal, J. K.; Delorme, N.; Beuvier, T.; Grohens, Y.; Gibaud, A. Densification and Depression in Glass Transition Temperature in Polystyrene Thin Films. Langmuir 2014, 30 (39), 11599-11608.

(30) Baffou, G.; Berto, P.; Berm, E.; Quidant, R.; Monneret, S.; Bermúdez Ureña, E.; Quidant, R.; Monneret, S.; Polleux, J.; Rigneault, H. Photoinduced Heating of Nanoparticle Arrays. ACS Nano 2013, 7 (8), 6478-6488.

(31) Govorov, A. O.; Zhang, W.; Skeini, T.; Richardson, H.; Lee, J.; Kotov, N. A. Gold Nanoparticle Ensembles as Heaters and Actuators: Melting and Collective Plasmon Resonances. Nanoscale Res. Lett. 2006, 1 (1), 84-90.

(32) Malyj, M.; Griffiths, J. E. Stokes/Anti-Stokes Raman Vibrational Temperatures: Reference Materials, Standard Lamps, and Spectrophotometric Calibrations. Appl. Spectrosc. 1983, 37 (4), 315-333.

(33) Maher, R. C.; Cohen, L. F.; Gallop, J. C.; Le Ru, E. C.; Etchegoin, P. G. TemperatureDependent Anti-Stokes/Stokes Ratios under Surface-Enhanced Raman Scattering Conditions. J. Phys. Chem. B 2006, 110 (13), 6797-6803.

Pozzi, E. A.; Zrimsek, A. B.; Lethiec, C. M.; Schatz, G. C.; Hersam, M. C.; Van Duyne, R. 
P. Evaluating Single-Molecule Stokes and Anti-Stokes SERS for Nanoscale Thermometry. J. Phys. Chem. C 2015, 119 (36), 21116-21124.

(35) Rieger, J. The Glass Transition Temperature of Polystyrene. J. Therm. Anal. 1996, 46 (34), 965-972.

(36) Kristensen, A.; Yang, J. K. W.; Bozhevolnyi, S. I.; Link, S.; Nordlander, P.; Halas, N. J.; Mortensen, N. A. Plasmonic Colour Generation. Nat. Rev. Mater. 2016, 2 (1), 16088.

(37) Tan, S. J.; Zhang, L.; Zhu, D.; Goh, X. M.; Wang, Y. M.; Kumar, K.; Qiu, C.; Yang, J. K. W. Plasmonic Color Palettes for Photorealistic Printing with Aluminum Nanostructures. Nano Lett. 2014, 14 (7), 4023-4029.

(38) Miyata, M.; Hatada, H.; Takahara, J. Full-Color Subwavelength Printing with GapPlasmonic Optical Antennas. Nano Lett. 2016, 16 (5), 3166-3172.

(39) Stewart, J. W.; Akselrod, G. M.; Smith, D. R.; Mikkelsen, M. H. Toward Multispectral Imaging with Colloidal Metasurface Pixels. Adv. Mater. 2017, 29 (6).

(40) Kappes, R. S.; Schönfeld, F.; Li, C.; Golriz, A. A.; Nagel, M.; Lippert, T.; Butt, H.-J. J.; Gutmann, J. S. A Study of Photothermal Laser Ablation of Various Polymers on Microsecond Time Scales. Springerplus 2014, 3 (1), 489.

(41) Tsunekawa, M.; Nishio, S.; Sato, H. Laser Ablation of Polymethylmethacrylate and Polystyrene at $308 \mathrm{Nm}$ : Demonstration of Thermal and Photothermal Mechanisms by a Time-of-Flight Mass Spectroscopic Study. J. Appl. Phys. 1994, 76 (9), 5598-5600.

(42) Magnozzi, M.; Proietti Zaccaria, R.; Catone, D.; O’Keeffe, P.; Paladini, A.; Toschi, F.; Alabastri, A.; Canepa, M.; Bisio, F. Interband Transitions Are More Efficient Than Plasmonic Excitation in the Ultrafast Melting of Electromagnetically Coupled Au Nanoparticles. J. Phys. Chem. C 2019, 123 (27), 16943-16950. 


\section{Chapter 4: Plasmonic Colour Generation in Silver Nanocrystal-over-Mirror Films by Thermal Embedment into a Polymer Spacer}

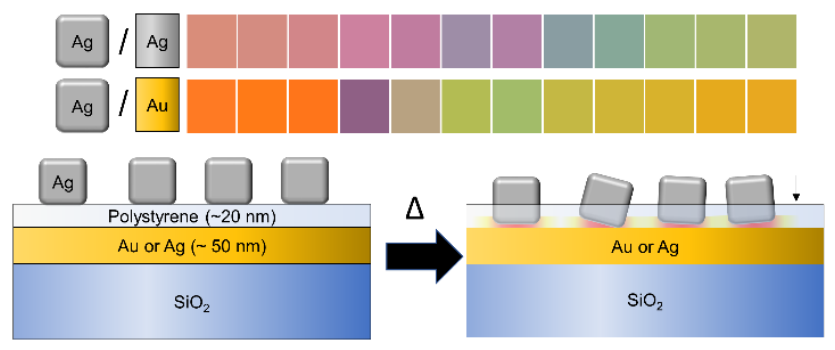

\section{Abstract}

The production of colours by plasmonic nanostructures is an attractive prospect over dyes as they allow ultra-high resolution, non-fading colours. Typical techniques for producing plasmonic colour patterns such as by electron beam or ion beam lithography are expensive, slow and not well scalable. This work demonstrates a simple, lithography-free technique for producing plasmonic colours using a silver nanocube (AgNC) based nanoparticle-over-mirror (NPoM) system with thermally-generated colours. AgNC's are deposited over a metal (Au or Ag) film with a polystyrene (PS) dielectric spacer. Upon heating of the system past the glass-transition temperature $\left(T_{g}\right)$ of PS, the AgNC embed into the polymer, reducing the AgNC / metal film distance. This results in a strong gap-plasmon which shifts over $200 \mathrm{~nm}$ across the visible spectrum. The system is demonstrated to be tunable across the visible range, producing wide, but distinct colour palettes depending on the metal film used. The technique can potentially be applied to plasmonic colour-patterning systems to produce high-resolution microscale or nanoscale patterns over a large area.

Prezgot, D.; Tatarchuk, S.W.; lanoul, A. Plasmonic Colour Generation in Silver Nanocrystal-overMirror Films by Thermal Embedment into a Polymer Spacer, Manuscript in Preparation 


\subsection{Introduction}

Sub-wavelength scale metal nanostructures exhibit unique light-matter interactions arising from the collective oscillation of free electrons. This phenomenon, the localized surface plasmon resonance (LSPR), is responsible for the scattering and absorbance of light, and unique near-field properties of plasmonic nanomaterials. The properties of the surface plasmon resonance are highly dependent on the composition, shape, size and local environment of the nanostructure. ${ }^{1-3}$ The most ancient and perhaps simplest application of plasmonic materials is to produce colours. ${ }^{4,5}$ However, the engineering of structurally based colours, particularly by manipulating the sensitive nature of the LSPR in plasmonic building blocks, is in fact an emerging field of research which has seen a surge in recent interest. ${ }^{6}$ Structural colours provide an alternative to dye-based pigments with potential for greater longevity, environmental sustainability and extreme resolution. Plasmonic colours have been printed down to 100000 dots per inch (dpi); colour patterning can be performed at a resolution below the diffraction limit. ${ }^{7-9}$ In addition to high resolution color patterning, ${ }^{10}$ plasmon-derived structural colours can applied to devices such as display technologies, ${ }^{11-13}$ anticounterfeiting devices, ${ }^{14}$ information storage, ${ }^{15}$ holography, ${ }^{16}$ and multispectral imaging filters. ${ }^{17,18}$

Typical methods used to produce plasmonic colours include electron beam lithography, ${ }^{19,20}$ ion beam lithography, ${ }^{10,21}$ and nano-imprint lithography. ${ }^{7}$ However, such lithographic techniques are expensive, time consuming and are not easily scalable to produce large-scale colour prints. Few examples exist which avoid making use of these techniques. ${ }^{13,22-25}$ It is thus desirable to find facile, low cost, scalable means of producing plasmonic-generated colours.

A common strategy to achieve wide gamut plasmon-derived colours is to make use of gap surface-plasmons. ${ }^{7-9,25,26}$ Metal-insulator-metal geometries structures support highly absorptive cavity plasmon resonances which are highly tunable based on the dielectric properties or metalmetal distance. ${ }^{27}$ These structures typically consist of a metal (Ag, Au or Al) particle or disc separated from a metal film by a dielectric spacer. A promising, lithography free, scalable architecture uses plasmonic particles deposited over a metal/dielectric composite film, often referred to as a "nanoparticle on mirror" (NPoM) geometry. One such system with a strongly absorptive and highly tunable resonances across the visible (and NIR) spectrum uses Ag 
nanocubes $(\mathrm{AgNC})$ over a Au film. ${ }^{18,28} \mathrm{~A}$ facile, single step method to locally control the particlefilm gap in NPoM systems remains challenging.

This work makes use of a previously demonstrated thermal embedment process we developed to finely control the interparticle gap between a AgNC layer and metal ( $\mathrm{Ag}$ or $\mathrm{Au}$ ) film with polystyrene as the dielectric spacer..$^{29,30} \mathrm{~A}$ controlled layer of well-spaced AgNCs are deposited on top of a thin $(\sim 20 \mathrm{~nm})$ polystryrene film over the metal thin film $(\sim 50 \mathrm{~nm})$. By heating the film past the glass transition temperature $\left(\mathrm{T}_{\mathrm{g}}\right)$ of polystyrene, the particle embeds into the polymer thereby decreasing the particle-mirror gap distance and red-shifting the cavity plasmon resonance. The process can be monitored in real-time, allowing for extrapolation of the achievable colour gamut, and the embedment can be stopped at any point to obtain a desired colour. Using $\mathrm{Ag}$ or $\mathrm{Au}$ as the metal film each display a distinct range of obtainable colours. Demonstrated is a facile, lithography free strategy for plasmon colour generation which has the potential to be scaled down to potentially to high resolution, sub-diffraction limit plasmonic colour printing.

\subsection{Experimental}

Materials: Silver nitrate (AgNO3, 99+\%), polyvinylpyrrolidone (PVP, MW 55,000), anhydrous ethylene glycol (EG, 99.8\%), sodium sulfide $\left(\mathrm{Na}_{2} \mathrm{~S}\right)$, and sodium chloride $(\mathrm{NaCl})$ were purchased from Sigma-Aldrich and used as obtained. Polystyrene beads (PS, MW 250 000) were obtained from Acros Organics. Trimethylaluminum ( $>98 \%$, TMA) was purchased from Strem and used as received.

Synthesis of silver nanocubes (AgNC). AgNCs were synthesized using a two-step chloridemediated polyol method. ${ }^{29}$ A round bottom flask containing $15 \mathrm{~mL}$ of ethylene glycol (EG) anhydrous $\sim 0.4 \mathrm{~g}$ of polyvinylpyrrolidone (PVP) $(\sim 55,000 \mathrm{MW})$ was allowed in a $150{ }^{\circ} \mathrm{C}$ oil bath heated for one hour under magnetic stirring. To the round bottom flask $200 \mu \mathrm{L}$ of $3 \mathrm{mM} \mathrm{Na} 2 \mathrm{~S}$ and $25 \mu \mathrm{L}$ of $0.45 \mathrm{M} \mathrm{NaCl}$ was added and allowed to stir for 1 minute, followed by $1.5 \mathrm{~mL}$ of $282 \mathrm{mM}$ $\mathrm{AgNO}_{3}$ at a rate of $0.5 \mathrm{~mL} / \mathrm{min}$ via a syringe pump. Aliquots of the reaction were monitored by UVVis spectroscopy. After $\sim 1 \mathrm{~h}$ when the reaction showed a plasmonic signature indicative of small AgNC with peak positions $\sim 405 \mathrm{~nm}$ and $\sim 355 \mathrm{~nm}$ for the dipolar and multipolar modes 
respectively, ${ }^{31}$ a further $1.5 \mathrm{~mL}$ of $\mathrm{AgNO}_{3}$ was injected. The reaction was monitored until the desired spectral signature was observed and then quenched in an ice bath. The reaction mixture was diluted 1:1 with EtOH and centrifuged at $12000 \mathrm{RCF}$ for 30 minutes, re-dispersed in $\mathrm{EtOH}$, and subsequently centrifuged twice more for 10 minutes.

Fabrication of Au/Ag thin films. $50 \mathrm{~nm}$ of Au or Ag films were deposited onto glass slides pe-cleaned in piranha solution (1:5 $\left.\mathrm{H}_{2} \mathrm{O}_{2}: \mathrm{H}_{2} \mathrm{SO}_{4}\right)$ by thermal evaporation on a Balzers BA510 system. $\mathrm{Au}$ deposition was preceded by deposition of a $10 \mathrm{~nm} \mathrm{Cr}$ adhesion layer.

$2 \mathrm{~nm}$ of $\mathrm{Al}_{2} \mathrm{O}_{3}$ was deposited over $\mathrm{Ag}$ films by atomic layer deposition (ALD). Depositions were performed using a Picosun R200 Advanced deposition tool. TMA and distilled water were both held at $18{ }^{\circ} \mathrm{C}$ in stainless steel bubblers for all depositions. The deposition recipe began with ten $0.2 \mathrm{~s}$ pulses of TMA, each separated by $10 \mathrm{~s}$ purges, to ensure initial surface saturation of TMA. This was immediately followed with 20 cycles of TMA and water pairs: a $0.2 \mathrm{~s}$ pulse of TMA with a $20 \mathrm{~s}$ purge and a $0.5 \mathrm{~s}$ pulse of water with a $60 \mathrm{~s}$ purge. The chamber was held at the deposition temperature of $120^{\circ} \mathrm{C}$ for 30 min prior to initiation of deposition to ensure uniformity.

Spin-coating of polystyrene (PS) thin films. PS solutions of $0.5 \mathrm{wt} \%$ in toluene were prepared, filtered through $0.22 \mu \mathrm{m}$ PTFE filters pre-rinsed with toluene and using glass syringes to avoid contamination. ${ }^{32}$ Films were using a Laurel Technologies WS-400-6NPP-LITE spin coater at 4000 rpm on Au films and $3000 \mathrm{rpm}$ on Ag films in order to produce $\sim 20 \mathrm{~nm}$ PS films. ${ }^{33}$ Films were allowed to dry for $2 \mathrm{~h}$ then annealed at $125^{\circ} \mathrm{C}$ for $16 \mathrm{~h}$.

Langmuir-Schaeffer Deposition. Silver nanocube monolayers were deposited onto the polystyrene films using the Langmuir-Schaeffer technique using a NIMA 311D Langmuir trough filled with milli Q water (1.8 M $\Omega$ ). AgNCs were dispersed in $300 \mu \mathrm{L}$ of chloroform and deposited onto the water surface using a micro-syringe. Monolayers were transferred onto PS thin films in the gas phase of the isotherm at a surface pressure of $0.5 \mathrm{mN} / \mathrm{m}$.

Thermal embedment. Samples placed on an aluminium heating block (Ocean Optics CUV-AL-UV) attached to a heat pump and heated to between $100-120^{\circ} \mathrm{C}$. UV-Vis reflectance spectra were monitored using fibre-optic spectrometer with a xenon lamp and reflectance probe (Ocean Optics 
USB2000, PX-2, R200-7-SR). Reflectance measurements were baselined to a silver reference mirror. Reflectance spectra were converted to CIE XYZ and SRGB values (See section 1.5.3).

Atomic Force Microscopy (AFM). The topographic measurements of the substrates were obtained using an Ntegra (NT-MDT) atomic force microscope in semi-contact mode using gold-reflexed cantilevers with rotated monolithic silicon tips (135 $\mu \mathrm{m}$ long, 0.3-6 N/m spring constant, resonance

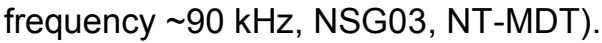

Numerical Modelling Finite-difference time-domain (FDTD) calculations were performed using a commercial solver (Lumerical FDTD Solutions, v8.16). The models consisted of a $60 \mathrm{~nm}$ silver nanocube with $12 \mathrm{~nm}$ corner radius and a $2 \mathrm{~nm}$ capping layer of PVP, with a thin polystyrene film over a metal film. Materials were described by an analytical model fitted to tabulated permittivity data. ${ }^{34-37} \mathrm{~A}$ plane-wave source was used with periodic $\mathrm{x}$ and $\mathrm{y}$ boundaries and perfectly matched layer z boundaries.

\subsection{Results and Discussion}

\subsubsection{Design of thermally-tunable NPoM composites}

Plasmon-derived colours were generated by tuning the particle-mirror distance in a $\mathrm{Ag}$ nanocube $(\mathrm{AgNC})$ over Au thin film NPoM system. The geometry of the system is presented in Figure 4.1a. The AgNC/Au system was chosen as it has been previously demonstrated to support a highly absorptive gap-plasmon resonances which are tunable across the visible spectrum and into the near-IR. ${ }^{17,28}$ Using a thin $\mathrm{Ag}$ film on the order of the size of the particle yields higher energy, narrow resonances that are the result of plasmon-hybridization both with the film's surface plasmons and the nanoparticle's image charge ${ }^{38}$ Polystyrene is used as a dielectric spacer where the thermal embedment process is used to tune the gap-mode frequency and control the plasmonderived colours. ${ }^{29}$ 


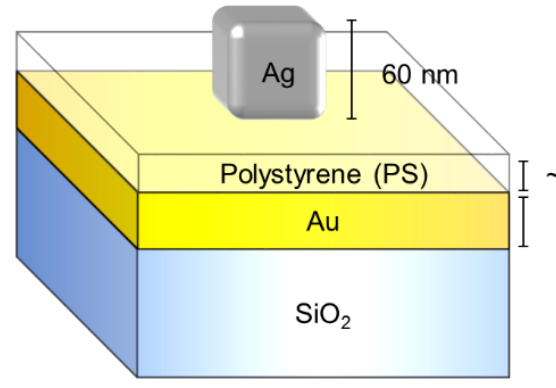

b

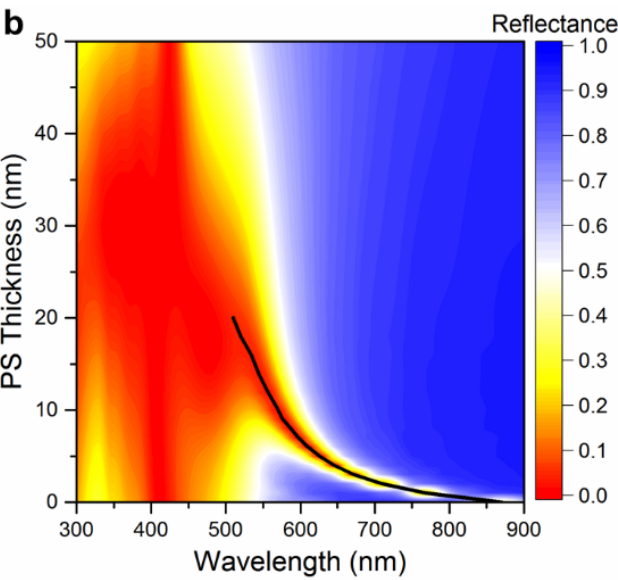

C

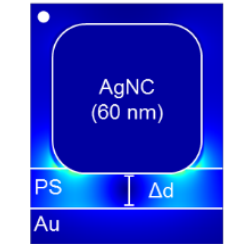

$50 \mathrm{~nm}$

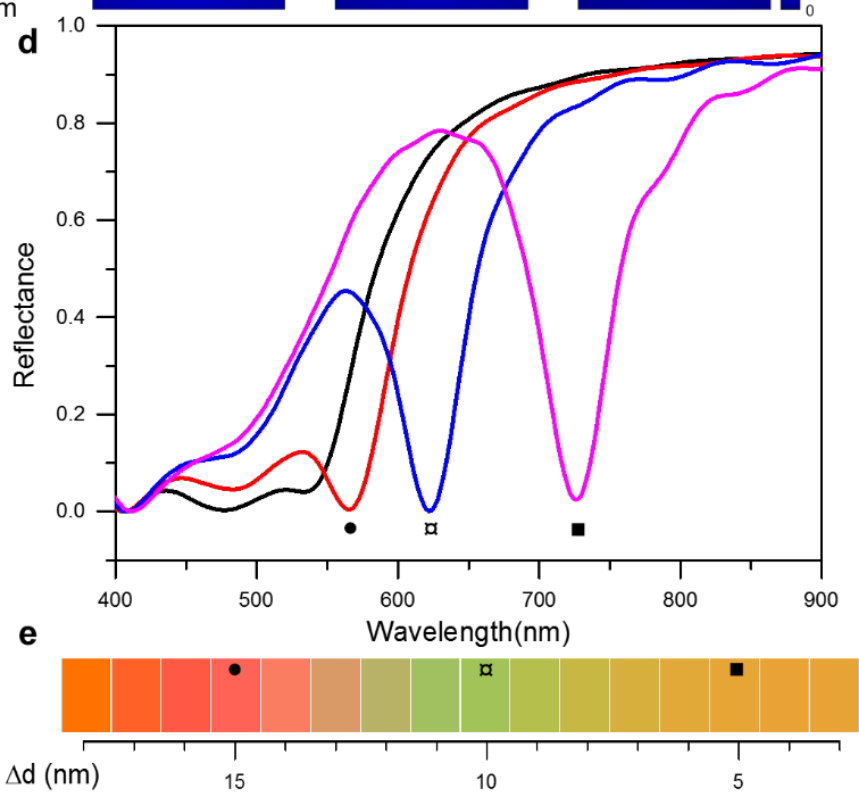

Figure 4.1. a) Schematic of the NPoM system, with a $60 \mathrm{~nm}$ AgNC over an Au film with polystyrene acting as the dielectric spacer. b) FDTD model showing the system with a polystyrene thickness from $0-50 \mathrm{~nm}$. Below $20 \mathrm{~nm}$ the gap plasmon becomes distinct and rapidly redshifts. c,d) FDTD model with a $16 \mathrm{~nm}$ polystyrene spacer as the nanocube is embedded into the polystyrene. Electric field enhancement $\left(\left|E_{\mid} / E_{0}\right|^{2}\right)$ at a gap distance of 15,10 and $5 \mathrm{~nm}$ and the associated calculated reflectance is shown. e) The predicted colour gamut of the system from converting calculated spectra to sRGB values.

The ideal thickness of polystyrene spacer was predicted using finite-difference timedomain (FDTD) modelling. The thermal embedment process has an upper limit to the extent which the silver nanocubes embed; the nanocubes can only be embedded up to $\sim 70 \%$ their edge length. Furthermore when embedding past $50 \%$ their edge length the nanocubes tilt, ${ }^{29}$ which would alter the efficiency of the gap plasmon. ${ }^{28}$ Ideally a film needed to be produced that was $<50 \%$ the edge length of the nanocube and also an appropriate distance to display a highly sensitive shift of the LSPR upon thermal embedment. Figure $4.1 \mathrm{~b}$ displays the results of FDTD calculations of a $60 \mathrm{~nm}$ Ag nanocube over a $50 \mathrm{~nm}$ Au film with polystyrene thicknesses varied between $0-50 \mathrm{~nm}$. The gap plasmon only becomes distinct from the AgNC LSPR and Au interband transitions at a distance $<20 \mathrm{~nm}$, and then shifts rapidly showing an increasing sensitivity as the AgNC/Au gap narrows. With an $18 \mathrm{~nm}$ polystyrene film the gap plasmon shifts from $534 \mathrm{~nm}$ to beyond the visible range (> 
$700 \mathrm{~nm}$ ) at distances $<5 \mathrm{~nm}$ (Figure 4.1d), resulting in little predicted colour change beyond this point (Figure 4.1e).

\subsubsection{Fabrication of AgNC/Au NPoM composites}

Fabrication of the AgNC over Au NPoM films was performed by the following steps (See experimental for details): i) deposition of $\sim 50 \mathrm{~nm}$ Au films onto standard glass microscope slides with $\mathrm{Cr}(\sim 10 \mathrm{~nm})$ adhesion layer by thermal evaporation, ii) spin coating of polystyrene films over the gold films, iii) deposition of AgNC's by the Langmuir - Schaeffer technique (Figure 4.2a).

Silver nanocubes were synthesized by the polyol method, ${ }^{29}$ producing nanocubes with an edge length of $60 \pm 14 \mathrm{~nm}$. Particles of this edge length were chosen as they display a strong substrate-induced hybridization, ${ }^{39}$ having strong, well separated peaks with little broadening. While the prepared colloid was somewhat polydisperse, its UV-Vis extinction profile was narrow, and displayed strong hybridization when deposited on polystyrene (Figure E.1). The deposited polystyrene films were found an actual thickness of $17 \pm 2 \mathrm{~nm}$ by AFM and were smooth, with an RMS roughness of $0.2 \mathrm{~nm}$. The Langmuir-Schaeffer technique allows for the deposition of the nanocubes on polystyrene in a well-controlled manner; a movable barrier can be used to control the particle density of the Langmuir film. This technique enables large scale transfer of nanoparticle assemblies in a reproducible manner. ${ }^{40}$

\subsubsection{Plasmonic colour generation in AgNC / Au NPoM films}

Plasmonic colours were controlled by thermal embedment of the silver nanocubes into the polystyrene substrate. Thermal embedment of the nanocubes was performed by heating the NPoM assembly to $>120^{\circ} \mathrm{C}$ and monitoring the process by reflection spectroscopy. After the sample exceeds the $T_{g}$ of polystyrene the nanocubes become embedded into the polymer. Since the polymer spacer is thin, a gap-plasmon becomes discernable above $\sim 550 \mathrm{~nm}$ and red-shifts up to $695 \mathrm{~nm}$ (Figure 4.2b). The embedment of the AgNCs was confirmed by atomic force microscopy (AFM) demonstrating the average heights of the nanocrystals had decreased by $21 \pm 4 \mathrm{~nm}$ (Figure 4.2c,d) which is on the same order of size as the polymer film itself suggesting maximal embedment of the nanoparticles. The results differ from theoretical predictions (Figure 4.1c) in that the minimum reflectance increases (or maximum absorptance decreases, Figure E.2), as well as becomes 
broader as the particles become further embedded into the PS. The system also displays a lower plasmon shift than predicted by theory, where full embedment results in a resonance $>900 \mathrm{~nm}$ ). These effects are likely due to the nature of the ensemble measurement; the experimental measurement has a degree of polydispersity in size, depth of immersion and orientation of the nanocubes all of which serve to broaden the resonance compared to the single particle model. ${ }^{28,29}$

a

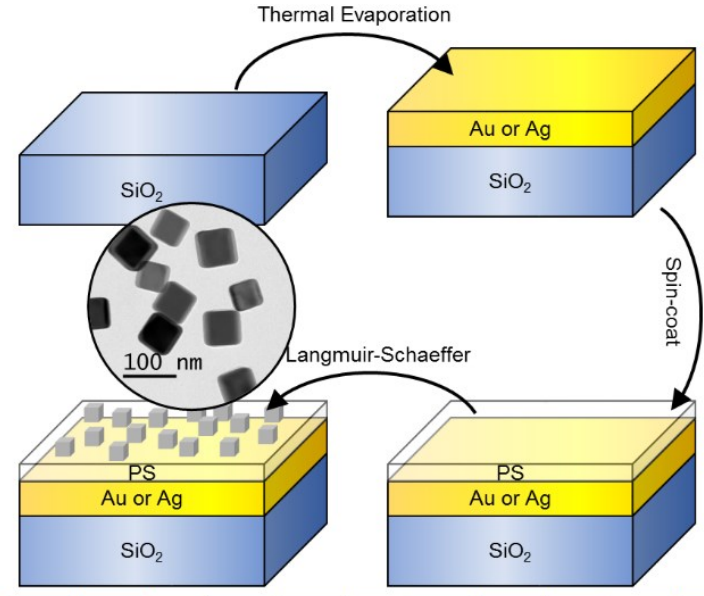

b

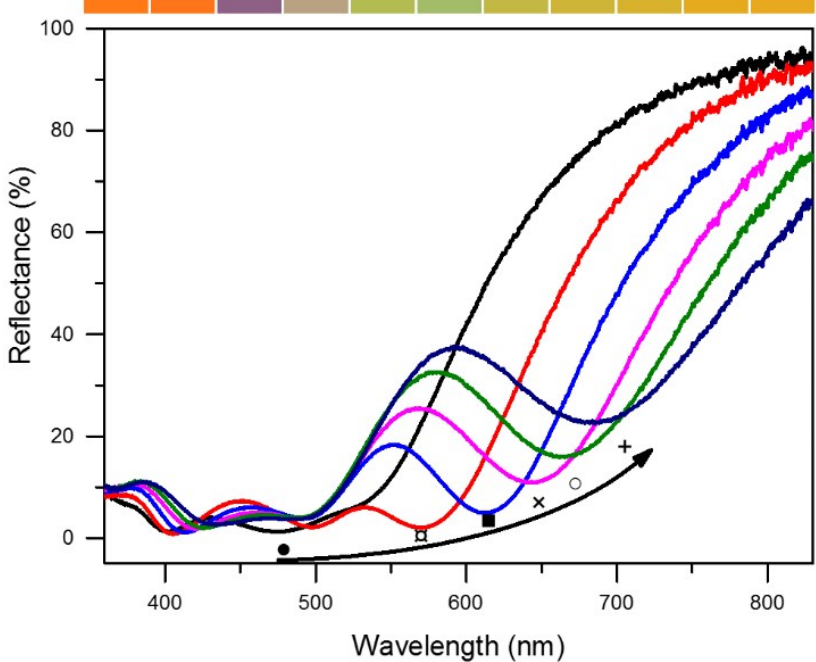

C
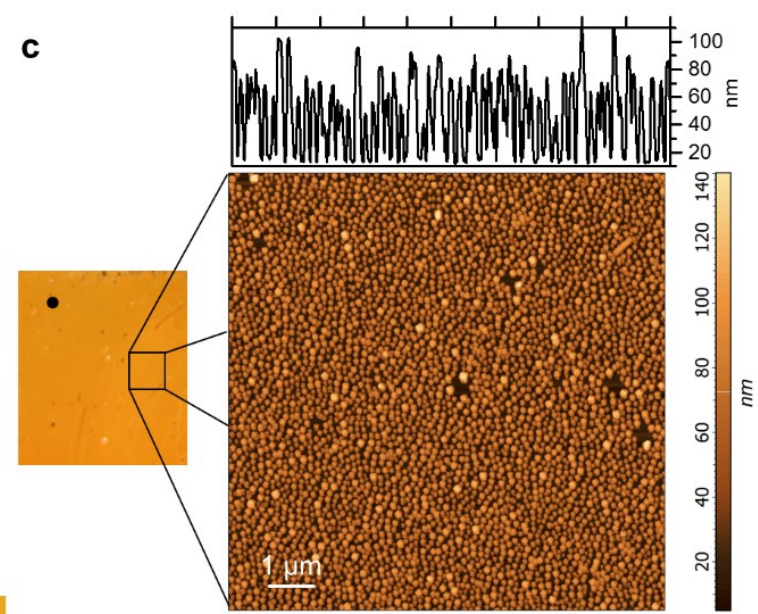

d
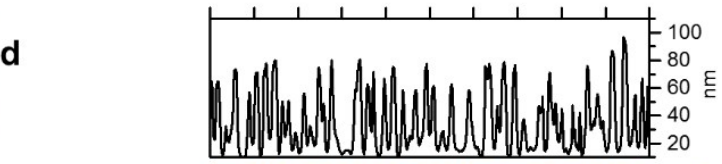

\section{.}

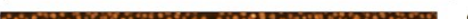

요

Figure 4.2. a) schematic demonstrating the fabrication process of the NPoM system, with a TEM image of the AgNC dropcast b) a sample of reflection spectra throughout the embedment process and the predicted colour by converting the spectrum to SRGB. c,d) photographs and associated AFM images of before and after embedment respectively. The embedment depth is measured to be $21 \pm 4 \mathrm{~nm}$

The full colour gamut of the system is predicted based off the reflectance spectra gathered in-situ during the embedment process by collecting reflectance spectra throughout the process and converting them to the CIE 1931 colour space (Figure 4.3). The system produces a range of red/orange colours. It should be noted that the calculated colours differ from the actual 
photographed colours (Figure 4.2c,d) since they are based solely off the reflectance spectrum, whereas the observed colours have a sizeable component of diffuse scattering. The light source used does not perfectly match the D65 illuminant used in the spectrum-colour conversion. With reflected light, the colour spectrum of the light source influences the perceived colour. The heating process can be stopped at any point, allowing samples with the intermediate colours to be produced. The temperature of the system is proportional to the rate of embedment allowing finer control over the process. ${ }^{30}$
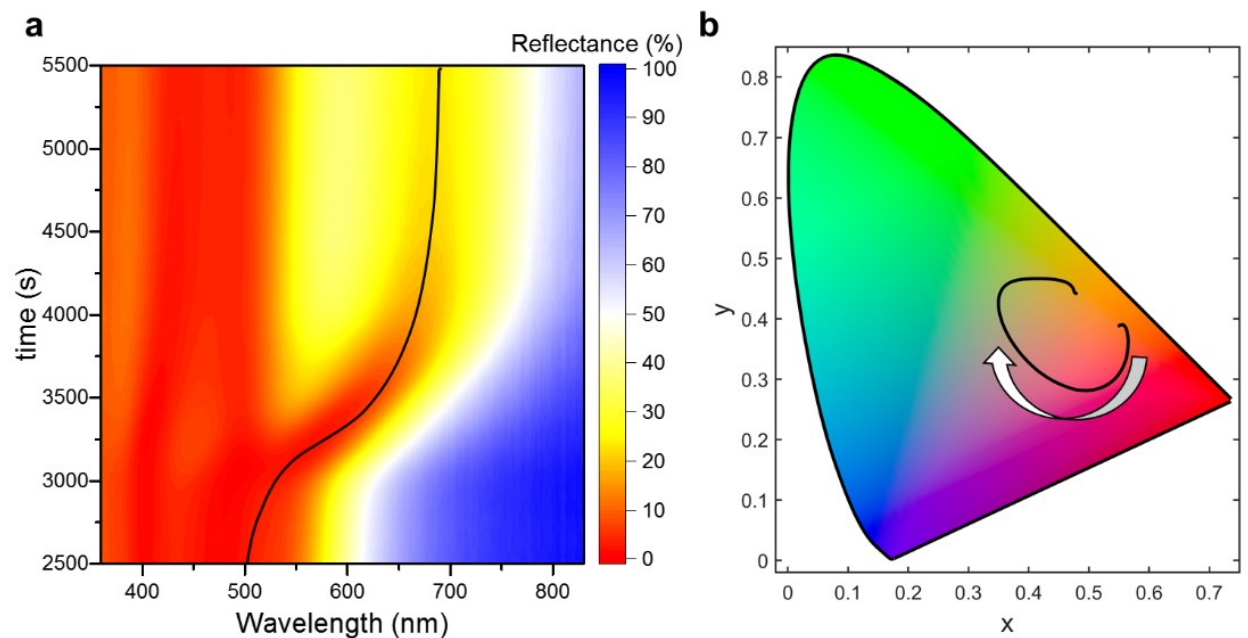

Figure 4.3 a) reflectance spectral colormap showing the embedment process as a function of time b) the chromaticity of the system expressed in the CIE 1931 colour space. A wide gamut is observed in the redgreen region.

The colours produced have a wide gamut, occupying a sizeable portion of the red-green colour space. However, the produced colours are limited due to the use of a gold back-reflector; all samples will have some yellow tint due to the interband transitions of Au occurring below $\sim 500 \mathrm{~nm}$ (Figure 4.3a). ${ }^{41}$ This prevents the production of colours on the blue end of the spectrum. Despite this the approach demonstrates a highly-tunable system to produce plasmon-derived colours, with the potential to expand the colour gamut by using alternative materials.

\subsubsection{Plasmonic colour generation in AgNC / Ag NPoM films}

Due to the limitations of colour generation when using Au as the plasmonic or backreflecting material, AgNC / Ag NPoM films were made to generate plasmon-derived colours in the same fashion. FDTD calculations predict the gap plasmon of the AgNC / Ag system to have greater tunability across the visible range Figure $4.4 a$. The plasmon is clearly distinguishable even at a 22 
$\mathrm{nm}$ gap distance can be red-shifted as far as in the case of the Au mirror; with a $5 \mathrm{~nm}$ gap, the plasmon is shifted beyond $700 \mathrm{~nm}$ as well. This potentially allows absorbance to be tuned as far as it was in the Au system, but with access to the bluer end of the system, giving greater tunability.
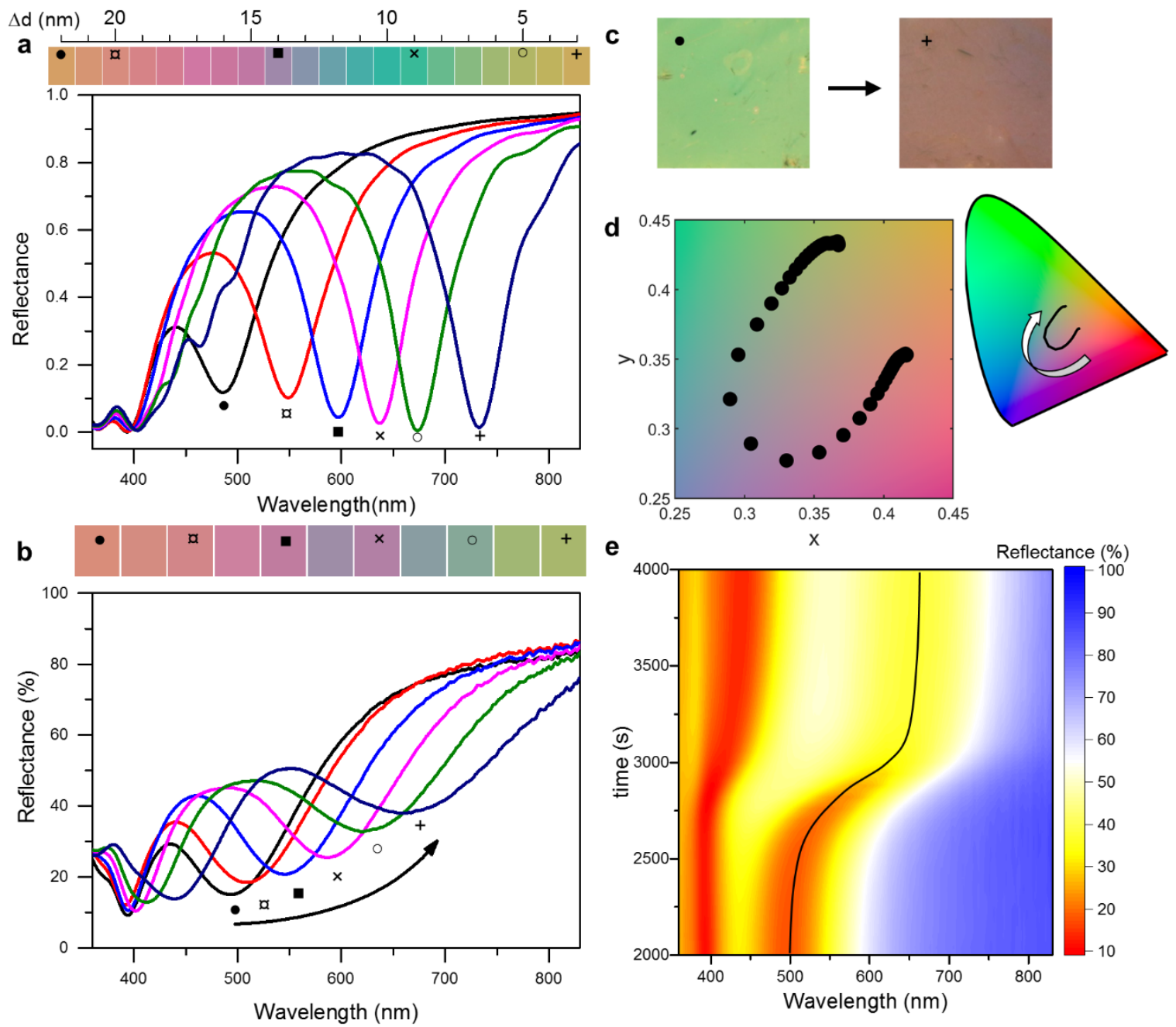

Figure 4.4. a) Predicted reflectance and colours from FDTD model consisting of a $20 \mathrm{~nm}$ PS film and b) sampled experimental spectra of and predicted colours of the embedment process. c) photographs of the sample before and after the embedment process d) chromaticity of the embedment process, showing the AgNC / Ag system occupies the central region of the colour space e) spectral colourmap of the embedment process showing a rapid shift of the gap mode from $\sim 500-675 \mathrm{~nm}$

The films were fabricated in the same manner as the Au films, but with some modifications.

De-wetting of the polystyrene was initially observed upon annealing the film leaving significant holes in the polymer. A further complication arose with control samples containing only Ag films which when heated to the temperatures associated with the annealing and embedment processes formation of large grains was observed increasing the roughness of the $\mathrm{Ag}$ film. ${ }^{42}$ This was in part alleviated by adding a $\sim 2 \mathrm{~nm} \mathrm{Al}_{2} \mathrm{O}_{3}$ layer over the $\mathrm{Ag}$ films by atomic layer deposition (ALD). The 
ALD procedure resulted in smoother PS films over the Ag, however the ALD process itself was still detrimental to the Ag films (Figure E.3).

The maximum of the resonance shifts from $495-675 \mathrm{~nm}$, becoming shallower and broader as the embedment depth increases (Figure 4.4b). This mirrors the peak shifts obtained in the case of $\mathrm{AgNC} / \mathrm{Au}$, though the produced colours are different due to the absence of absorbance from the Au interband transitions, resulting in an overall bluer hue. This also enabled the gap-plasmon resonance to be better resolved, being clearly distinguishable at all points. At high embedment depths the shifting of the higher order mode, presumed to be the quadrupolar mode, from $395 \mathrm{~nm}$ $-440 \mathrm{~nm}$

More significant broadening of the resonance was observed than in the AgNC/Au system. During the embedment process the PS films de-wetted from the surface, impeding precise determinations of the embedment depth (Figure 4.4c,d). The de-wetted film appears to preferentially be drawn towards the AgNCs; voids in the film after the thermal embedment do not contain any AgNCs. Combined with the increased roughness of the Ag films, this contributed to greater polydispersity in gap distances.
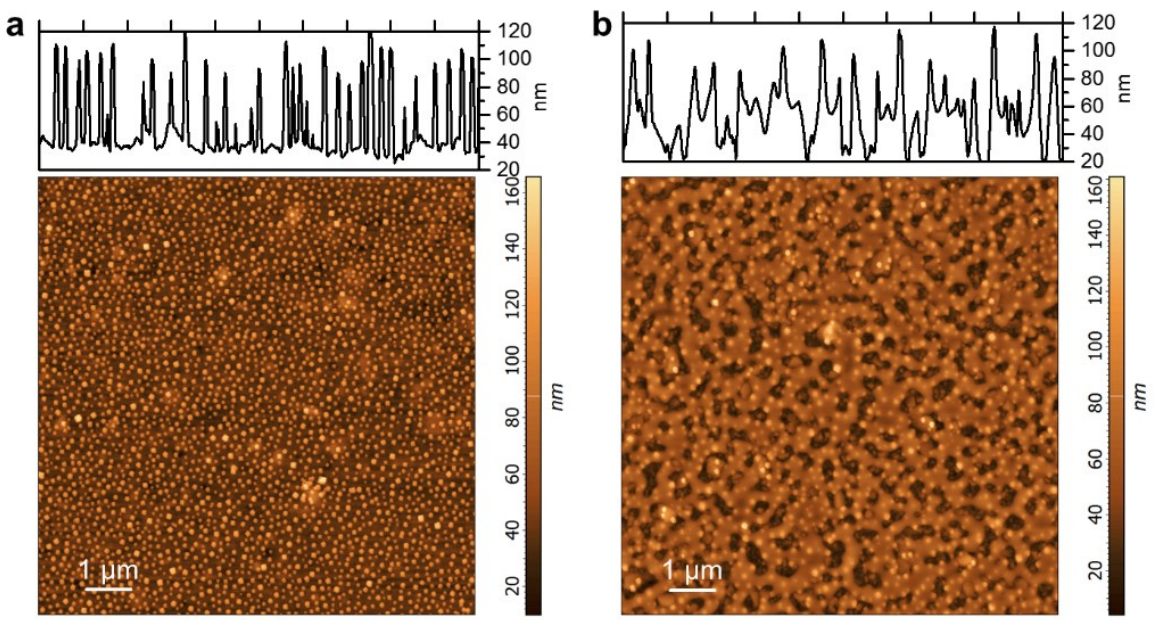

Figure 4.5. AFM topography measurements of the $\mathrm{Ag} / \mathrm{AgNC}$ sample a) before and $\mathbf{b}$ ) after embedment of the nanocubes. The thermal embedment process distorted the polymer films.

Nevertheless, a wide but distinct colour gamut was achieved with AgNC / Ag films. While the overall area of the colour space is not greater than that of the AgNC / Au films, the colours produced are distinct. The produced colours also lie in the of the CIE colour space around the achromatic point $(0.33,0.33)$ which is essential for producing a system which can display all primary 
colours (RGB). ${ }^{26}$ The use of silver nanocubes allows for greater sensitivity in an NPoM system due to the strong plasmon-hybridization. The use of similarly sized qausi-spherical silver nanoparticles does not shift the gap plasmon as strongly as silver nanocubes, accessing less of the colour space (Figure E.4).

Despite the ideal placement of the AgNC / Ag system in the colour space, a full range of primary colours is not produced. Band broadening hampers the strength of the produced colours; the obtained color gamut is not as wide as theoretically predicted (Figure E.5). The range is limited in blue end of the spectrum by interferences from the higher-order modes of the silver nanocubes which absorb around 400 - $500 \mathrm{~nm}$ (Figure 4.4e). Though not as severe as in the case of AgNC / Au films this still poses a limitation; non-overlapping, and distinct resonances are required to produce the widest range of plasmonic colours. Addressing the challenges in quality of the underlying Ag film and PS layer, as well as the dispersity of the colloidal silver nanocubes would help improve the system. Smaller silver nanocubes would potentially give more access for initial absorbances to be in the blue end of the spectrum, as would an initially thicker polystyrene film. (Figure E.6). This indicates there is yet greater potential in this system to be achieved which could enable the facile production of a wide gamut colour palette.

\subsubsection{Prospects for Photothermal Patterning}

The thermoplasmonic patterning procedure outlined in Chapter 3 can potentially be applied to this system. Figure 4.6 shows preliminary results involving a test pattern produced on a $\mathrm{Ag} / \mathrm{Au}$ NPoM film with $488 \mathrm{~nm}$ excitation. The test pattern produced in the film shows a strong contrast and is clearly distinguishable from the underlying substrate. (a), In the AFM topography the change is less apparent; the difference in NC height is hardly noticeable, demonstrating the high sensitivity of the system. Increasing exposure times and power produced larger spots in the substrate, with ablation of the central region occurring at high power. With finer control over the power delivered to the sample, it could be possible to print the full palette of colours in microscale, or potentially sub-diffraction limit patterning by exploiting the gaussian-shaped laser intensity. ${ }^{9}$ 


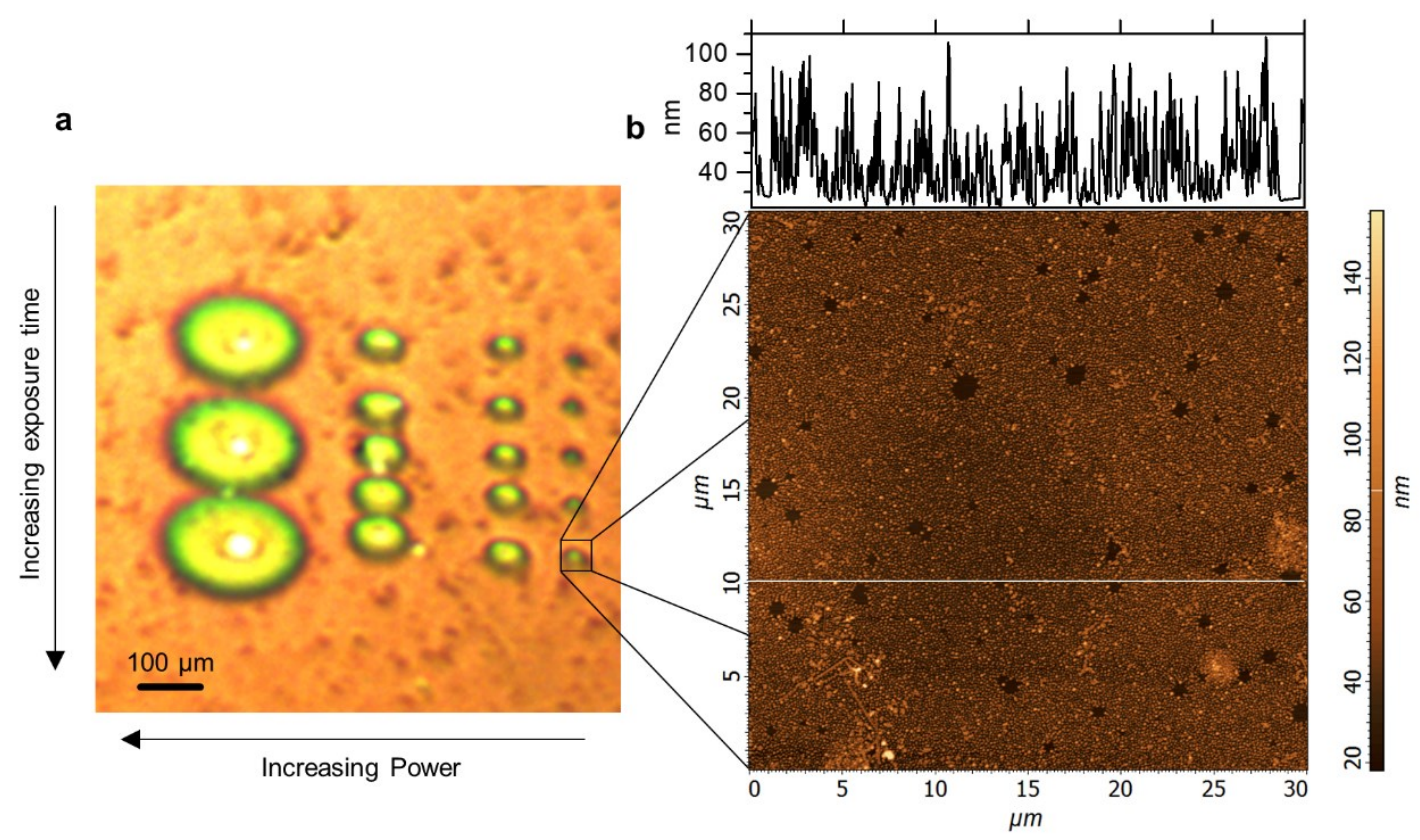

Figure 4.6 a) Photograph of reflected llight off of a test pattern produced by thermoplasmonic patterning. b) the change in AFM topography is hardly noticeable despite the clear contrast in the optical image. The cross section is given where the white line is shown.

\subsection{Conclusion.}

A simple, scalable, lithography-free approach to the generation of plasmonic colours is demonstrated using a AgNC NPoM system. The films are simple to fabricate, and plasmonic colours can be generated solely by heating, with relatively low temperature requirements. AgNC / $\mathrm{Au}$ and AgNC / Ag systems both displayed a strong gap-resonance which could be tuned between 500 - $700 \mathrm{~nm}$. Each system demonstrated a wide but distinct colour gamut, with AgNC/Au producing colours in the red-green colour space, and AgNC/Ag producing colours around the center of the colour space. The samples presented here were heated in bulk and produced centimeter-scale samples of single colours, but the embedment can easily be scaled down to microscale or nanoscale optical colour printing by utilizing thermoplasmonic patterning. Thermoplasmonic patterning allows for the rapid, localized heating of the nanostructures. By controlling the power of the laser delivered to the particle is potentially possible to carefully control the embedment depth, printing an entire palette of colours on-demand. Incorporating thermoplasmonic patterning to the system presented in this work would make the technique competitive with other methods for high resolution plasmonic colour patterning. 


\subsection{References}

(1) Rycenga, M.; Cobley, C. M.; Zeng, J.; Li, W.; Moran, C. H.; Zhang, Q.; Qin, D.; Xia, Y. Controlling the Synthesis and Assembly of Silver Nanostructures for Plasmonic Applications. Chem. Rev. 2011, 111 (6), 3669-3712.

(2) Mayer, K. M.; Hafner, J. H. Localized Surface Plasmon Resonance Sensors. Chem. Rev. 2011, 111 (6), 3828-3857.

(3) Blaber, M. G.; Arnold, M. D.; Ford, M. J. A Review of the Optical Properties of Alloys and Intermetallics for Plasmonics. J. Phys. Condens. Matter 2010, 22 (14), 143201.

(4) Freestone, I.; Meeks, N.; Sax, M.; Higgitt, C. The Lycurgus Cup - A Roman Nanotechnology. Gold Bull. 2007, 40 (4), 270-277.

(5) Colomban, P. The Use of Metal Nanoparticles to Produce Yellow, Red and Iridescent Colour, from Bronze Age to Present Times in Lustre Pottery and Glass: Solid State Chemistry, Spectroscopy and Nanostructure. J. Nano Res. 2009, 8, 109-132.

(6) Kristensen, A.; Yang, J. K. W.; Bozhevolnyi, S. I.; Link, S.; Nordlander, P.; Halas, N. J.; Mortensen, N. A. Plasmonic Colour Generation. Nat. Rev. Mater. 2016, 2 (1), 16088.

(7) Kumar, K.; Duan, H.; Hegde, R. S.; Koh, S. C. W.; Wei, J. N.; Yang, J. K. W. Printing Colour at the Optical Diffraction Limit. Nat. Nanotechnol. 2012, 7 (9), 557-561.

(8) Roberts, A. S.; Pors, A.; Albrektsen, O.; Bozhevolnyi, S. I. Subwavelength Plasmonic Color Printing Protected for Ambient Use. Nano Lett. 2014, 14 (2), 783-787.

(9) Zhu, X.; Vannahme, C.; Højlund-Nielsen, E.; Mortensen, N. A.; Kristensen, A. Plasmonic Colour Laser Printing. Nat. Nanotechnol. 2016, 11 (4), 325-329.

(10) Clausen, J. S.; Højlund-Nielsen, E.; Christiansen, A. B.; Yazdi, S.; Grajower, M.; Taha, H.; Levy, U.; Kristensen, A.; Mortensen, N. A. Plasmonic Metasurfaces for Coloration of Plastic Consumer Products. Nano Lett. 2014, 14 (8), 4499-4504.

(11) Greybush, N. J.; Charipar, K.; Geldmeier, J. A.; Bauman, S. J.; Johns, P.; Naciri, J.; Charipar, N.; Park, K.; Vaia, R. A.; Fontana, J. Dynamic Plasmonic Pixels. ACS Nano 2019, 13 (4), 3875-3883.

(12) Olson, J.; Manjavacas, A.; Basu, T.; Huang, D.; Schlather, A. E.; Zheng, B.; Halas, N. J.; Nordlander, P.; Link, S. High Chromaticity Aluminum Plasmonic Pixels for Active Liquid Crystal Displays. ACS Nano 2016, 10 (1), 1108-1117.

(13) Peng, J.; Jeong, H.-H.; Lin, Q.; Cormier, S.; Liang, H.-L.; De Volder, M. F. L.; Vignolini, S.; Baumberg, J. J. Scalable Electrochromic Nanopixels Using Plasmonics. Sci. Adv. 2019, 5 (5), eaaw2205.

(14) Kang, H.; Lee, J. W.; Nam, Y. Inkjet-Printed Multiwavelength Thermoplasmonic Images for Anticounterfeiting Applications. ACS Appl. Mater. Interfaces 2018, 10 (7), 6764-6771.

(15) Gutruf, P.; Zou, C.; Withayachumnankul, W.; Bhaskaran, M.; Sriram, S.; Fumeaux, C. Mechanically Tunable Dielectric Resonator Metasurfaces at Visible Frequencies. ACS Nano 2016, 10 (1), 133-141.

(16) Montelongo, Y.; Tenorio-Pearl, J. O.; Williams, C.; Zhang, S.; Milne, W. I.; Wilkinson, T. D. Plasmonic Nanoparticle Scattering for Color Holograms. Proc. Natl. Acad. Sci. U. S. A. 2014, 111 (35), 12679-12683.

(17) Duempelmann, L.; Gallinet, B.; Novotny, L. Multispectral Imaging with Tunable Plasmonic Filters. ACS Photonics 2017, 4 (2), 236-241.

(18) Stewart, J. W.; Akselrod, G. M.; Smith, D. R.; Mikkelsen, M. H. Toward Multispectral Imaging with Colloidal Metasurface Pixels. Adv. Mater. 2017, 29 (6). 
(19) Ho, J.; Dong, Z.; Daqiqeh Rezaei, S.; Ng, R. J. H.; H. H. Koay, E.; Ramakrishna, S.; Yang, J. K. W. Wide-Gamut Plasmonic Color Palettes with Constant Subwavelength Resolution. ACS Nano 2019.

(20) Tan, S. J.; Zhang, L.; Zhu, D.; Goh, X. M.; Wang, Y. M.; Kumar, K.; Quu, C.; Yang, J. K. W. Plasmonic Color Palettes for Photorealistic Printing with Aluminum Nanostructures. Nano Lett. 2014, 14 (7), 4023-4029.

(21) Cheng, F.; Gao, J.; Stan, L.; Rosenmann, D.; Czaplewski, D.; Yang, X. Aluminum Plasmonic Metamaterials for Structural Color Printing. Opt. Express 2015, 23 (11), 14552.

(22) Wang, L.; Ng, R. J. H.; Safari Dinachali, S.; Jalali, M.; Yu, Y.; Yang, J. K. W. Large Area Plasmonic Color Palettes with Expanded Gamut Using Colloidal Self-Assembly. ACS Photonics 2016, 3 (4), 627-633.

(23) Zhang, Y.; Zhang, Q.; Ouyang, X.; Lei, D. Y.; Zhang, A. P.; Tam, H.-Y. Ultrafast LightControlled Growth of Silver Nanoparticles for Direct Plasmonic Color Printing. ACS Nano 2018, 12 (10), 9913-9921.

(24) Guay, J. M.; Calà Lesina, A.; Côté, G.; Charron, M.; Poitras, D.; Ramunno, L.; Berini, P.; Weck, A. Laser-Induced Plasmonic Colours on Metals. Nat. Commun. 2017, 8 (May).

(25) Roberts, A. S.; Novikov, S. M.; Yang, Y.; Chen, Y.; Boroviks, S.; Beermann, J.; Mortensen, N. A.; Bozhevolnyi, S. I. Laser Writing of Bright Colors on Near-Percolation Plasmonic Reflector Arrays. ACS Nano 2019, 13 (1), 71-77.

(26) Miyata, M.; Hatada, H.; Takahara, J. Full-Color Subwavelength Printing with GapPlasmonic Optical Antennas. Nano Lett. 2016, 16 (5), 3166-3172.

(27) Baumberg, J. J.; Aizpurua, J.; Mikkelsen, M. H.; Smith, D. R. Extreme Nanophotonics from Ultrathin Metallic Gaps. Nat. Mater. 2019.

(28) Moreau, A.; Ciracì, C.; Mock, J. J.; Hill, R. T.; Wang, Q.; Wiley, B. J.; Chilkoti, A.; Smith, D. R. Controlled-Reflectance Surfaces with Film-Coupled Colloidal Nanoantennas. Nature 2012, 492 (7427), 86-89.

(29) Bottomley, A.; Prezgot, D.; Coyle, J. P.; lanoul, A. Dynamics of Nanocubes Embedding into Polymer Films Investigated via Spatially Resolved Plasmon Modes. Nanoscale 2016, 8 (21), 11168-11176.

(30) Bushell, M.; Bottomley, A.; lanoul, A. Utilization of Hybrid Plasmonic Modes to Investigate Surface Interactions between Nanocubes and Polymer Substrates. Appl. Phys. A Mater. Sci. Process. 2017, 123 (2), 1-7.

(31) Skrabalak, S. E.; Au, L.; Li, X.; Xia, Y. Facile Synthesis of Ag Nanocubes and Au Nanocages. Nat. Protoc. 2007, 2 (9), 2182-2190.

(32) Vianna, S. D. B.; Lin, F. Y.; Plum, M. A.; Duran, H.; Steffen, W. Dynamics of Ultra-Thin Polystyrene with and without a (Artificial) Dead Layer Studied by Resonance Enhanced Dynamic Light Scattering. J. Chem. Phys. 2017, 146 (20).

(33) Hall, D. B.; Underhill, P.; Torkelson, J. M. Spin Coating of Thin and Ultrathin Polymer Films. Polym. Engeneering Sci. 1998, 38 (12), 2039-2045.

(34) Palik, E. D. Handbook of Optical Constants of Solids; 1985; Vol. 1.

(35) Sultanova, N.; Kasarova, S.; Nikolov, I. Dispersion Properties of Optical Polymers. Acta Phys. Pol. A 2009, 116 (4), 585-587.

(36) König, T. A. F.; Ledin, P. A.; Kerszulis, J.; Mahmoud, M. A.; El-Sayed, M. A.; Reynolds, J. R.; Tsukruk, V. V. Electrically Tunable Plasmonic Behavior of Nanocube-Polymer Nanomaterials Induced by a Redox-Active Electrochromic Polymer. ACS Nano 2014, 8 (6), 6182-6192. 
(37) Johnson, P. B.; Christy, R. W. Optical Constants of the Noble Metals. Phys. Rev. B 1972, 6 (12), 4370-4379.

(38) Le, F.; Lwin, N. Z.; Steele, J. M.; Käll, M.; Halas, N. J.; Nordlander, P. Plasmons in the Metallic Nanoparticle-Film System as a Tunable Impurity Problem. Nano Lett. 2005, 5 (10), 2009-2013.

(39) Zhang, S.; Bao, K.; Halas, N. J.; Xu, H.; Nordlander, P. Substrate-Induced Fano Resonances of a Plasmonic Nanocube: A Route to Increased-Sensitivity Localized Surface Plasmon Resonance Sensors Revealed. Nano Lett. 2011, 11 (4), 1657-1663.

(40) Acharya, S.; Hill, J. P.; Ariga, K. Soft Langmuir-Blodgett Technique for Hard Nanomaterials. Adv. Mater. 2009, 21 (29), 2959-2981.

(41) West, P. R.; Ishii, S.; Naik, G. V.; Emani, N. K.; Shalaev, V. M.; Boltasseva, A. Searching for Better Plasmonic Materials. Laser Photonics Rev. 2010, 4 (6), 795-808.

(42) Kim, H. C.; Alford, T. L.; Allee, D. R. Thickness Dependence on the Thermal Stability of Silver Thin Films. Appl. Phys. Lett. 2002, 81 (22), 4287-4289.

(43) Xue, J.; Zhou, Z.-K.; Wei, Z.; Su, R.; Lai, J.; Li, J.; Li, C.; Zhang, T.; Wang, X.-H. Scalable, Full-Colour and Controllable Chromotropic Plasmonic Printing. Nat. Commun. 2015, 6 (May), 8906. 


\section{Chapter 5: UV plasmonic materials: Investigating Indium and Aluminium Nanocrystals for Deep-UV Surface-Enhanced Raman Spectroscopy}

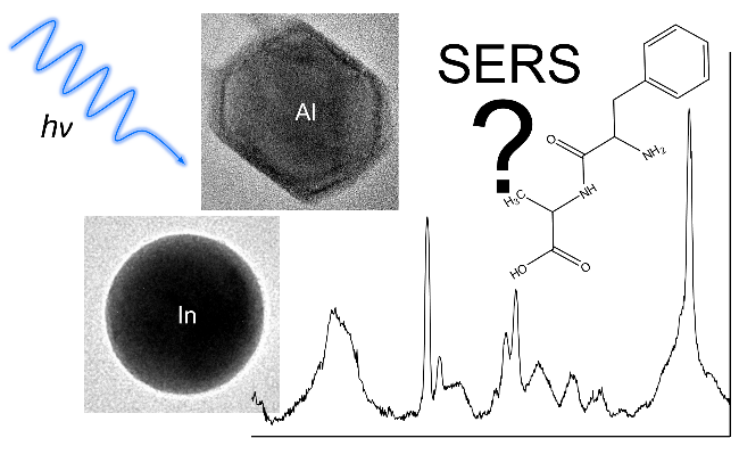

\section{Abstract}

Plasmonic materials are typically implemented in the visible and near-infrared spectral domains, however extending plasmonic applications into the ultra-violet is of major interest in sensing and catalysis. One such application is the extension of surface-enhanced Raman spectroscopy (SERS) to the deep-UV (DUV) region of the electromagnetic spectrum. This is regarded as an important milestone with potential to further increase the applicability with SERS particularly with respect the study of biological molecules. In this work indium and aluminium nanocrystals are synthesized as UV-plasmonic materials and potential platforms for DUV-SERS. Raman measurements were performed at $229 \mathrm{~nm}$, target molecules include direct functionalization of the nanocrystals with phenyltrimethoxysilane (PhTMS), and alanine-phenylalanine as a model peptide. While no appreciable DUV-SERS enhancement was observed, finite-difference timedomain modelling suggest there is potential of aluminium nanocrystals in DUV-SERS and other UV-plasmonic applications. 


\subsection{Introduction}

Plasmonic materials offer a unique route towards the manipulation of light. They can efficiently absorb and scatter light and create intense local electric fields at the surface, essentially concentrating light at the nanoscale. This has enabled their use in applications such as light harvesting, ${ }^{1}$ photodetection, ${ }^{2}$ catalysis, ${ }^{3}$ and sensing. ${ }^{4}$ Plasmonics has mostly been applied to the visible and near-infrared portions of the electromagnetic spectrum, but extension of plasmonics to the ultraviolet (UV) spectral domain is of major interest for catalytic ${ }^{5}$ and sensing applications. ${ }^{6} \mathrm{UV}$ plasmonic materials represent a new class of high-energetic plasmonic materials which may yield unique light-matter interactions. ${ }^{7}$

One area in which UV-plasmonics can be applied is to surface-enhanced Raman scattering (SERS). SERS is a powerful analytical technique and phenomenon which primarily originates from the electric field enhancement which occurs at the surface of metal nanomaterials in response to the excitation of a localized surface plasmon resonance (LSPR). ${ }^{8}$ The SERS effect allows for the enhancement of Raman spectroscopy by many orders of magnitude (typical SERS enhancement factors range from $10^{4}-10^{6}$ ) and is capable of limits of detection down to the single-molecule level. ${ }^{9,10}$ Since the phenomenon's first observation over 40 years ago, ${ }^{11}$ SERS has matured into a large field of research and a versatile analytical technique which has been applied to numerous areas such as in biomedical, ${ }^{12}$ environmental, ${ }^{13}$ or forensic applications. ${ }^{14}$ Instrumentation can be used to implement SERS in a variety of fashions, from research-focused instruments capable of hyperspectral imaging ${ }^{15}$ or tip-enhanced scanning, ${ }^{16}$ to simple point-of-care devices which offer ease-of-use and can be operated with minimal training. ${ }^{17}$

Research and implementation of SERS has primarily focused on using visible or near-IR (NIR) wavelength excitation, partly due to the ease and availability of the instrumentation (visible and NIR lasers) but also since the most prevalent plasmonic materials, $\mathrm{Ag}$ and $\mathrm{Au}$, only support plasmon resonances in the visible range of the EM spectrum. ${ }^{18}$ Another region of the EM spectrum in which it is useful to perform Raman, is in the deep-UV (DUV, 190 - $300 \mathrm{~nm}$ ) region. DUV Raman spectroscopy is desirable as many molecules absorb strongly in the UV region thus resonanceRaman scattering (RRS) effects become commonplace; when the Raman excitation wavelength 
falls within an electronic absorption band of the analyte enhancement of the Raman signal by 2 to 5 orders of magnitude can be observed. ${ }^{19}$ With excitation below $250 \mathrm{~nm}$ many molecules will demonstrate RRS while minimizing the chances for fluorescent backgrounds. DUV Raman spectroscopy also is uniquely capable of gaining insight into structure dynamics in biomolecules such as peptides and proteins by targeting the amide peptide bonds for RRS spectra. ${ }^{20}$ DUV SERS has strong potential for applications such as explosives detection ${ }^{21}$ and protein structure dynamics. ${ }^{22}$

Realising deep-UV SERS requires the implimentation of new plasmonic materials; the strongest-enhancing and most prevalent metals for SERS, Ag and Au are not suitable for DUV plasmonics. These metals have high imaginary dielectric constants in the DUV region of the spectrum; their spectral response is dominated by the absorption in interband transitions, not surface plasmons. ${ }^{18}$ Other suitable materials are thus required to act as SERS substrates. Theoretical alternatives which have appropriate dielectric functions include Al, Ga, In, Sn, Ru, and Rh. ${ }^{18,23}$ UV SERS has been reported most commonly using Al, ${ }^{6,24-26}$ but also with In, ${ }^{27} \mathrm{Ga},{ }^{28} \mathrm{Rh},{ }^{29}$ and other materials such as alloys. ${ }^{30}$ However these metals do not boast the high quality LSPR's of $\mathrm{Ag}$ and $\mathrm{Au}$, and demonstrate much lower extinction cross-sections. In most cases the reported enhancement factors are limited, ranging from $\sim 10^{1}-10^{3}$.

This work aims to develop investigate the use of colloidal indium and aluminum nanoparticles for DUV-SERS. Indium and aluminum were selected based off of their predicted high quality LSPR's in the desired spectral region, ${ }^{18,31}$ and the availability of facile methods for their colloidal synthesis. ${ }^{32,33}$ Strategies for measuring DUV Raman, synthesizing, labelling and assembling the particles are explored. While the observed SERS enhancement in this work is minimal, the work explores the synthesis and properties of UV plasmonic nanocrystals. FDTD calculations are also used to support experimental observations, as well as suggest the potential for further incorporation of hybridized plasmon modes can be important towards the realization of DUV-SERS. 


\subsection{Experimental}

Materials. Indium chloride ( $\mathrm{InCl}_{3}$ 99.99\%, anhydrous, Sigma), Sodium citrate dibasic $\left(\mathrm{Na}_{2} \mathrm{Cit} \cdot\right.$ 1.5 $\mathrm{H}_{2} \mathrm{O}$, Sigma), Diethylene glycol (DEG, 99\%, Anhydrous), M N,N-dimethylethylamine alane (0.5 M in toluene, Sigma), titanium (IV) isopropoxide (Sigma, 97\%)

Synthesis of In nanoparticles. To a triple-neck round bottom flask, $138 \mathrm{mg}(0.625 \mathrm{mmol})$ of $\mathrm{InCl}_{3}$, $131 \mathrm{mg}(0.500 \mathrm{mmol})$ of $\mathrm{Na}_{2} \mathrm{Cit} \cdot 1.5 \mathrm{H}_{2} \mathrm{O}$ were added to $25 \mathrm{~mL}$ of DEG. The flask was placed in an oil bath and heated to $100{ }^{\circ} \mathrm{C}$ under magnetic stirring under constant $\mathrm{N}_{2}$ flow with an outlet fed through an oil bubbler. $1 \mathrm{~mL}$ of $\mathrm{NaBH}_{4}$ solution was prepared in $\mathrm{diH}_{2} \mathrm{O}$, containing a molar ratio of $\mathrm{InCl}_{3}: \mathrm{NaBH}_{4}$ between 1:2 and 1:6. After $\sim 1 \mathrm{~h}$, after the reagants had dissolved, $1 \mathrm{~mL}$ of $\mathrm{NaBH}_{4}$ solution was added. After $\sim 15$ minutes the flask was removed from the bath and cooled in an icewater bath. The resultant solution was centrifuged 3 times at 12000 RCF and re-dispersed in $4 \mathrm{~mL}$ of anhydrous ethanol.

Conjugation with Phenyltrimethoxysilane. InNP's were centrifuged and re-dispersed in $2 \mathrm{~mL}$ of a $1 \%$ methanolic solution of phenyltrimethoxysilane (PhTMS). The solution was allowed to sit for $1 \mathrm{~h}$ before being centrifuged and rinsed 3 times at $12000 \mathrm{RCF}$ and then re-dispersed in $2 \mathrm{~mL}$ of methanol.

Synthesis of core-shell In@SiO 2 . To make core-shell In@SiO 2 nanoparticles a modified Stöber process developed by Bourdeau et al. was used. ${ }^{34}$ To a $15 \mathrm{~mL}$ conical tube, $500 \mu \mathrm{L}$ of $\mathrm{nn} \mathrm{NP}$ solution was added, followed $1914 \mu \mathrm{L}$ of $\mathrm{H}_{2} \mathrm{O}, \sim 8 \mathrm{~mL}$ of EtOH and between $11-88 \mu \mathrm{L}$ of TEOS was added. The solutions were stirred for 15 minutes, after which $86 \mu \mathrm{L}$ of $35 \% \mathrm{NH}_{4} \mathrm{OH}$ was added and allowed to stir for $16 \mathrm{~h}$. The solution was centrifuged 3 times at $12000 \mathrm{RCF}$ and re-dispersed in $4 \mathrm{~mL}$ of anhydrous ethanol.

Langmuir-Blodgett Monolayers. InNP were re-dispersed in $300 \mu \mathrm{L}$ of $\mathrm{ChCl}_{3}$ and then deposited onto the surface of a NIMA 311D Langmuir trough filled with deionized water using a microsyringe. Quartz slides were raised out of the trough at $2 \mathrm{~mm} / \mathrm{min}$ to transfer the Langmuir films onto the substrate.

Synthesis of Al nanoparticles. This reaction was performed in a dry box under $\mathrm{N}_{2}$ atmosphere. 25 $\mathrm{mL}$ of dioxane was added to a $100 \mathrm{~mL}$ pressure flask. $6.5 \mathrm{~mL}$ of $0.5 \mathrm{M} \mathrm{N}, \mathrm{N}$-dimethylethylamine 
alane in toluene was added, followed by $0.5 \mathrm{~mL}$ of a $3.3 \mathrm{mM}$ solution of titanium (IV) isopropoxide in toluene after 2 minutes. After a further 2 minutes, $0.5 \mathrm{~mL}$ of $250 \mathrm{mM}$ oleic acid in dioxane was added. The pressure flask was sealed and removed from the dry box, then heated at $40^{\circ} \mathrm{C}$ for $2 \mathrm{~h}$. The solution slowly transitioned to a dark brown then after $2 \mathrm{~h}$ the reaction was heated to $60^{\circ} \mathrm{C}$ and allowed to proceed for a further 45 min until a dark grey solution was obtained. The reaction was quenched in an ice water bath. The resultant solution was centrifuged 3 times at $12000 \mathrm{RCF}$ and re-dispersed in $8 \mathrm{~mL}$ of anhydrous ethanol. Aluminium nanocrystals were deposited onto quartz substrates by dynamic spin-coating. Up to $1 \mathrm{~mL}$ of an ethanolic suspension of AINP was deposited dropwise on to a quartz substrate spinning at $4000 \mathrm{rpm}$ via a syringe.

UV Raman Spectroscopy. UV Raman spectroscopy was performed using a Coherent Innova 90c FreD Ar ion laser, with the $458 \mathrm{~nm}$ line frequency doubled to $229 \mathrm{~nm}$ with a $\beta_{\mathrm{BaBO}}$ crystal by second-harmonic generation. The laser was focused onto the sample in an almost-backscattering geometry. Solution spectra were taken in $1 \mathrm{~mL}$, cuvette with a $1 \mathrm{~cm}$ path length equipped with a magnetic stirrer. For solutions on solid-supported samples, spectra were taken by first dropping $100 \mu \mathrm{L}$ of solution to the sample then pressing the window of a demountable, low pathlength $(0.1$ $\mathrm{mm}$ ) quartz cuvette onto the surface. Accumulations were between 1 second and 3 minutes in length depending on conditions and signal strength.

\subsection{Results and Discussion}

\subsubsection{Synthesis of Indium nanoparticles.}

Indium nanoparticles were synthesized according to the citrate-assisted polyol method outlined by the procedure in section 5.2.32 $\mathrm{InCl}_{3}$ served as the indium source, $\mathrm{NaBH}_{4}$ the reducing agent, disodium citrate as the capping agent and diethylene glycol (DEG) as the high-temperature solvent. Control of the nanoparticle size can be achieved by adjusting the $\operatorname{lnCl} 3: \mathrm{NaBH}_{4}$ ratio; reducing the amount of $\mathrm{NaBH}_{4}$ increases the average resultant nanoparticle size. ${ }^{32}$ Figure 5.1 displays the UV-Vis spectrum and TEM images of a synthesis at a 1:5 $\operatorname{lnCl}_{3}: \mathrm{NaBH}_{4}$ ratio. The nanoparticles have an average size of $62 \pm 14 \mathrm{~nm}$ and display a broad UV-Vis extinction at 310 $\mathrm{nm}$. 
a

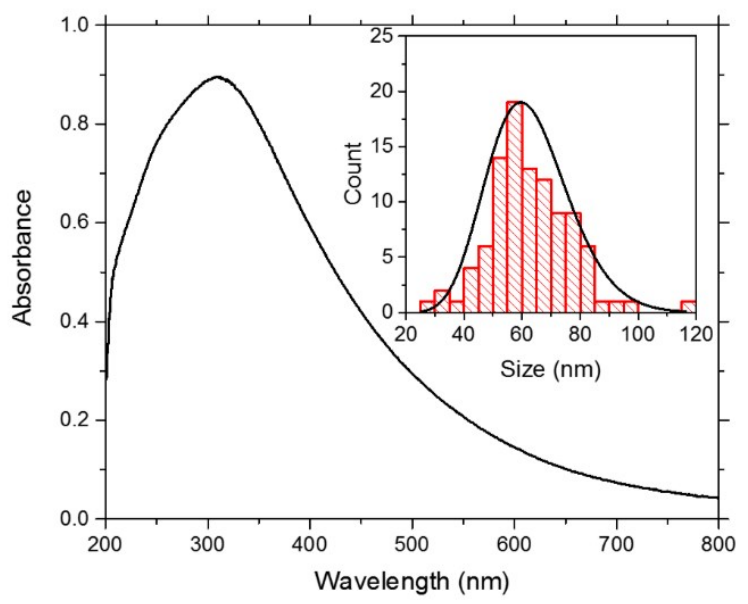

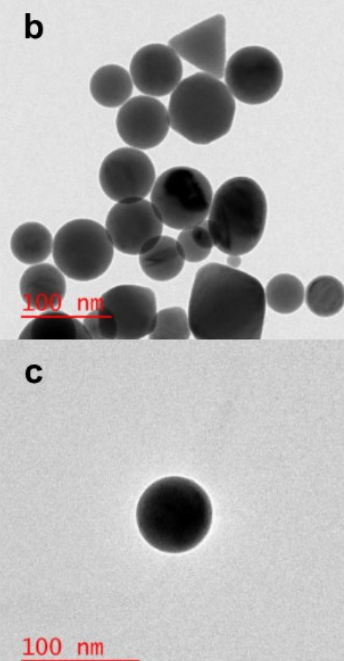

$100 \mathrm{~nm}$

Figure 5.1. a) UV-Visible spectrum of synthesized indium nanoparticles in $\mathrm{H}_{2} \mathrm{O}$, inset with a histogram $(n=100)$ showing a size distribution of $62 \pm 14 \mathrm{~nm}$. b) Transmission electron micrograph of the as-synthesized particles. Particles are largely spherical but with some polydispersity in shape c) Most of the particles are remarkably spherical and non-faceted for nanoparticles of this scale.

The produced particles are somewhat polydisperse in size, with a standard deviation of $22 \%$ of the mean size. In shape they are mostly spherical, though some polyhedral and plates are observed as well. It is worth noting that the spherical particles produced by this method are remarkably round; typical nanocrystals of this size made from other materials tend to be faceted as this produces the lowest surface-energy shape. ${ }^{35,36}$ Most of the observed In particles however appear to be perfectly spherical in shape. This occurs because the particles are believed to form in the molten state. ${ }^{32}$ This can be the case due to the relatively low melting point of bulk In (156 $\left.{ }^{\circ} \mathrm{C}\right)$, combined with the phenomenon of melting point depression in metal nanoparticles due to the increased proportion of surface atoms. ${ }^{37}$ The melting point of indium can be depressed to room temperature in the case of small $(<10 \mathrm{~nm})$ particles, ${ }^{38,39}$ and it is entirely reasonable they remain molten during the synthesis (performed at $>100{ }^{\circ} \mathrm{C}$ ) or at the very least during the formation of the seed particles. Thus, during formation of the nanocrystal the atoms are sufficiently mobile to allow a sphere to be the lowest energy state.

While the particles are synthesized under an $\mathrm{N}_{2}$ atmosphere, they are eventually exposed to ambient conditions leading to the formation of a $\ln _{2} \mathrm{O}_{3}$ shell. ${ }^{40}$ This native oxide coating was found to be about $\sim 3 \mathrm{~nm}$ thick by transmission electron microscopy (Figure F.1). The oxide coating has important ramifications with respect to SERS: it determines the surface-chemistry of the 
nanoparticles and sets a minimum distance any given Raman reporter must be from the metal nanoparticle surface. ${ }^{31}$

\subsubsection{Deep-UV SERS on In nanoparticles}

Two primary reporters were chosen as SERS tags for DUV-SERS experiments. L-Alaninephenylalanine (Ala-Phe) was chosen as a dipeptide, non-specific reporter as it contains a phenyl group which displays resonant-Raman enhancement, in addition to having an amide peptide bond. Detection of the amide bond would have demonstrated the applicability of DUV-SERS towards biomolecule structural characterisation. Phenyltrimethoxy silane (PhTMS) was chosen as a specific-binding analyte. This label contains contains a phenyl group for resonant-raman detection and the trimethoxy-silane linkage for bonding to oxide surface via condensation of the methoxy groups to surface hydroxyls. ${ }^{41}$ In this case the label forms a self-assembled monolayer (SAM) on the particle surface, maximizing the chances of the analyte experiencing EM enhancement from the nanoparticle. The solution UV resonance Raman spectra of the of $1 \% \mathrm{v} / \mathrm{v} \mathrm{PhTMS}$ in $\mathrm{MeOH}$, and $10 \mathrm{mM}$ Ala-Phe in $\mathrm{H}_{2} \mathrm{O}$ are displayed in Figure 5.2.
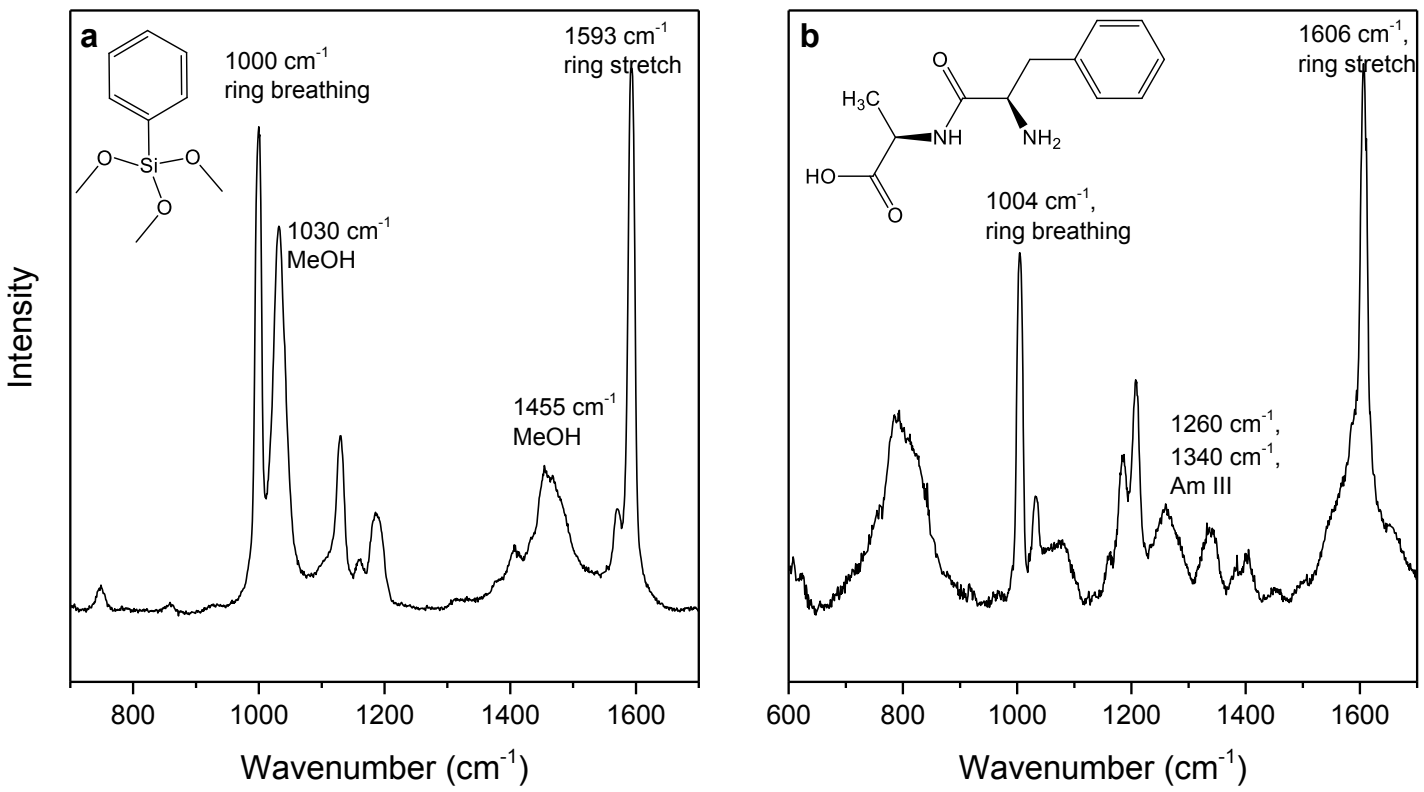

Figure 5.2. UV resonance-raman spectra of the primary Raman reporters used in this work a) a $1 \%$ solution of phenyltrimethoxysilane (PhTMS) in methanol and b) A $1 \mathrm{mM}$ Alanine-Phenylalanine solution in $\mathrm{H}_{2} \mathrm{O}$. Both present strong resonances from their phenyl constituents at $\sim 1000 \mathrm{~cm}^{-1}$ and $1606 \mathrm{~cm}^{-1}$. Two modes from the amide bond in Ala-Phe, AmIII 3 , Amll 2 , are assigned at $1260 \mathrm{~cm}^{-1}$ and $1340 \mathrm{~cm}^{-1}$ respectively. The Aml and Amll modes occur between $\sim 1550-1650 \mathrm{~cm}^{-1}$ are obscured by the strong ring stretching vibration at 1606 $\mathrm{cm}^{-1}$. 


\subsubsection{Phenyltrimethoxysilane}

Colloidal In nanoparticles conjugated with PhTMS were subsequently measured by UV Raman spectroscopy in methanolic and aqueous suspensions. Particle concentrations were adjusted to have a maximum extinction of $\sim 0.5-1.0$. Measurements were done in solution under magnetic stirring to avoid strong detection of photodecomposition products. No detectable signal of either the $1000 \mathrm{~cm}^{-1}$ or $1153 \mathrm{~cm}^{-1}$ modes was observed. The possible reasons for this would either be: insufficient Raman enhancement or the lack of binding of the analyte to the $\ln _{2} \mathrm{O}_{3}$ surface. In order test the latter the In nanoparticle surface was modified to make certain PhTMS was present.

\subsubsection{Silica shell growth on Indium particles}

In order to facilitate binding of the phenyltrimethoxysilane label core-shell In@ $\mathrm{SiO}_{2}$ nanoparticles were synthesized. Silane reactivity with $\mathrm{SiO}_{2}$ surfaces is well established, ${ }^{42}$ thus acted as a control sample to test both whether PhTMS was failing to bind to $\ln _{2} \mathrm{O}_{3}$ and to potentially provide a distance-dependence investigation of the DUV-SERS response. In@SiO 2 nanoparticles were synthesized using the well-established Stöber process. ${ }^{34,43}$ This process uses the ammoniumcatalyzed hydrolysis of tetra-ethyl orthosilicate (TEOS) to grow $\mathrm{SiO}_{2}$ particles, which can use existing particles as nucleation points to grow core-shell particles. The full procedure is outlined in 5.2. The aim was to produce very thin shells onto which labels can be deposited via tri-methoxy silanes. Figure 5.3 displays shells produced using $11-88 \mu \mathrm{L}$ of TEOS. 

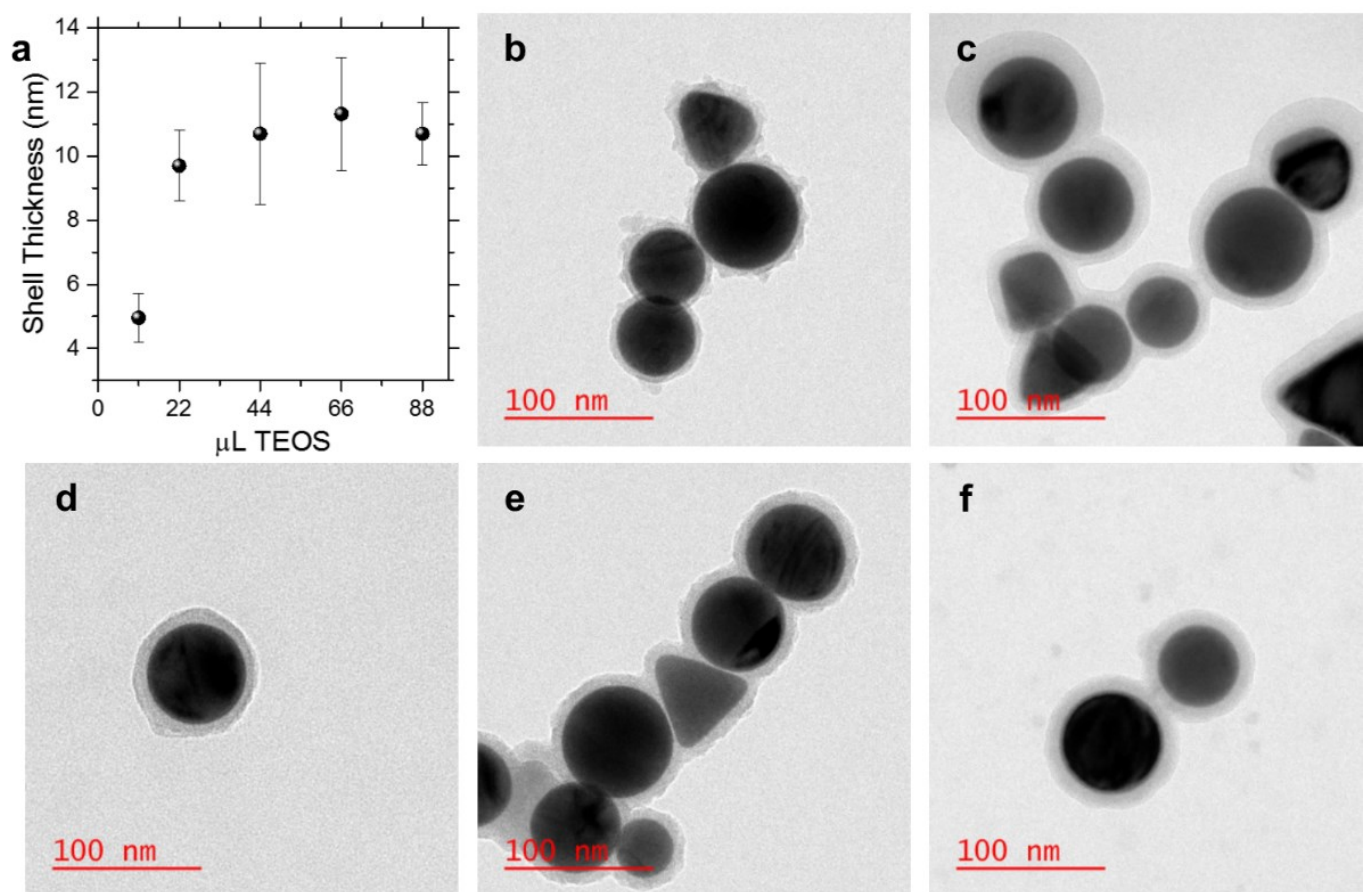

Figure 5.3. a) Shell thicknesses for different amounts of $10 \% \mathrm{v} / \mathrm{v}$ TEOS added to the shell growth process. Shell thicknesses between 5-11 nm were obtained. $\mathbf{b}-\mathbf{f}$ ) TEM images of shells grown with 11, 22, 44, 66, and $88 \mu \mathrm{L}$ of TEOS respectively. The average shell thickness did not vary greatly between $22-88 \mu \mathrm{L}$ of TEOS

Conformal $\mathrm{SiO}_{2}$ shells were produced around the In nanoparticles ranging between $5-11$ $\mathrm{nm}$. The average shell thickness did not vary greatly between $22-88 \mu \mathrm{L}$ of TEOS, indicating limited control over the thickness using this factor. Thickness can also be controlled by varying the quantity of catalyst $\left(\mathrm{NH}_{4} \mathrm{OH}\right)$ rather than TEOS. ${ }^{34}$ However, since the goal was to produce shells as thin as possible this was not explored in further detail. The initial particle concentration during the process had to be controlled as overgrowth of the shells fused neighbouring particles, causing excess aggregation. This can be observed in Figure 5.3 b,c and e, for example.

Even with the thinnest $\mathrm{SiO}_{2}$ shells, PhTMS was still not detectable by DUV Raman spectroscopy on the surface of InNP. Curiously, a strong mode is observed at $815 \mathrm{~cm}^{-1}$ (Figure F.2). The exact nature of this mode is not certain but is believed to be $\mathrm{SiO}_{4}$ or $\mathrm{SiOC}$ stretch, ${ }^{44,45}$ though this mode is typically weak under normal Raman conditions it may be enhanced by SERS or resonant-Raman effects. However, without a valid reference this mode is not valuable as it cannot be used to calculate the SERS enhancement and does not represent a potentially viable analyte. 


\subsubsection{Alanine-Phenylalanine}

Since the goal of the work was to demonstrate biomolecule detection at deep-UV wavelengths, alanine-phenylalanine was a more suitable choice as a model analyte. This molecule does not bind directly to the nanoparticles but exists free in solution, thus solution phase measurements are unlikely to display Raman enhancement. To avoid photodecomposition hindering measurements a hybrid liquid-film over solid support approach is used. ${ }^{24}$ Directly measuring solid-samples poses a challenge in DUV Raman spectroscopy, as the high-energy photons rapidly induce decomposition of most molecules. ${ }^{24} \mathrm{~A}$ film of InNP was deposited on a 2.5 $\times 2.5 \mathrm{~mm}$ quartz slide, over which the window component of a $0.1 \mathrm{~mm}$ path-length demountable cuvette is placed (Figure F.3). This maintains a liquid film where the analyte is free to flow allowing long accumulations with minimal photodecomposition. The disadvantage is that the majority of the analyte is not adsorbed to the surface and thus the measured signal may not a result of Raman enhancement. If a significant enhancement in Raman scattering exists though a quantifiable increase in signal would still be observable; even under typical SERS conditions a minority of the target molecules contribute to the majority of the signal. ${ }^{46}$ To make a 2-dimensional layer of In particles films were produced at an air-water interface and then transferred onto the surface of quartz substrates by the Langmuir-Blodgett technique. The produced films displayed linear chains of InNP when deposited at low surface pressures, and larger aggregates at high surface pressures(Figure F.5) .

UV Raman measurements were performed for $1 \mathrm{mM}$ and $10 \mathrm{mM}$ solutions of Ala-Phe over the deposited InNP films and over a blank quartz slide for reference. Figure 5.4 displays the average UV Raman spectrum obtained over one of the Langmuir-Blodgett films of InNP's. The ring modes $\left(1004 \mathrm{~cm}^{-1}\right.$ and $\left.1606 \mathrm{~cm}^{-1}\right)$ are readily apparent as well as a strong background signal from the fused quartz substrate and cuvette window. The $1004 \mathrm{~cm}^{-1}$ mode was used to quantify the Raman intensity (Figure 5.4 inset). Spectra acquired over InNP and over regular quartz slides proved to yield statistically indifferent intensities; no SERS enhancement could be quantified. Despite the liquid cell design, photodecomposition was still observed, with a minimum observable signal plateauing in $\sim 120 \mathrm{~s}$, limiting accumulation times below this value (Figure F.4) 


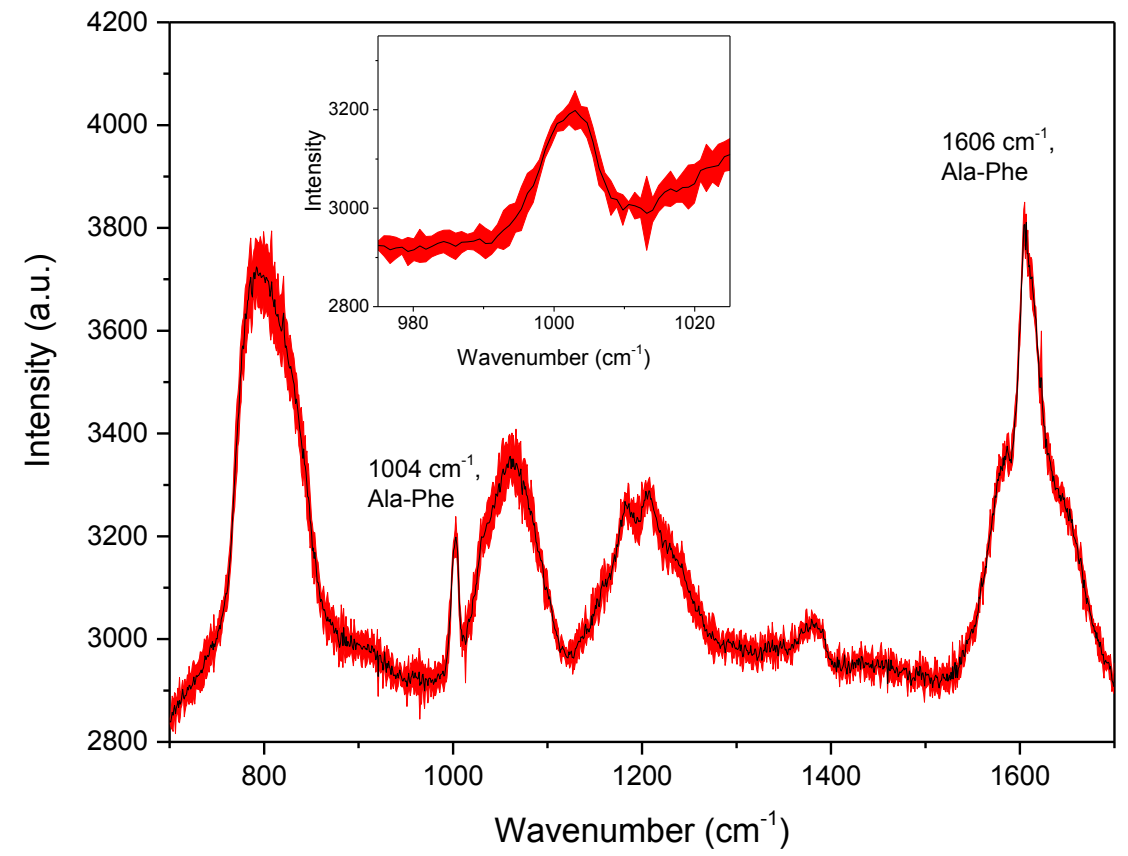

Figure 5.4. UV Raman spectrum of $10 \mathrm{mM}$ Ala-Phe over an InNP film collected at $229 \mathrm{~nm}$. The spectrum shown is the average over 5 spectra accumulated at different locations on the slide. The ring breathing mode at $1004 \mathrm{~cm}^{-1}$ (inset) is used for quantification of the signal as it is most clear from the background. Large features in the background signal at 790,1060 and $1210 \mathrm{~cm}^{-1}$ are from the fused quartz windows and substrate.

\subsubsection{Synthesis of aluminium nanoparticles.}

Aluminium is predicted to be a higher quality plasmonic material in the desired spectral region $(\sim 229 \mathrm{~nm})$ than indium..$^{18,31}$ Aluminium is also a strong candidate as an alternative plasmonic material, as opposed to $\mathrm{Ag}$ or $\mathrm{Au}$, since it is a relatively inexpensive, earth-abundant metal and its plasmon frequency is tunable over a wide range from ultra-violet to the full visible spectrum. ${ }^{47} \mathrm{Al}$ nanoparticles were used in order to test their DUV-SERS response in comparison to In nanoparticles. Controlled synthesis of Al nanoparticles is difficult however, due to the high reactivity of aluminium precursors and the susceptibility of the particles themselves to oxidation. ${ }^{48}$

Aluminum nanoparticles were synthesized by the procedure outlined in section 5.2. developed by Halas et al. ${ }^{33}$ In this method N,N-dimethylethylamine alane is thermally decomposed, catalyzed by titanium (IV) isopropoxide, in 1,4-dioxane and stabilized by oleic acid. The role of dioxane as a coordinating solvent is key in the formation and shape-control of the nanocrystals, the size of which can be controlled by using a mixture of tetrahydrofuran (THF) and dioxane. ${ }^{33,49}$ Like 
with In nanoparticles, exposure to ambient conditions produces a self-limiting oxide layer around the nanocrystals.

The synthesized Al nanoparticles were polyhedral in geometry, with a size of $65 \pm 12 \mathrm{~nm}$. A variety of shapes are produced, their exact geometry is difficult to determine from the transmission electron micrographs and at this size not all particles form well-defined polyhedral. However, the particles are expected to predominately form icosahedra and truncated bipyramids. ${ }^{33}$ A conformal $\mathrm{Al}_{2} \mathrm{O}_{3}$ native oxide shell can be observed (Figure 5.5c) with a thickness of $\sim 2.5 \mathrm{~nm}$. The nanoparticles have a broad UV-Vis extinction spectrum at $316 \mathrm{~nm}$ (Figure 5.5a), with a remarkably similar profile to the InNP shown previously in Figure 5.1.

a
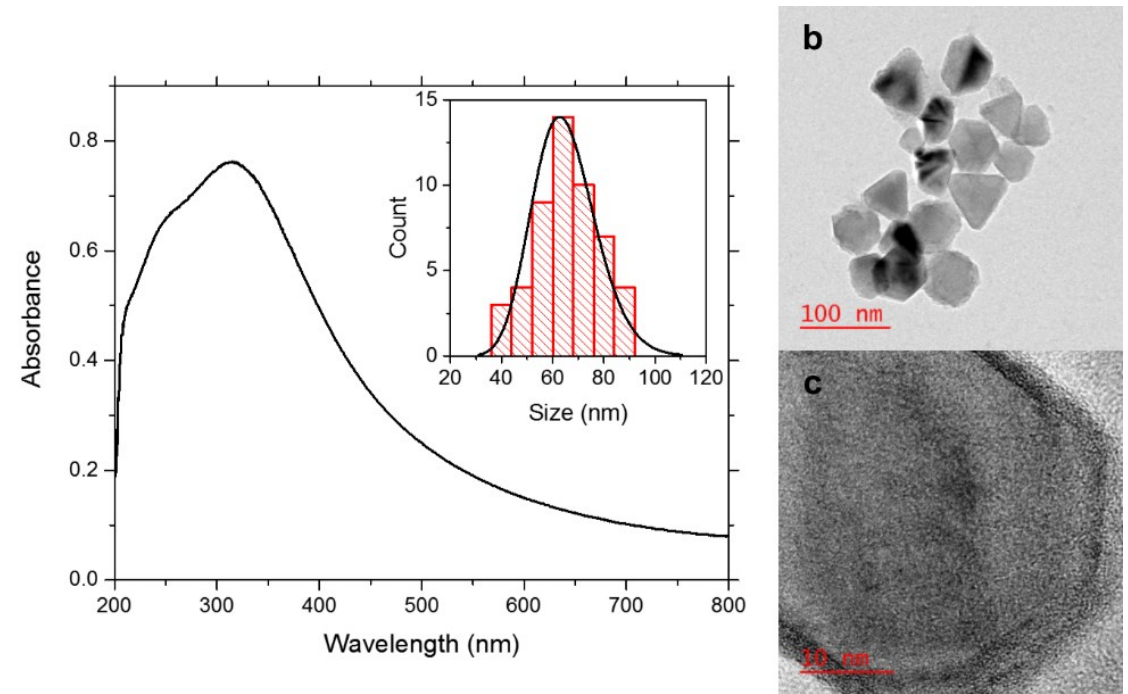

Figure 5.5. a) UV-Vis spectroscopy of AINP suspended in ethanolic solution inset with a histogram of measured sizes $(n=50)$ of the nanocrystals found to be $65 \pm 12 \mathrm{~nm}$ b) a transmission electron micrograph displaying a sample of the synthesized nanocrystals which vary in geometry c) a close-up TEM image displaying a nanocrystal and $\mathrm{a} \sim 2.5 \mathrm{~nm}$ native $\mathrm{Al}_{2} \mathrm{O}_{3}$ shell.

\subsubsection{UV Raman spectroscopy over aluminium nanocrystals}

Similar approaches were used to measure the DUV-SERS response of aluminium nanoparticles as were performed with indium nanoparticles. The strategies employed were: conjugation with PhTMS, thin $\mathrm{SiO}_{2}$ coating followed by conjugation with PhTMS, and Ala-Phe over deposited films. In this case films were deposited by spin-coating rather than the Langmuir-Blodgett technique and yielded rather uniformly distributed particles (Figure F.6).

The DUV-Raman response of AINP in these cases mirrored what was observed for InNP. No Raman response of PhTMS over the AINP was observed. Failure of binding to the $\mathrm{Al}_{2} \mathrm{O}_{3}$ oxide 
surface is not suspected as this functionalization has been demonstrated and confirmed with visible-SERS measurements. ${ }^{41}$ Nevertheless over-coating with thin $\mathrm{SiO}_{2}$ was performed (Figure F.7), though no Raman response was detected. While Ala-Phe was detectable in solution over the AINP films, no statistically significant SERS enhancement was quantified.

\subsubsection{Numerical modelling of indium and aluminium nanoparticles}

To investigate the potential for SERS enhancement for the above experimental conditions and establish a theoretical basis for the observations (or lack thereof), finite-difference time-domain (FDTD) calculations were employed. FDTD is a numerical computational technique which can be used to predict the far-field and near-field response of optical materials, and is a powerful tool for the predictive and explorative analysis of plasmonic nanostructures. ${ }^{50}$

\subsubsection{Indium nanoparticles}

The indium nanoparticles are represented by a $55 \mathrm{~nm}$ diameter particle in a refractive index of $n=1.33$ ( water) with a $3 \mathrm{~nm}$ shell of $n=2.1$ to represent $\ln _{2} \mathrm{O}_{3}$. Even though $60 \mathrm{~nm}$ was the mean experimental size in Figure 5.1, $55 \mathrm{~nm}$ was the mode size, and represented the closer peak position in the model (Figure 5.6a, $324 \mathrm{~nm}$ ). In general, the peak positions were found to be redshifted compared to what was experimentally expected for all sizes of particles. This may indicate the oxide film is not complete or has an effective refractive index below the bulk for $\ln _{2} \mathrm{O}_{3}$. 

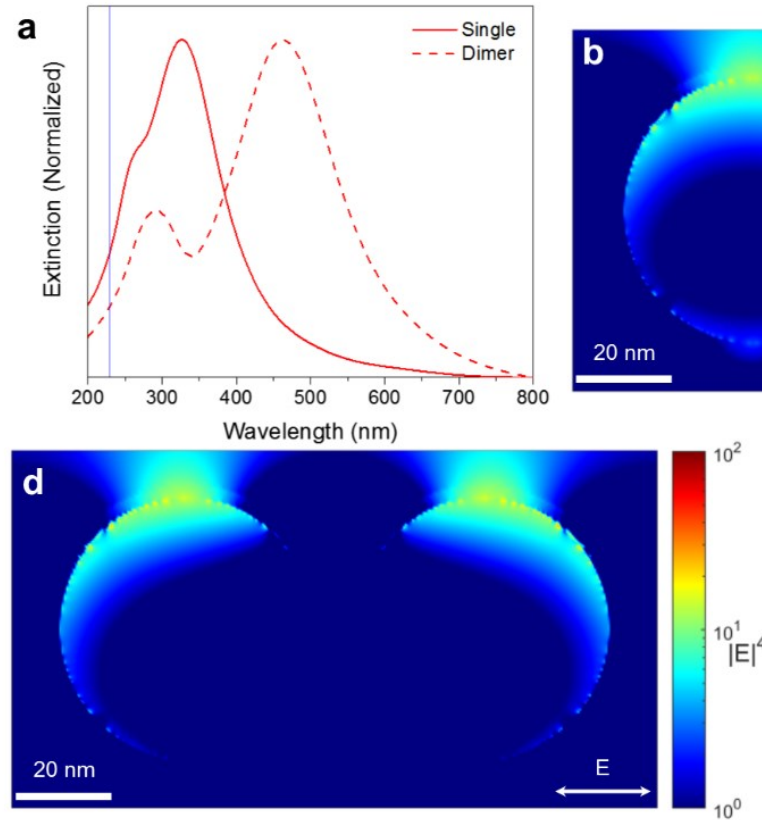
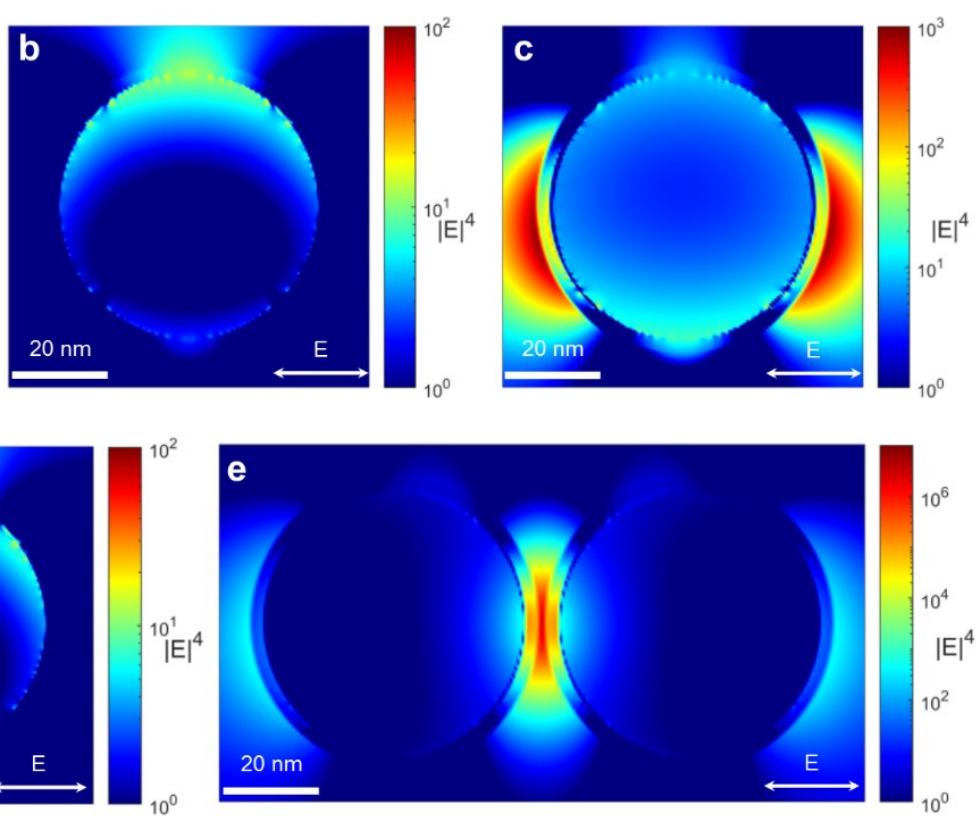

Figure 5.6. a) Calculated extinction cross-sections of $55 \mathrm{~nm}$ InNP with a $3 \mathrm{~nm}$ oxide shell, showing a single, isolated particle, and as well as a dimer of particles with an interparticle spacing of $1 \mathrm{~nm}$ (from the end of the oxide shell). b,c) the calculated SERS electromagnetic enhancement factor $\left(|E|^{4}\right)$ around the nanoparticles at the excitation wavelength $(229 \mathrm{~nm})$ and at their maximum $(324 \mathrm{~nm})$ respectively. d,e) the calculated enhancement of a nanoparticle dimer at $229 \mathrm{~nm}$ and the maximum $(460 \mathrm{~nm})$. Under the experimental conditions the enhancement is predicted to be $<10^{2}$ whereas the potential maximum enhancement factor if a nanoparticle dimer is up to $10^{7}$.

FDTD calculations were also used to predict the electromagnetic Raman enhancement factor using the $|E|^{4}$ approximation. ${ }^{51}$ This approximation is valid assuming there is negligible difference in enhancement between the excitation wavelength and Raman-enhanced band, which in the case of the $\sim 1000 \mathrm{~cm}^{-1}$ band represents a $5 \mathrm{~nm}$ difference. At the experimental excitation wavelength $(229 \mathrm{~nm})$ (Figure 5.6b,d) the observed enhancement is predicted to be all-but negligible with the maximal $|\mathrm{E}|^{4}$ enhancement of $<10^{2}$. This is a stark contrast from the predicted enhancement at the particle's maximum extinction (Figure 5.6c,e): $\sim 10^{3}$ for an individual particle and up to $10^{7}$ in the hotspot of a nanoparticle dimer. The maximal field enhancement is expected to coincide with the maximal particle extinction, ${ }^{31}$ however these maximums are not in the desired deep-UV spectral region, but rather in the near-UV and in the visible for the individual particle and dimer respectively. This indicates that InNP may not be optimal for deep-UV SERS enhancement. However, their strong plasmonic response in the near-UV, may find traction in niche applications such as surface-enhanced fluourescence ${ }^{52}$ and biomolecule sensing. ${ }^{53}$ 


\subsubsection{Aluminium nanoparticles}

The far-field and near-field response of aluminium particles were calculated in the same manner as the indium particles. The model consisted of a $60 \mathrm{~nm}$ aluminium sphere with a $2 \mathrm{~nm}$ $\mathrm{Al}_{2} \mathrm{O}_{3}$ shell $(n=1.80)$ in a background index of $n=1.33$. A sphere was used for simplicity despite the known geometry of the particles to be polyhedral. This approximation is valid, at least for the predicted icosahedral geometry of the particles, ${ }^{33}$ and necessary as the transmission electron micrographs in Figure 5.5 provide limited insight into the 3D geometry of the particles. The modelled particles display a central peak position of $306 \mathrm{~nm}$ (Figure 5.7), but curiously also display a strong quadrupolar peak at $224 \mathrm{~nm} .{ }^{33}$ This peak is not clearly visible in the experimental spectra, but rather a weaker shoulder at $250 \mathrm{~nm}$ is observed in Figure 5.5. It is believed the size and shape polydispersity of the ensemble measurement reduce the apparent intensity of this peak, as well as a greater degree of shape-dependence at this nanoparticle's scale; the spherical approximation may not be valid for smaller particles.

a

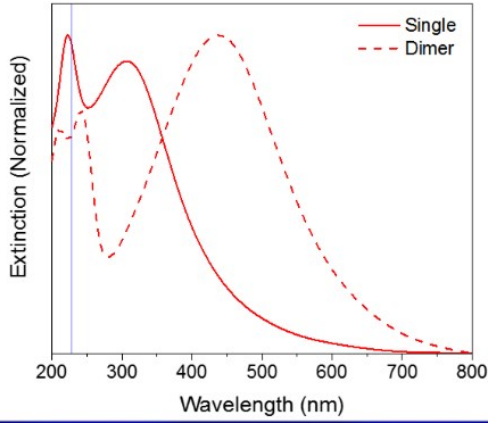

d

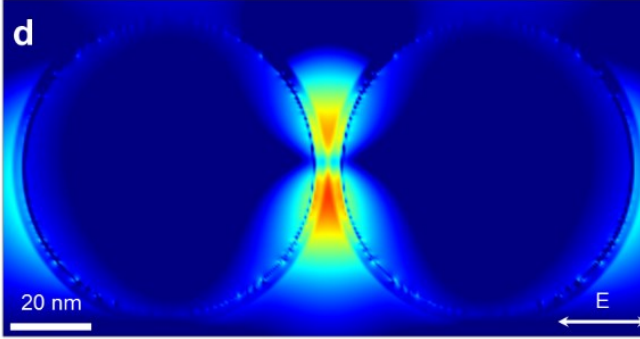

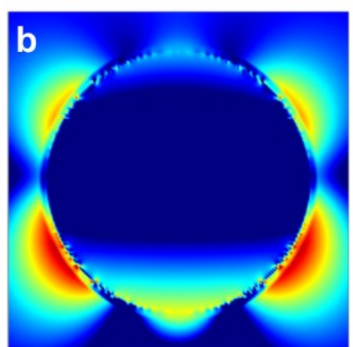
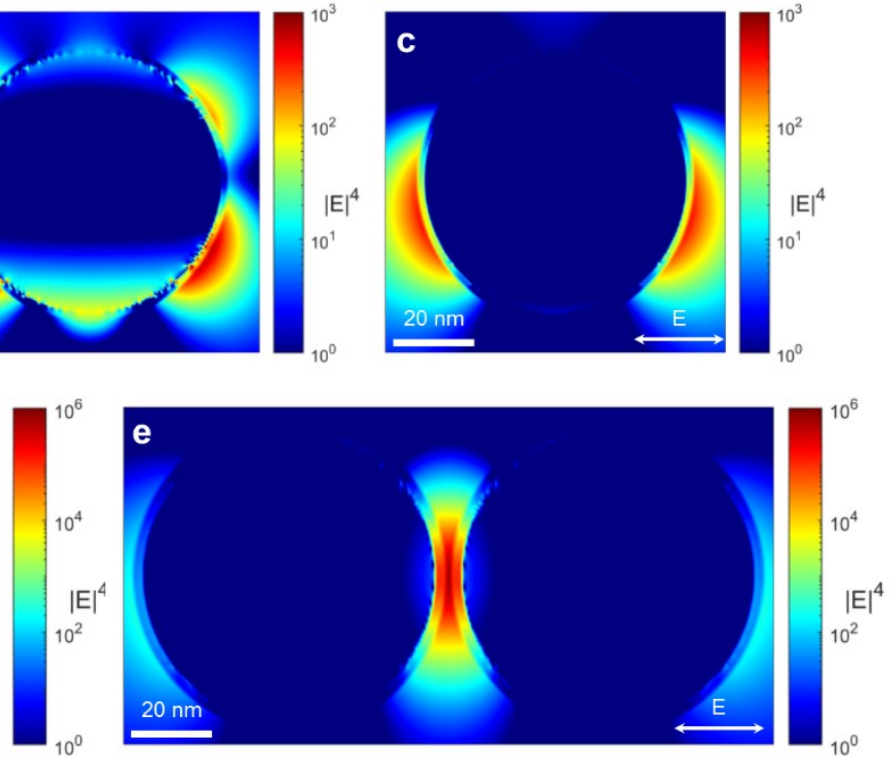

Figure 5.7. a) Calculated extinction cross-sections of $60 \mathrm{~nm}$ AINP with a $2 \mathrm{~nm}$ oxide shell, showing a single, isolated particle, and as well as a dimer of particles with an interparticle spacing of $1 \mathrm{~nm}$ (from the end of the oxide shell). b,c) the calculated SERS electromagnetic enhancement factor $\left(|\mathrm{E}|^{4}\right)$ around the nanoparticles at the excitation wavelength $(229 \mathrm{~nm})$ and at their maximum $(306 \mathrm{~nm})$ respectively. d,e) the calculated enhancement of a nanoparticle dimer at $229 \mathrm{~nm}$ and the maximum $(440 \mathrm{~nm})$. Unlike the indium particles, AINP display almost equally strong enhancement at both positions due to the strong quadrupolar mode. 
Unlike in the indium nanoparticles, the SERS enhancement at $229 \mathrm{~nm}$ and the predicted maximum are comparable. Individual particles display an $|E|^{4}$ enhancement of $\sim 10^{3}$ while the dimers display a maximum enhancement factor of $\sim 10^{6}$ (Figure $5.7 \mathrm{~b}-\mathrm{e}$ ). This is due to the strong quadrupolar mode observed in the model, which does not shift far in the case of the dimer. While this bodes well for the potential of AINP for DUV-SERS it should be noted once again a quadrupolar mode of this magnitude is not apparent in the ensemble measurements; the Q mode is only weakly discernable(Figure 5.5). Thus, the potential experimental enhancement is likely not to the same magnitude as predicted by these models. However, these models do demonstrate the potential for AINP in SERS. Recent improvements in the size and shape selectivity in the synthesis of aluminium nanocrystals, ${ }^{49,54}$ in conjunction with approaches which involve plasmon-mode hybridization by introducing anisotropy may serve to enhance this quadrupolar mode. ${ }^{55}$ As such there still exists strong potential for aluminium nanocrystals to be used for DUV-SERS and other DUV plasmonic applications.

\subsection{Conclusion and Future Outlook}

The synthesis of indium and aluminium nanocrystals displaying a UV-plasmonic response was achieved, however investigations into their DUV-SERS response did not yield appreciable SERS enhancement. Concerning indium nanoparticles as a DUV-SERS material, the results of this work are consistent with other observations which report low SERS enhancement, ${ }^{56}$ and predictions by FDTD. Indium may be a more suitable material for SERS performed at near-UV, or visible wavelengths. ${ }^{27,57}$

Aluminium nanocrystals on the other hand have significantly more potential for DUV-SERS. While DUV-SERS enhancement was not observed in this work, numerous other works exist displaying SERS-enhancement factors up to $\sim 10^{6}$ on Al-based substrates. ${ }^{6,24-26}$ These examples do not use colloidal AINPs but rather a combination of lithographic techniques and film-deposition. A strong near-IR SERS response has been demonstrated with similar colloidal aluminium nanocrystals. ${ }^{41}$ The FDTD calculations in this work suggest potential for AINPs in DUV-SERS applications, especially if leveraging recent advances in size and shape-controlled synthesis to 
promote the intensity of the quadrupolar mode. ${ }^{49,54}$ Aluminium nanocrystals may also see further use in other applications; the highly tunable plasmonic response of aluminium nanostructures makes them well suited to plasmonic-colour patterning, ${ }^{58,59}$ and as a highly earth-abundant material is attractive for plasmonic photocatalysis. ${ }^{60}$

\subsection{References}

(1) Atwater, H. a; Polman, A. Plasmonics for Improved Photovoltaic Devices. Nat. Mater. 2010, 9 (3), 205-213.

(2) Knight, M. W.; Sobhani, H.; Nordlander, P.; Halas, N. J. Photodetection with Active Optical Antennas. Science (80-. ). 2011, 332 (6030), 702-704.

(3) Brongersma, M. L.; Halas, N. J.; Nordlander, P. Plasmon-Induced Hot Carrier Science and Technology. Nat. Nanotechnol. 2015, 10 (1), 25-34.

(4) Willets, K. a; Van Duyne, R. P. Localized Surface Plasmon Resonance Spectroscopy and Sensing. Annu. Rev. Phys. Chem. 2007, 58, 267-297.

(5) Yang, H. U.; D’Archangel, J.; Sundheimer, M. L.; Tucker, E.; Boreman, G. D.; Raschke, M. B. Optical Dielectric Function of Silver. Phys. Rev. B 2015, 91 (23), 235137.

(6) Sigle, D. O.; Perkins, E.; Baumberg, J. J.; Mahajan, S. Reproducible Deep-UV SERRS on Aluminum Nanovoids. J. Phys. Chem. Lett. 2013, 4 (9), 1449-1452.

(7) Bisio, F.; Proietti Zaccaria, R.; Moroni, R.; Maidecchi, G.; Alabastri, A.; Gonella, G.; Giglia, A.; Andolfi, L.; Nannarone, S.; Mattera, L.; Canepa, M. Pushing the High-Energy Limit of Plasmonics. ACS Nano 2014, 8 (9), 9239-9247.

(8) Stiles, P. L.; Dieringer, J. a; Shah, N. C.; Van Duyne, R. P. Surface-Enhanced Raman Spectroscopy. Annu. Rev. Anal. Chem. (Palo Alto. Calif). 2008, 1, 601-626.

(9) Kneipp, K.; Wang, Y.; Kneipp, H.; Perelman, L. T.; Itzkan, I.; Dasari, R. R.; Feld, M. S. Single Molecule Detection Using Surface-Enhanced Raman Scattering ( SERS ). Phys. Rev. Lett. 1997, 78 (9), 1667-1670.

(10) Nie, S. Emory, S. Probing Single Molecules and Single Nanoparticles by SurfaceEnhanced Raman Scattering. Science (80-. ). 1997, 275 (5303), 1102-1106.

(11) Jeanmaire, D. L.; Van Duyne, R. P. Surface Raman Spectroelectrochemistry. J. Electroanal. Chem. Interfacial Electrochem. 1977, 84 (1), 1-20.

(12) Luo, S. C.; Sivashanmugan, K.; Liao, J. Der; Yao, C. K.; Peng, H. C. Nanofabricated SERS-Active Substrates for Single-Molecule to Virus Detection in Vitro: A Review. Biosens. Bioelectron. 2014, 61, 232-240.

(13) Xu, M. L.; Gao, Y.; Han, X. X.; Zhao, B. Detection of Pesticide Residues in Food Using Surface-Enhanced Raman Spectroscopy: A Review. J. Agric. Food Chem. 2017, 65 (32), 6719-6726.

(14) Fikiet, M. A.; Khandasammy, S. R.; Mistek, E.; Ahmed, Y.; Halámková, L.; Bueno, J.; Lednev, I. K. Surface Enhanced Raman Spectroscopy: A Review of Recent Applications in Forensic Science. Spectrochim. Acta - Part A Mol. Biomol. Spectrosc. 2018, 197, 255260.

(15) Wabuyele, M. B.; Yan, F.; Griffin, G. D.; Vo-Dinh, T. Hyperspectral Surface-Enhanced Raman Imaging of Labeled Silver Nanoparticles in Single Cells. Rev. Sci. Instrum. 2005, 
$76(6)$.

(16) Kumar, N.; Mignuzzi, S.; Su, W.; Roy, D. Tip-Enhanced Raman Spectroscopy : Principles and Applications. EPJ Tech. Instrum. 2015.

(17) Restaino, S. M.; White, I. M. A Critical Review of Flexible and Porous SERS Sensors for Analytical Chemistry at the Point-of-Sample. Anal. Chim. Acta 2019, 1060, 17-29.

(18) Blaber, M. G.; Arnold, M. D.; Ford, M. J. A Review of the Optical Properties of Alloys and Intermetallics for Plasmonics. J. Phys. Condens. Matter 2010, 22 (14), 143201.

(19) Efremov, E. V.; Ariese, F.; Gooijer, C. Achievements in Resonance Raman Spectroscopy. Review of a Technique with a Distinct Analytical Chemistry Potential. Anal. Chim. Acta 2008, 606 (2), 119-134.

(20) Oladepo, S. A.; Xiong, K.; Hong, Z.; Asher, S. A.; Handen, J.; Lednev, I. K. UV Resonance Raman Investigations of Peptide and Protein Structure and Dynamics. Chem. Rev. 2012, 112 (5), 2604-2628.

(21) Gares, K. L.; Hufziger, K. T.; Bykov, S. V.; Asher, S. A. Review of Explosive Detection Methodologies and the Emergence of Standoff Deep UV Resonance Raman. J. Raman Spectrosc. 2016, 47 (1), 124-141.

(22) Jakubek, R. S.; Handen, J.; White, S. E.; Asher, S. A.; Lednev, I. K. Ultraviolet Resonance Raman Spectroscopic Markers for Protein Structure and Dynamics. TrAC - Trends Anal. Chem. 2018, 103, 223-229.

(23) McMahon, J. M.; Schatz, G. C.; Gray, S. K. Plasmonics in the Ultraviolet with the Poor Metals Al, Ga, In, Sn, TI, Pb, and Bi. Phys. Chem. Chem. Phys. 2013, 15 (15), 54155423.

(24) Ding, T.; Sigle, D. O.; Herrmann, L. O.; Wolverson, D.; Baumberg, J. J. Nanoimprint Lithography of Al Nanovoids for Deep-UV SERS. ACS Appl. Mater. Interfaces 2014, 6 (20), 17358-17363.

(25) Sharma, B.; Cardinal, M. F.; Ross, M. B.; Zrimsek, A. B.; Bykov, S. V.; Punihaole, D.; Asher, S. A.; Schatz, G. C.; Van Duyne, R. P. Aluminum Film-Over-Nanosphere Substrates for Deep-UV Surface-Enhanced Resonance Raman Spectroscopy. Nano Lett. 2016, 16 (12), 7968-7973.

(26) Jha, S. K.; Ahmed, Z.; Agio, M.; Ekinci, Y.; Löffler, J. F. Deep-UV Surface-Enhanced Resonance Raman Scattering of Adenine on Aluminum Nanoparticle Arrays. J. Am. Chem. Soc. 2012, 134 (4), 1966-1969.

(27) Das, R.; Soni, R. K. Synthesis and Surface-Enhanced Raman Scattering of Indium Nanotriangles and Nanowires. RSC Adv. 2017, 7 (51), 32255-32263.

(28) Yang, Y.; Callahan, J. M.; Kim, T. H.; Brown, A. S.; Everitt, H. O. Ultraviolet Nanoplasmonics: A Demonstration of Surface-Enhanced Raman Spectroscopy, Fluorescence, and Photodegradation Using Gallium Nanoparticles. Nano Lett. 2013, 13 (6), 2837-2841.

(29) Watson, A. M.; Zhang, X.; Alcaraz de la Osa, R.; Marcos Sanz, J.; González, F.; Moreno, F.; Finkelstein, G.; Liu, J.; Everitt, H. O. Rhodium Nanoparticles for Ultraviolet Plasmonics. Nano Lett. 2015, 15 (2), 1095-1100.

(30) Ponzellini, P.; Giovannini, G.; Cattarin, S.; Zaccaria, R. P.; Marras, S.; Prato, M.; Schirato, A.; D’Amico, F.; Calandrini, E.; De Angelis, F.; Yang, W.; Jin, H.-J.; Alabastri, A.; Garoli, D. Metallic Nanoporous Aluminum-Magnesium Alloy for UV-Enhanced Spectroscopy. J. Phys. Chem. C 2019, 123 (33), 20287-20296.

(31) Ross, M. B.; Schatz, G. C. Aluminum and Indium Plasmonic Nanoantennas in the Ultraviolet. J. Phys. Chem. C 2014, 118 (23), 12506-12514. 
(32) Kind, C.; Feldmann, C. One-Pot Synthesis of In 0 Nanoparticles with Tuned Particle Size and High Oxidation Stability. 2011, 55 (7), 7-9.

(33) McClain, M. J.; Schlather, A. E.; Ringe, E.; King, N. S.; Liu, L.; Manjavacas, A.; Knight, M. W.; Kumar, I.; Whitmire, K. H.; Everitt, H. O.; Nordlander, P.; Halas, N. J. Aluminum Nanocrystals. Nano Lett. 2015, 15 (4), 2751-2755.

(34) Magnan, F.; Gagnon, J.; Fontaine, F.-G.; Boudreau, D. Indium@silica Core-Shell Nanoparticles as Plasmonic Enhancers of Molecular Luminescence in the UV Region. Chem. Commun. 2013, 49 (81), 9299.

(35) Xia, Y.; Xiong, Y.; Lim, B.; Skrabalak, S. E. Shape-Controlled Synthesis of Metal Nanocrystals: Simple Chemistry Meets Complex Physics? Angew. Chemie Int. Ed. 2009, 48 (1), 60-103.

(36) Herring, C. Some Theorems on the Free Energies of Crystal Surfaces. Phys. Rev. 1951, $82(1), 87-93$.

(37) Sun, J.; Simon, S. L. The Melting Behavior of Aluminum Nanoparticles. Thermochim. Acta 2007, 463 (1-2), 32-40.

(38) Coombes, C. J. The Melting of Small Particles of Lead and Indium. J. Phys. F Met. Phys. 1972, 2 (3), 441-449.

(39) Xie, D.; Wang, M. P.; Qi, W. H.; Cao, L. F. Thermal Stability of Indium Nanocrystals: A Theoretical Study. Mater. Chem. Phys. 2006, 96 (2-3), 418-421.

(40) Sutter, E.; Sutter, P. Size-Dependent Room Temperature Oxidation of In Nanoparticles. J. Phys. Chem. C 2012, 116 (38), 20574-20578.

(41) Tian, S.; Neumann, O.; McClain, M. J.; Yang, X.; Zhou, L.; Zhang, C.; Nordlander, P.; Halas, N. J. Aluminum Nanocrystals: A Sustainable Substrate for Quantitative SERSBased DNA Detection. Nano Lett. 2017, 17 (8), 5071-5077.

(42) Wasserman, S. R.; Tao, Y. T.; Whitesides, G. M. Structure and Reactivity of Alkylsiloxane Monolayers Formed by Reaction of Alkyltrichlorosilanes on Silicon Substrates. Langmuir 1989, 5 (4), 1074-1087.

(43) Stöber, W.; Fink, A.; Bohn, E. Controlled Growth of Monodisperse Silica Spheres in the Micron Size Range. J. Colloid Interface Sci. 1968, 26 (1), 62-69.

(44) Mulder, C. A. M.; Damen, A. A. J. M. Raman Analysis of the Initial Stages of the Hydrolysis and Polymerization of Tetraethylorthosilicate. J. Non. Cryst. Solids 1987, 93 (1), 169-178.

(45) Li, Y.-S.; Wang, Y.; Ceesay, S. Vibrational Spectra of Phenyltriethoxysilane, Phenyltrimethoxysilane and Their Sol-Gels. Spectrochim. Acta. A. Mol. Biomol. Spectrosc. 2009, 71 (5), 1819-1824.

(46) Blackie, E. J.; Le Ru, E. C.; Etchegoin, P. G. Single-Molecule Surface-Enhanced Raman Spectroscopy of Nonresonant Molecules. J. Am. Chem. Soc. 2009, 131 (40), 1446614472.

(47) Knight, M. W.; King, N. S.; Liu, L.; Everitt, H. O.; Nordlander, P.; Halas, N. J. Aluminum for Plasmonics. ACS Nano 2014, 8 (1), 834-840.

(48) Meziani, M. J.; Bunker, C. E.; Lu, F.; Li, H.; Wang, W.; Guliants, E. a; Quinn, R. a; Sun, Y.P. Formation and Properties of Stabilized Aluminum Nanoparticles. ACS Appl. Mater. Interfaces 2009, 1 (3), 703-709.

(49) Clark, B. D.; Desantis, C. J.; Wu, G.; Renard, D.; McClain, M. J.; Bursi, L.; Tsai, A. L.; Nordlander, P.; Halas, N. J. Ligand-Dependent Colloidal Stability Controls the Growth of Aluminum Nanocrystals. J. Am. Chem. Soc. 2019, 141 (4), 1716-1724. 
(50) Gray, S. K. Theory and Modeling of Plasmonic Structures. J. Phys. Chem. C 2013, 117 (5), 1983-1994.

(51) Stiles, P. L.; Dieringer, J. A.; Shah, N. C.; Van Duyne, R. P. Surface-Enhanced Raman Spectroscopy. Annu. Rev. Anal. Chem. 2008, 1 (1), 601-626.

(52) Magnan, F.; Gagnon, J.; Fontaine, F. G.; Boudreau, D. Indium@silica Core-Shell Nanoparticles as Plasmonic Enhancers of Molecular Luminescence in the UV Region. Chem. Commun. 2013, 49 (81), 9299-9301.

(53) Marín, A. G.; García-Mendiola, T.; Bernabeu, C. N.; Hernández, M. J.; Piqueras, J.; Pau, J. L.; Pariente, F.; Lorenzo, E. Gallium Plasmonic Nanoparticles for Label-Free DNA and Single Nucleotide Polymorphism Sensing. Nanoscale 2016, 8 (18), 9842-9851.

(54) Clark, B. D.; Jacobson, C. R.; Lou, M.; Renard, D.; Wu, G.; Bursi, L.; Ali, A. S.; Swearer, D. F.; Tsai, A.-L.; Nordlander, P.; Halas, N. J. Aluminum Nanocubes Have Sharp Corners. ACS Nano 2019, 13, acsnano.9b05277.

(55) Zhang, S.; Bao, K.; Halas, N. J.; Xu, H.; Nordlander, P. Substrate-Induced Fano Resonances of a Plasmonic Nanocube: A Route to Increased-Sensitivity Localized Surface Plasmon Resonance Sensors Revealed. Nano Lett. 2011, 11 (4), 1657-1663.

(56) Kumamoto, Y.; Taguchi, A.; Honda, M.; Watanabe, K.; Saito, Y.; Kawata, S. Indium for Deep-Ultraviolet Surface-Enhanced Resonance Raman Scattering. ACS Photonics 2014, 1 (7), 598-603.

(57) Das, R.; Soni, R. K. Highly Stable In@SiO2 Core-Shell Nanostructures for Ultraviolet Surface-Enhanced Raman Spectroscopy. Appl. Surf. Sci. 2019, 489 (May), 755-765.

(58) Ho, J.; Dong, Z.; Daqiqeh Rezaei, S.; Ng, R. J. H.; H. H. Koay, E.; Ramakrishna, S.; Yang, J. K. W. Wide-Gamut Plasmonic Color Palettes with Constant Subwavelength Resolution. ACS Nano 2019.

(59) Zhu, X.; Vannahme, C.; Højlund-Nielsen, E.; Mortensen, N. A.; Kristensen, A. Plasmonic Colour Laser Printing. Nat. Nanotechnol. 2016, 11 (4), 325-329.

(60) Zhou, L.; Zhang, C.; McClain, M. J.; Manjavacas, A.; Krauter, C. M.; Tian, S.; Berg, F.; Everitt, H. O.; Carter, E. A.; Nordlander, P.; Halas, N. J. Aluminum Nanocrystals as a Plasmonic Photocatalyst for Hydrogen Dissociation. Nano Lett. 2016, 16 (2), 1478-1484. 


\section{Concluding Remarks}

This work explores the properties and uses of hybrid plasmonic modes in a number of systems. The ability to control the spectral and spatial response of a plasmonic system by introducing asymmetric environments which enable mode-mixing has been demonstrated both theoretically and experimentally. Using such systems to tailor the plasmonic response towards specific applications such as sensing, patterning, and photocatalysis will benefit the field. This work demonstrated several such systems which were improved as a result of using hybridized modes which produced increased sensitivity of LSPR shifts and their spatial resolution.

The use of alternative plasmonic materials also represents a direction which would increase the applicability of the field. This work in large part uses silver nanostructures. While displaying a strong plasmonic response, silver is prone to oxidation which hampers its long-term applicability. Gold is limited in its plasmonic response in the visible region and is prohibitively expensive for large-scale applications.

A promising alternative is aluminium. While the UV response of aluminium is investigated in this work, it is capable of supporting plasmons in the visible range. Recent advances in the synthesis of colloidal AINP enable the potential study of hybrid plasmon modes in this material. ${ }^{1}$ For example, an alternative system which could improve the colour gamut of the system presented in Chapter 5 would be to use aluminium nanocubes (AINC's). Well-controlled AINC's have recently been synthesized in the literature, and thus a system similar to the one produced in this work incorporating AINC's could be produced. Due to the natural resonance frequency of AINC's in the UV, the gap-plasmon could potentially be tuned across the entire visible spectrum, with little interference in the blue-end of the spectrum. Aluminum-based systems have been demonstrated to be among the best for plasmon-generated colours, ${ }^{2-4}$ and is an attractive alternative plasmonic material as it is the most earth-abundant metal and orders of magnitude less expensive than Ag or Au. Aluminum can also be potentially useful in other applications, such as photocatalysis. ${ }^{5}$

Photocatalysis in particular is a burgeoning field of interest in plasmonics due to the recent insight into "hot carriers". ${ }^{6}$ Plasmonic material have been demonstrated to act as photosensitizers to less optically-active photocatalytic materials and show promise in enhancing their activity. ${ }^{7}$ The 
application of these systems rely on efficient light absorption, and the facilitation of efficient surface chemistry. The use of hybridized plasmons for the precise manipulation of their optical properties may be key in providing a route towards realizing efficient plasmon-mediated catalysis.

Plasmonic materials remain a unique avenue for light-matter interactions. There may be yet unexamined uses for plasmonics; these materials may yet find their niche in wide-spread use. A key to their applicability in any case will be the understanding and manipulation of their unique properties with precision and ingenuity.

\section{References}

(1) Clark, B. D.; Jacobson, C. R.; Lou, M.; Renard, D.; Wu, G.; Bursi, L.; Ali, A. S.; Swearer, D. F.; Tsai, A.-L.; Nordlander, P.; Halas, N. J. Aluminum Nanocubes Have Sharp Corners. ACS Nano 2019, 13, acsnano.9b05277.

(2) Tan, S. J.; Zhang, L.; Zhu, D.; Goh, X. M.; Wang, Y. M.; Kumar, K.; Qiu, C.; Yang, J. K. W. Plasmonic Color Palettes for Photorealistic Printing with Aluminum Nanostructures. Nano Lett. 2014, 14 (7), 4023-4029.

(3) Xue, J.; Zhou, Z.-K.; Wei, Z.; Su, R.; Lai, J.; Li, J.; Li, C.; Zhang, T.; Wang, X.-H. Scalable, Full-Colour and Controllable Chromotropic Plasmonic Printing. Nat. Commun. 2015, 6 (May), 8906.

(4) Kristensen, A.; Yang, J. K. W.; Bozhevolnyi, S. I.; Link, S.; Nordlander, P.; Halas, N. J.; Mortensen, N. A. Plasmonic Colour Generation. Nat. Rev. Mater. 2016, 2 (1), 16088.

(5) Robatjazi, H.; Zhao, H.; Swearer, D. F.; Hogan, N. J.; Zhou, L.; Alabastri, A.; McClain, M. J.; Nordlander, P.; Halas, N. J. Plasmon-Induced Selective Carbon Dioxide Conversion on Earth-Abundant Aluminum-Cuprous Oxide Antenna-Reactor Nanoparticles. Nat. Commun. 2017, 8 (1), 1-9.

(6) Brongersma, M. L.; Halas, N. J.; Nordlander, P. Plasmon-Induced Hot Carrier Science and Technology. Nat. Nanotechnol. 2015, 10 (1), 25-34.

(7) Wu, N. Plasmonic Metal-Semiconductor Photocatalysts and Photoelectrochemical Cells: A Review. Nanoscale 2018, 10 (6), 2679-2696. 


\section{Appendices}

\section{Appendix A Supplementary Materials for Chapter 2A}

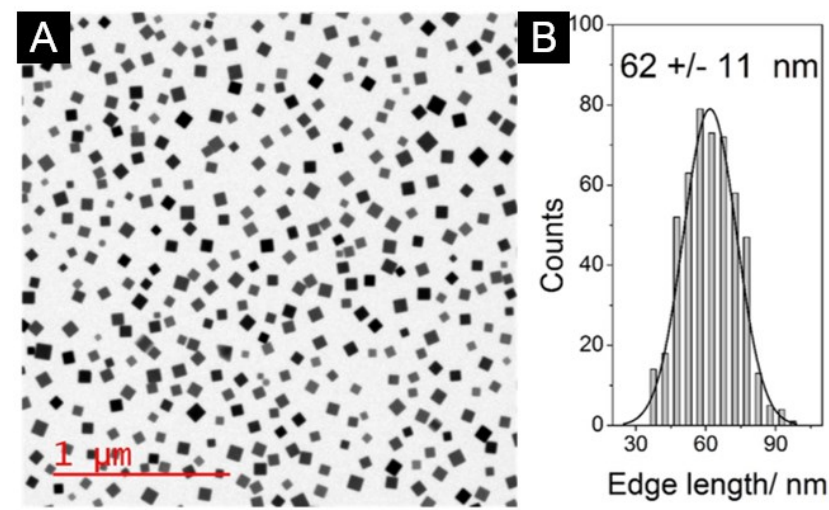

A.1 A) Transmission electron micrograph of AgNC's showing B) a histogram of their measured edge lengths

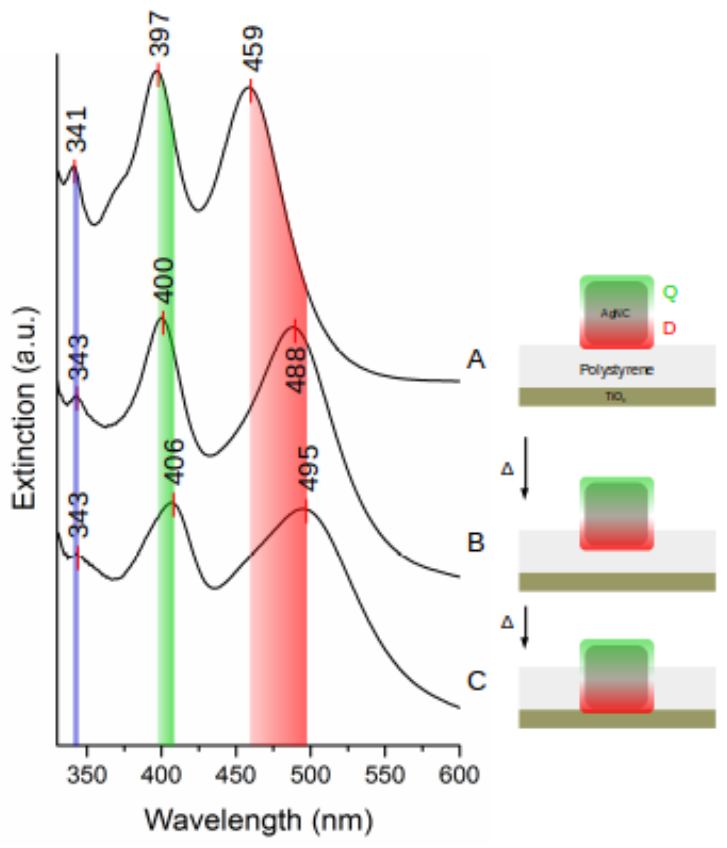

A.2 Experimental schematic: A monolayer of silver nanocubes deposited via the LangmuirSchaeffer technique onto a thin $50 \mathrm{~nm}$ film of polystyrene on a $20 \mathrm{~nm}$ thin film of high refractive index titanium oxide. As the system is heated near the glass transition temperature of the underlying polymer the nanocubes begin to diffuse into the polymer layer. The position of the nanocubes and corresponding extinction spectrum are shown for the initial $(A)$, intermediate $(B)$, and final $(C)$ position. Due to the high refractive index of the underlying titanium oxide, the $D$ mode shifts further when compared to a polystyrene on glass system. The $Q$ mode only shifts marginally as the cubes themselves are larger than the polymer layer and a large portion of the cube remains exposed keeping the $Q$ mode sensitive to the surrounding environment (air) above the composite material 


\section{Appendix B Supplementary Materials for Chapter 2B}

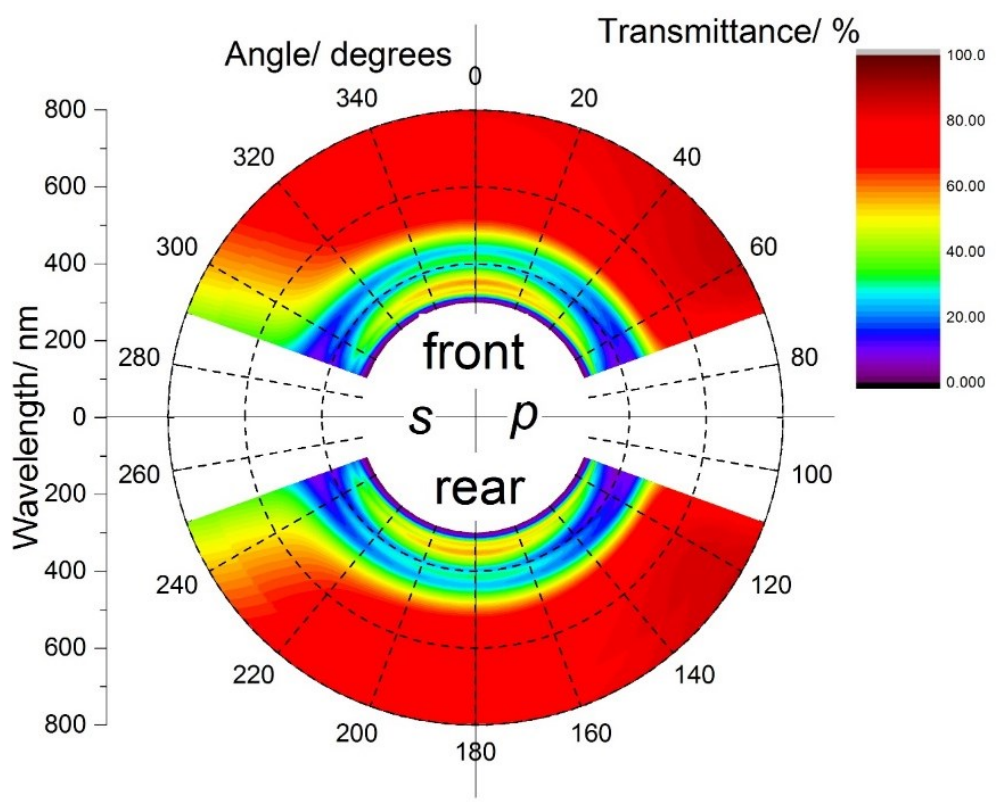

B.1 Transmittance spectral map for uncoated silver nanocubes on a glass substrate. The spectral maps are obtained for front (top) and rear (bottom) illumination geometry, for s (left) and $p$ (right) polarized light.

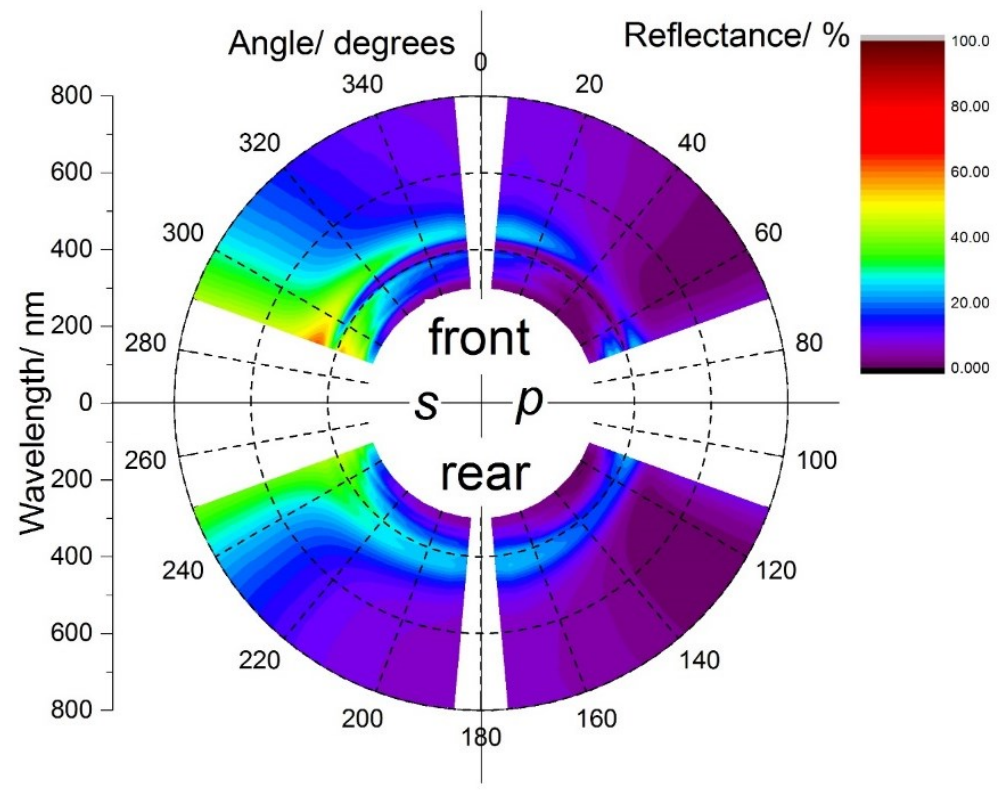

B.2 Reflectance spectral map for uncoated silver nanocubes on a glass substrate. The spectral maps are obtained for front (top) and rear (bottom) illumination geometry, for $s$ (left) and $p$ (right) polarized light. 


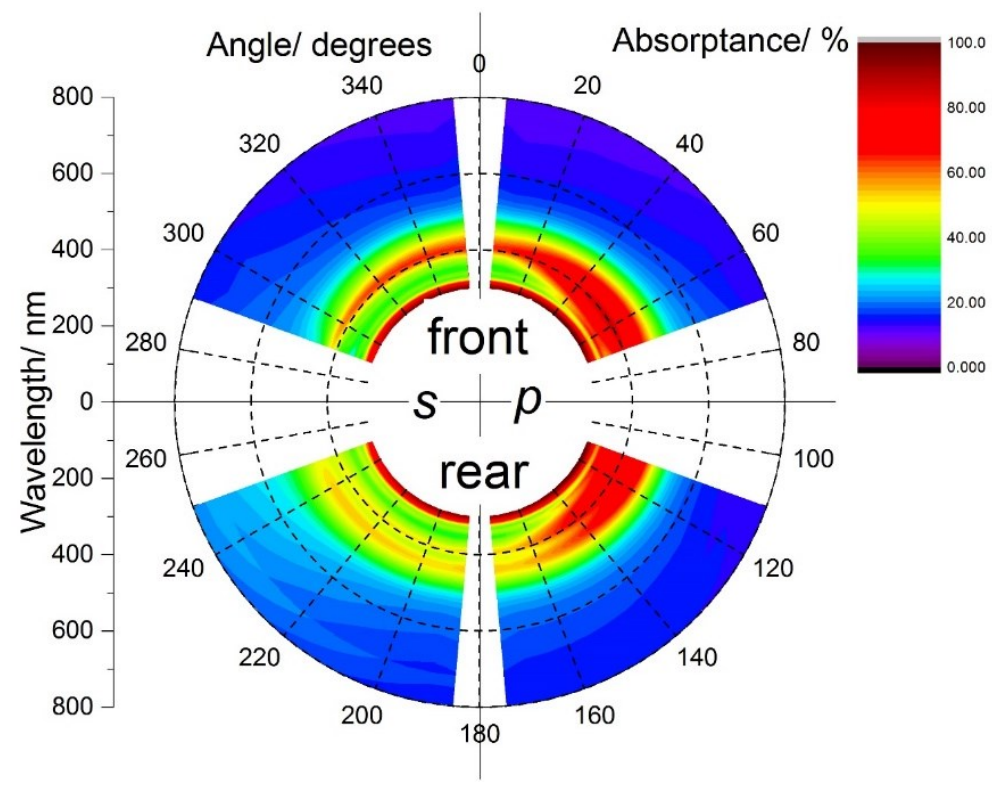

B.3 Absorptance spectral map for uncoated silver nanocubes on a glass substrate, calculated as $100 \%$ less transmittance and reflectance. The spectral maps are obtained for front (top) and rear (bottom) illumination geometry, for $s$ (left) and $p$ (right) polarized light.

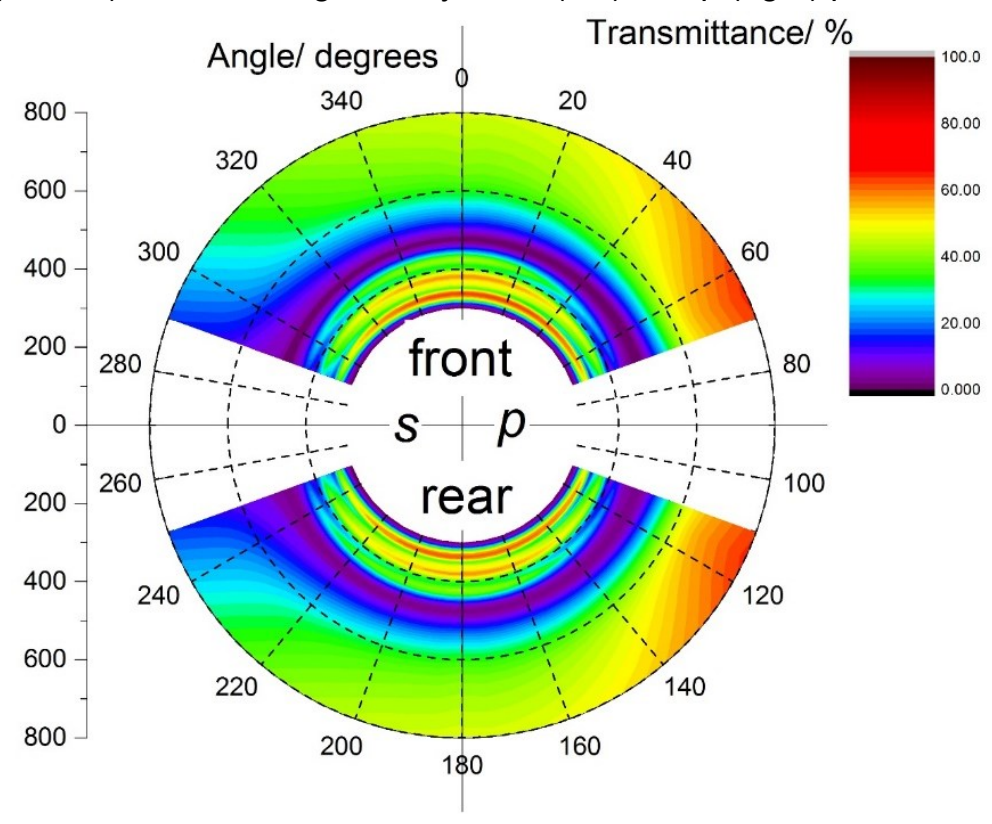

B.4 Transmittance spectral map for silver nanocubes on a glass substrate overcoated with a thin film of alumina and exhibiting sharp LSPR. The spectral maps are obtained for front (top) and rear (bottom) illumination geometry, for $\mathrm{s}$ (left) and $\mathrm{p}$ (right) polarized light. 


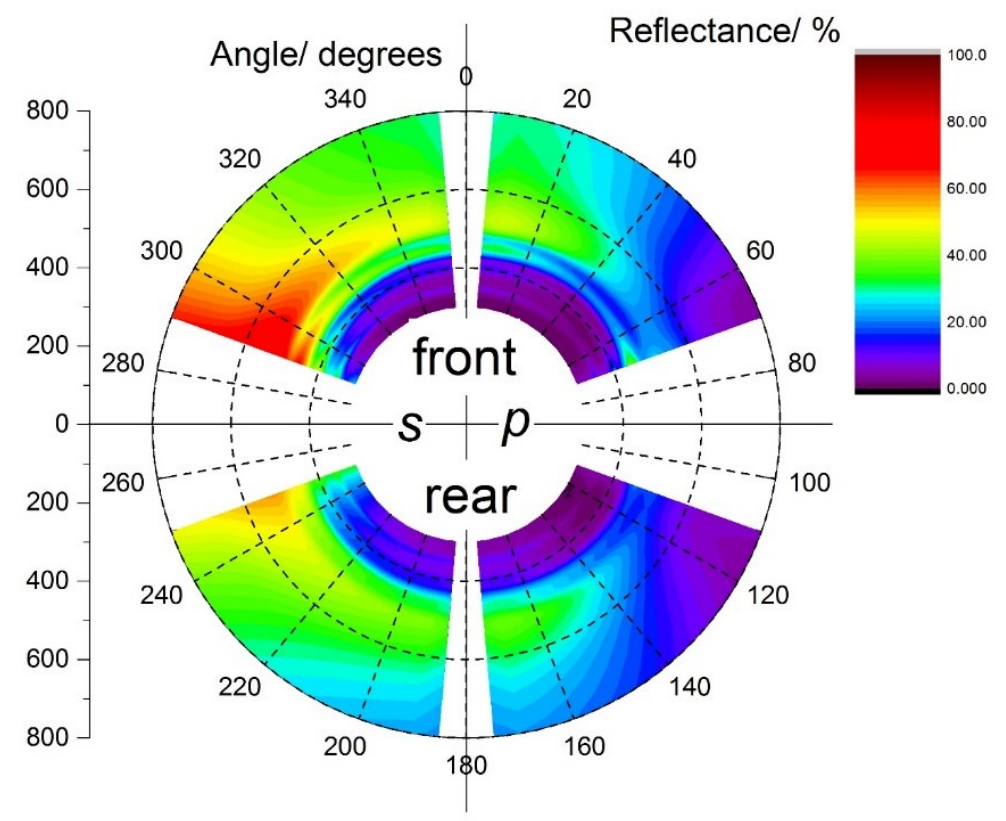

B.5 Reflectance spectral map for silver nanocubes on a glass substrate overcoated with a thin film of alumina and exhibiting sharp LSPR. The spectral maps are obtained for front (top) and rear (bottom) illumination geometry, for $s$ (left) and $\mathrm{p}$ (right) polarized light.

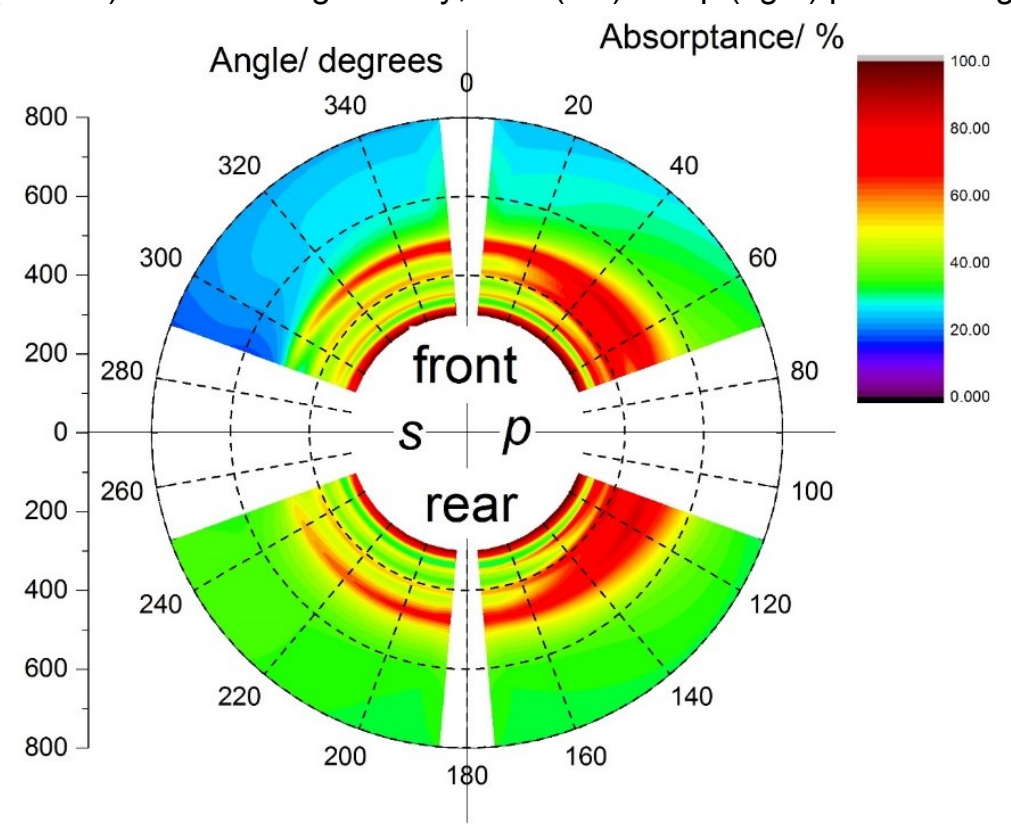

B.6 Absorptance spectral map for silver nanocubes on a glass substrate overcoated with a thin film of alumina and exhibiting sharp LSPR, calculated as $100 \%$ less transmittance and reflectance. The spectral maps are obtained for front (top) and rear (bottom) illumination geometry, for $\mathrm{s}$ (left) and $\mathrm{p}$ (right) polarized light. 


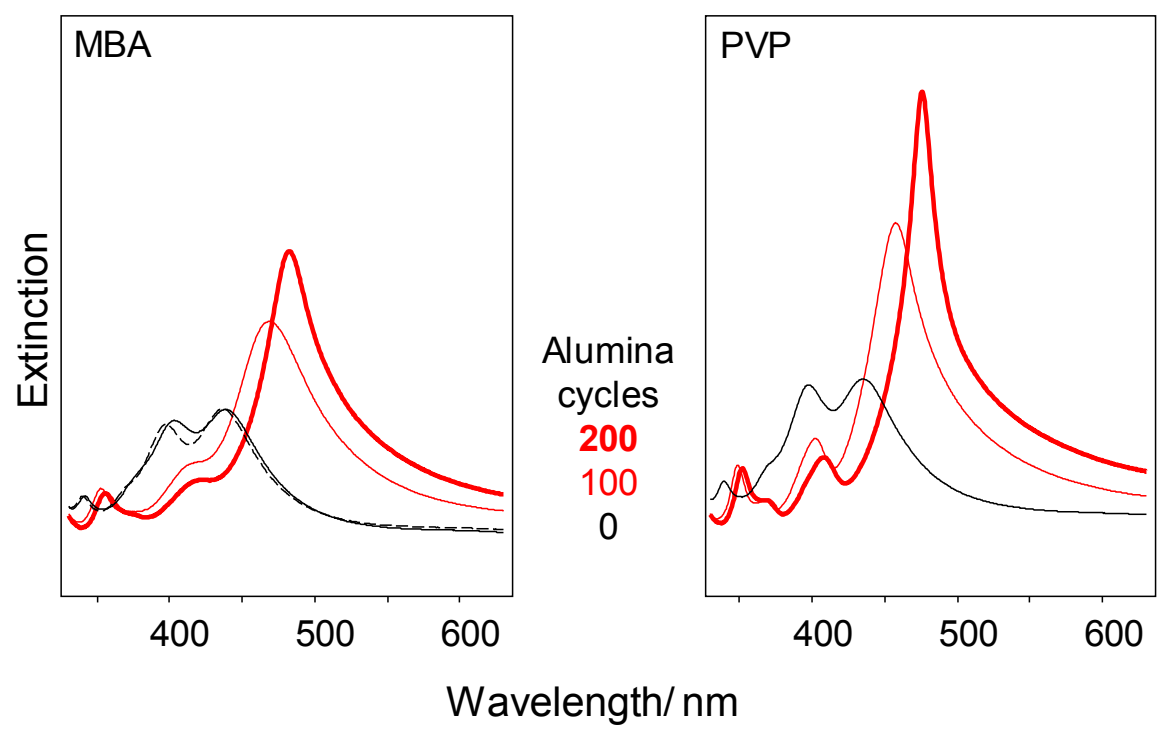

B.7 Uv-vis extinction spectra of supported silver nanocubes, subjected to ligand exchange with 4-mercaptobenzoic acid (MBA, left) and untreated polyvinylpyrrolidone (PVP, right) with zero (solid black line), 100 (thin red line), and 200 (thick red line) cycles of alumina ALD. Extinction spectrum of supported nanocubes before treatment with MBA is shown as a dashed line on the left. After the treatment with MBA (solid black line, left) the quadrupolar and dipolar peaks become spectrally closer, suggesting rounding of the cubes. Note: for this sample 200 cycles of alumina ALD were required to achieve the SLSPR conditions.
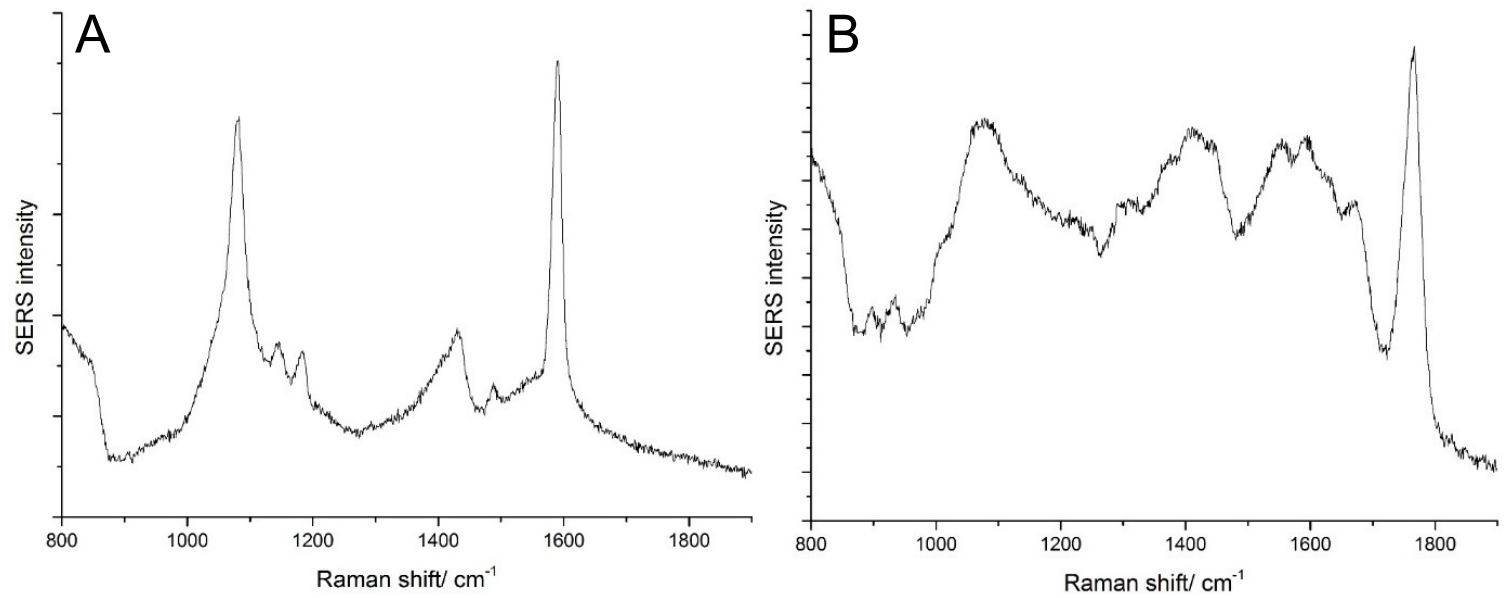

B.8 Representative SERS spectra of A) 4-mercaptobenzoic acid (MBA) and B) polyvinylpyrrolidone (PVP, right) 
A. $0 \mathrm{~nm} \mathrm{Al}{ }_{2} \mathrm{O}_{3}$

D: $438 \mathrm{~nm}$

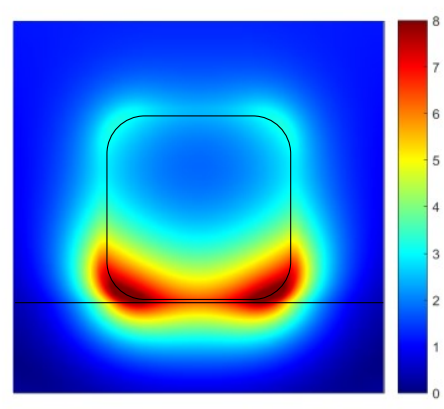

B. $5 \mathrm{~nm} \mathrm{Al}{ }_{2} \mathrm{O}_{3}$

D: $468 \mathrm{~nm}$

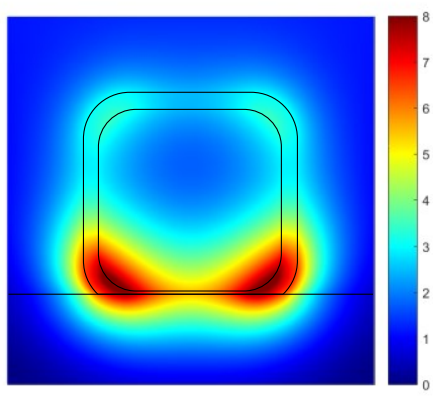

C. $10 \mathrm{~nm} \mathrm{Al} \mathrm{O}_{3}$

D: $492 \mathrm{~nm}$

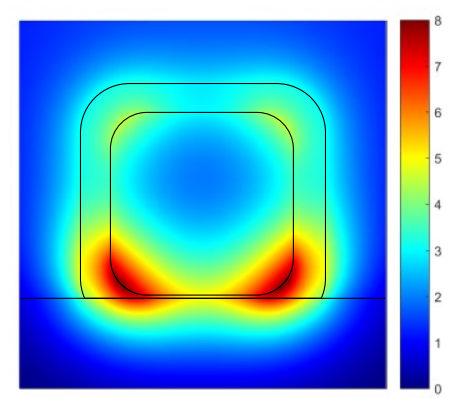

D. $12.5 \mathrm{~nm} \mathrm{Al}_{2} \mathrm{O}_{3}$

D: $502 \mathrm{~nm}$

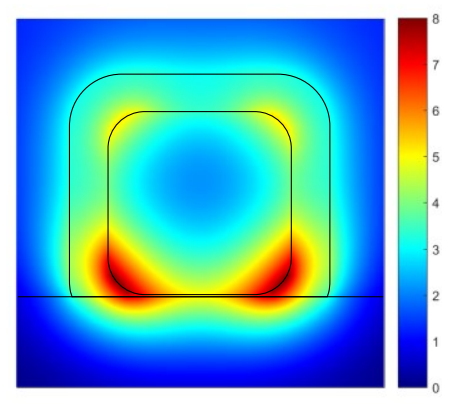

Q: $394 \mathrm{~nm}$
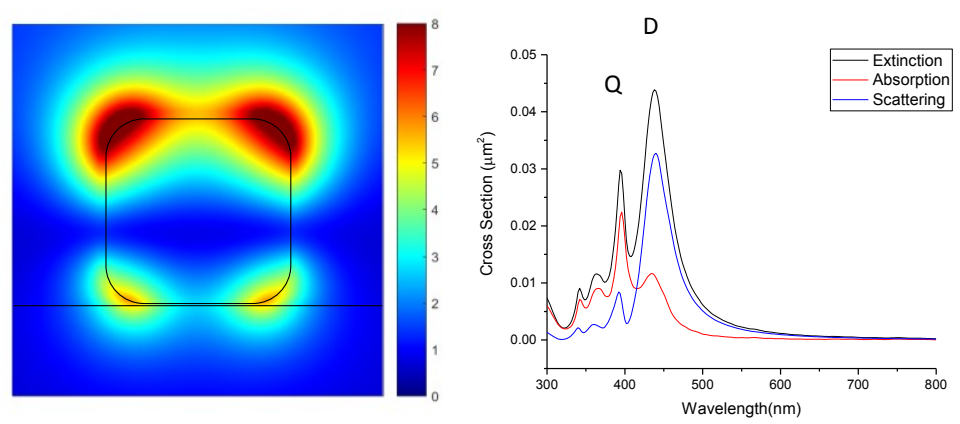

Q: $428 \mathrm{~nm}$

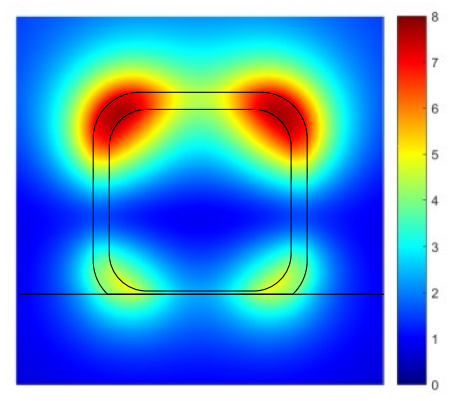

Q: $460 \mathrm{~nm}$

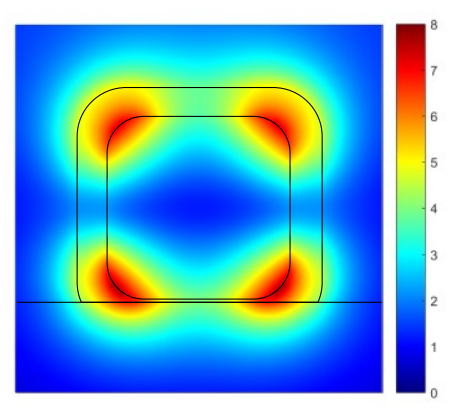

Q: $469 \mathrm{~nm}$

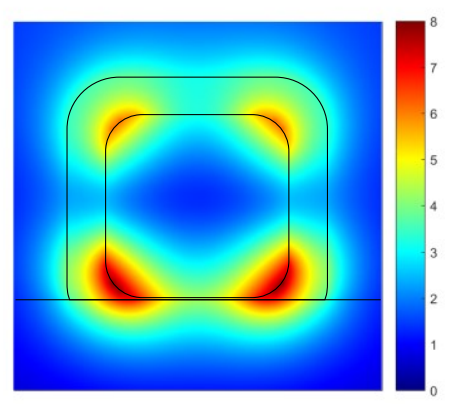

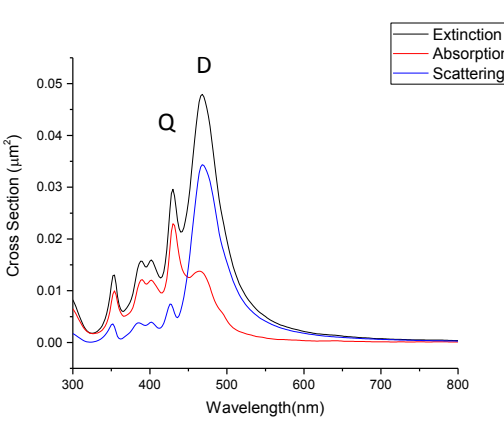
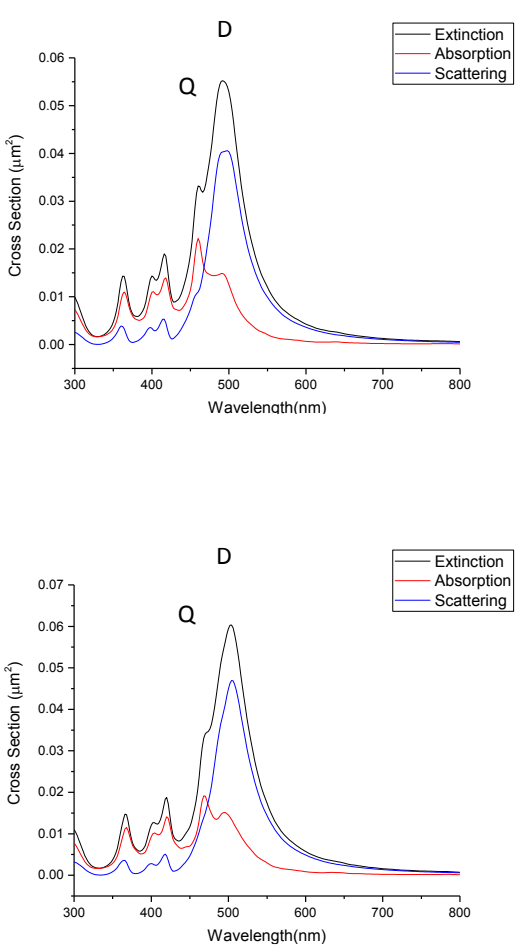


\section{E. $15 \mathrm{~nm} \mathrm{Al} \mathrm{O}_{3}$}

D: $510 \mathrm{~nm}$

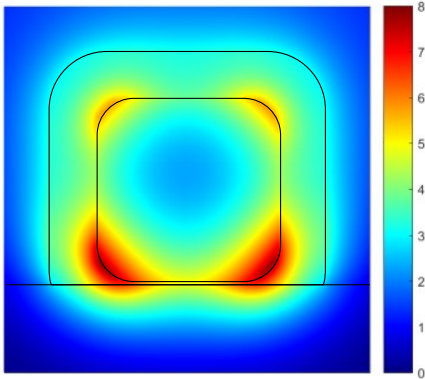

F. $20 \mathrm{~nm} \mathrm{Al} \mathrm{O}_{3}$

D: $522 \mathrm{~nm}$

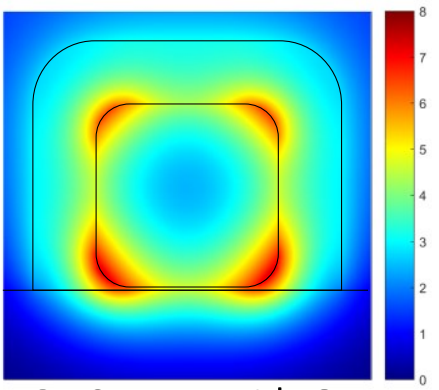

G. $25 \mathrm{~nm} \mathrm{Al}{ }_{2} \mathrm{O}_{3}$

D: $536 \mathrm{~nm}$

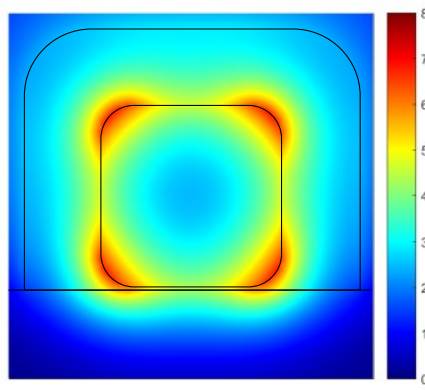

Q: $476 \mathrm{~nm}$
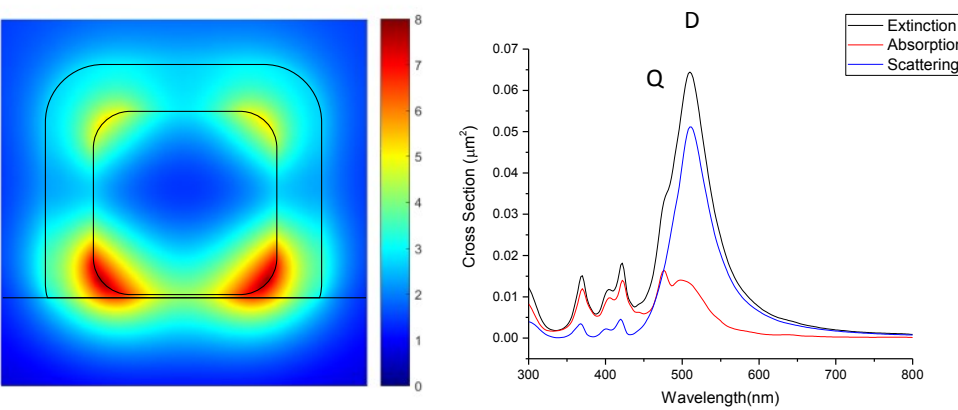

Q: $484 \mathrm{~nm}$
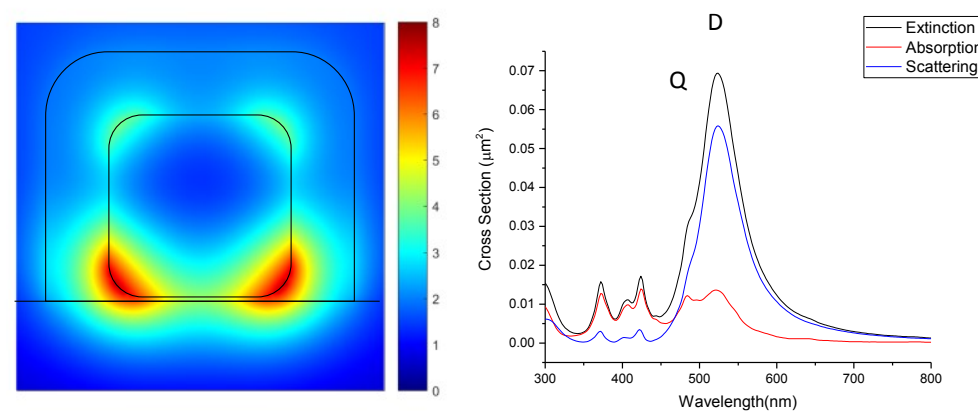

Q: $488 \mathrm{~nm}$
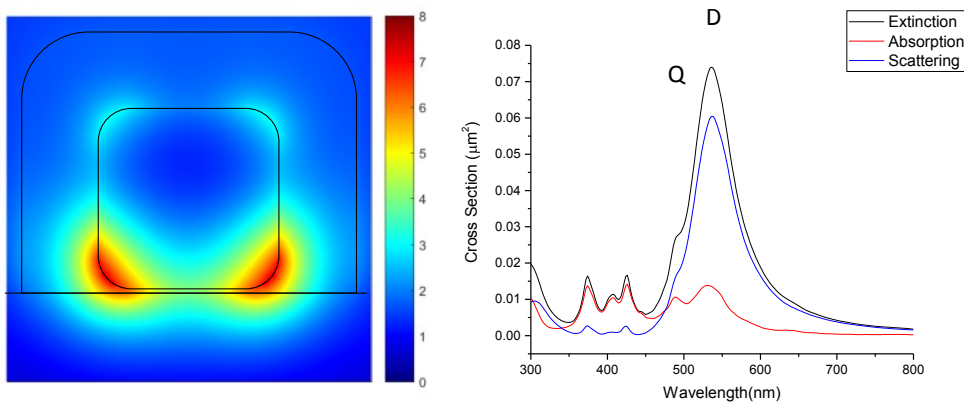

B.9 FDTD modelling of glass supported silver nanocube with a thin layer of alumina. Calculated electric field maps are presented for dipolar (D) and quadrupolar (Q) modes. Corresponding extinction spectra are also shown. Nanocube size is $60 \mathrm{~nm}$, corner roundness is $20 \%$ (12 $\mathrm{nm})$. Shell thickness is $0,5,10,12.5,15,20$ and $25 \mathrm{~nm}$ from top to bottom respectively. Rearrangement of charge spatial distribution for the quadrupolar mode is observed for shell thicknesses of 10 to $15 \mathrm{~nm}$. The mode converts from mostly distant from the substrate to proximal to the substrate. 


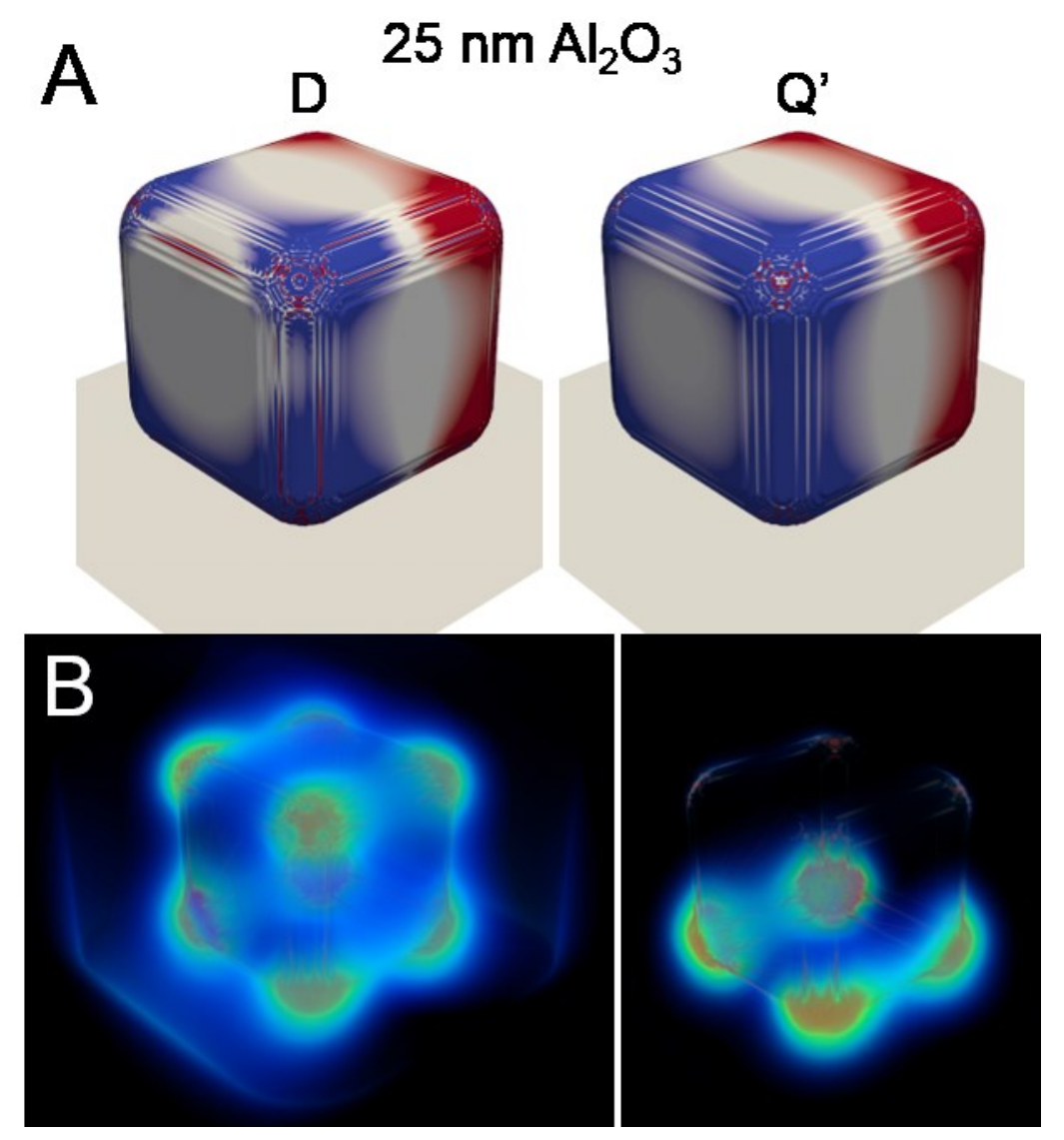

B.10 Calculated 3D divergence current maps (A) and $|E|^{2}$ field distribution (B) for glass supported silver nanocube with $25 \mathrm{~nm}(\mathrm{C})$ thick alumina shell. The hybrid quadrupolar mode (Q') becomes mostly dipolar in nature as the shell thickness exceeds $12.5 \mathrm{~nm}$, and becomes weakly located in the lower-index substrate at $25 \mathrm{~nm} \mathrm{Al}_{2} \mathrm{O}_{3}$. 


\section{Appendix C Supplementary Materials for Chapter 2C}
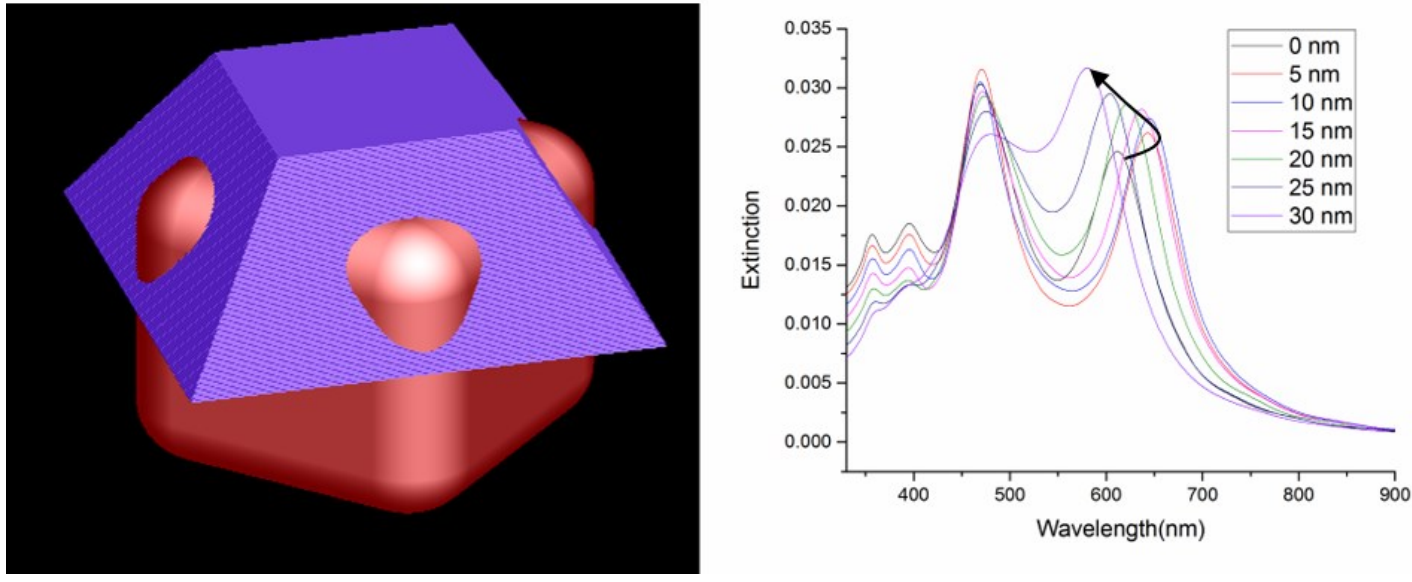

C.1 A schematic showing a pyramidal shaped hat when placed very low on the AgNC, causing the corners of the AgNC to poke out, right) this produced erratic results in the modelled spectra. Using larger $\mathrm{Cu}_{2} \mathrm{O}$ pyramids produced and excessive red-shift of the spectrum and was not suitable.
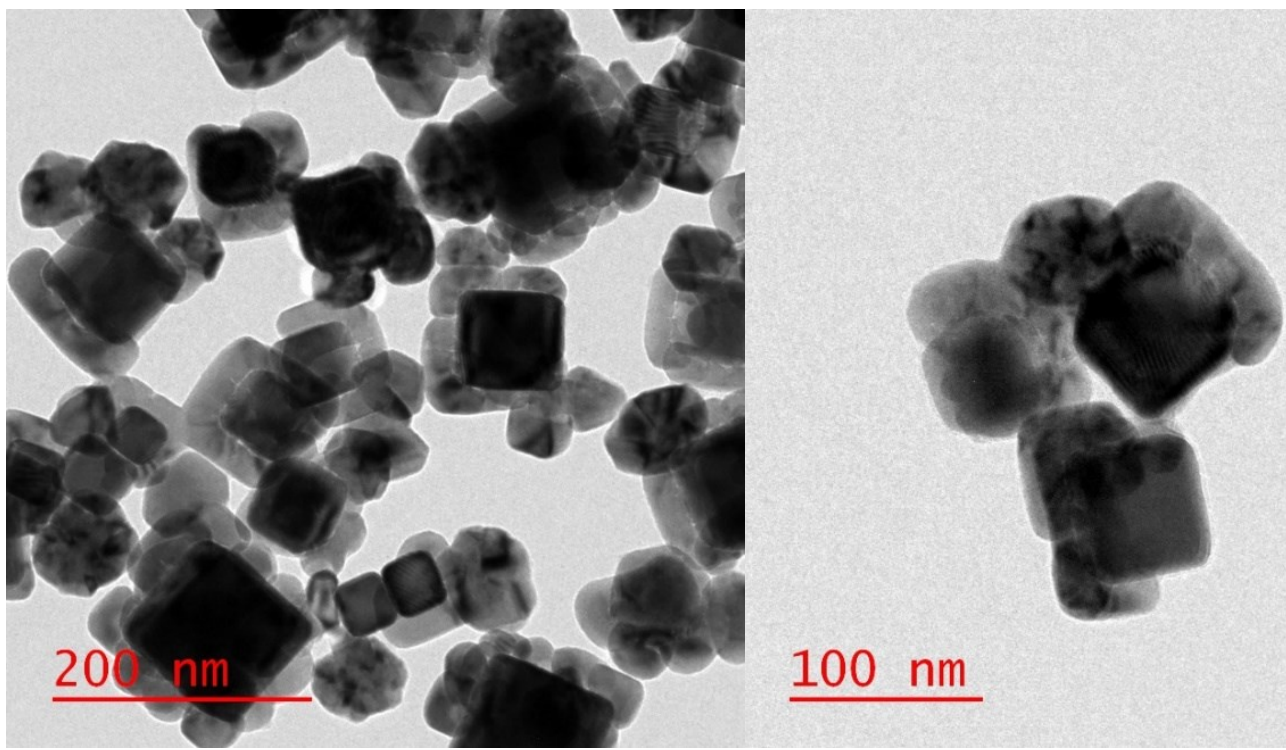

C.2 Experimentally produced $\mathrm{Ag} @ \mathrm{Cu}_{2} \mathrm{O}$ half-shells with more conformal, cubic-shaped shells. 

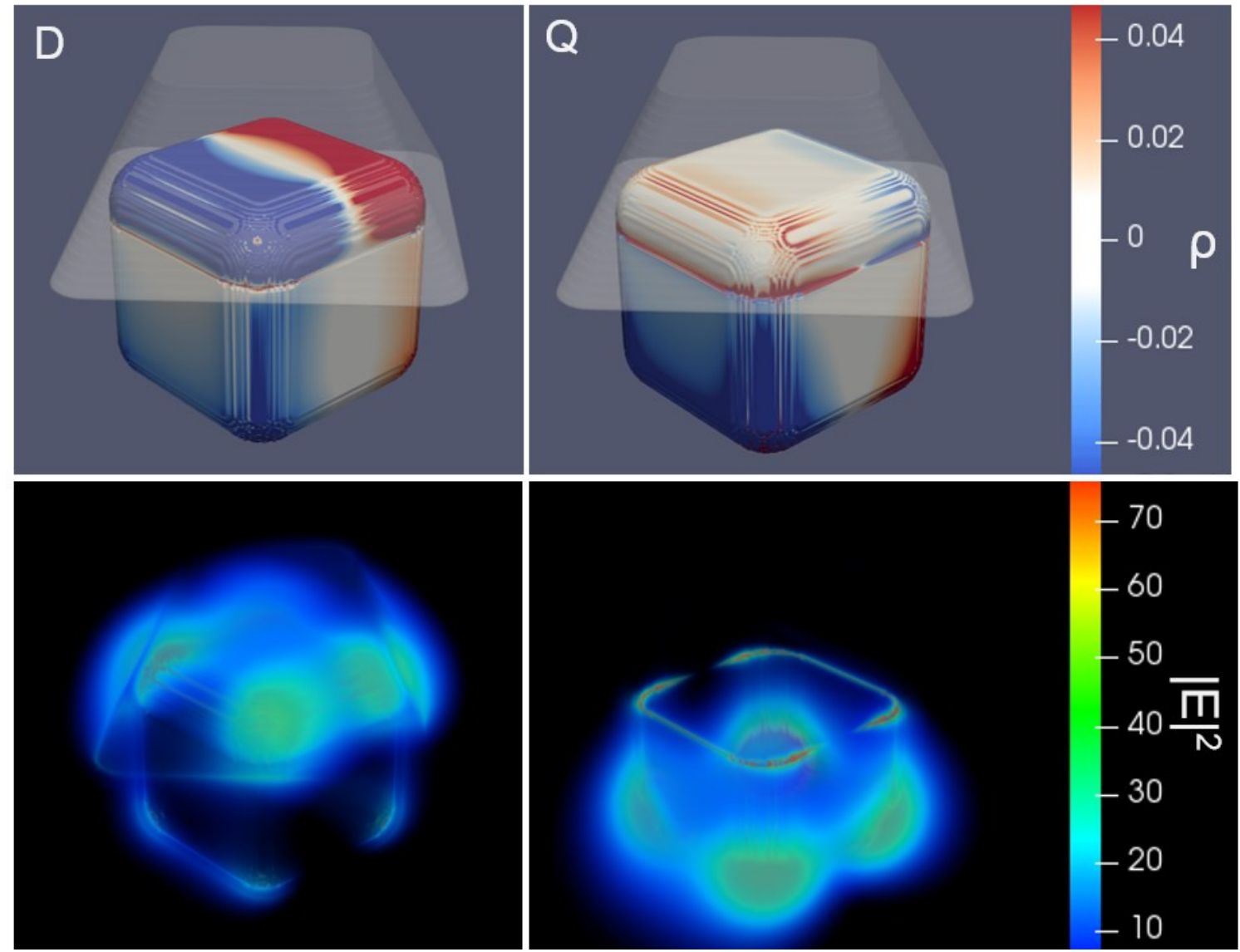

C.3 Top) 3D charge density ( $\rho$ ) and bottom) $|E|^{2}$ enhancement of pyramidal $\mathrm{Cu}_{2} \mathrm{O}$ half-shells, displaying the dipolar and quadrupolar modes. 


\section{Appendix D Supplementary Materials for Chapter 3}
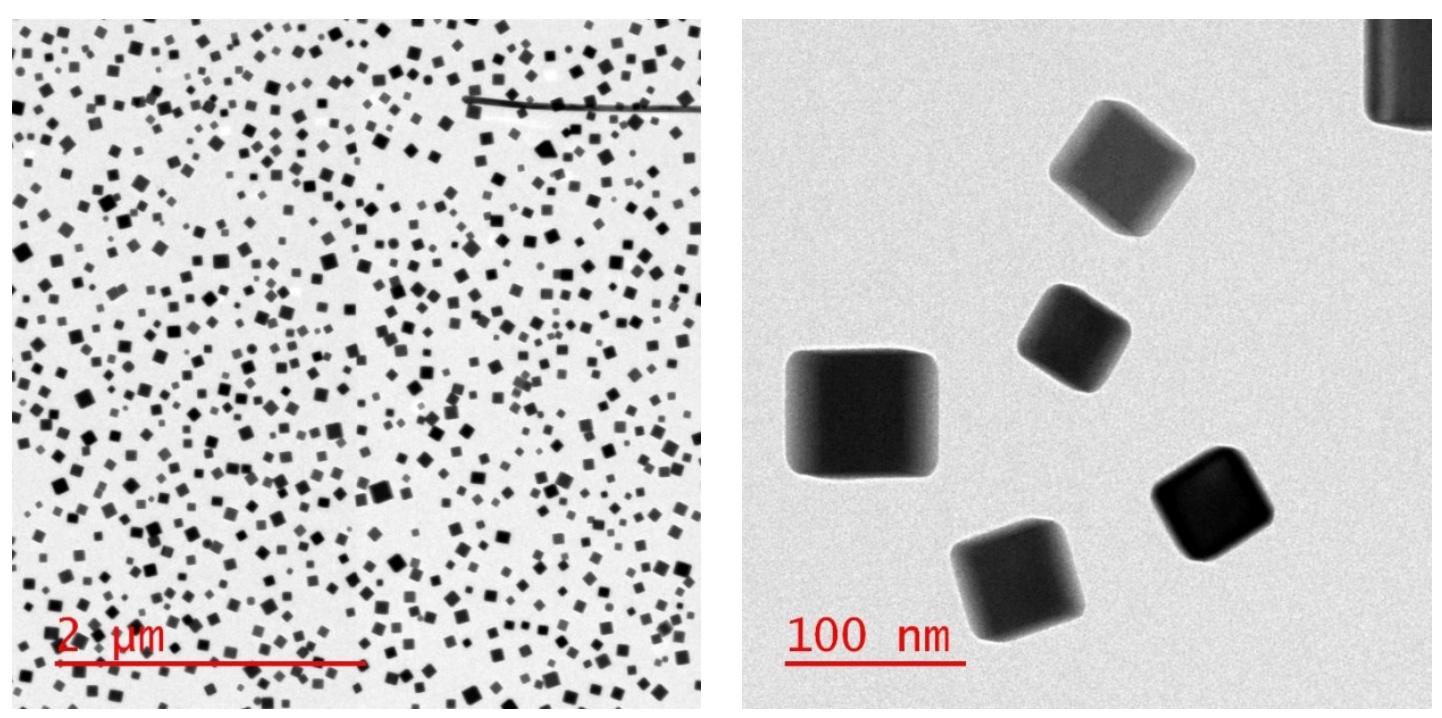

D.1 Transmission electron micrographs of as-synthesized silver nanocubes.

Source: $457.9 \mathrm{~nm}$

time (s)

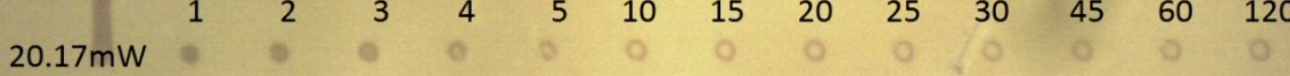

$15.08 \mathrm{~mW}$

$10.20 \mathrm{~mW}$

D.2 Sequence of patterning performed at $458 \mathrm{~nm}$ using three different incident powers (10, 15, $20 \mathrm{~mW}$ ), with exposure times varying from $1-120 \mathrm{~s}$. Each power produced a consistent spot size, though the area of decomposed polymer increased at 15, and $20 \mathrm{~mW}$. Below 10 $\mathrm{mW}$, no changes were observed, regardless of exposure time. 

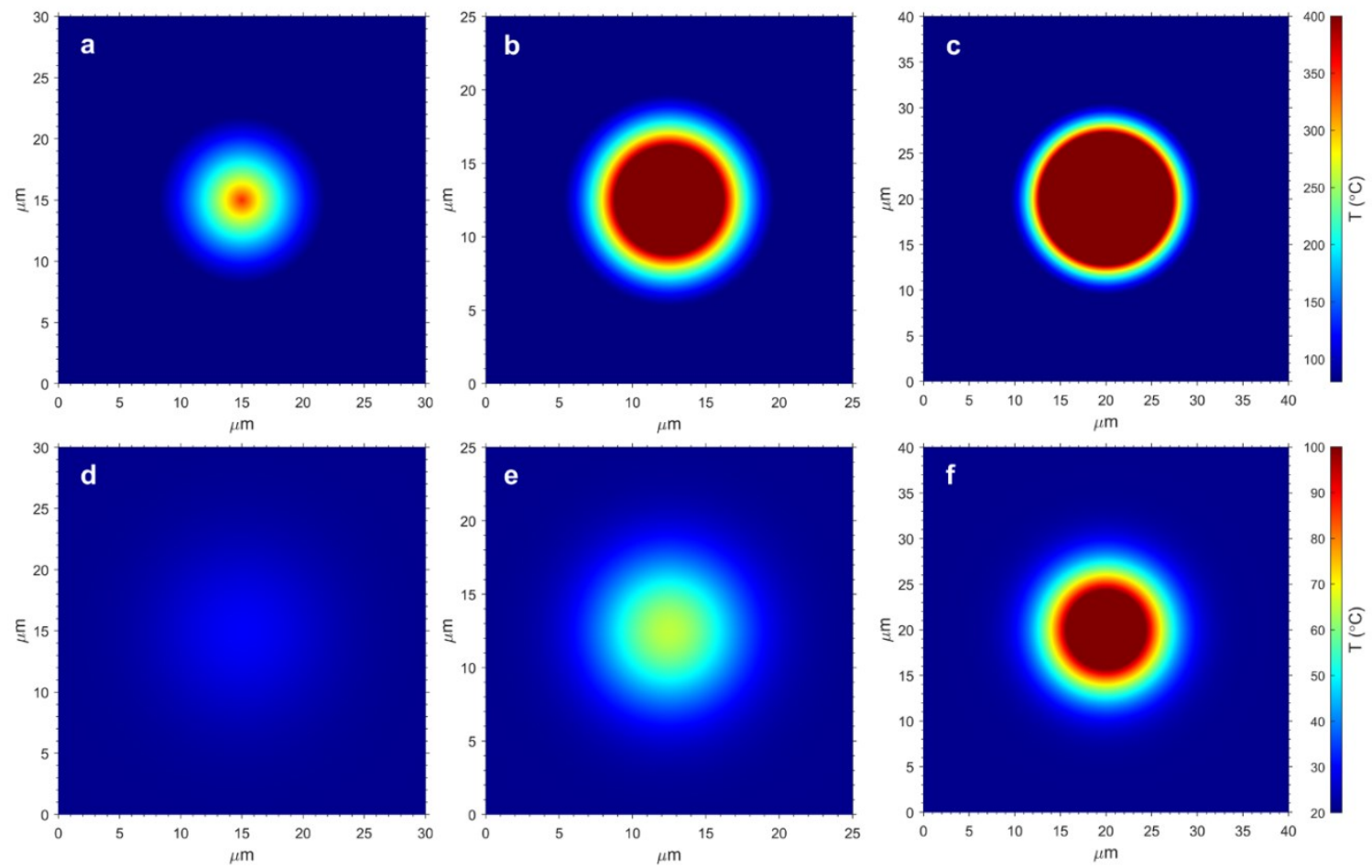

D.3 Calculated temperature maps including the contribution for collective heating using equation (3) $(a, b, c)$ and for individual heating using equation (1) (d,e,f). Note the difference in z-scale (color bar). For the conditions in a, d $(75 \mathrm{~mW}, 568 \mathrm{~nm})$ the particles individual contribution to heating produces a hardly noticeable change. In state II (b,e) (20 mW. $476 \mathrm{~nm}$ shown), the average temperature is 100's degrees lower and still likely incapable of producing a change in the polymer film. In state III (c,f) $(50 \mathrm{~mW}, 458 \mathrm{~nm}$ shown) the maximum temperature is predicted to reach $155^{\circ} \mathrm{C}$, still insufficient for decomposition of the polymer. This demonstrates the necessity for collective heating among the nanocubes to explain the observed physical changes in the polymer.
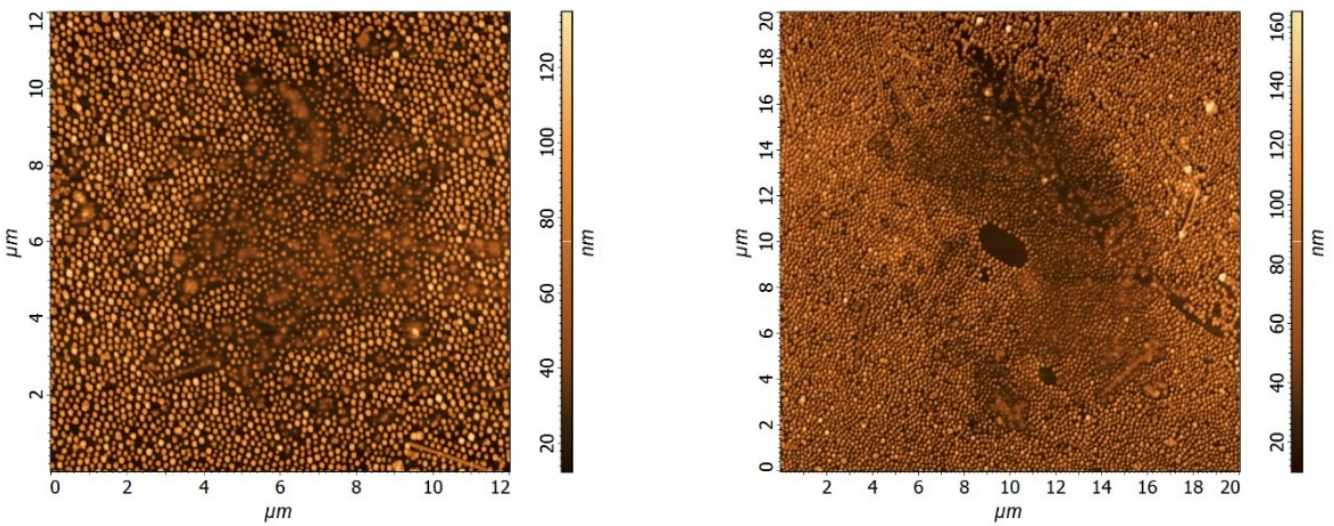

D.4 Atomic force micrographs of patterned areas under $647 \mathrm{~nm}$ excitation at high power (left, $100 \mathrm{~mW}$, right, $150 \mathrm{~mW}$ ). Embedment of nanocubes is achieved but the arrangement is sporadic and does not follow the gaussian profile of the beam. 
D.5 Waist diameter determinations and conversions to peak intensity of the incident beam. Peak intensity being the maximum intensity of the gaussian-profile beam, defined as $I=\frac{2 P}{\pi \omega_{0}{ }^{2}}$.

\begin{tabular}{llllll} 
Wavelength & $458 \mathrm{~nm}$ & $476 \mathrm{~nm}$ & $514 \mathrm{~nm}$ & $568 \mathrm{~nm}$ & $647 \mathrm{~nm}$ \\
\hline Waist radius $(\mu \mathrm{m})$ & $8.5 \pm 0.3$ & $7.3 \pm 0.3$ & $7.6 \pm 0.4$ & $10.2 \pm 1.0$ & $10.9 \pm 0.7$ \\
Power $(\mathrm{mW})$ & Intensity & $\left(\mathrm{mW} / \mu \mathrm{m}^{2}\right)$ & & & \\
\hline 5 & 0.043 & 0.060 & 0.056 & 0.031 & 0.027 \\
10 & 0.087 & 0.12 & 0.11 & 0.062 & 0.054 \\
15 & 0.13 & 0.18 & 0.17 & 0.092 & 0.081 \\
20 & 0.17 & 0.24 & 0.22 & 0.12 & 0.11 \\
25 & 0.22 & 0.30 & 0.28 & 0.15 & 0.13 \\
50 & 0.35 & 0.48 & 0.44 & 0.25 & 0.22 \\
75 & 0.39 & 0.54 & 0.50 & 0.28 & 0.24 \\
100 & 0.43 & 0.60 & 0.56 & 0.31 & 0.27 \\
125 & 0.65 & 0.90 & 0.83 & 0.46 & 0.40 \\
150 & 0.87 & 1.20 & 1.11 & 0.62 & 0.54
\end{tabular}




\section{Appendix E Supplementary Materials for Chapter 4}
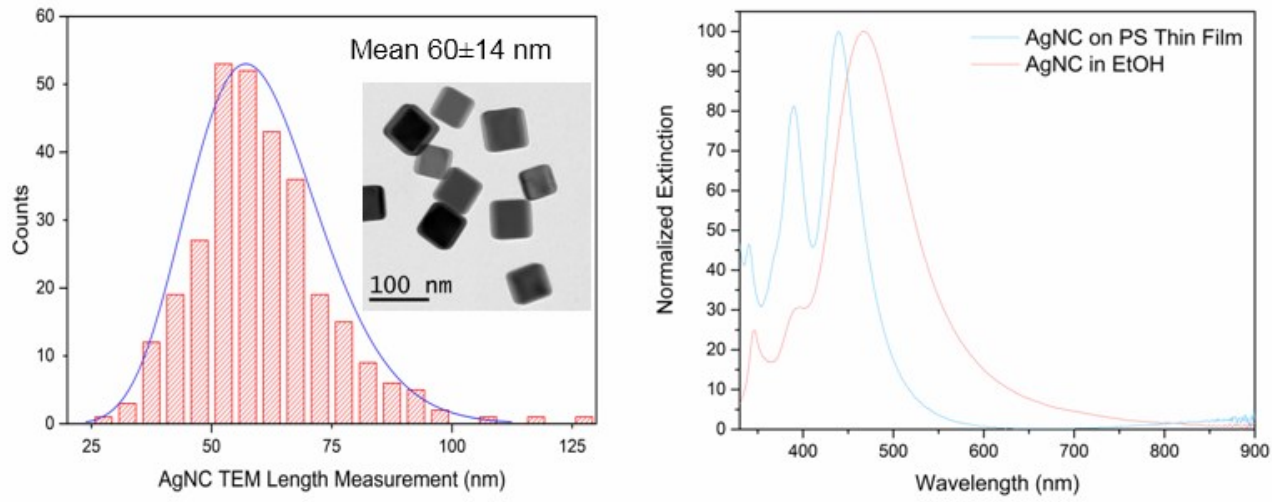

E.1 Size distribution from transmission electron microscopy, as well as UV-Vis extinction of assynthesized $\mathrm{AgNC}$, as well as $\mathrm{AgNC}$ deposited onto polystyrene over $\mathrm{SiO}_{2}$ with no metal film component.
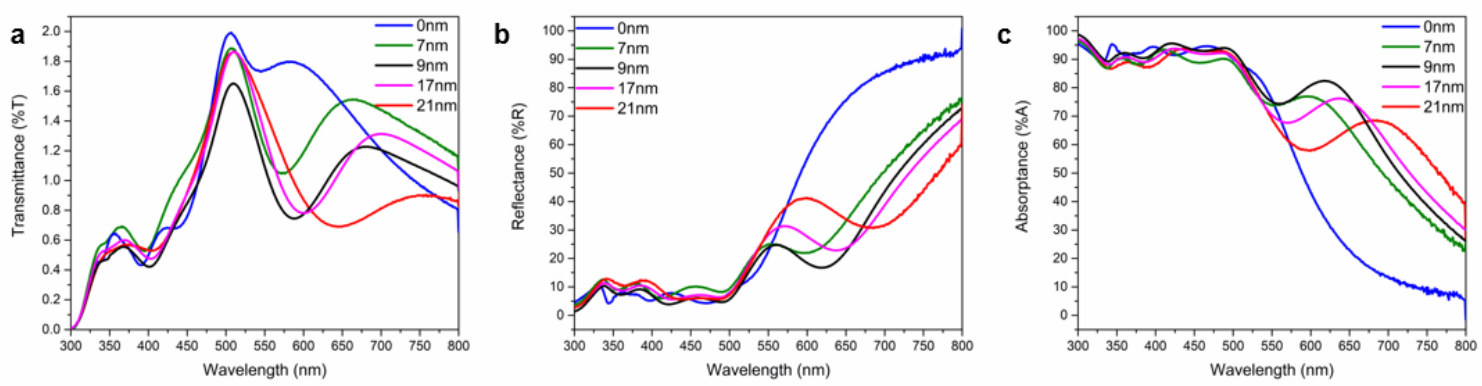

E.2 a) Transmittance, b) Reflectance, and c) calculated Absorptance, where $A=100-(T+R)$. The transmittance of the sample is weak, thus the spectral response is primarily attributed to absorbance. 

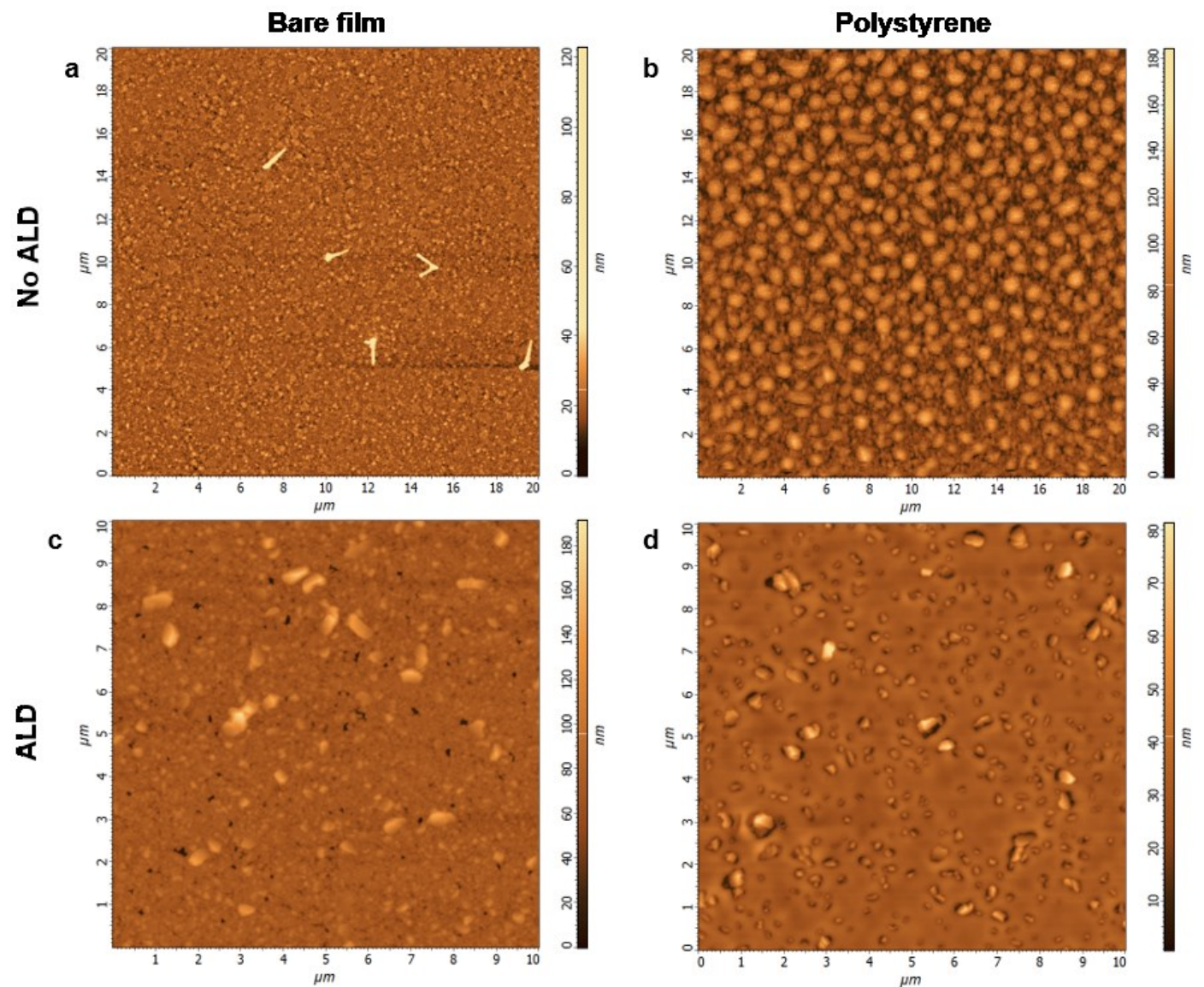

E.3 AFM of Ag films with (a) and without (b) ALD of $\mathrm{Al}_{2} \mathrm{O}_{3}$, and result of respective annealed polystyrene thin films (b, d). Without $\mathrm{Al}_{2} \mathrm{O}_{3}$, annealing of the PS film resulted in island formation (d). With ALD smoother films were achieved (d), however the initial silver film was roughened (c)

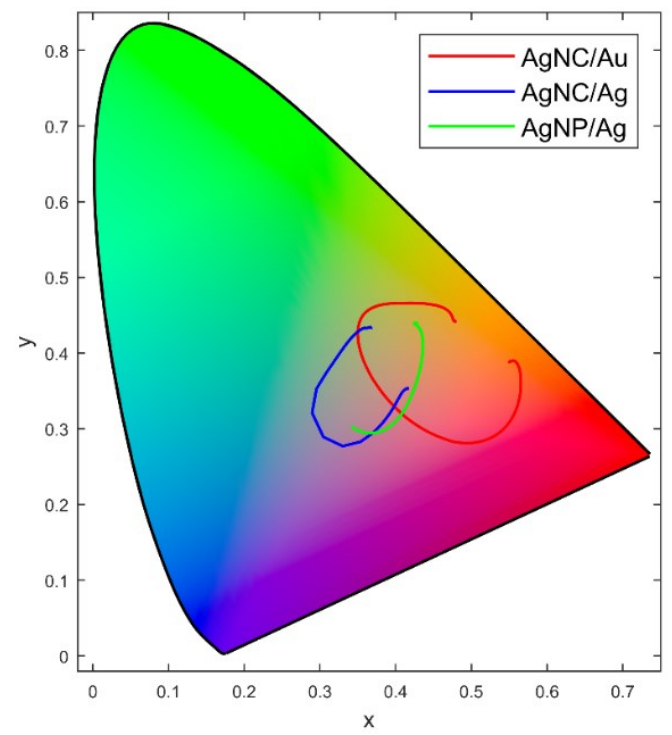

E.4 Chromaticity plots comparing three the AgNC over Au and Ag films, as well as spherical AgNP over Ag films 
a

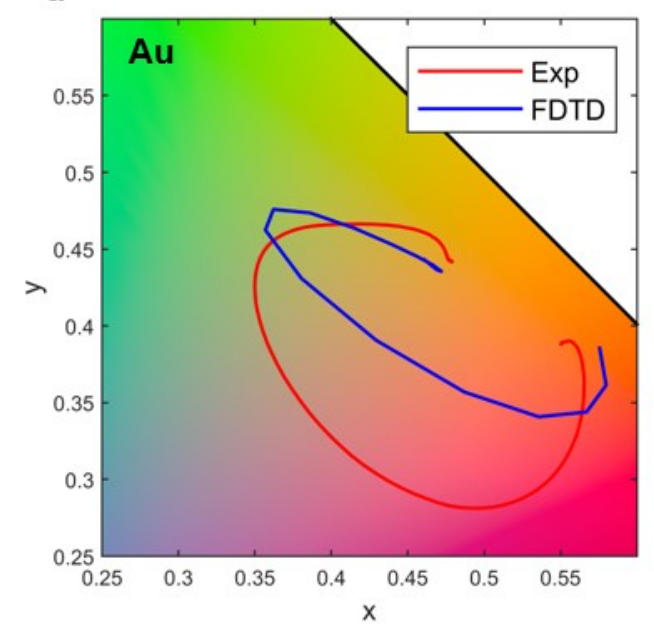

b

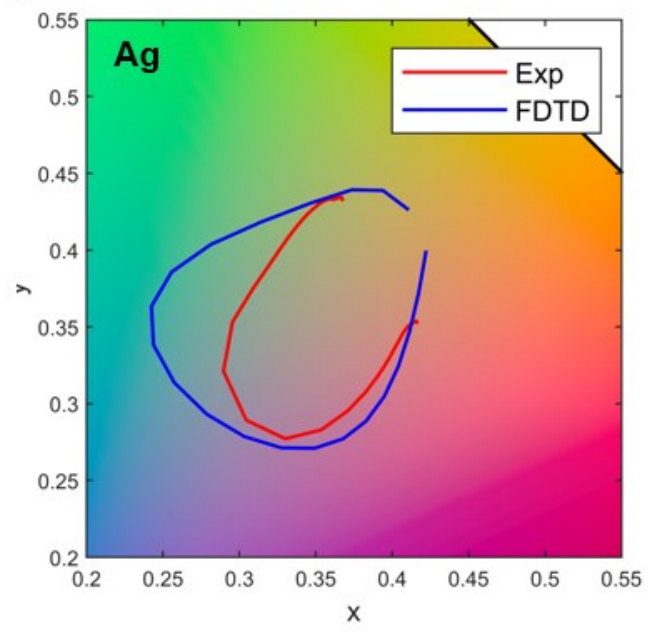

E.5 Chromaticity plots comparing the experimental and modelled results for a) $\mathrm{Au}$ and b) $\mathrm{Ag}$ films.

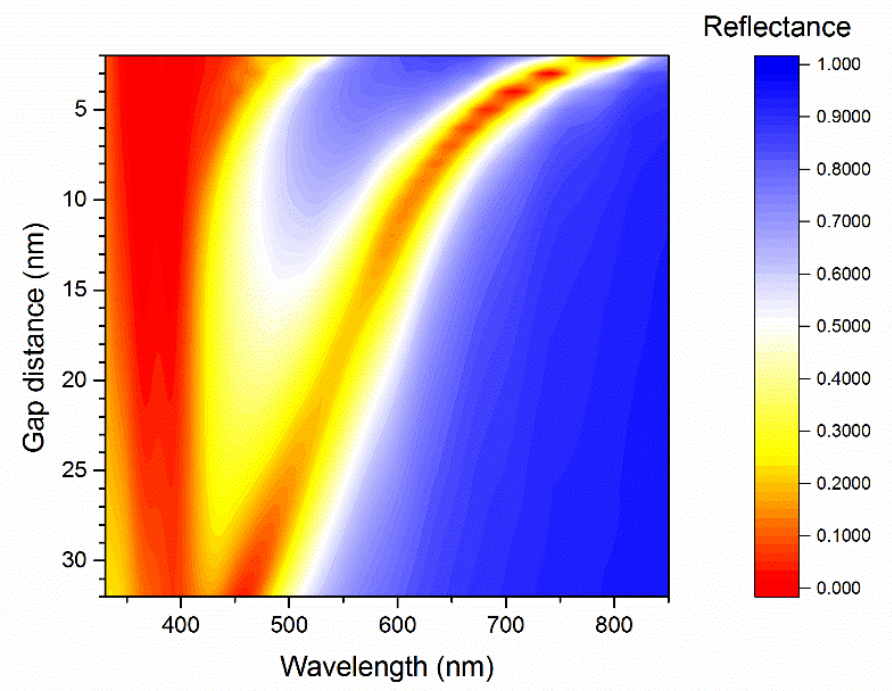

E.6 Spectral colormap predicting the results of an AgNC/Ag system with an initial PS thickness of $30 \mathrm{~nm}$. The initial plasmon position is at a lower wavelength of $\sim 455 \mathrm{~nm}$. 


\section{Appendix F Supplementary Materials for Chapter 5}

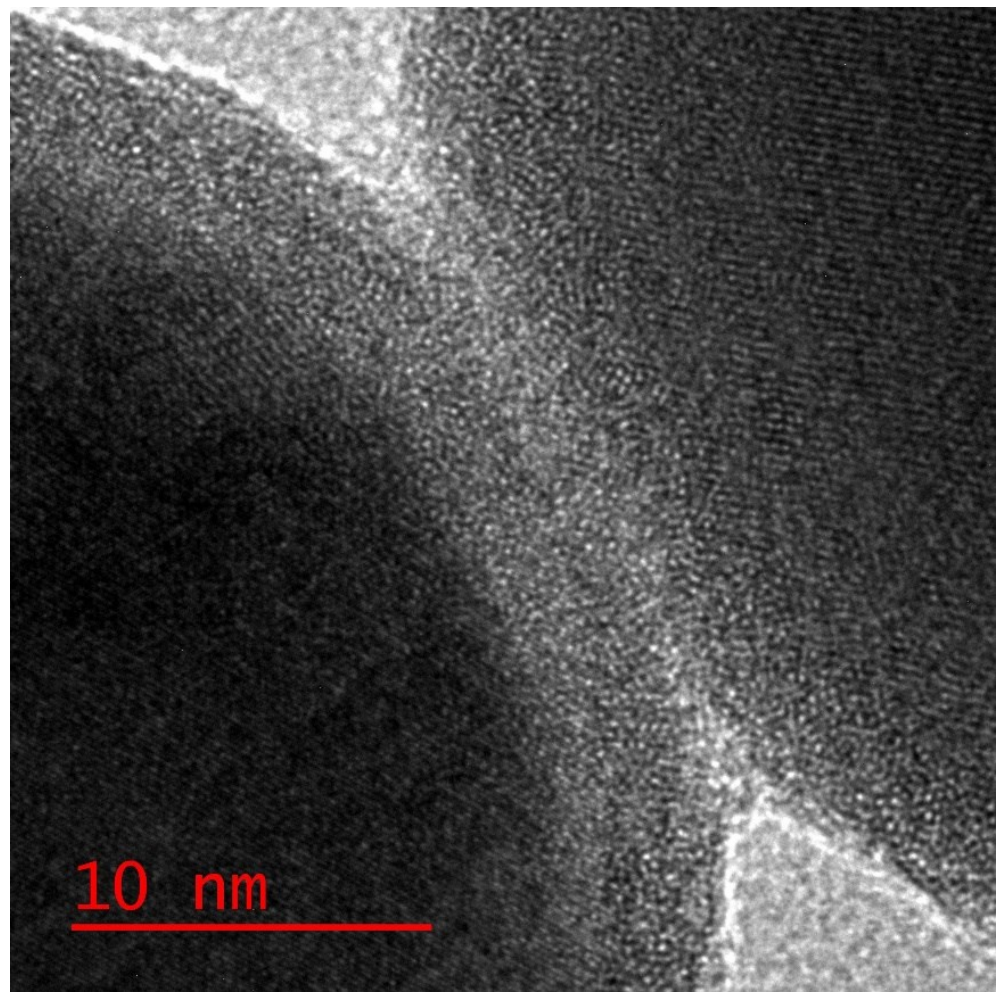

F.1 Transmission electron micrograph depicting the native $\ln _{2} \mathrm{O}_{3}$ oxide shell at a junction of indium nanoparticles.

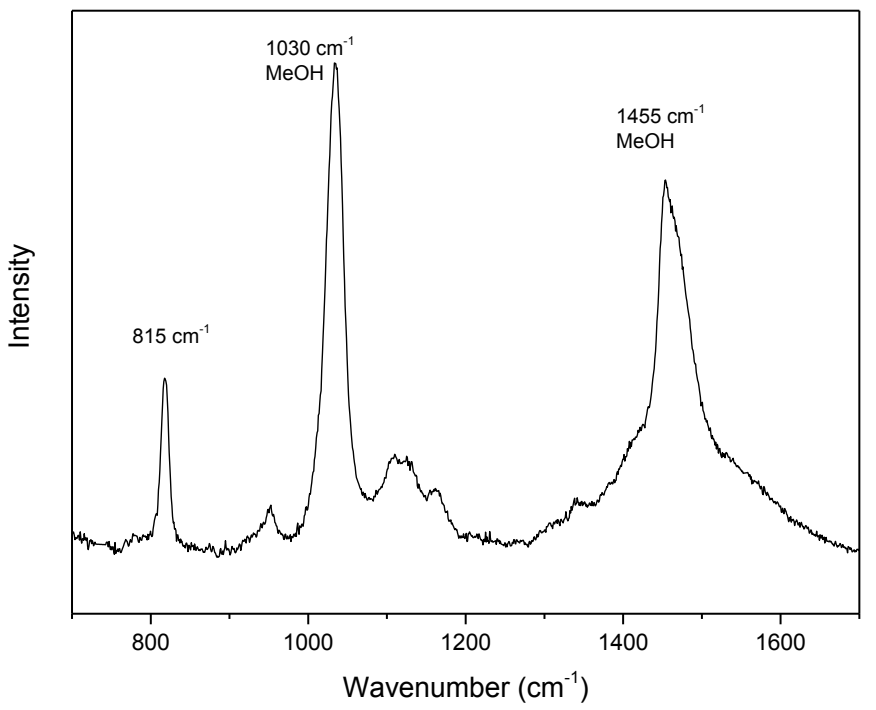

F.2 UV Raman spectrum of In@SiO 2 particles in $\mathrm{MeOH}$. A new mode at $815 \mathrm{~cm}^{-1}$ appears which is attributed to either $\mathrm{SiO}_{4}$ or SiO-C stretches; likely remnants from the incomplete hydrolysis of TEOS. 


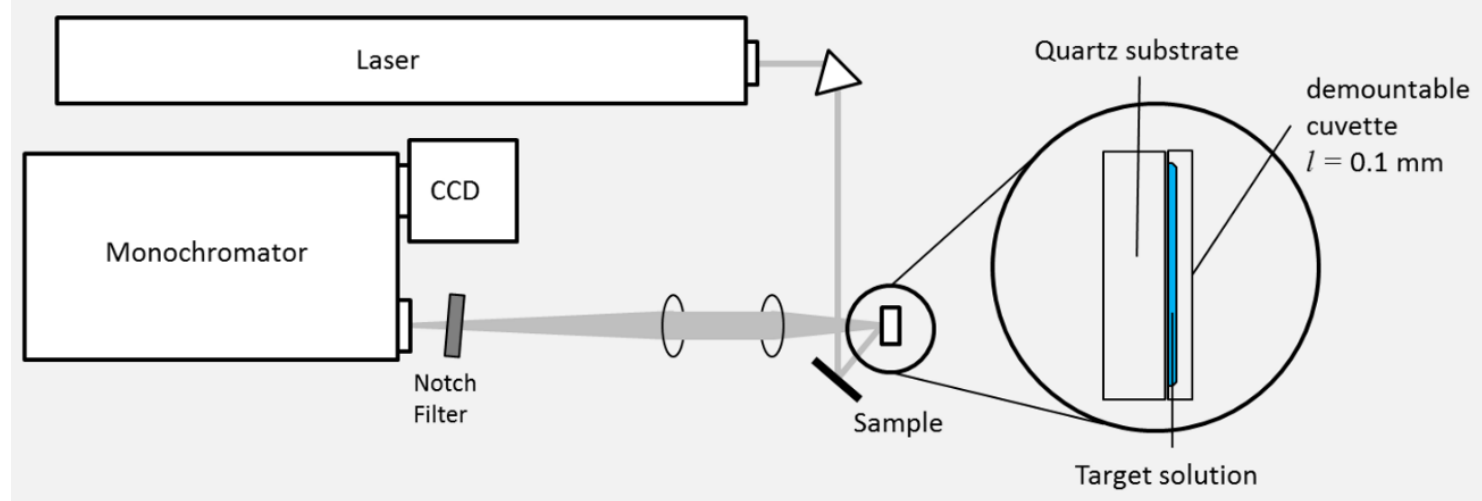

F.3 Schematic diagram of the UV-Raman setup when using a demountable cuvette. InNP or AgNP layers are cast onto a quartz substrate, and a thin $(0.1 \mathrm{~mm})$ analyte solution is suspended above the sample.

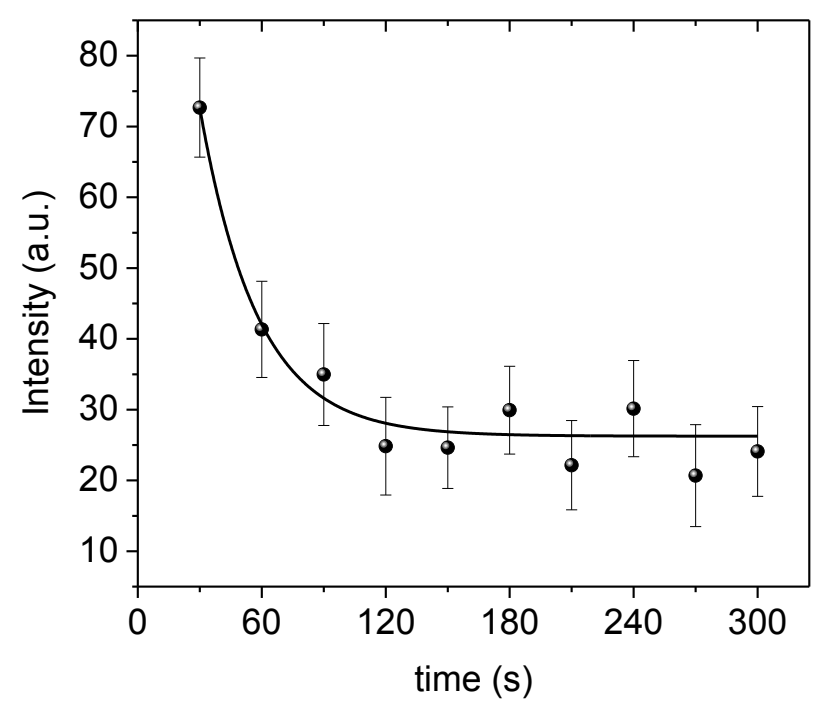

F.4 Kinetics of UV Raman of a $1 \mathrm{mM}$ Ala-Phe solution. The intensity of the $1004 \mathrm{~cm}^{-1}$ peak decreases with exposure time, reaching a minimum at $\sim 120 \mathrm{~s}$. 

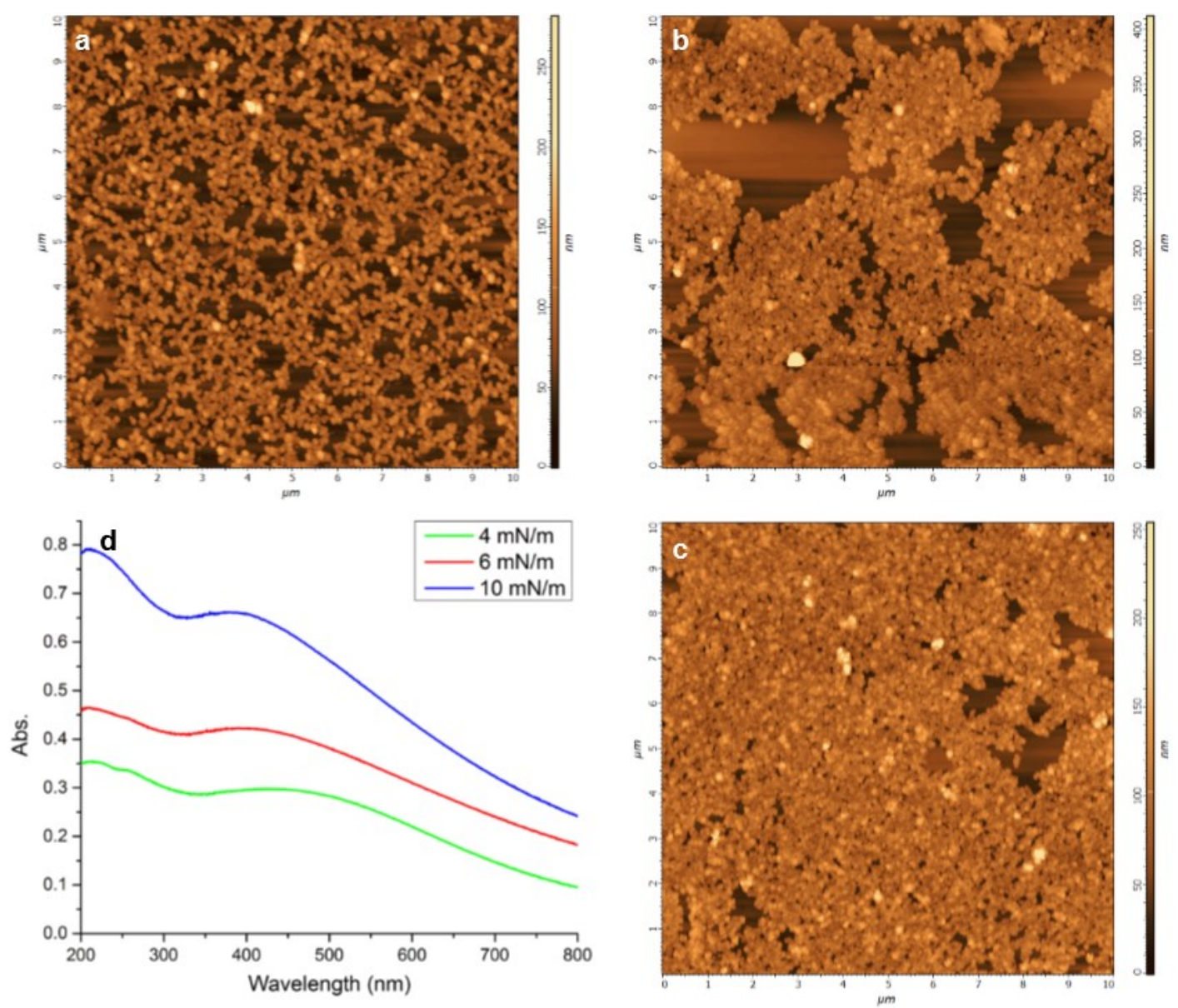

F.5 $10 \times 10 \mu \mathrm{m}$ AFM topography of LB films of InNP deposted at a) $4 \mathrm{mN} / \mathrm{m}$, b) $6 \mathrm{mN} / \mathrm{m}$, and 10 $\mathrm{mN} / \mathrm{m}$, as well as d) associated UV-Vis extinction

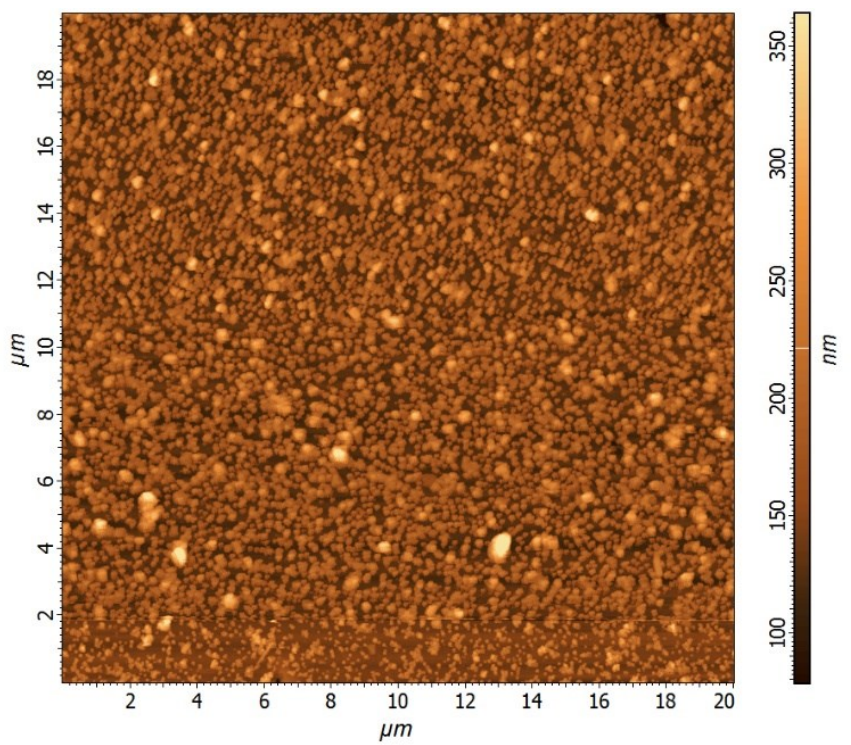

F.6 AFM topgraphy of AINP's spincoated onto a quartz substrate, used in UV-SERS experiments. 


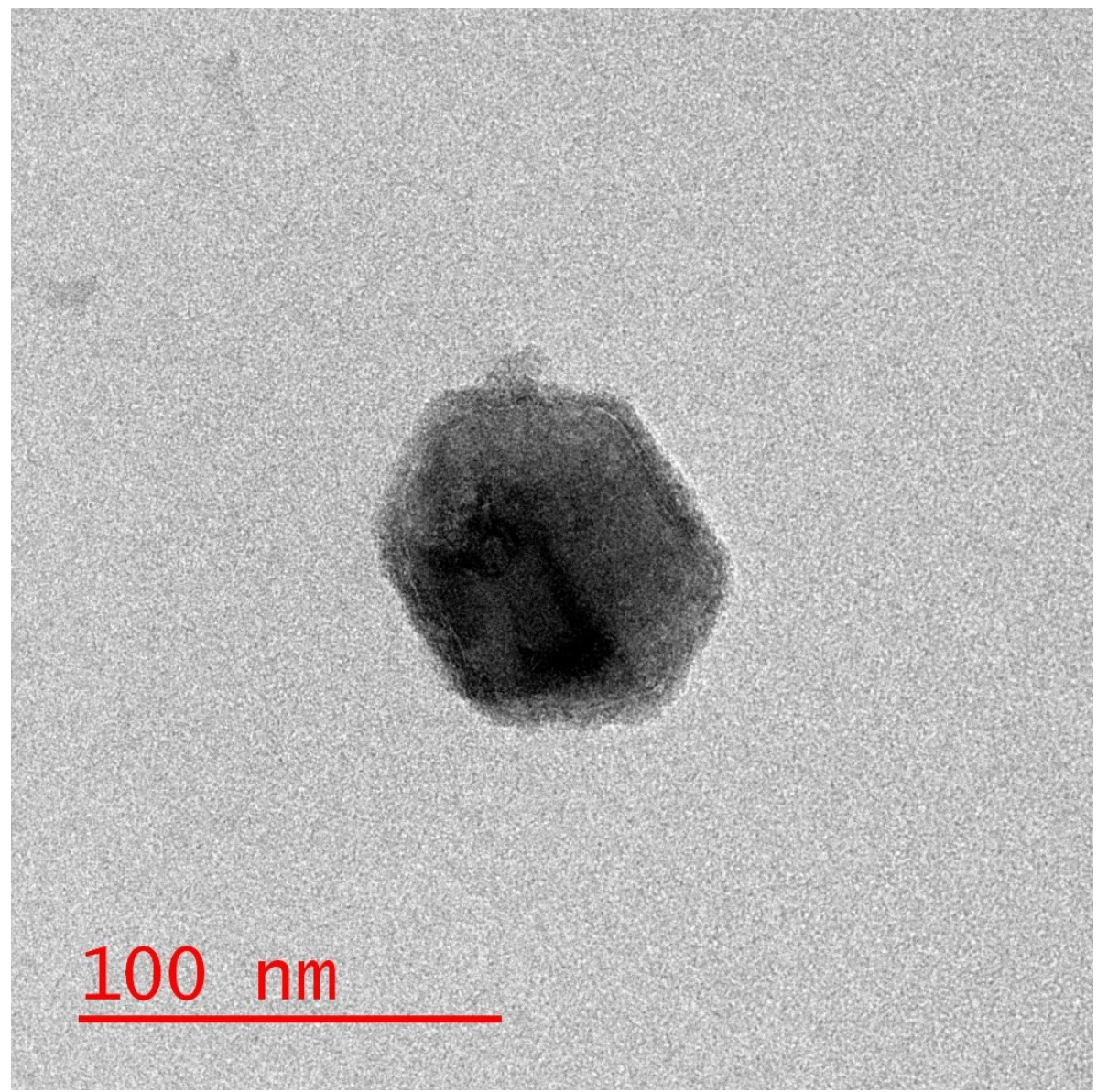

F.7 Transmission electron micrograph depicting an AINP thinly over-coated with $\mathrm{SiO}_{2}$ 


\section{Appendix G List of Contributions}

This section includes all contributions towards scientific publishing and presentations made during the attainment of this degree, including those not presented in this work.

\section{Peer-Reviewed Publications}

Bottomley, A.; Prezgot, D.; Coyle, J. P.; lanoul, A. Dynamics of Nanocubes Embedding into Polymer Films Investigated via Spatially Resolved Plasmon Modes. Nanoscale 2016, 8 (21), 11168-11176.

Prezgot, D.; Bottomley, A.; Coyle, J. P.; lanoul, A. Unusually Sharp Localized Surface Plasmon Resonance in Supported Silver Nanocrystals with a Thin Dielectric Coating. J. Phys. Chem. Lett. 2017, 8 (22), 5555-5558.

Gale-Mouldey, A.; Jorgenson, E.; Coyle J. P.; Prezgot, D.; lanoul, A.; Hybridized plasmon resonances in core/half-shell cuprous oxide nanoparticles., J. Mater Chem. C., 2020, Advance Article

D. ONeill, D. Prezgot, A. Ianoul, C. Otto, G. Mul, A. Huijser., Silver Nanocubes Coated in Ceria: Core/Shell Size Effects on Light-induced Charge Transfer, ACS Appl. Mater. Interfaces, 2020, 12, 1905-1912

\section{Manuscripts in preparation}

Prezgot, D.; Bottomley, A.; Jorgenson, E.; lanoul, A. Thermoplasmonic patterning of silver nanocrystal/polymer composite thin films, Manuscript in preparation

Prezgot, D.; Tatarchuk, S.W.; lanoul, A. Plasmonic Colour Generation in Silver Nanocrystal-overMirror Films by Thermal Embedment into a Polymer Spacer, Manuscript in Preparation

\section{Conference Presentations}

Prezgot, D..; Ianoul, A. Deep-Ultraviolet Surface-Enhanced Raman Spectroscopy of Biomolecules on Indium Nanoparticles, $98^{\text {th }}$ Canadian Chemistry Conference and Exhibition, Ottawa, ON, Canada, Jun 2015, Oral Presentation

Prezgot, D.; Bottomley, A.; Coyle, J. P.; lanoul, A.. Connecting Far-Field Optical Repsonse and SERS in Unusually Sharp Localized Surface Plasmon Resonances Produced in Silver Nanocrystals, SERS - Faraday Discussion, Glasgow, United Kingdom, Sep 2017 Poster Presentation

Prezgot, D.; Bottomley, A.; Jorgenson, E.; lanoul, A. Thermoplasmonic Patterning in Silver Nanocrystal/Polymer Thin Film Composites, Nano 2018, Hong Kong, HK SAR, China, Jun 2018, Oral Presentation

Prezgot, D.; Bottomley, A.; Jorgenson, E.; lanoul, A. Thermoplasmonic Patterning in Silver Nanocrystal/Polymer Thin Film Composites, Nano Ontario, Ottawa, ON, Canada, Nov 2018, Oral Presentation

Prezgot, D.; Bottomley, A.; Jorgenson, E.; lanoul, A. Thermoplasmonic Patterning in Silver Nanocrystal/Polymer Thin Film Composites, $100^{\text {th }}$ Canadien Chemistry Conference and Exhibition, Toronto, ON, Canada, Jun 2017

Prezgot, D.; Tatarchuck, S.W.; lanoul, A. Plasmonic Colour Generation in Silver by thermal embedment of silver nanocrystals into supported polymer films. 102 ${ }^{\text {nd }}$ Canadian Chemistry Conference and Exhibition, Quebec, QC, Canada, Jun 2019, Poster Presentation 